


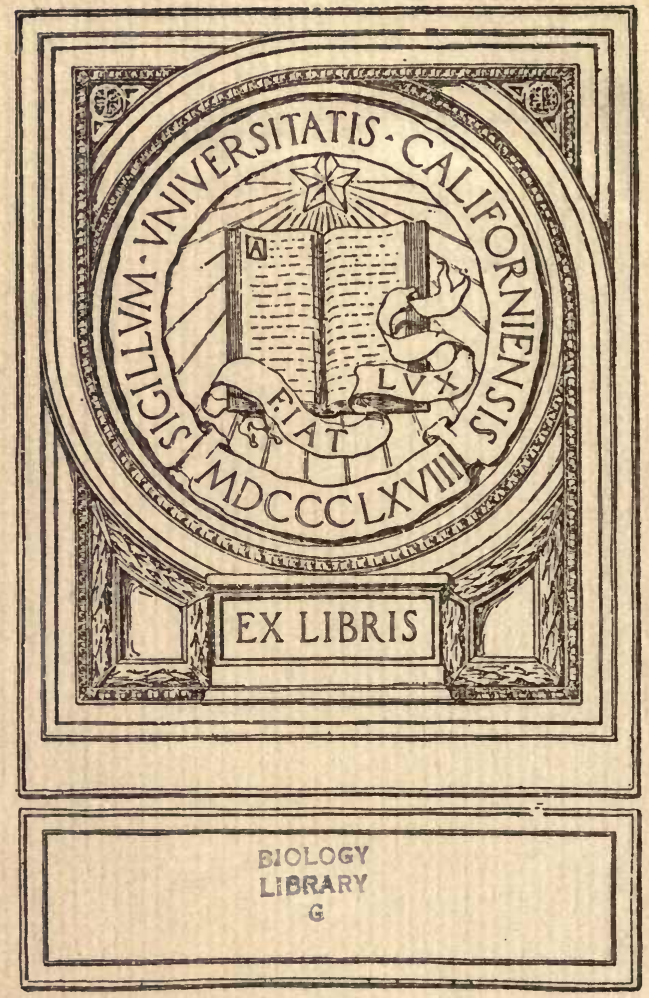





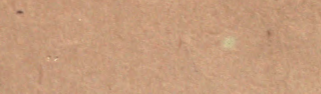




AN INTRODUCTION TO GENERAL PHYSIOLOGY 



\section{AN INTRODUCTION TO}

\section{GENERAL PHYSIOLOGY.}

\section{WITH PRACTICAL EXERCISES}

llach

BY

W. M. BAYLISS, M.A., D.Sc., F.R.S.

profeESOR OF GENERAL PHYSIOLGG IN UNIVERSTY COLLEG, LONDON

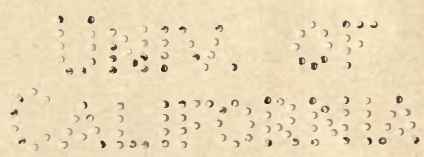

LONGMANS, GREEN, AND CO.

39 PATERNOSTER ROW, LONDON, E.C.4

FOURTH AVENUE \& 30TH STREET, NEW YORK

BOMBAY, CALCUTTA, AND MADRAS

1919

All rights reserved 


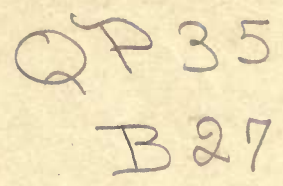

BIOLOGY

LIBRARY

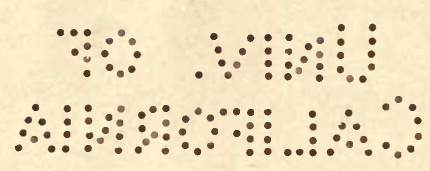




\section{PREFACE}

To write a satisfactory text-book for those commencing the study of a science is well known to be of greater difficulty than to write one for advanced students. In fact, it would probably be true to say that the former task is a severe test of the author's comprehension of the subject. I venture to hope that the thought given to the present work may not be found to have been in vain. Any readers who may find parts of it expressed insufficiently clearly are requested to help the author with suggestions.

It was found to require much consideration to decide what should be omitted. My firm conviction is, that a thorough understanding of the main fundamental conceptions is of more value than a superficial acquaintance with a large number of facts and theories, even if it leads, for the moment, to the omission of what many may regard as essential. The student will probably be surprised to find how many of the more detailed descriptions follow naturally from a knowledge of a few general principles, when these are clearly grasped. For this reason, more space has been given to certain elementary facts of physics and chemistry than might be supposed necessary; but it has been my experience that, although a student may have attended good courses in these sciences, he does not readily apply the knowledge to physiological problems. Moreover, there are some things essential to physiology, but often regarded as outside the scope of an elementary course in the preliminary sciences. I intentionally lay myself open to the charge of inserting matter that is not, strictly speaking, physiological.

The rather "intensive" treatment sometimes adopted leads to what some may regard as an excursion into advanced regions. But this is unavoidable when the conception cannot be passed over without risk of error.

In another way, the manner of presentation adopted may meet with objection. Students do not easily remember the names of the 
discoverers of the facts they must learn ; and, when they do associate names, they are frequently incorrect. Naturally so, since the discoverers are, as a rule, mere names to those commencing the study of the subject. For this reason, names, for the most part, have been omitted, with the exception of those of a few outstanding men of genius, such as Faraday, Claude Bernard, or Ludwig, which ought to be familiar to all who claim to be educated. I would therefore ask pardon of my colleagues who may find their discoveries referred to without their names. At the same time, I have not hesitated to refer to any personal or historical fact which might give added interest to a dry description. Similarly, the only references given are to my own larger "Principles," where those desirous of more detail can find what they require, or, at any rate, indication of where to find it. These references have the letter $P$ prefixed to the page indicated.

It is to be feared that the exposition may be regarded as too dogmatic. This may be so, but it is of set purpose, and the arguments have been carefully weighed before making a statement. Where there is obviously insufficient knowledge, it is preferable to state that this is the case, rather than to confuse the student by conflicting views. In my early student days I was repeatedly presented with arguments on both sides, about as many on one side as on the other, so that a blank remained, not only in the note-book, but in my memory. If only a view is clearly grasped, it can easily be corrected later, if necessary, since the point where it fails can be seen and understood.

On the whole, it would have been preferable to leave details of experiments to be shown to the student, or made by himself, to the discretion of the individual teacher, in accordance with the resources of his laboratory. But it has been represented to me that the value of the book would be much increased if such details were included. This has accordingly been done. As will be seen, however, the experiments vary a good deal in their simplicity. Some of them require apparatus that a particular laboratory may not possess, and must perforce be omitted. On account of their importance, nevertheless, it would have been misleading to omit them on the ground that the necessary means may not always exist. The value of experiments, especially when made by the student himself, is very great. They give a reality to description in words and a belief in 
the truth of the statements made. There is of necessity much that requires more difficult and lengthy work than is possible in the time available for class work, since many of the most fundamental facts could only be discovered by methods involving the greatest accuracy in measurement.

The instructions as to experimental work are intended to assist the teacher as much as the student. As already remarked, much must be left to individual discretion. Probably instructive experiments will occur to the teacher in addition to those given, and I shall be very grateful for suggestions to be included in a future edition. There are doubtless many shortcomings in this manual. Those statements in the text which are capable of experimental illustration are marked with the letter $\mathrm{E}$, and the page of the "Practical Work" on which instructions are given.

It seemed scarcely possible to add summaries to the chapters, as in my larger book, for the reason that all the matter contained in so small a space is of nearly equal importance, and a summary would have been almost as long as the chapter itself. It would also tend to encourage what I wish most to avoid, namely, any kind of merely learning by heart. The student may find it profitable to make abstracts for himself.

Structural facts, whether anatomical, histological, or chemical, are not given with more minuteness than necessary to understand the mode of action of the organs they apply to, so far as it is known. If further description is thought useful, such books as Quain's "Anatomy," Schafer's "Essentials of Histology," and Plimmer's "Practical Organic and Bio-Chemistry," may be consulted.

The best way in which the present book could be used would be for the teacher to take it as a suggestion of what the author regards as the fundamentally important things to be taught, and to describe them to the student in his own words. The student may use the book to remind him of what he has been taught, or to obtain a different way of looking at the phenomena. In this way, that most pernicious habit of learning a subject, already alluded to, may be avoided, to some extent at least. The committing to memory a mass of statements without understanding their relation to one another, or even what they mean, cannot be too carefully guarded against. It is of no value whatever, either as a means of education or for future use. 
In justice to the student, it should be pointed out that the requirements of no particular examination have been taken into consideration here. It is to be hoped that the day is not far distant when preparation for an examination, other than periodic questions by the teacher himself, will be a thing of the past. It is, no doubt, difficult to devise a method of replacing examinations as a test for such purposes as medical qualifications, but it is surely not impossible. Many of the existing examinations can be passed without much real knowledge, while the preparation of students for examinations, when these are conducted by others than the teacher himself, has a most depressing effect on those who have to do it. However important a new discovery may be, hesitation is naturally felt in taking up the students' time with what they know will not help them to pass their examination.

Some explanation is needed as to the diagrams given here. I venture to think that, at all events in many cases, a picture which attempts to represent what a part of a living organism actually looks like is less instructive than one which frankly attempts no more than to indicate what is essential to the working of the mechanism. The real appearance can only be learned from actual specimens and experiments. Such an excellent book as the "Practical Biology" of Huxley and Martin shows that illustrations are not a necessity.

W: M. BAYLISS.

UNIVERSITY COLLEGE, LONDON. 


\section{CONTENTS}

\section{PART I.-TEXT \\ CHAPTER I}

LIFE AND ENERGY

The Problem

PAGE

Protoplasm -

Brownian Movement and the Ultra-Microscope -

The Kinetic Theory

Amœboid Movement and Surface Tension

Heterogeneous Systems and Boundary-Surfaces

Energy and its Laws

The Cell-Membrane

The Permeability of the Membrane

Osmosis

Osmotic Pressure.

Electrolytic Dissociation -

Strength of Acids and Bases

Indicators

Electrical Resistance of Living Cells

Changes in Permeability during Life

The Colloidal State -

Precipitation by Electrolytes -

Electrical Adsorption and Staining

Hydrolytic Dissociation

The Nucleus of the Cell

Mitochondria

$\begin{array}{llllll}- & - & - & - & - & 10 \\ - & - & - & - & - & 15 \\ - & - & - & - & - & 16 \\ - & - & - & - & - & 17 \\ - & - & - & - & - & 21 \\ - & - & - & - & - & 23 \\ - & - & - & - & - & 26 \\ - & - & - & - & - & 27 \\ - & - & - & - & - & 28 \\ - & - & - & - & - & 29 \\ - & - & - & - & - & 30 \\ - & - & - & - & - & 35 \\ - & - & - & - & - & 36 \\ - & - & - & - & - & 38 \\ - & - & - & - & - & 39 \\ - & - & - & - & - & 39\end{array}$

CHAPTER II

\section{FOOD-DIGESTION AND RESPIRATION}

Composition of Protoplasm

Source of Carbon--The Sugars

Source of Nitrogen-The Amino-Acids

Optical Activity

The Green Plant

The Cycle of Nitrogen

Sulphur and Phosphorus

Salts 
Accessory Factors -

The Supply of Energy

Digestion

Enzymes

Changes in Carbollydrates -

Fat

Proteins

The Large Intestine

Movements of the Alimentary Canal

Secretion

Respiration -

The Lungs -

The Mechanism of Oxidation

CHAPTER III

WORK-THE MUSCLES

Length and Work -

Gradation of Contraction. "All-or-Nothing"

Refractory Period

Staircase

Voluntary Contraction

Various Muscular Mechanisms

Posture Phenomena

Energy for other Purposes -

Maintenance and Regulation of Temperature

CHAPTER IV

\section{STIMULATION-THE SENSES}

Receptors in General

Pain -

Touch

Heat and Cold

Taste and Smell

Hearing

Sight

Position Receptors -

Proprio-Ceptors

CHAPTER V

\section{ADJUSTMENT-THE NERVOUS SYSTEM}

The Reflex -

The Neurone

Reflex Action

Reciprocal Innervation 
CHAPTER VI

\section{TRANSPORT OF MATERIALS-THE VASCULAR SYSTEM}

General Arrangement

The Blood -

Internal Secretions -

The Kidneys

Lymph

The Proteins of the Plasma

The Salts of Blood

Viscosity

The Regulation of the Blood Supply

The Capillaries

The Regulation of the Heart Beat -

Origin and Transmission of the Heart Beat

PAGE

132

I35

136

137

140

I4 I

142

144

145

147

148

150

CHAPTER VII

GROWTH AND REPRODUCTION

Fission

Conjugation

I 5 I

152

Sexual Reproduction

Heredity

Variation

Adaptation -

Struggle for Existence

\section{PART II.-LABORATORY WORK}

\section{CHAPTER I}

The Microscope

Nature of Protoplasm

The Leucocytes of the Blood .

Movement of Protoplasm

Dark-ground Illumination

Brownian Movement

Surface Tension

Adsorption -

Cell-membrane and Permeability

Osmotic Pressure

Blood Corpuscles

Plasmolysis

Turgor -

Contractile Vacuole

Direct Measurements of Osmotic Pressure 
The Colloidal State

Colloidal Gold -

Emulsoids

Swelling -

Surface Tension and Dispersion

Electrical Charge

Action of Electrolytes -

Mutual Precipitation

Staining and Electrical Adsorption

Rate of Reaction between Colloids

Chemical Composition of Organisms

The Polarimeter

Waste Products

Carbon Cycle

Water Culture -

Action of Green Plant on Carbon Dioxide

Chlorophyll

Formation of Starch

The Nitrogen Cycle

Bacteria

Formation of Nitrates in the Soil

Salts

Root Nodules

Sources of Energy

Alimentary Canal of Frog and Rabbit

Enzymes and Digestion

Rates of Reactions

Hydrolysis by Enzymes

Enzymes Act at their Surfaces

Catalytic Action Model

Various Digestive Enzymes

Amylase

Invertase -

Pepsin

Absorption

Trypsin

I8I

I8I

I 82

183

183

183

186

I 86

I 86

186

I 86

I 87

187

189

I 89

I 89

189

190

192

192

192

193

I93

I93

Histological Preparations

Voluntary and Involuntary Muscle

Pancreas

Living Newt's Stomach

Flow of Water -

Electrical Change

Respiration -

Tracheæ of Insect

Hæmoglobin as Oxygen Carrier

Stimulation of Respiration by Carbon Dioxide 
Jxidation

Autoxidation

Peroxides

Peroxidase

Guaiacum Reaction

Reduction by Milk

CHAPTER III

Action of Jointed Bones

Contraction of Muscle

Tension in Muscle -

Spring

Nerve-Muscle Preparation -

Formation of Acid -

Effect of Length of Fibres

Structure of Voluntary Muscle

Effect of Temperature

Production of Tetanus

Heart Muscle

Staircase

"All-or-Nothing"

Refractory Period

CHAPTER IV

Spinal Frog

General Anatomy of the Nervous System -

Nerve

\section{Structure}

Electrical Change

Unexcited by Light or Sound -

Receptors of the Skin

Taste-Buds

Olfactory Cells

Heat and Cold Spots

Hearing

The Eye

Visual Purple -

Anatomy

Image on the Retina

Structure of the Retina

Receptors for Position

Statocyst of Cyclas

Semicircular Canals of Skate

\section{CHAPTER V}

Anatomy of Central Nervous System of the Frog - 


\section{CHAPTER VI}

Model of the Circulation

The Circulation in the Frog's Web

The Heart of the Sheep

Blood

The Kidney -

The Salts of the Blood

Function of Bicarbonates

Vaso-Motor Effects

Action of Drugs on the Heart

The Beat of the Heart

\section{CHAPTER VII}

Dividing Nuclei

Development of the Frog -

Ova and Spermatozoa

The Structure of a Flower -

Process of Fertilisation in the Plant 
AN INTRODUCTION TO
GENERAL PHYSIOLOGY

PART I 



\section{CHAPTER I}

\section{LIFE AND ENERGY}

\section{The Problem}

WE all know what living beings are, and it would be unprofitable to attempt to define life in such a way as to make an inhabitant of another planet understand what we mean by it on the earth. The most striking thing in the behaviour of living things is their perpetual change-they are always doing things. From that aspect, with which physiology has to deal, we may say that they are extraordinarily complicated machines, in which the laws of physics and chemistry are made use of in a way quite different from that in which a machine made by an engineer uses them. What we have to do, then, is to try to find the way in which living machines work (P., p. viii).

It is natural that our own bodies should be, to ourselves, the most interesting and important of living organisms; but when we come to investigate them, we find that there is nothing in them which is not to be found in some form in what we call the lower organisms. Certain arrangements, especially those connected with the brain, are more complex, it is true. These would not be understood, however, without a knowledge of the simpler arrangements to begin with.

It must not be forgotten that physiology is not directly concerned with the mind. Our thoughts and feelings, when investigated with the view of finding out how they depend on one another, is the province of another science, psychology. So that when we speak of living machinery, it is not to be supposed that a denial is made of the existence of anything else. When the functions of the brain are discussed, it is the changes taking place therein, as looked at from the outside, that we are dealing with.

Plants, as well as animals, are alive, and we shall find that there is very much in common between them. Strictly speaking, 
"general" physiology should be confined to those properties which belong to all living creatures. But this is not the meaning taken here, because it would give us too limited an outlook. It is really impossible to make a distinction between general and human physiology. Perhaps the best way to explain the difference is that it is the manner in which the subject is treated, rather than the subject-matter itself. Thus, instead of taking an organ, such as the liver, and talking about all the different things that it does, we intend to discuss the processes in which it plays its part along with other organs. This will become clearer as we go on, together with the fact that whatever name we give to the particular form in which the phenomena are presented, they are themselves always the same.

Just as physics and chemistry deal with the laws of inanimate nature, apart from and necessarily before their application to practical industry, so there is a body of science dealing with living nature, which is a necessary preparation for application to the human body. But it would exist as a science quite independent of any such application. The reader may be reminded, especially if he is inclined to overrate the importance of what is obviously of immediate practical use, that it is not possible to know beforehand what and when pure scientific knowledge may become suddenly of the greatest practical value. Electric waves in physics, and the electrical phenomena of the heart in medical science, may be taken as two of the numerous instances of the kind.

\section{Protoplasm}

Like every other machine, a living organism does its work because it is made in a certain way. We must know its structure, therefore. And, first of all, let us see what is the simplest structure that we can call "living."

There are some minute creatures that consist only of a substance which has the appearance of a clear jelly, at first sight. But when we look at it more closely under the microscope, we see that it changes its shape, whereas a little lump of jelly would not do so. This material, which is known as "protoplasm," behaves more like a drop of oil in water, except that it seems to have the power of movement on its own account.

The organism called Amoba is composed of such protoplasm, but it also contains some other things floating in its body-substance (E., p. I64). The "nucleus" may be noticed particularly, a spherical structure in one part or other of the protoplasmic mass. There are also particles and drops of liquid of various sizes and 
shapes. Neglecting these for the present, we will examine the clearest part of the protoplasm. The very clearest part of all will be noticed when a part of the protoplasm makes a protrusion, called a "pseudopodium," because it serves the purpose of a leg, and causes the animal to move along. In this part it will be difficult or impossible to see any structure at all, a fact which shows that the visible grains and so forth are not essential constituents of living material.

The whole organism, protoplasm and nucleus, is known as a "cell." The name does not seem very appropriate in this particular case, but it was first applied to the constituent parts of vegetable organisms, in which what corresponds to the whole amœba is enclosed in a box of material which is not itself living. Under certain conditions, amœba itself forms a coating around itself and becomes quiescent.

The larger and more familiar plants and animals are composed of a great number of cells, joined together in a community for mutual help. Some of them do one thing, some do other things; whereas, in the unicellular beings, the one cell performs all the functions of which the organism is capable. This fact, of course, makes it practically impossible to discover much from observations on these creatures. But we can find out many of the fundamental and necessary properties belonging to all living cells.

Apart from the fact that what we see in the moving protoplasm of an amœba conveys the unavoidable impression that it is liquid, there are other facts which confirm this (P., p. 6). But before we proceed to these facts, it should be pointed out that, so far as can be made out, protoplasm sometimes sets into a jelly and ceases to be liquid. This is a temporary state, and the liquid condition returns again, as when an ordinary jelly is warmed (P., p. 19). In protoplasm, the change from one state to the other occurs without altering the temperature. This process will be better understood after we have learned something about what is called the "colloidal state."

\section{Brownian Movement and the Ultra-Microscope}

If we look at the smallest particles which we can see in protoplasm by the use of a fairly high magnification with the microscope, we notice that they are in a perpetual kind of dancing movement. This can also be seen with any small particles suspended in water. The yellow resin, gamboge, used as a water colour, rubbed up in water, shows it very well (E., p. 166). The name of the form of 
movement we are considering has been given to it on account of its first description by the botanist, Robert Brown. It will be seen that the smaller the particle, the more lively its movement. There are difficulties in seeing the very minute particles, because they are so small. Now, when such particles are brightly lit. up and looked at against a dark background, they are much more visible. A ray of sunlight entering a dark room through a crack shows up a myriad of bright particles when we look at it from the side against a dark wall, whereas the surrounding air appears quite empty. This is the principle of that method known as the ultra-microscope or dark-ground illumination (P., pp. 79-\$2). The rays from a bright lamp are sent sideways through the slide on the stage of a microscope, so that they do not enter the objective, and, if there were nothing on the slide, one would only be aware of darkness. If, however, anything solid, able to reflect light, were there, it would be lit up and send rays in all directions, becoming a visible object. The particles of gamboge should be examined first, and afterwards the living amœba (E., p. 166). The brighter the illumination, the smaller are the particles which can be seen. If it is sufficiently intense, it will be found that even in the clearest protoplasm there are particles to be seen.

The existence of these movements in the particles contained in protoplasm shows that they must be free to move. In other words, they are suspended in a liquid. In a solid mass, even with the properties of a jelly, they would not be free to move. The experiment can be made with gamboge in gelatin (E., p. 167).

\section{The Kinetic Theory}

The explanation of Brownian movement requires a few words on the constitution of matter, as now generally accepted.

If we imagine a crystal of common salt to be divided up into smaller and smaller fragments, we should find that at a certain stage, which could not be arrived at merely by mechanical crushing, although possible by dissolving in water, the separate fragments are such that any further division changes their chemical properties, and there are now two things present of different nature. The particles which are the smallest possible without alteration of chemical properties are "molecules ;" the two different substances, arising from further splitting, are "atoms," and, as the reader is doubtless aware, are sodium and chlorine. It was at one time believed that atoms were incapable of further decomposition, but the study of the radio-activity of certain "elements," and that of the phenomena of the electric discharge, have taught us that atoms 


\section{LIFE AND ENERGY}

are themselves complex organisations, and sometimes divide up into other smaller elements.

Molecules are not always composed of different kinds of atoms; two or more of the same kind may be united together, as in the case of those materials called chemical elements, in the free state, such as the oxygen and nitrogen of the atmosphere, iron and copper, and so on.

Now, suppose that we consider how these molecules are behaving in a gas such as the atmosphere. It is clear from the fact that we can, by pressure, make a particular volume into a smaller one, as, for example, by pushing in the piston of a syringe with the nozzle closed, that the molecules cannot have been in close contact originally. They must have an actual size and, therefore, there must also be free spaces between them. The molecules, indeed, make up a very small part of the total volume of a gas. A rough idea of how little it is could be obtained by taking a flask full of the vapour of water and cooling it, so that the steam is condensed to water. The total number of molecules must be the same in both steam and water, or, more correctly, the number of atoms must be the same in both, since we shall see later that some of the molecules combine together when steam condenses to water.

Why, then, do we have to exercise pressure on a gas if we wish to make its volume smaller? Why does it resist the process? It is because the molecules are in a state of perpetual to-and-fro movement, hitting against the vessel containing the gas with a total pressure in proportion to the number of molecules that hit in a given time. If we diminish the volume, we press more molecules into the space than were previously there, so that we increase the number of hits. Although these molecules hit against each other occasionally, they are practically free from anything to hold them together, so that, if a vessel containing a gas is connected to another empty one, the gas divides itself equally between the two. This movement of the molecules is due to their possession of that form of energy which we call heat.

In a liquid, the constituent molecules are so close together as to be within the distance at which they begin to attract one another. Although this attraction does not begin to be appreciable until the molecules are extremely near together, it reaches a very high value at that position; so that a very great force is required to pull them further apart. The attractive force between molecules shows itself as cohesion, and, in the case of a liquid, is known as the internal pressure of that liquid, with which we shall meet again presently. The molecules of a liquid cannot, then, move further apart from each other, but they can rush about with a movement like that of the molecules of a gas, so long as their distance from 
the molecules among which they move does not increase. On the other hand, the molecules of a solid are not free to move about; they can only vibrate backwards and forwards about the same mean position.

The attractive force between molecules is doubtless due to the structure of the atom as consisting of electrically charged smaller constituents, "electrons," with negative charges, moving in various kinds of orbits around a positively charged central body.

In a liquid, then, the molecules are in constant movement, hitting one another and rebounding. If a solid particle, large enough to be hit by many molecules at the same time, be immersed in water, the resultant force acting upon it will either be zero or very small, because there will be about the same number of hits in one direction as in the opposite one. The mass, again, of a large particle would require to be hit in the same direction by a large number of water molecules at the same time in order to move it. But, if the particle is small, while still large enough to be visible when adequately illuminated, it will be exposed to unequal bombardment in opposite directions, and receive enough impulses to send it moving until it is met by impacts sending it in another direction (P., p. 86). This is Brownian movement, and we see that it is a true representation of the molecular movements in the liquid itself, so that, by looking at it, we get an idea of the way molecules are in movement, as stated by the kinetic theory of gases and liquids.

\section{Amœboid Movement and Surface Tension}

We have noticed already how an amœba moves about by means of a local protrusion of a part of its protoplasm and the drawing up of the other part of the organism. The movements of the protoplasm in a plant cell, such as one of those making up the hairs on the stamens of Tradescantia, should also be examined, as showing another form of protoplasmic movement (E., p. 166). Now, while it would be rash to state that the process can be completely explained in a simple way, there is no doubt that what is known as "surface tension" plays a large part in it. Since this property has important relations to numerous physiological phenomena, we must give some attention to it.

First of all, let us convince ourselves by some experiments with a soap bubble or film of soap solution that the film behaves as if it were stretched (E., p. 167). The fact that a drop of oil suspended in a liquid of its own specific gravity, so that it does not rise or fall, takes a spherical shape also serves to show that the surface of the 
drop is in a state of tension. The surface takes that form in which its area is the smallest possible, that is a sphere (E., p. 168).

The surface of an amœba is, then, in a state of tension, and if the tension were the same everywhere, it would be a spherical drop, like the oil. It does, indeed, become of this shape when stimulated by an electric shock (E., p. 165). We will suppose that it is, at a particular moment, spherical, and that at one part or another something happens, either inside the organism or in the water outside it, which makes the tension less at this spot. It will be clear that the greater tension on the remaining part, which tension, of course, produces a pressure throughout the liquid protoplasm, will result in a pushing out of that part of the surface where the tension is less. Thus a pseudopodium is formed.

Here we may note a further proof of the liquid nature of protoplasm. If the drops of liquid, which are usually present in an amœba, sometimes containing organisms taken as food, be observed, they will be seen to be spherical, whatever the shape of the material inside them (P., Fig. 3, p. 2). The surface of the liquid is free to take the form required by its surface tension. A drop of fluid imprisoned in a jelly may be of any shape whatever.

But how is this surface tension to be explained?

What, however, do we mean by "explanation" in science? We have already "explained" Brownian movement by the kinetic theory of gases and the formation of pseudopodia by surface tension, and what we have actually done is to show that these complex things are special cases of properties possessed by very much larger groups of existences, not necessarily living. When we now proceed to "explain" surface tension itself, what we do is to show that it is a consequence of the properties possessed by liquids as such. A further step might be to refer these properties back to those of the molecule itself. It will be clear, nevertheless, that scientific explanation must stop sooner or later. Even suppose that everything has been explained in terms of the movement of electric charges, this movement itself still remains a mystery. But science does not pretend to be able to go beyond what can be investigated by the powers we possess.

As physiologists, our task is to refer, as far as we can, all phenomena of life to the laws of physics and chemistry. At present we have to be content, in many cases, with a reference to more general physiological laws, applying to a larger group of phenomena than the particular ones under consideration, but themselves still "unexplained."

And now we may proceed with that task. 


\section{Heterogeneous Systems and the Phenomena at their Boundaries}

A glance at any living organism is sufficient to impress upon us the fact that it is composed of a great variety of things that are distinct from one another in space. In the amoba, for example, the nucleus and the particles scattered about in the protoplasm do not mix with the rest of the cell substance. Moreover, we have seen that even the clear part is full of tiny particles. The individual cells, as well as the whole organism of a higher plant or animal, are what the chemist would call "heterogeneous systems," as contrasted with such systems as solutions of salts in water. If we take a sample from any part of a solution of common salt in water, we find it to have the same composition. It is a "homogeneous system." It could be made heterogeneous, however, by the addition of a solution of silver nitrate. The precipitate of silver chloride could be separated from the liquid. If we divided up a living cell into parts, these parts would not have the same composition.

The various parts of a heterogeneous system-the parts that do not mix with one another-are called phases. The name might seem to imply that they have the same chemical composition, and this is sometimes the case. Take, for example, ice floating on water at the freezing point. They are separate phases, with the same chemical composition. But this is not necessarily the case. Charcoal, suspended in water, forms one of the phases of this twophase system. There are certain laws which control the behaviour of heterogeneous systems, some of which we may briefly consider here. Others will be met with later.

Consider water in a basin. The molecules in the depth of the water are exposed on all sides to the influence of molecules like themselves, not only in chemical nature but in their state of motion, etc. They are attracted equally in all directions. This attraction, as we saw before, gives rise to the "internal pressure" of the liquid. Those molecules at the surface, on the contrary, are only exposed to the attraction of similar molecules on the one side; the other side is exposed to air, where the molecules are very few in number, and not limited as regards their distance from one another. There is, as a result, a continual force exerted on the water molecules at the surface, trying to pull them down into the liquid. This could not happen, of course, without diminishing the volume of the water, and even then there would always be molecules at the surface. But the molecules are so close together in a liquid that they cannot be made to get closer except by enormous pressure, or by decreasing their kinetic migrations by cooling them. The result of the pull inwards can 
only be that the surface takes the smallest area possible to it, and resists any attempt to make it larger. In other words, it behaves as if stretched.

It will also be clear that the molecules at the surfaces where any unlike substances touch one another are similarly exposed to forces different from those in the interior of the substances.

For the present, we are only concerned with that aspect of dissimilar forces at the surface which results in surface tension, a phenomenon which we can only detect when the molecules are free to move, but unable to get away from the influence of their neighbours; that is, at the contact surface of liquids with gases or other liquids. We can detect it indirectly at the contact of liquids with solids, and there must also be related phenomena at the contact of solids with cach other and with gasses. It will be clear that there cannot be anything of the kind with gases themselves, because their molecules are completely free to wander away into the interior of both, so that gases in contact always mix up together. In other words, if gases form a part of any heterogeneous system, they can only form one phase, however many different chemical species this phase may be composed of.

The body-substance of an amœba, as is easily seen, does not mix up with the water in which it lives. It forms a separate phase, just as oil and water form distinct phases. But we know that protoplasm consists largely of water, as can be seen when it dries up and returns to life again when moistened, as sometimes happens. Moreover, chemical analysis shows it to contain 80 per cent. or more of water. We have seen that it is a liquid, so that it must be a solution of various things in water, and it contains also other things floating in it. If an amœba is killed by a strong electric shock (E., p. 165), its protoplasm is dissolved up and disintegrated by the water around it. Why, then, does it not mix with water in normal conditions? It must be surrounded by some kind of a layer that protects it. We have to find out how such a layer or film, sometimes called the " plasma- or cell-membrane," is produced, taking into account the fact that it is not a permanent rigid case, like the cell wall of a plant or the shell of an egg. This is obvious enough from observation of a pseudopodium. As it is formed and increases in size, there is no mixing of its substance with the water. Hence the membrane must be continually being produced at the contact between water and protoplasm. We shall see later, moreover, that a dye, such as aniline blue or congo red, is unable to pass through the cell-membrane, and that it is equally unable to pass into a bit of protoplasm cut off from the main mass, although, when either is killed in any way, the dye freely enters. 
The difficulty will probably occur to the reader that solid particles, such as the bacteria and algæ used for food, enter an amœba, although solutions of dyes do not. The difference is due to this very fact of the latter being. solutions. They can do no mechanical violence to the membrane, whereas a solid particle breaks through. The hole in the membrane, however, is mended as soon as the particle has passed. What happens is like the dropping of a needle through a soap film. When the point touches the film, it becomes covered with a continuous film, which prevents an actual break. As the needle passes through, before the eye-end has left the film, it also has a film over it, which is left behind as the needle drops through.

It may be pointed out here that the substances resulting from the digestion of the food of the amoba are freely dissolved by water. They would quickly be washed out if they could pass through the membrane, and so be lost to the organism.

In order to understand how such a membrane could be formed, we must direct our attention to the doctrine of energy, especially in certain aspects. This is, in any case, a necessary preliminary to further study.

\section{Energy and its Laws}

Living beings are always doing something, making changes in their surroundings. This means work. When we have done work, we feel that we have lost something that has enabled us to do the work. Now, this is called "energy," and is actually defined as the capacity of doing work. Moreover, it can be accurately measured and shown to be exactly equal to the work done. Since energy is not a thing to be seen, apart from the material bodies possessing it, we are rather apt to overlook its importance in ordinary life. The chief use of the food we take is to supply us with energy. If it were merely to make bodysubstance, flesh or bones, we should need very little indeed. A certain quantity of any particular food-stuff contains a definite amount of energy, no more and no less, and will enable a certain amount of work to be done, no more and no less.

The reader is familiar, no doubt, with the two great laws at the foundation of the doctrine of energy. They are usually known as the First and Second Laws of Thermodynamics; but it would be better, especially from our physiological point of view, to speak of them as laws of "Energetics," since they apply to all forms of energy. They were first established by investigations of that form of energy known as heat, hence their usual designation.

The words, "forms of energy," just used, imply that energy may 
be of various kinds. Let us consider for a moment what sorts of properties objects may possess in virtue of which we can get them to do work for us. A bullet is a very different thing lying on the table from what it is just after it has left the rifle barrel. In the latter case, it can do work because it is moving; it is said to possess "kinetic energy." A reservoir of water at a high level can do work as it falls to a lower level, as through the turbine or over the mill-wheel. This is due to gravity. If the water remains dammed up, the energy is there, but not in use; we say that it is potential. The fire under a steam boiler makes the engine do work-we have heat energy. The current of electricity in an electro-motor enables it to drive machinery. The rays we receive from the sun, some of which we call light, do an immense amount of work. We may call this radiant energy. One of the most important sources has not yet been mentioned. That is, the energy of chemical combination. Certain chemical substances; when they combine together, give off energy in various forms. These substances must originally have contained it in a potential form. Consider the petrol of an internal combustion engine. It gives off energy when it combines with the oxygen of the air. The products, carbon dioxide and water, contain no energy that is available for use. The greater part of the chemical energy that we meet with is derived from combination with oxygen, which we call combustion. The energy of our own bodies has the same origin; we burn up our food by means of oxygen obtained from the air. The fact that one form of energy can be converted into others is very obvious in this case. In the steam engine, the combustion of the fuel comes out as kinetic energy. If the engine drives a dynamo, the energy of the combustion appears in part in an electrical form. We obtain heat from our house fires, and light from the burning of candles or from the electrical current. And we can convert our electrical current back again into chemical energy by the decomposition of water or by the use of the storage battery.

The first law of energetics is the expression of the fact found to be true whenever it is tested, namely, that any form of energy can be converted into any other form of energy, and that there is no loss and no gain in the process. This is always found to be true. If we measure accurately the amount of energy, supplied to a motor by the current, and also that which is given out by it as mechanical work, together with that appearing as heat in the motor itself and the other parts of the arrangement, we find them exactly equal. Similarly, if we compare the chemical energy of the fuel burnt in a petrol motor with the mechanical work done and the heat produced, we find them equal. 
Now, one of the most significant and important results of modern physiological investigation is that this first law has been shown to apply to the human body itself. The amount of chemical energy taken in the form of food can be measured, and that given out in different forms can be converted to heat. When this is done, the balance is found to be so close as to be practically perfect.

Although, as we have said, there is no loss in the conversion of any one kind of energy to any other kind, so far as the final total sum is concerned, there is a certain limitation in the case of heat. With this circumstance the second law of energetics deals. This law may be looked at in two ways. In the first place, it expresses the fact that, while any other form of energy can be completely converted into heat, heat itself, under the conditions in which we live, can only be partially converted into other forms of energy. The proportion is given by the well-known formula relating the fall of temperature along which the work is done to the actual height of the temperature above a particular point, at which heat energy is absent, called the absolute zero of temperature. How do we find out where this zero is? Take a volume of a gas at $0^{\circ} \mathrm{C}$. Owing to the heat energy present in it, the molecules are in a state of movement, and sufficiently far apart that the volume taken up by them is so small as to be a negligible fraction of the total volume. Lower its temperature by one degree. The kinetic energy of the molecules is reduced, so that the volume taken up by the gas is diminished, if we keep the pressure from altering. This diminution in volume is found to be $1 / 273$ of its initial volume. Hence, if the temperature is lowered by $273^{\circ}$, the volume will be reduced to nothing, provided, of course, that nothing happens to change the nature of the gas, and that we disregard the volume of the molecules themselves. This temperature is the absolute zero at which heat energy is absent altogether; the kinetic energy of the molecules has disappeared. In actual fact, of course, the volume cannot decrease beyond that point at which the molecules touch one another. The reason why heat has the peculiar position as regards conversion to other forms of energy is, therefore, because the temperature at which we work is so far above that at which heat energy is absent. We can never completely get rid of it; whereas we can have a total absence of mechanical, electrical, or chemical energy.

The other aspect of the second law is that of free energy. We have seen that the various forms of energy, with the exception of heat, can be entirely converted into other forms; and, so long as they are not changed into heat, we may be said to be free to use the whole of them. But, if ever we allow any of this free energy to be "degraded," as is often said, to heat, we can only use a part 
of it again to do work for us. Since free energy is continually being converted into heat in all sorts of processes going on, it is clear that the free energy of the universe is steadily decreasing. This fact was pointed out by Lord Kelvin and called the "dissipation of energy," that is, of free energy. The energy that is lost in this way has been given various names, "bound" energy, as distinguished from that which is free, sometimes "entropy." The last name is used when we wish to give a quantitative measure of the fact, and we say that the entropy of a system is the ratio of the bound energy to the absolute temperature. That energy may be present, but in such a form that we cannot make use of it, may be grasped by imagining that we have a hot ball of metal and a cold one, so insulated from their surroundings that no heat can arrive or escape. A certain amount of heat energy is present, and by an appropriate mechanical device we can obtain useful work as the heat passes from the hot to the cold body. But, as soon as the two objects have reached the same temperature, one falling, the other rising, no more work can be got, although the total amount of heat present is unaltered, since none has entered or left.

In this connection there is an important fact to be remembered, a fact for which no reason can be assigned, but which is one which has never been found to be otherwise. In the present universe, free energy always tends to become "bound," if it is possible for it to do so. If one may so express it, it takes advantage of every opportunity of losing its freedom. It is not impossible to imagine a state of things, otherwise similar to that which we know, where free energy would tend to increase; but it is not so as matters are now arranged.

There are three consequences of this second law which are of special interest in regard to the phenomena of living organisms. It has been pointed out that the so-called struggle for existence is really one for the possession of free energy. There is unlimited heat energy in the objects around us. What we demand is the energy which is continually reaching us from the sun, and is converted into the chemical energy of our food by the aid of the green plant, as we shall find in the next chapter. The second point is that, for the economical use of the energy we get from our food, it is important that it should be converted into the other forms we require, say that of muscular movement, without passing through the stage of heat. We shall see later that appropriate means are taken to ensure this. The third point is that it enables us to predict many things that happen. If we find out that a process is associated with a decrease of free energy, we have every reason to reckon upon its taking place, whenever it can. An instance of this will be seen immediately. 
We may pause for a moment to point out that an explanation of a phenomenon as a consequence of the laws of energetics does not tell us about the mechanism by which it is effected. This must be on the basis of the kinetic theory and the structure of atoms and molecules.

The statement made at the beginning of this chapter about the property of living beings to produce changes may now be made somewhat more precise. It is in the process of change of one form of energy into another that the phenomena especially characteristic of life make their appearance. When this change ceases, or, as it may be put, when equilibrium has taken place, we have a state of death. Just as in commerce, money that is unemployed is of no value.

The boundary surface of a liquid being in a state of tension, it is clear that it may be made to do work. In a small way, the experiment that we made with the soap film in a funnel shows this; the film rises and lifts up its own weight. In this case, the tension in the small film is the same as that in the larger one, but the area is much less, so that the energy is less. We could also diminish the energy by reducing the tension without altering the area. The fact reminds us that there are two factors making up each kind of energy. One of these is always a sort of space or mass, and is called the "capacity" factor. The other is what might be called a strength or "intensity" factor. Some familiar instances will make the conception clearer :-

\section{Capacity Factor.}

Soap film - - Area.

Water power - - Volume of water.

Heat - - - Quantity.

Electricity - - Current (ampere).

Chemical energy - Mass of material.
Intensity Factor.

Surface tension.

Height above the earth.

Temperature.

Electromotive force or potential (volt).

Chemical potential.

Instruments for measuring these factors, with the exception of that of chemical potential, which is measured in a more or less indirect way, are in general use. This factor of chemical potential is not so easy to grasp as the others. It has been loosely called "chemical affinity," but it has clearly a real existence, as may be seen by the consideration that equal quantities of different combustible materials afford very different quantities of energy when burned with oxygen. And again, the chemical potential of oxygen and phosphorus is high enough for combustion to take place at a rapid rate, whereas oxygen and sugar only combine very slowly indeed, unless we raise the potential of the oxygen.

We may note in connection with chemical energy that there can be no doubt that there is a change in the internal structure 
and mechanism of an atom when it enters into chemical combination with another atom, and that it is in this way that energy is given off when, for example, carbon combines with oxygen, and that energy must be supplied when a chemical system of low potential is to be raised to one of higher potential, as when carbon dioxide is changed to sugar under the influence of the sun's rays.

There is one more point in connection with these two factors of energy. The quantity of heat energy in bodies of the same chemical composition at the same temperature is proportional to their mass. Thus, a litre of water at $100^{\circ}$ has twice the heat energy of half a litre at the same temperature. So that if we mix the two together we shall have three times as much heat energy as we have in the half litre, but there is no change in the temperature. The capacity factors, therefore, add together, while the intensity factors do not.

\section{The Cell Membrane}

We have seen that if we reduce the tension at a boundary surface, we reduce the free energy present. Now, nearly all substances when added to water have this property, and the degree to which the tension is reduced is in proportion to the amount of the active material present, up to a certain value. Therefore, suppose that there are things present in the protoplasm of an amoeba that lower the surface tension of water, the more of these that concentrate themselves at the contact surface of the organism with the water, the greater is the decrease of free energy. The second law of energetics tells us that this will happen. The name "adsorption" has been given to the process. Such an accumulation of a substance at the interface between two phases may go so far as to exceed the limit of solubility of the substance, so that it is deposited out of solution, and forms a more or less coherent or rigid membrane. The fact can be well seen by blowing a bubble with a solution of the vegetable product called saponin, which is not very soluble in water, but has a powerful effect in lowering surface energy (E., p. I68). Certain substances which we know to be present in protoplasm have properties like that under discussion. We should expect, therefore, to find them taking a chief part in the production of the cell membrane. These are especially those which have a fatty nature, and also the proteins, whose nature we shall learn in the next chapter. Fats have a particularly marked effect in lowering the surface tension of water. That at the contact surface between water and air is notably depressed by merely stirring the water with the finger.

The cell membrane is then to be regarded as a part of the 
protoplasm itself, and will vary in its composition, according to the chemical processes going on in the cell. Further, we must not forget that if the liquid outside the cell contains dissolved substances, these will assist in the formation of the membrane. This concerns especially the tissues of the higher organisms, which are bathed by solutions of a complex composition.

This phenomenon of adsorption is met with in a great number of cases, both in living organisms and in other heterogeneous systems. A familiar instance is the use of charcoal for removing colouring matters from solutions of other things (E., p. I68). The colouring matter is not destroyed by the charcoal, but deposited on its surface, whence it can be removed by appropriate means.

\section{The Permeability of the Membrane}

Having seen how the membrane is formed, we must next find out what are its properties, especially in view of what has been pointed out above as to the escape of matters from the cell. There are some things that it allows to pass, others not. Its "permeability" has to be investigated.

In the first place, it must allow water to pass through quite freely, because. we can see cells swell up under some conditions. What is the cause of this swelling is a rather difficult question, which must be discussed presently. But does the membrane allow anything which may be dissolved in the water to pass through? Unless the solute (that is, the substance in solution) is coloured, we cannot see directly whether it has gone in or not. But we can test the behaviour to coloured substances, such as aniline dyes and other pigments (E., p. I68). It is scarcely necessary to remark that we must not make use of anything that injures the cell, because the membrane would not then be in its normal state. Some aniline dyes can be used; aniline blue and congo red will be found not to stain the cell protoplasm. We see, then, that there are some solutes to which the cell membrane is impermeableav

In the cells of the root of the red beet, there is a pigment to which their membrane is impermeable (E., p. I68). But we can influence the membrane in such a way that it will allow the pigment to escape. Killing by heat does this. Certain chemical agents also do so. Moreover, some of these agents, if carefully applied, do not permanently injure, so that we can get the membrane to recover. This is important, because it shows the possibility of changes during life, so that at one moment a cell membrane may allow a substance to enter or escape, at another moment it may refuse passage to it, according to the state of the cell itself. The cane 
sugar which the cells contain can also be shown by chemical tests not to be washed out by water, as long as the cell is normal.

At the interfaces between different phases inside the cell, membranes must also be formed, and it is easy to see their importance in keeping separate the various reactions going on within a cell at the same time. The difficulty of finding out what is happening in the space of a single cell is very great, and we do not yet know much about it.

But, it may be said, supposing that the membrane is like a sieve, with holes through which such small molecules as those of water can pass, but which are too small for large molecules like the aniline dyes, and there are many reasons for believing that such is their structure (P., Pp. II 3, I I4), how does it behave to molecules which, although comparatively small, are larger than those of water, say sodium chloride? The greater number of these substances of physiological importance are colourless, so that some indirect way of testing the permeability of the cell membrane to them must be made use of. We have seen that the membrane is impermeable to cane sugar, and we need to test it as regards glucose and sodium chloride especially.

\section{Osmosis}

The most convenient way of doing so is by taking advantage of the phenomena of "osmosis" and their consequences. Here we come upon a property of solutions that is of some difficulty to explain and to understand. The reader may be reminded that there are different ways of looking at it, but that given below is probably the most intelligible to begin with.

Let us first make a few simple experiments to see what happens to red blood corpuscles when placed in water and various other solutions (E., p. 169). We take these bodies as convenient representatives of the cells of the higher animals, especially so for the present purpose since they are not attached together, and can be examined in the uninjured state with ease. Having a thin film of blood under the microscope, note the size of the corpuscles. Run in a Io per cent. solution of cane sugar. No change will be seen. This being so, we may dilute the blood with such a solution at once, a procedure which will render the observation of separate corpuscles an easier matter. Next, try the effect of a 5 per cent. solution. The corpuscles will swell up and may burst. This occurs so rapidly if water itself be used, that it is difficult to see what has happened. The only possible conclusion to be drawn is that the corpuscles suck up water until they burst. Test, finally, the effect of a stronger solution; the corpuscles will shrink. Similar 


\section{8

experiments can be made with the various other kinds of cells making up the bodies of animals, but they require rather more indirect methods. It has been found that the cells of warm-blooded animals remain of a normal size in solutions of cane sugar only when it is about Io per cent., the exact strength differing slightly in the various species. The cells of the frog or fish require a solution of less strength.

What is the explanation of this behaviour?

Suppose that we have a small hollow ball made of an elastic material, which has minute pores in it large enough to allow the molecules of water to pass through, but too small for those of cane sugar to pass. This is filled with a ro per cent. solution of sugar, and immersed in water. It would swell up rapidly, and ultimately burst. The fact which has to be explained is the rushing in of water molecules at a greater rate than they escape, although the membrane is completely permeable to them in both directions. It is somehow due to the presence of cane sugar molecules on the inside of the membrane and their absence on the outside, because this is the only difference. But how? We call to mind the fact that molecules have an actual size and that, in a cane sugar solution, a part of the space is taken up by the solute and, therefore, there are fewer water molecules than in an equal volume of water. Giving our attention next to a particular area of the membrane, we realise that on the outside the whole space is bombarded by water molecules, so that wherever there is a pore, a water molecule can get through. On the inside, a number of these pores will be hit by sugar molecules, which cannot get through. As concerns those hit from the inside and outside by water molecules, as many will pass in a given time in both directions, since the space is merely a part of the general mass of water. But where the sugar molecules hit, no water passes outwards, while there is no hindrance to its passing inwards. The amount that enters is, therefore, proportional to the number of sugar molecules in a given volume of the solution.

If we immerse the ball in a solution of cane sugar of the same strength as that inside it, the number of pores hit by sugar molecules is the same on both sides, so that there is the same limited opportunity for water to pass inwards and outwards, and no change takes place in the quantity of water within. If we place the ball in a solution of half the strength of that inside it, what will happen? Water will enter, because there are more pores free on the outside than on the inside. But, as the water enters, the solution becomes diluted. The ball will expand until its volume has become double that which it first possessed; since, then, the solution within will have become of the same strength as the outer solution, supposing that we had a large volume of solution outside, so that the water 
lost by going into the ball made no perceptible difference in the concentration of this solution. Now, imagine the ball placed in a solution of twice the strength of that within it. The opposite process will take place. Water will pass outwards until the strength of the solution inside has risen to that of the solution outside. The ball will shrink to half its size. We see, then, that such a system behaves exactly like the living cell. But, it may be said. the red blood corpuscles do not contain a solution of cane sugar, True, but the above considerations require only that whatever molecules there are in the solute should be unable to pass through the membrane, no matter what may be the chemical nature of these molecules. The effect is simply proportional to their number in a given volume; in other words, to the molecular concentration of the solution.

The movements of water from one side of a membrane to the other side, when caused by difference of molecular concentration, are known as "osmosis." A membrane which is permeable to the solvent, but impermeable to any particular solute, is called "semiperineable" as regards that solute. The last name is not very descriptive, but is used in the sense indicated. An "impermeable" membrane would be one which does not permit either water or solute to pass through, such as one made of glass would be.

We must next devote a little time to the conception of equimolecular solutions. It is obvious that for chemical operations it is a great convenience to have solutions of which equal volumes contain a known relative number of molecules. For example, suppose that we want to precipitate a solution of sodium chloride by one of silver nitrate. If the solutions are of equimolecular strength, all that we have to do is to take equal volumes, without the necessity of trial; and if we find that a known volume of the silver nitrate solution is just able to precipitate a particular volume of the sodium chloride solution, we know that these volumes contain an equal number of molecules of the reagents. In practice, the most useful concentrations to take are those in which one litre contains the molecular weight of the solute expressed in grams, or solutions of simple relation to these. The molecular weight of a substance expressed in grams is called a "mol," and hence solutions containing one mol in the litre are "molar." Since the molecular weight of cane sugar is 342 , a solution containing $342 \mathrm{gm}$. in a litre is a molar solution. A molar solution of sodium chloride contains $58.5 \mathrm{gm}$. in the litre, and so on.

The solution of cane sugar which we have been using contains $100 \mathrm{gm}$. in the litre, and is, therefore, $100 / 342$, or almost exactly 0.3 molar. If the red corpuscles behave as osmotic systems, therefore, a solution of glucose of the same molecular concentration 
(o.3 m.) as Io per cent. cane sugar should preserve their normal volume. Such a solution has a concentration of 5.4 per cent., since the molecular weight of glucose is 180 , and $180 \times 0.3=54 \mathrm{gm}$. in the litre. If we try the effect of such a solution we shall find it to be equivalent to Io per cent. cane sugar. There are many organic substances which can equally replace cane sugar in the same molecular concentration. We may say, then, that the molecular concentration of the red blood corpuscles, so far as concerns those substances to which their membrane is semi-permeable, is 0.3 molar.

But there are others, a solution of urea, for example, which behave apparently just as water does. Is this because the membrane is permeable to urea, as it is to water? Let us consider what would happen in such a case. For a moment, the number of molecules in equal areas on both sides of the membrane is not the same, but in a very short time urea molecules pass through the membrane, and rapidly become equal in number on both sides, so that there is no longer any difference, as far as urea molecules go, and there is nothing to oppose the inflow of water caused by those molecules which cannot pass through.

We see how we can utilise the changes in volume of cells to find out whether or not their membranes are permeable to various solutes, remembering, of course, that these solutes must not cause injury to the cell membrane. It may also be pointed out that there is no satisfactory explanation of this behaviour of cells to the molecular concentration of solutions, and not to other properties, other than that they have a membrane around them semi-permeable as regards the particular solute in question.

As mentioned before, other cells may be used, and a method with plant cells, known as that of "plasmolysis," has played a large part in the investigation of the phenomena. In this method, plant cells containing in a large vacuole inside the protoplasm a coloured solution, "cell sap," are subjected to the action of different solutions. The protoplasm forming a coating inside the cell wall has a membrane of similar semi-permeable nature to that of the blood corpuscles. If a solution of a higher molecular concentration than that of the cell sap be applied, water will escape through the protoplasm, and a space will be formed between it and the cell wall, visible owing to the coloured fluid in the protoplasmic bag. The experiment may be tried with the staminal hairs of Tradescantia (E., p. I 7 I). By testing various strengths of cane sugar solutions, one will be found which is only just sufficient to cause perceptible plasmolysis. The solution of equimolecular concentration to that of the cell is, therefore, a little below this. 


\section{Osmotic Pressure}

Hitherto, we have considered the effect of the entrance of water in producing a swelling of cells. Suppose that they cannot swell, as is the case with the cells of the higher plants, encased in a cellulose box. What will happen? We may imagine that the membrane of our original ball is rigid and incapable of being stretched, and that we have attached a vertical tube to it, so that the water which enters in may find an outlet. If Io per cent. cane sugar be inside and water outside, we shall see that the solution riscs rapidly in the vertical tube, and finally runs over the top. A pressure is evidently produced by the inflow of water, and this pressure must be greater than that of the column of liquid of the height of the tube. This would, indeed, be expected when we call to mind that the molecules of water get in from the outside in virtue of their kinetic energy. The amount of energy inside the ball is clearly greater than before the extra molecules of water had entered. The increase of pressure, which shows itself by raising the column of liquid in the tube, is what is called the "osinotic pressure" of the solution. It is difficult to make a simple experiment to show this fact, because, although artificial membranes can be made which are semi-permeable as regards cane sugar, it is not an easy matter. But there are some organic substances whose molecules are large enough not to pass through the pores of parchment paper, which are much larger than those of the cell membrane. The experiment may be tried with gum arabic, or with the protein of milk, called caseinogen (E., p. I I I).

We must next get an idea of how great osmotic pressure is. Returning to our membrane impermeable to cane sugar, let us try a much more dilute solution, and instead of allowing it to raise a column of itself in a tube, let it raise the heavier mercury, as can easily be done by connecting the ball to a mercury gauge or " manometer." Pressures of moderate degree are usually expressed in millimeters of mercury, $760 \mathrm{~mm}$. being the pressure of the atmosphere. Taking a I per cent. solution, that is, $0.03 \mathrm{~m}$., we should find that the mercury rose to a height of $5 \mathrm{II} \mathrm{mm}$., and if we took other concentrations, we should find that the pressure was very nearly in proportion to the concentration, so that we may say that the osmotic pressure of the red blood corpuscles and the contents of other animal cells is about $5, \mathrm{I} 10 \mathrm{~mm}$. of mercury, or 6.7 atmospheres. The osmotic pressure of cane sugar solutions has been very accurately measured, and it has been found that the volume taken up by the molecules and other connected phenomena have to be taken account of. They naturally play a much larger part when the solutions are concentrated.

The osmotic pressure of the cell contents is a high one, even 
in the ordinary animal cells. In certain plant cells it is higher still, and may amount to more than eleven atmospheres. It may be asked, why do such cells escape being burst? In the plant cell there is a rigid case around the protoplasm, so that the osmotic pressure makes the cell very stiff (turgor), thus preserving the form and uprightness of even the fragile stalks of plants. If the osmotic properties of the cell are destroyed (E., p. I7I), the rigidity of the structure disappears and the stalk collapses. In the case of the animal cell, which is devoid of such a protection, the liquid in which it lies has the same osmotic pressure as itself, is "isotonic," so that the pressure on both sides of the membrane is the same. When the outer fluid has a lower osmotic pressure (hypotonic), the cells swell or burst. If the plant cell is surrounded by a liquid of the same osmotic pressure as itself, its internal pressure is compensated and the turgor disappears.

We may next take a further step. We have seen that the osmotic pressure is proportional to the number of molecules in a given volume and, as the student is well aware, so is the pressure of a gas. What we have called a molar solution contains one gram molecule in a litre. A gas at atmospheric pressure contains one gram molecule in 22.4 litres. Therefore, if we want to have one gram molecule in one litre of a gas, we must compress it, so that 22.4 litres become one litre. This requires, by Boyle's law, a pressure of 22.4 atmospheres. If we want only $0.3 \mathrm{gm}$. molecule in a litre we require a pressure of only $22.4 \times 0.3=6.7$ atmospheres, identical with the osmotic pressure of a solution of the same molar strength. As mentioned above, however, when accurate measurements of osmotic pressures of solutions are made, it is found that, as would be expected with liquids, we have to make allowance for the space occupied by the molecules in a more important degree than in gases, although it has to be done in this case also, as the reader is probably aware, from his study of the Van der Waals' "equation of state"-a necessary modification of the simple Boyle's law of simple, direct relation between pressure and volume. We see, nevertheless, that the pressure of a gas and the osmotic pressure of a solution are fundamentally the same, and depend on the molecular concentration.

The dissolving of a substance in a solvent, as is well known, raises the boiling point and lowers the freezing point of this solvent. The effect is again found to be proportional to the molecular concentration, and can therefore be used to measure the latter. The boiling point is only of limited application in physiology, since changes occur in the solutions with which we have to deal when the temperature is raised much above that of warm-blooded animals. The depression of the freezing point of a 
watery solution (called $\Delta$ ), on the other hand, is frequently made use of (E., p. I 72), since direct measurements of osmotic pressure are difficult. The vapour pressure is also used for the same purpose (P., p. I 54). The fact that the vapour pressure of a solution is lower than that of the solvent can be foreseen from consideration of the energetics of the process. Imagine two vessels in an enclosed space, one containing water, the other a sugar solution. The pressure must be lower over the latter in order that the osmotic energy of the whole system may be lowered by distillation of water to dilute the solution. The reason why the vapour pressure is less is of the same nature as that discussed in the preceding pages. Air may be regarded as a semi-permeable membrane to a non-volatile solute, since it is permeable to water vapour, not to the solute, which has no vapour. A greater part of the surface of the water is occupied by molecules escaping to the air than in the case of the solution, where a part of it is occupied by the molecules of the solute.

We have spent much time on the question of osmotic pressure, because it is a difficult one; but clear ideas upon it are of great importance.

\section{Electrolytic Dissociation}

Pursuing our investigations on the osmotic pressure of the red blood corpuscles, we shall find that we are led to another very important characteristic of certain substances in solution in water. When isotonic solutions of various materials were tested on plant cells and blood corpuscles, it was found that some of them, although of equal osmotic pressure, were lower in molecular concentration than sugar. Thus, sodium chloride (E., p. I 70 ), if taken in 0.3 molar strength, was too strong and caused the cells to shrink. The correct value was found to be 0.9 per cent., or 0.154 molar; that is, a little more than half its expected value. In other words, these particular substances behaved as if they were split up, or "dissociated," into a larger number of smaller molecules, each of them acting as a separate molecule.

But what can these smaller parts be? They cannot be ordinary sodium and chlorine, because free sodium immediately reacts violently with water, forming caustic soda, and if there were free chlorine in a solution of sodium chloride, it would easily be detected. On further examination, it was noticed that all of these anomalous substances were such as had been found to conduct electricity when in solution; they were salts, acids, or bases. Those that behaved normally were organic compounds and non-conductors.

When an electrical current is passed through a solution of 
sodium chloride by means of two carbon plates immersed in the solution, the current enters by one plate and leaves by the other. Faraday called the two plates "electrodes," that by which the current enters being the "anode," that by which it leaves the "cathode." Since the current flows from the anode to the cathode, the former has the higher potential, or is electro-positive to it. Now we find that chlorine is attracted to the positive pole, and is present around it in solution. Sodium goes to the negative pole, and can be collected if mercury is present to dissolve and remove it ; otherwise, it reacts with water to form the hydroxide. But if the sodium atoms are attracted to the negative pole, it must be because they have an opposite, or positive, charge. Correspondingly, the chlorine atoms must be electro-negative. Since there is every reason to believe that atoms owe their chemical nature to their constitution as electrons, or unit electrical charges, with a positive nucleus, it is clear that if an electron is added or removed, making the atom negative or positive to what it was before, the chemical properties will be altered. A chlorine atom with an extra electron is not the element chlorine, nor is sodium with an electron removed the same thing as the metal sodium. It is only when the sodium and chlorine "ions," as Faraday named them, on account of their movements to the poles, lose their electrical charges by contact with the opposite charges on the poles, that they are converted into the ordinary elements. In Faraday's terminology, the sodium ion is the cation, because it wanders to the cathode; the chlorine ion is anion, because it goes to the anode. If the current is allowed to pass long enough, all the sodium chloride is decomposed by electrolysis, and if we imagine that the last remaining molecule arrives as such at either electrode and is not decomposed until it arrives there, one of its constituent ions must be left free, and must pass through the solution to the other pole. If this were so, it would exist during its passage as an atom with a charge, that is, an ion. Hence we must admit the possibility of the existence of free ions in the solution, and it is natural to suppose that they are present as such before the electrical current is sent through; so that what this current does is to carry those of opposite sign to the appropriate electrode. This is the statement made by the theory of electrolytic dissociation. The ions into which an electrolyte is dissociated in water are the elements of which we are in search. To repeat, a solution of sodium chloride or other electrolyte is already decomposed into its constituent ions, to a greater or less extent, before any electrical current passes through it, and what the current does is merely to attract the oppositely charged ions to the poles of opposite charge to themselves and deprive them of their charges. We see also that the passage of electricity from one pole to the 
other is by means of the charges on the ions, each ion carrying a definite quantity. There are various other reasons for regarding this as the correct account of the phenomena (P., p. 173).

It has been agreed to denote the possession of a positive charge by the addition of a dot to the chemical symbol of an ion, and a negative charge by a dash. Thus the hydrogen ion is $\mathrm{H}$; the chlorine ion is $\mathrm{Cl}^{\prime}$. Ions may possess more than one clarge, according to their valency. Thus, sulphuric acid dissociates into two $\mathrm{H} \cdot$ ions, and one $\mathrm{SO}^{\prime \prime}$ ion, which must have two dashes to satisfy the positive charges of the two hydrogen ions.

Since it is by the agency of water that the dissociation into ions is effected, it is natural to expect that the more water there is in proportion to the solute, the greater will be the degree of dissociation. We have seen that O.I 54 molar sodium chloride is almost completely dissociated into its two ions, since it is equal in osmotic pressure to a 0.3 molar solution of a substance which is not dissociated (E., p. I 7O).

Acids and alkalies, as well as neutral salts, conduct electrical currents excellently; in fact, better than neutral salts. What are the ions here? And why do they conduct better? In the case of acids, we find that hydrogen gas is given off at the cathode, therefore the ion must be hydrogen with a positive charge. In the case of hydrochloric acid, the other ion must be chlorine. All acids are actually found to give hydrogen ions, while the anion varies with the chemical composition of the acid. Alkalies deposit the metallic or similar ion at the cathode. Sodium hydroxide is decomposed into sodium and hydroxyl ions, but since the sodium combines with water giving off hydrogen, it is this gas that actually makes its appearance. At the anode, oxygen is given off, because the $\mathrm{OH}$ when deprived of its charge cannot exist. Two $\mathrm{OH}$ ions unite, forming one molecule of water, and giving off oxygen.

It remains to mention briefly why some electrolytes, as Faraday called those substances which conduct electricity when dissolved in water, are better conductors than others. It has been found by experiments, which cannot be described here, that different ions move to their respective poles at different rates, and according to their dimensions. $\mathrm{H}$ and $\mathrm{OH}$ ions move much faster than any other ions. It is easy to see that the way in which electricity is carried through a solution is by means of the charges carried by moving ions, so that the more rapidly these ions move, the more they carry across in a given time. Hence, substances which dissociate with the production of rapidly moving ions are better conductors than those producing slowly moving ions.

Ions have also the property of attaching molecules of water, which increase their dimensions, and make them move more slowly. 
The number of water molecules attached varies with the different ions. This fact is of importance in connection with the permeability of the cell membrane to them, since inorganic ions become larger than would be expected.

In addition, however, to this cause of difference in conductivity of solutions, there is another in the fact that different substances are split up in very different degrees when dissolved in water. So that, even if their ions move at the same rate, there are fewer of them in the one case than in the other. It must always be kept in mind that those molecules which are not split up into ions take no part in the carriage of electrical currents.

It is not to be understood that all organic compounds are similar to sugar in being non-conductors. Some of them are acids, some are bases, and some are salts. But since they are, as a rule, large and complex molecules as compared with inorganic compounds, they are not such good conductors, although many of them are better conductors than might have been supposed from the dimensions of their molecules. Thus, solutions of congo red are very good conductors, although it is a salt of an organic acid of very large molecular dimensions with sodium.

Strength of Acids and Bases.-It is well known that some acids are very much more powerful chemical reagents than others. Thus, hydrochloric acid in dilute solution dissolves zinc with great rapidity, whereas acetic acid in the same molecular concentration has very little action upon it. Now, if we compare strong acids with weak acids as regards their electrical conductivity, we find that the former are much better conductors than the latter. This might be due either to their being more dissociated, or to the rate of migration of their ions being greater. We can decide this question by diluting (E., p. I75). Suppose that we take hydrochloric acid and acetic acid, each in one-tenth molar concentration. The former is a much better conductor than the latter. Next, dilute each to ten times its volume. We find that the conductivity of the hydrochloric acid is reduced almost exactly to one-tenth. This means that practically no further dissociation has occurred; or, in other words, that it was at first almost completely dissociated. On the other hand, the conductivity of the acetic acid is much greater than one-tenth, hence it must have become more dissociated, since the original ions would only account for a diminution to onetenth. By further dilution, we can make the conductivities of the two acids approach one another nearer and nearer.

It is clear that these considerations suggest to us a method of expressing the "acidity" of a solution in a numerical manner, a fact of great convenience and importance. We have merely to give the molecular concentration in ions; and, since it is only the 
hydrogen ion which is common to all acids, and is responsible for their characteristic acidic properties, such as taste, and so on, we always speak of the "hydrogen-ion concentration." We shall find later that physiological phenomena are extremely sensitive to the precise value of this property of the medium in which they take place, and that there are means taken to maintain it at its most appropriate value.

Similar considerations may be applied to the case of alkaline solutions, and their alkalinity may be expressed in terms of concentration of $\mathrm{OH}$ ions. But, since the product of the $\mathrm{H}$ and $\mathrm{OH}$ ionic concentrations in all solutions is the same (P., p. 197), it is best, for the sake of uniformity, to give the "reaction" of all solutions in terms of $\mathrm{H}$-ion concentration, from which the $\mathrm{OH}$-ion concentration can be easily calculated. The reaction of distilled water being taken as the point of neutrality, those solutions whose $\mathrm{H}$-ion concentration is greater than this are acid, those below it are alkaline (P., p. I 84).

Indicators.-The question next arises as to how this hydrogenion concentration is to be estimated. The most direct method is by the use of the hydrogen electrode, in which a battery is fitted up whose electrodes consist of hydrogen. The electro-motive force in such a case is proportional to the concentration in $\mathrm{H}$ ions of the solutions in contact with the electrodes, and can be measured in the usual way (P., p. 190). But although this method is the most accurate in cases where it can be used, in the physiological solutions of most interest to us its application requires somewhat complicated procedures if correct values are to be obtained. A case in point is that of the blood. For this reason the more indirect methods are, as a rule, more useful. Of these methods, the use of what are known as "indicators" is the simplest. There are many coloured chemical compounds which have a different colour, according to the $\mathrm{H}$-ion concentration of their solutions. There is some dispute as to how this change of colour is related to the chemical changes in the indicator, but this does not concern us here. In general, the range is small, so that above a certain concentration there is no further change in colour, nor is there below a certain concentration. The particular concentration in hydrogen-ions, at which the more or less sudden change in colour takes place, is not the same with the different indicators (E., p. 175); so that it is posssible, by taking an appropriate series, to obtain the $\mathrm{H}$-ion concentration of a given solution with some degree of accuracy (P., p. 189). In most cases of interest to us, the $\mathrm{H}$-ion concentration is not far distant from that of distilled water, and in such cases the dye known as "neutral red" is very useful, since it shows a series of changes, from crimson through red and orange to yellow, in this 
region. Moreover, unlike some other indicators, it is not particularly sensitive to the presence of salts or proteins in the solution. Thus, the H-ion concentration at which a change of tint occurs is practically the same in their presence or absence, so long as they are not in great excess.

That it is the concentration of hydrogen-ion that an indicator really gives information about is well seen by taking a strong solution of hydrochloric acid and diluting it with water. The dye known as "crystal violet" will be found to show a series of definite changes, although nothing has been done except to decrease the concentration. That it is the hydrogen-ion, and not the anion, may be seen by taking a different acid, say sulphuric, when we find the same series of changes (E., p. I75).

The student may notice that this use of indicators differs somewhat from the usual one of determining the amount of total acid present by "titrating" it with a standard solution of alkali, or vice versâ. In such cases, as is seen in practice, the degree of dissociation of the acid does not play any part. Molar solutions of hydrochloric and of acetic acids require the same amount of caustic soda to neutralise them to an indicator. How is this to be explained if the concentration of $\mathrm{H}$ ions is so much greater in the former case? We have only to remember that, as each successive portion of $\mathrm{H}$ ions is combined with $\mathrm{OH}$ ions from the alkali to make water, the remaining part of the acid, becoming less and less concentrated, continues to become more and more dissociated, until the whole of it, whatever the original degree of dissociation, has passed through the ionised state, and the $\mathrm{H}$ ions have been neutralised by $\mathrm{OH}$ ions.

\section{The Electrical Resistance of Living Cells}

Since the electrical current can only pass through solutions of electrolytes by virtue of its carriage in charges on moving ions, it is obvious that the conducting capacity of a solution depends on the width of the channel between the electrodes, as well as on its length. If, therefore, part of this channel is filled up with some non-conductor, such as grains of sand, there must be an obstruction to the passage of a current. Further, if the cell membrane is impermeable to the ions of a solution in which the cells are immersed, these cells must behave simply as inert bodies, blocking the passage of a current - in fact, as if they were grains of sand. This is found by experiment to be the case, and has been used to determine the number of blood corpuscles in a given volume of blood (E., p. 1/5). If the cells are killed, the membrane becomes permeable, and the conductivity rises, because the cells now admit of the ions of the 
solution passing through them with very little resistance. This fact may be regarded as further evidence of the presence of a semipermeable membrane on the surface of the cell: But, on the other hand, it has been objected that the inorganic salts, shown by chemical analysis to be present in the cell, might be combined in a non-dissociable form with the organic constituents, or proteins, of the cell. There are certain methods, which would require more space to describe than can be allowed here, which show that there are free electrolytes inside the cell (P., p. I23). But, apart from this, an indirect proof can be given on the basis of the osmotic pressure of the cell contents, a proof which is instructive in itself. We have seen that the esmotic pressure is that of a 0.3 molar solution. The smallest molecular weight met with amongst proteins is over 3,000 ; haemoglobin has one of 12,000 . Assuming that it is 3,000 , a 0.3 molar solution must contain 90 per cent. of the solute, an impossible amount, since we know that only 20 per cent., at the most, of the cell contents is solid matter. The cell membrane must be impermeable to solutes of small molecular weight.

\section{Changes in Permeability during Life}

The consideration of the preceding paragraph leads us to a brief statement of what evidence there is with regard to such changes.

It has been pointed out above that the cell membrane cannot always be semi-permeable as regards food materials-sugar, for example-when the supply comes to it from the outside, as in the higher animals. We have also seen reason to regard the cell membrane itself as a local concentration of constituents of the cell and of the surrounding medium. Its properties naturally depend on the changes in the cell especially. Hence it is not surprising to find that, in states of activity of the cell, the membrane becomes permeable to substances to which it was previously impermeable. There are not many cases in which, as yet, direct evidence of this has been obtained (P., p. I24). Electrical stimulation of certain contractile cells causes them to lose the pigment which is normally kept within. Again, supposing that the natural electrical resistance of the cells is mainly due to the impermeability of their membranes to the ions of solutions in which they are immersed, it will be clear that this resistance must decrease if the membrane becomes more permeable. Such effects have been detected in muscle in contraction, in the process of fertilisation of egg-cells, and so on (P., p. I4I). We shall see later how this change of permeability to ions explains the electrical phenomena which are frequently to be detected when cells enter into activity. 


\section{The Colloidal State}

We are now in a position to understand more about the particles which were revealed in protoplasm by our special optical methods.

Suppose that we imagine a small piece of gold immersed in water, and by some means gradually divided up into smaller and smaller fragments. Ultimately we shall arrive at the atoms, beyond which we cannot proceed without altering the chemical properties of the substance. But, before this state is reached, we should find that the particles were small enough to be kept in suspension by Brownian movement, and that the preparation would show some new properties. It would appear clear but coloured, and might be taken to be homogeneous unless a bright beam of light were sent through it. When this is done the existence of fine particles of gold is made manifest. Although such solutions of gold, which are said to be "colloidal," could only be prepared with great difficulty by simple mechanical disintegration, they can be made easily by chemical decomposition of solutions of salts of gold. The action of a reducing agent is to split up the salt, so that metallic gold is obtained in a very finely-divided state (E., p. I 76).

Similar solutions can be made by appropriate treatment of various substances usually regarded as insoluble. The gamboge, already used, is one of these. So is the suspension of carbon particles known as "Indian Ink." Such are called "suspensoids," and consist of a solid phase suspended in a liquid phase. Since the solid phase is completely surrounded by the liquid one, it is the "internal phase," and may be compared to a number of islets surrounded by the sea. But it is clear that the same constituents might be arranged differently, similar to a number of small lakes surrounded by land, such as might happen if the islands grew until they touched one another. Here the solid phase would be external and the water internal. The whole system would be solid, instead of liquid.

Further, the constituents of a colloidal solution may be two liquids which do not mix with one another. These systems are "emulsions," or, when their internal phase is very finely divided, "emulsoids." A good example is cream, where the internal phase consists of oil globules, the external phase is a watery solution. When made into butter, a redistribution of phases occurs by the oil globules uniting together; the fat becomes external, the watery solution in droplets surrounded by it. The meaning of the terms sometimes used will be plain. The internal phase is the "dispersed" one, the external phase is the "continuous" one. It is very likely that changes in distribution of phases plays an important part in the mechanics of the cell and of its membrane. 
But an emulsoid system may also be formed by dispersion of a solid in a watery phase, provided that this solid is one that soaks up water by the process known as "imbibition." A wellknown case is that of gelatin (E., p. I77). Here the redistribution of phases takes place merely on warming and cooling. A jelly consists of droplets of a very dilute solution of gelatin encased in chambers of the solid gelatin holding water in its substance by imbibition. On warming, the more solid phase becomes internal, particles surrounded by watery solution. Hence the system, as a whole, becomes liquid. What the nature of imbibition is, is not completely known. There is evidence that it is essentially an adsorption of water by the surfaces of constituent elements of the solid, owing to certain physical peculiarities of these surfaces; but the precise interpretation clearly depends on what these elements are.

Whatever may be the nature of imbibition, a fact of importance in the physiological behaviour of emulsoids is that the amount of water present may vary in its distribution between the two phases. The change is produced especially by electrolytes (E., p. I77), not in virtue of their electrical charges, but owing to the effect they have on the properties of water (P., pp. 96, 97). The importance of being able to extract water from a system in which chemical reactions are taking place will become more evident when we study the actions of enzymes.

Remembering that a colloidal solution consists merely of a substance very finely divided and dispersed in a liquid, we see at once that the properties that distinguish it from those of a system consisting of the same amount of material in a single lump immersed in the liquid depend on the enormous extent of boundary surfaces between liquid and solid phases, so that they may be regarded as only differing in degree; but there is a very great difference in degree. The properties are, therefore, those which manifest themselves at such interfaces. These are especially those dependent on surface tension, electrical charges, etc. We expect to find adsorption phenomena in a marked degree, and we shall see, presently, the way in which electrical charges play their part. From this point of view we may note again that we cannot make any hard and fast line of distinction between coarsely heterogeneous and colloidal systems, except in degree. On the other side, it is difficult to say at what stage of subdivision the properties of surface cease and molecular properties begin. As will be seen presently, some molecules are large enough to show the properties of surface when single, but in most cases, and especially in the suspensoid colloids, the particles consist of a large number of molecules. It is generally agreed, however, to call those solutions 
"colloidal," of which the dispersed phase is in large enough particles, be these aggregates or single molecules, not to pass through parchment paper, while at the same time small enough to remain suspended permanently, or for a long time.

When such solutions were first described by Thomas Graham, the colloidal state was thought to be a property of certain substances, such as gelatin or glue only; hence the name (кó $\lambda \lambda \eta$, glue). But we now know that any substance, by appropriate treatment, can be brought into the state. In general, the treatment may be described as reducing the material in question by some means or other to a very fine state of subdivision. In the case of chemical elements or simple compounds of small molecular dimensions, the colloidal particles are aggregates of a large number of separate molecules, but it is obvious that a single molecule, if large enough, may exhibit colloidal properties. Such is the case with some dyes, as congo red; and with the proteins, of great physiological importance, whose nature we shall learn in the next chapter. We must remember that the visibility of the particles depends on the brilliancy of the illumination, and on the fact whether they differ much in refractive power from the liquid in which they float. There are, indeed, some substances which we know to be in colloidal solution, because they do not pass a parchment paper membrane, but which require a very powerful illumination to show the presence of particles. Some only show a diffuse beam of light when observed under the best conditions yet possible; they have not been actually resolved into separate particles. On the other hand, if the illumination is sufficiently powerful, even simple molecules may show a beam of scattered light; in fact the blue of the sky is such light scattered by the molecules of the gases of the atmosphere.

We must now direct some attention to the properties which belong to colloids in consequence of their enormous development of surface. First of all; there are certain properties due to the presence of surface tension, or rather of surface energy. Since the larger the number of particles into which a given mass is divided, the greater the total area of surface, there will always be a tendency for these particles to aggregate together again into larger masses, for by doing so there will be a diminution of free surface energy. This tendency is opposed by the continual Brownian movement, and we can also decrease it by diminishing the intensity factor of surface energy, that is, the surface tension, by the addition of some substance which lowers the surface tension at the interface. Solutes in general do this, as we have seen, but there are some which have a very marked effect of this kind. Such are the higher alcohols, bile salts, fatty substances, saponin, etc. (E., p. I77). But 
there is another phenomenon which takes part in the maintaining of the particles in suspension, and is also of importance in other ways. If we place a colloidal solution between electrodes, connected to a battery so that there is a fairly high difference of potential between them, we shall find, in nearly all cases, that the colloidal particles are carried either to the positive or to the negative pole, and deposited there. In the former case, they must have a negative charge; in the latter, a positive one (E., p. I77).

We may ask, what is the effect of this charge on the surface tension? Remembering that charges of the same sign repel each other, we may look upon the surface of each particle as made up of areas charged with the same sign; the parts of the surface mutually repel one another, so that the surface tends to increase its area. This is in opposition to the direction of the ordinary surface tension, due to internal pressure, and the result is a favourable one on the state of suspension of the colloid. The mutual repulsion of the particles themselves also plays a part in keeping them from aggregation and deposition.

How is the presence of this electrical charge on the surface of substances in contact with water to be accounted for? There is no doubt that, in the majority of cases, it is due to electrolytic dissociation of the material at the surface of the particle itself. This takes place in two somewhat different ways, according to the dimensions of the molecule of the chemical compound concerned, giving rise, on the one hand, to what have been called "electrolytic colloids," or, on the other hand, to "electrolytically dissociated colloids." As an instance of the former, in which the particles consist of a large number of small molecules aggregated together, let us take silicic acid in the colloidal state. This substance is usually regarded as being insoluble in water, but it is not absolutely so, as indeed no substance is. When in solution, silicic acid, like all other acids, dissociates into hydrogen-ions, which are freely soluble, and anions of silicon oxide, which are practically insoluble. Consider now the state of affairs at the surface of a particle of silicic acid in water. The molecules of the surface layer are electrolytically dissociated. The hydrogen-ions pass into the water, leaving behind on the surface the insoluble silicic anions. These latter possess negative charges, so that the particle, as a whole, will have a negative charge consisting of the sum of the charges of the anions on its surface. The particle becomes a kind of large composite ion, and may be called a "colloidal ion"; but it must be remembered that such ions vary greatly in the number of molecules they contain, so that the charge is not composed of a definite number of electrons, like that of the true ion is. Similar considerations apply to particles of 
basic substances, such as those of aluminium hydroxide. In these cases the soluble ions which go away into the water are $\mathrm{OH}$ ions, so that the particle is left with a positive charge.

Turning now to those substances which are present in solution in single molecules, but are colloidal on account of one of the ions being of large dimensions and insoluble, it is clear that these ions will have charges of a definite number of electrons, according to their valency. Otherwise, their behaviour is the same as that of the previous kind. To distinguish them, however, they may be called "electrolytically dissociated colloids." They are met with, especially amongst complex organic electrolytes; many of the aniline dyes and the proteins are examples. The behaviour of the latter is of special interest, and will be described in the next chapter.

There is one point about the electrical state of such systems as those just referred to that must not be left unmentioned. When the diffusible ion goes into solution in the water surrounding the particles it is endowed with kinetic energy, of course, as all the other molecules of the liquid. In virtue of this, it naturally tends to wander away into the solution. But this is prevented by the powerful electrical attraction exerted by.the oppositely charged solid particle. The soluble ion can only go so far as the balance between its kinetic energy and the electrostatic attraction permits it. A number of them form, thus, a sheath or layer at a very short distance away from the particle. Such an arrangement is known as the "Helmholtz double layer," and we shall have occasion to return to it again later.

In certain cases where the dispersed phase shows on investigation that it has an electrical charge, it is not an easy matter to explain it by electrolytic dissociation, although this may ultimately turn out to be the case. Droplets of paraffin oil in water are negatively charged. It has been suggested that this charge has an origin similar to that of frictional electricity.

There is, again, a further cause of an electric charge on inert particles in solutions of electrolytes. If particles of carbon are suspended in water, surface tension is present at their contact surfaces with the liquid. By the deposition of ions on this surface, adsorption, in fact, the surface energy can be lowered. This may be either in the mechanical way, or by imparting an electric charge. It is a matter of experiment that if acid is present in the liquid phase, hydrogen ions are deposited on the surface, giving it a positive charge. If alkali is present, the surface becomes negative by deposition of $\mathrm{OH}$ ions. There must be some reason why the $\mathrm{H}$ and $\mathrm{OH}$ ions are deposited in preference to these of opposite charge which are always present. It may be that the greater 
velocity of the former ions is the cause, but the matter is not quite cleared up.

Precipitation by Electrolytes.-The neutralisation of the electric charge on colloidal particles will have the effect of throwing them down from suspension, since the removal of the charge acts both by increasing the surface tension and by abolishing the mutual repulsion of the particles. The addition of an electrolyte is an effective way of doing this. Suppose that we add to a colloidal solution of arsenious sulphide, whose particles have a negative charge, some sodium chloride in solution. There are now present sodium ions, with a positive charge, and chlorine ions, negatively charged. The sodium ions neutralise the negative charge of the particles by being deposited on their surfaces, the colloid is precipitated, carrying with it the ions required to neutralise the charges on the particles. Since it nceds several univalent ions to neutralise the charge on each particle, it is clear that many, ions have to be met with by each particle before sufficient opposite charge has been obtained. Bivalent or plurivalent ions afford two or more electrons at each encounter, so that they are much more effective, as would be expected by the law of chances (E., p. 178). When we have an electro-positive colloid, it is the anions of the added electrolyte that are the active ones. We see that a decrease of free energy occurs by such abolition of charge, whereas if ions of the same sign as the surface were deposited on it, a gain of free energy would result.

Emulsoid colloids are, as a rule, much less sensitive than suspensoids to the action of electrolytes. But it is only a matter of degree (P., p. 92). If we call to mind that the two phases of which the former consist differ only in the amount of water contained, it will be understood that the forces at the interface of contact, whose magnitude depends on the difference in nature of the two phases, must be less than when the two phases are altogether different in chemical composition.

This is an appropriate place to remind the student that the various physical properties to which, for the time, our attention is being directed, depend on the chemical nature of the substances concerned. While we discuss the properties which belong to certain constituents of the cell on account of their being in the colloidal state, we must not forget that they also react chemically with other constituents and with substances coming from the outside. Substances in the colloidal state, however, do not so readily enter into chemical reaction with other substances, since it is only the surface of the matter of which they are composed that comes into relation with other reagents. On the other hand, the physical properties of the surface can be brought into play very 
rapidly, a point of some importance in connection with the interpretation of certain physiological phenomena, such as that of muscular contraction (E., p. I79).

When electrolytes are added to colloidal solutions, if more is added than necessary to neutralise the charges, the particles may have conferred upon them a charge of the opposite sign to their original one and be re-suspended. It is somewhat difficult to give a satisfactory explanation of this fact. The probable reason is that the excess ions are adsorbed, owing to their effect on the mechanical surface tension. If an ion is adsorbed owing to an effect of this kind, independent of the sign of its charge, the surface must obtain a charge of the sign of that of the ion in question (E., p. I79).

The addition of a colloid of opposite electrical sign to another colloid has the effect of precipitating both (E., p. I79). Excess of either causes re-suspension, owing to the excess charge of one sign or the other.

The precipitate in this last case is evidently composed of both colloids, although not in chemical combination. It is a representative of a large class of substances, sometimes called "adsorption compounds." The components of these are present in no relation to chemical combining proportions, but to certain physical properties, which, it may be pointed out, although less simple to determine in any particular case, follow laws as definite as the purely chemical ones. Much confusion of thought would be avoided if the expression "chemical combination," or even "combination," were strictly confined to those cases where the chemical properties of the atoms or molecules are changed-where the internal structure and energy of the atom is altered. The name "adsorption compound" is not to be recommended ; a better name is "colloidal complex." Such complexes may be formed between colloids and crystalloids, as when charcoal takes up iodine, as well as between two or more colloids (P., p. 64). There are other cases, such as those of mixed crystals and that of the water of crystallisation, where physical, rather than chemical forces appear tu be concerned.

From the general properties of contact surfaces, as outlined above, we see that complexes between colloids and substances that lower surface energy are very apt to occur. They often cause difficulty in the separation and purification of the compounds present in cells and secretions, as will be more obvious later. Many errors in interpretation have been made on this account.

\section{Electrical Adsorption and Histological Staining}

The dyes used for the purpose of making evident various con- 
stituents in cells are nearly always neutral salts, but in the one set the coloured ion is a complex organic acid combined with an inorganic cation, usually sodium ; in the other set, the cation is the coloured one, and is combined with an inorganic acid, usually hydrochloric or sulphuric. The former set is often called that of the "acidic" dyes, the latter, "basic" dyes, but such names are clearly misleading, in that they suggest that the dyes themselves have the properties of acids or bases. It was supposed at one time that chemical combination occurred between particular constituents of the cell and dyes of a definite chemical composition, so that the staining of some particular structure indicated that it had some particular chemical composition. Although this seems to be the case in some rare instances, further investigation has shown that a great variety of physical conditions also play a part, and that a conclusion of the kind referred to cannot be drawn without other evidence. Some points that are instructive may be mentioned here. The ordinary form of adsorption must play a part, but there are also those phenomena in which electrical forces come into action, and sometimes in a rather complex fashion. Most of the surfaces in cells have negative charges, and in order to see how they behave to various dyes, some experiments with filter paper should be made; since this has a negative charge in water, the conditions in general can be readily controlled (E., p. 179). The results obtained apply, naturally, with the appropriate change of sign, also to surfaces having a positive charge.

Let us take pure white paper and stain some pieces of it in crystal violet, a "basic" dye, and others in congo red, an "acidic" dye. The former will rapidly become deeply stained, the latter very faintly. The explanation is, no doubt, that in the first case the ion which stains the paper is the electro-positive one, and is attracted ; in the latter case, the coloured ion is electro-negative, and is repelled by the paper. What staining occurs in the case of congo red is the mechanical adsorption due to direct effect on the surface tension. That this is so is shown by the curious fact that the coloured matter deposited in the case of "basic" dyes is the free base, whereas in the other case it is the neutral salt itself. This is the reason why, in our previous experiment with charcoal (E., p. I68), we used acidified alcohol to remove the dye from the surface. Next, add a neutral salt, say sodium chloride, to both the stains, and repeat the above experiments with filter paper. It will be found that congo red stains very deeply, while crystal violet stains less deeply than in the pure state. Why is this? The negative charge on the paper is neutralised, or changed to a positive one, by adsorption of sodium ions from the solution, so that the attractive and repulsive powers of the paper towards the two 
opposite signs of coloured ions are reversed. This is the explanation of the effect of electrolytes in certain histological staining reactions. The phenomena in general are spolen of as "electrical adsorption," a term which also includes the adsorption of the precipitating ion in the ordinary process of precipitation of colloidal solutions by electrolytes.

\section{Hydrolytic Dissociation}

There is a form of dissociation to be met with in salts of weak acids or weak bases which is not of an electrical nature, and, to a certain extent, antagonistic to electrolytic dissociation. This is known as "hydrolysis," or, better, "hydrolytic dissociation," because it is brought about by interaction with the hydrogen and $\mathrm{OH}$ ions of water.

We have already seen that the distinction between weak and strong acids or bases is that the former are only slightly dissociated electrolytically, unless very strongly diluted. If we take a salt of such a weak base, say ammonia, with a strong acid, such as hydrochloric acid, or of a strong base, such as sodium hydroxide, with a weak acid, such as acetic acid, or of a weak base with a weak acid, such a salt as ammonium acetate, and dissolve in water, we find that the solution is not neutral in reaction. There is evidence of the presence of free acid and free base. The reaction will be either acid or alkaline, according to which is the stronger. Ammonium chloride is acid, sodium acetate is alkaline. Further details of the process will be found in the larger works (P., p. 196).

Although the fact has sometimes to be reckoned with, it is not to be supposed that it is usually of any great magnitude, unless both the acid and the base are extremely weak; and in such cases the question naturally arises as to whether we are justified in speaking of their being in combination at all. It is usually not more than I to 2 per cent., and is sometimes absent when it might have been expected to be present.

The nature of the phenomenon may be realised somewhat in the following way. If we take a.solution of sodium acetate and suppose that electrolytic dissociation occurs in the usual way, it would be almost completely dissociated into sodium ions and acetic anions. Now, although the former can exist in high conccntration in water, the latter cannot, since acetic acid is but little dissociated. The acetic anion accordingly combines with hydrogen ions from the water, forming undissociated acetic acid; more hydrogen ions are set free from the water until the normal proportion of dissociated and undissociated acetic acid is present. 
The result is that an excess of $\mathrm{OH}$ ions remains, giving an alkaline reaction to the solution.

The adsorption of the free base of "basic" dyes is connected with the hydrolytic dissociation of these salts, since the base is a weak one. Being insoluble in water, it forms a colloidal solution therein, and, owing to its giving off $\mathrm{OH}$ ions, becomes electropositive and powerfully attracted by a negative surface. Most of the "acidic" dyes are salts of fairly strong acids (sulphonic acids), and are very little, if at all, hydrolysed in solution.

\section{The Nucleus of the Cell}

The presence of a special component in the more highly developed cells has been mentioned. Most of the facts concerning the nature of protoplasm, given in the preceding pages, apply also to the nucleus, but it has functions peculiar to itself. As yet very little can be said about how these are performed. We know that if a cell, such as an amœba, is divided so that one part only retains the nucleus, this part will continue to live, while the other part will, sooner or later, die and disintegrate.

When nucleated cells multiply by subdivision, the nucleus usually undergoes a complicated process of activity, to which further attention will be given in the last chapter. It has been supposed, also, to be concerned with the formation of certain structures which appear in the cell, but it has to be confessed that we are still very much in the dark as to its mode of operation.

\section{Mitochondria}

The same statement of uncertainty must be made with respect to those bodies or granules in the cell protoplasm, to which the above name has been given. They have various shapes, and have been seen in living cells, where they appear to undergo changes in the course of the activity of the cell. They have a special attraction or affinity of some kind for a particular group of dyes, known as the derivatives of di-ethyl-safranin.

The subject matter of the preceding chapter is undoubtedly a difficult one, but a comprehension of it is necessary before we can proceed further with profit. The student is recommended to refer back to it from time to time as he meets with phenomena, which require a knowlege of the particular facts referred to here if they are to be understood. 


\section{FOOD-DIGESTION AND RESPIRATION}

THE first question that occurs to us in this connection is-Why do living beings require to take food, that is, some material from the outer world which supplies something that they are in need of?

When such a creature is actually engaged in making greater the amount of substance in its body, is growing, as we say, it is quite clear that this extra substance must be obtained from outside, and what is taken in this way must contain the correct chemical constituents that are wanted to make up the new body tissues.

Further, even in the adult, when growth has ceased, there is a certain loss of material, due to wear and tear in the process of activity, as well as the growth of some parts, such as hair, which continues to take place. As a motor car uses up tyres, piston rings, bearings, etc., so the cell machinery requires replacement of parts worn out. This is sometimes called maintenance, and has practically the same requirements as growth. But not altogether, since there is evidence that some parts, once constructed, never require replacement, somewhat like the fly-wheel of a petrol motor, which lasts as long as the engine itself, apart from accidents.

The amount of food needed by the adult for the purpose of replacing wear and tear is very small. It might be expected, for example, that the structure of muscle would be worn away to some perceptible degree by vigorous exercise. It is a rather remarkable fact that it has been found impossible to obtain evidence of any loss of the actual muscular structure itself, except after such severe work as to be abnormal. There is more evidence of wear and tear in some peculiar forms of muscular work, as we shall see later. It might, perhaps, be said that in ordinary muscular work the products of the wear and tear are used up again to repair the machine. This may be so, but, as far as the necessity of supply from the outside is concerned, the result is the same. 
Nevertheless, as every one knows, a fairly large amount of food is necessary to the adult, especially if he is doing hard work. The last remark indicates the purpose of this food. In fact, by far the greater part of the food taken, both by the adult and the growing organism, is for the purpose suggested, not for growth or maintenance. To do things, to cause changes, requires energy; and, when energy has been used, it must be replaced if more work is to be done. This is the chief function of food.

We will discuss the two uses of food in turn, taking first that for growth and maintenance.

Since the object of this is to make new substance or to replace what has been lost, it is clearly necessary to know what is the chemical composition of protoplasm, and of the various structures made by it. We may, indeed, to begin with, take the general composition of the organism as a whole. We find that it is composed of organic and inorganic substances (E., p. I8I). The latter are not present in very large amount, but are of some variety, and of great importance. Organic compounds, as the student will not need to be reminded, are the compounds of carbon. Of these there are an enormous number known, and a very large number are produced by living beings. The other chemical elements making up these latter carbon compounds are nitrogen, hydrogen, oxygen, and to a less extent, sulphur and phosphorus. Iron and magnesium are found in two very special compounds, as we shall see presently.

The four elements, oxygen, hydrogen, sulphur, and phosphorus, are obtained in the course of taking as food those substances which are necessary as sources of carbon and nitrogen, since animals cannot build up their structure from elementary carbon. and nitrogen. Plants cannot utilise the former, but a few exceptional micro-organisms can take nitrogen from the atmosphere and form compounds useful for food to higher organisms.

\section{Source of Carbon-the Sugars}

While there is a large variety of organic compounds which serve the animal for this purpose, it has been found that none simpler than the sugar called glucose is of use. Green plants, on the other hand, are able, by making use of the sun's energy, to produce glucose for themselves from the carbon dioxide of the atmosphere. Our study of energetics has shown us that, since carbon dioxide cannot be further burned up, it must be converted into a compound that can be so oxidised, if it is to serve as a source of energy. It must have energy supplied to it for this 
purpose, and the source of this supply is the sun, whose rays are absorbed by the green pigment of those plants which possess it. Other plants, fungi, require sugar or similar substance to be supplied ready made, although a few of them are satisfied with somewhat simpler carbon compounds, so long as these have a higher chemical potential energy than carbon dioxide has.

Before we proceed further it will be useful to remind ourselves of the reasons why carbon forms such an enormous variety of different compounds, and is, therefore, particularly fitted to be the basis of the chemical changes taking place in living organisms. In the first place, owing to its possession of four valencies, it is able to form a great variety of derivatives of any one compound, one valency combining with a group of one kind, another with a different one, and so on. Secondly, the power that carbon atoms have of combining with one another, gives the possibility of great complexity and size of compounds. Thirdly, carbon is able to combine with elements of opposite characters, owing to its position in the middle of the periodic table. Thus, it can combine with hydrogen or oxygen, with nitrogen or chlorine. It can, therefore, be alternately oxidised and reduced, thus acting as a carrier of energy. The reduced compounds give off energy when oxidised or burned, while the oxidised compounds require the addition of energy in order to reduce them. Fourthly, it alters its character according to the groups with which it is combined. Thus, while $\mathrm{NO}_{2}-\mathrm{C}=\mathrm{H}_{2}$ is usually "negative," that is, has special affinity for

elements like hydrogen, $\mathrm{CH}_{3}$ is positive, like hydrogen, and has affinities similar to those which hydrogen has. Fifthly, carbon compounds react slowly, or are comparatively stable. Reactions which proceed of themselves with explosive rate are incompatible with vital phenomena. $\mathrm{H}_{2} \mathrm{SO}_{3}$ (sulphurous acid) is much more reactive than $\mathrm{HCH}_{3} \mathrm{SO}_{3}$ (methyl-sulphonic acid). On the other hand, this same property enables large molecules of high potential energy to be built up, which remain stable when left alone, but decompose with great violence when the powerful shock of a detonator acts upon them.

Returning now to glucose, we note that there is a class of compounds containing carbon atoms and water molecules in an equal number. For this reason they are called carbohydrates. The actual numbers of the carbon atoms vary from one to six, or more. The most important ones, from our present point of view, are those of six atoms. They are the sugars called hexoses. The five carbon sugars, or pentoses, are of frequent occurrence in plants, and form an important, although not large, constituent of certain compounds in the nucleus of the animal or plant cell. It appears, 
however, that the pentoses are formed indirectly from the hexoses.

The simplest "carbohydrate" is naturally that with one carbon atom; what is this? If we try to represent in a formula a compound of $\mathrm{C}$ and $\mathrm{H}_{2} \mathrm{O}$, we find that it must be the following:

$\mathrm{H}$

$\mathrm{H}-\mathrm{C}=\mathrm{O}$, which is known as formaldehyde. Looked at from another point of view, it is an aldehyde group (CHO) combined with $\mathrm{H}$. It is the starting point of a number of hexoses, including glucose, which have the properties of aldehydes, and are hence called "aldoses." Adding further carbons and waters so as to form a chain, they must go between the carbon and one of the hydrogens of formaldehyde, and consist of a series of carbons united to $\mathrm{H}$ on the one side, and to $\mathrm{OH}$ on the other. Thus :-

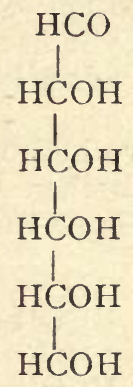

$\mathrm{H}$

Note that there is now an aldehyde group at one end, and an alcohol group at the other end. Aldehydes have powerful reducing properties, taking up oxygen to become acids, so that the CHO group becomes COOH. This latter group is known as "carboxyl," and confers acidic nature on the compounds in which it is present.

Looking at the general formula given above, we see that one or more of the $\mathrm{Hs}$ or $\mathrm{OHs}$ may be changed from one side to the other of the central line. Thus a number of different sugars are possible, many of which are known, although of the aldoses only glucose, galactose (in milk sugar), and mannose (a rare hexose) are of use to the organism. The capacity of dealing with the others is absent. Also, to avoid error, it should be pointed out that there is reason to believe that the simple chain formula, as given, is that produced by the action of reagents, and that the usual state of these sugars is in that of a closed ring by union of the aldehyde carbon to the oxygen of the fourth carbon below, the hydrogen 
of this hydroxyl then becoming transferred to the aldehyde group. Thus:-

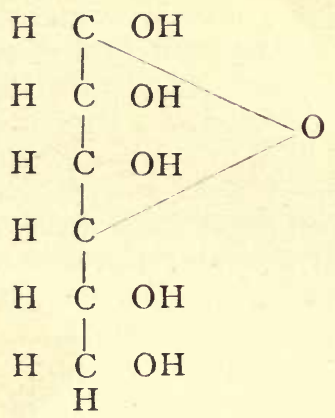

Changes of this kind are common in organic chemistry. If we designate the neighbouring carbon to the aldehyde by the prefix $\alpha$, that one united to the aldehyde in our formula will be $\gamma$, and the compound is a $\gamma$ lactone, being of the nature of an internal anhydride.

Next, if we transfer the two hydrogens from the $a$-carbon to the aldehyde group, we have another kind of sugar which has an alcohol group at both ends and a $\mathrm{CO}$ next to it at one end. Thus :-

$\mathrm{H}$
$\mathrm{HCOH}$
$\mathrm{CO}$
$\mathrm{HCOH}$
$\mathrm{HCOH}$
$\mathrm{HCOH}$
$\mathrm{HCOH}$
$\mathrm{H}$

$\mathrm{CO}$ is the characteristic group of the ketones, and the sugar in question is called a "ketose." It is known as "fructose," and exists, combined with glucose, in cane sugar.

The student should never forget that the representation of chemical compounds in the way that we have done is a conventional diagram of the facts shown by the properties of these compounds with regard to the particular constituent atoms which are united to each other. For one thing, we are compelled to write them on a plane surface, whereas, of course, they are solids, with three dimensions in space. The "bonds," again, are not hooks, or similar rigid attachments, but forces, probably of electrical nature. Further, there is every reason to believe that an element in a particular kind of combination is not the same thing as it is in another kind of combination, or when free, although the change from 
one state to the other may be brought about more or less readily. The energy obtained when a compound of carbon with hydrogen is oxidised to $\mathrm{CO}_{2}$ and $\mathrm{H}_{2} \mathrm{O}$ must arise from changes in the internal structure of the atoms, as already pointed out. We have just seen that $\mathrm{O}=\mathrm{C}-\mathrm{H}$ and $\mathrm{O}=\mathrm{C}-\mathrm{OH}$ have quite different properties, although both contain $\mathrm{O}=\mathrm{C}-$. The carbon is united to $\mathrm{H}$ in the one case, to $\mathrm{OH}$ in the other. The energy content of the former is greater than that of the latter.

\section{Source of Nitrogen - the Amino-Acids}

IVe may now pass on to the consideration of the simplest compound of nitrogen that will serve for animal nutrition, leaving the case of the plant to be dealt with later. The animal organism cannot utilise any compounds of nitrogen simpler. than those known as amino-acids. What is the chemical nature of these substances?

The carboxyl group, $\mathrm{COOH}$, is a characteristic of acids. Although its place can be taken by sulphur or phosphorus derivatives of similar nature, the most important organic acids concerned with the functions of living cells are the carboxylic acids. The free bond of the carboxyl group must, of course, be united with some other molecular group, and the most obvious to begin with is hydrogen; thus we get formic acid, $\mathrm{H}-\mathrm{COOH}$. Many series of compounds of ascending degree of complexity and size are formed by the successive addition of $\mathrm{CH}_{2}$, one of the free bonds of the carbon being used to join on to the original compound, while the other serves to attach further groups. Adding $\mathrm{CH}_{2}$ then to formic acid, we get acetic acid, $\mathrm{H}-\mathrm{CH}_{2}-\mathrm{COOH}$. Continuing the process, we have the numerous straight chain fatty acids: $\mathrm{H}-\left(\mathrm{CH}_{2}\right) n-\mathrm{COOH}$.

One of the commonest and most important compounds of nitrogen is ammonia $\left(\mathrm{NH}_{3}\right)$, so that it is not surprising to find that its derivatives form the basis of the source of nitrogen for living matter. It is joined on to an acid, such as acetic acid, in the form $\mathrm{NH}_{2}-$, taking the place of one of the hydrogen atoms combined with a carbon other than that of the carboxyl :-

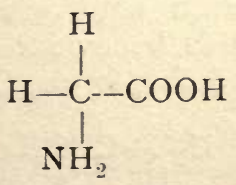

which is amino-acetic acid, or glycine. 
At this point we may note that the only set of amino-acids of use to protoplasm is that in which the $\mathrm{NH}_{2}$ is attached in the a-position as regards the carboxyl. Thus amino-butyric acid might be-

\section{$\mathrm{CH}_{3}-\mathrm{CH}_{2}-\mathrm{CH}\left(\mathrm{NH}_{2}\right)-\mathrm{COOH}$ or $\mathrm{CH}_{3} \mathrm{CH}\left(\mathrm{NH}_{2}\right) \mathrm{CH}_{2} \mathrm{COOH}$}

but it is only the first that is of value. The nitrogenous constituents of the cell structures are all of this $\alpha$-series.

Practically all organic acids-and some of them have very complex structure-can form amino-derivatives. In some compounds we find that another hydrogen atom has been lost from ammonia, and we have the group $\mathrm{NH}=$, the bivalent iminogroup.

When we try to feed an animal on amino-acids only, as source of nitrogen, we find that it can be done if we take a sufficient variety, but that one kind alone is insufficient. There are some particular ones that are necessary, because the animal cannot make them out of the appropriate fatty acid and ammonia as it is able to do in the cases of others. The complex one known as tryptophane, in which a ring containing nitrogen is united with amino-propionic acid, or alanine, is one of these. If we set about making an electric motor, we discover that certain parts of it must be made of substances with definite properties, different from those of other parts. While the wire must be a conductor, the segments of the commutator must be separated from one another by a material which is an insulator, and any conductor would be useless for this purpose. It is possible also that some special chemical groups may be required for the manufacture of substances of importance as regards their action on protoplasmic processes, not as actual components of the machinery. For the lubrication of the bearings of our motor some oil is wanted, and water would not suffice.

With regard to the general properties of amino-acids, it is to be noted that, while the carboxyl group confers those of an acid, the $\mathrm{NH}_{2}$ group is basic. Thus, these acids are what are called "amphoteric," being both acids and bases. But it must be remembered that the acidic and basic properties are potential only. Amino acids in which the two characters are almost balanced, as when there is one acidic and one basic group, are unable to combine with neutral salts, nor even with weak acids or bases. The probable explanation of this behaviour is that such acids exist, even in solution, in a closed ring form. Thus glycine :-

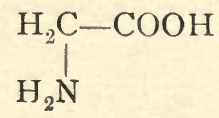


becomes, by formation of an internal salt :-

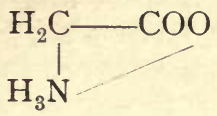

Before they can unite with acids or bases, they must be converted into the hydrolysed form. This can be done by strong acids and bases only, not by weak ones, nor by neutral salts.

There are some amino-acids which possess two carboxyls and one $\mathrm{NH}_{2}$, while others have two basic groups to one acidic group. The former, of course, are much stronger acids than those in which the two functions are nearly balanced, while the latter are strong bases. Both of these classes are good conductors of electricity, whereas the mono-amino-mono-carboxylic acids are scarcely conductors at all, being electrolytically dissociated only to a minute degree.

Although a sufficient variety of amino-acids, as said, suffice as nitrogen supply to an animal, it is not in this separated form that we take them in our food. In fact, if we wanted them so, we should have to make them from the materials which we actually use, and with great difficulty and expense. These materials are the "proteins," of which there are a great variety, differing in the particular aminoacids they contain and in the number of these combined together. This number is always a large one, although there may be several molecules of one kind of acid. Familiar examples are white of egg and the lean of meat.

The way in which amino-acids are combined together is by the union of the amino-group of one acid with the carboxyl group of another, water being eliminated in the way that is so common in organic chemistry. The head of one molecule joins on to the tail of another, as it were:-

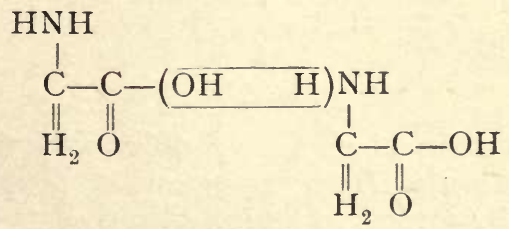

which represents the production of what is called a "dipeptide," namely, glycyl-glycine. The union of - OC- and - NH- to - OC-NH - is known as the "peptide linkage." By continuing the process, more and more acids can be united, forming "polypeptides," and ultimately proteins.

Considering the large dimensions of their molecules, we naturally expect proteins to behave as colloids. They have, in fact, the 
properties of the emulsoids. One of these, as we saw, is that of taking up more or less water, according to surrounding conditions : an important fact. They have also the properties due to the possession of surface, as well as those more definitely due to their chemical composition. Of these we may note that, owing to terminal free $\mathrm{NH}_{2}$ and $\mathrm{COOH}$ groups, as well as those of some amino-acids attached as side branches, they act either as acids or bases towards strong bases and acids respectively.

\section{Optical Activity}

We have seen that, when there are alternatives in chemical compounds of the same general structure, such as the $a$ - and other series of amino-acids, the living organism has been evolved in such a way as to be able to make use of one kind only. This applies to the proteins and to the carbohydrates. Of the eight possible forms of the aldo-hexoses, only three are utilised, namely, glucose, galactose, and mannose.

There is, moreover, in addition to this exclusiveness, a further one to which we must give a little attention.

If we write the formula of methane thus:-<smiles>C</smiles>

we see that the carbon atom is symmetrical on all sides. On the other hand, writing alanine thus :-<smiles>CC(N)C(=O)O</smiles>

the central carbon atom is obviously differently weighted on all sides. By representing such an arrangement in space (P., p. 282), it can be seen that by interchanging positions of two of the groups a compound is obtained which is different in space arrangement, and cannot by any turning about be changed into the first one. It is, in fact, the image of it as seen in a mirror. All compounds which contain asymmetrical carbon atoms, that is, attached to four different groups, show the same characteristic, which is, indeed, a geometrical necessity. There are then two "isomers" of each of these compounds. How can we distinguish them? It is by 
their behaviour to polarised light. A beam of ordinary light consists of a number of ether elements vibrating in all possible directions at right angles to the direction of the beam. If looked at endwise, these vibrations fill up, as it were, the whole cross section of the beam, in all directions across it. There are, however, certain crystals which, owing to their structure, only allow vibrations of one particular direction to pass through, the others being blocked out or absorbed. The same thing can also be done by reflection from glass at a particular angle, in which case all the vibrations except those of one direction pass through; those of the particular direction are reflected. The beam is then said to be "polarised," because it has properties different in one direction from those in another direction. Suppose such a beam to be sent through a compound which contains asymmetric carbon atoms. Owing to their being different in one direction from that in another, such atoms will turn the plane in which the polarised light is vibrating through a certain angle. They are said to rotate the plane of polarised light, and to be "optically active." Now, according to the side of the carbon atom which is the more heavily weighted, the plane of the polarised light will be turned either to the right or to the left. Hence the two kinds of "optical isomers," as we may call them now, can be distinguished. That particular form of glucose, which is the only one utilised by the organism, rotates to the right; that form of alanine used rotates to the left. The means used for the detection and measurement of the degree of this rotation of polarised light is the instrument called the "polarimeter" (E., p. 181). The principle of it is this: light sent through the instrument is first polarised by a prism of Iceland spar cut in a particular way. At the eye end there is another similar prism which can be rotated, and the angle of rotation measured. If the plane of vibration of the light passed by the polarising prism is the same as that passed by the second prism ("analyser"), the light reaches the eye. If not, there is darkness. When a solution of an optically active substance is placed between the prisms, the analysing prism requires rotation in order to correspond with the plane of vibration of the light which has been rotated by passing through the solution. In the actual instrument there is a device which increases its sensibility, so that very small differences of rotation can be measured accurately.

It appears that living organisms must have first made their appearance under the influence of some asymmetrical forces, so that they developed a bias towards one set of optical isomers. Once established, this would tend to become more and more exaggerated. The question is a difficult one, but it must not be 
supposed that the production of optically active compounds is confined to the living organism, as sometimes suggested. All compounds with asymmetrical carbon atoms must be optically active. The point is that in the laboratory the two oppositely rotating isomers are nearly always formed in equal amount, so that the actual rotation is zero. The living organism produces one only, because the formation takes place by means of asymmetric agents, which are themselves already optically active, since they consist of one isomer only.

\section{The Green Plant}

Since animals cannot do with less complex sources of carbon and nitrogen than glucose and amino-acids, we have next to inquire where the supply comes from. They are only found in nature in the bodies of animals and plants. These bodies, or materials extracted from them, are taken as food by other animals. After being used they are rejected in simpler forms, deprived of energy, the carbon in great part as carbon dioxide (E., p. I82), the nitrogen combined with part of the carbon mostly as urea (E., p. I82), but sometimes in other more complex forms. None of these will serve again as food, until they have been built up by the supply of energy to more complex forms.

A word of explanation is needed as to urea. The whole of the nitrogen contained in the protein food is not needed for repair purposes, and urea is the way in which the waste ammonia groups are got rid of by combination with carbon dioxide. Urea is obtained from ammonium carbonate by removal of water, and can easily be reconverted by hydrolysis. Thus :-<smiles>NOOC(=O)ON</smiles>

Thus urea is the diamide of carbonic acid.

There is, then, a continuous using up of available carbon by animals, and the same is true for plants, with the exception of certain special structures in the green plants. It is only by the aid of these that the life of both animals and plants on the earth is preserved from final extinction.

In the oxidation of food, not only are useful carbon compounds used up, but the oxygen of the atmosphere also. We have now to learn something about the wonderful mechanism by which they are both restored in the course of the same reaction. This is probably the most interesting mechanism that exists, as well as being that on which the continued existence of life on the earth depends. 
We have, first of all, to convince ourselves that a green plant is able to make use, in some way, of the carbon dioxide in the air as a source of carbon to build up the complex compounds of its own structure. These compounds afterwards serve as carbon food for animals.

If we grow a plant from the seed in such a way that it can get no carbon except from the atmosphere, we find, nevertheless, that its bulk increases far more than would be possible by the use only of the material originally present in the seed (E., p. 183). It must have obtained its carbon from the atmosphere.

Another experiment which should be made is the following (E., p. I83): Fill a vessel, under which a green plant is growing, with expired air from the lungs. Take a sample of the air at once and determine the percentage of oxygen and of carbon dioxide in it by gas analysis The oxygen will be low, the carbon dioxide high, as compared with atmospheric air. Expose to sunlight for a day or two. Determine the composition of the gas again. The oxygen will have increased, the carbon dioxide decreased. Therefore, oxygen has been produced from carbon dioxide. If the experiment be done in the dark, this will not happen. In fact, if the gas analysis is very accurate, the opposite will be seen to have occurred, namely, an increase of carbon dioxide and a decrease of oxygen, just as in animals. Moreover, if a colourless plant, such as a mushroom or other fungus, be used instead of the green plant, there will be increase of carbon dioxide and decrease of oxygen even in the light.

Two things, therefore, are required-light and the pigment that gives the green colour to plants. It is clear that the energy used in the process has come from light, and that the means by which it is utilised is the green pigment, called "chlorophyll," or "leaf-green." This substance has several remarkable properties, but that which concerns us most is its relation to light-energy.

The enormous quantity of radiant energy that we receive from the sun is transmitted in the form of transverse vibrations in the ether of space. The rate of these vibrations is of a wide range. The wave-length depends on the rate, when the velocity of propagation is the same, being the distance one wave has travelled before the next one follows it. The lowest rates, or longest wavelengths, are only perceived by us as heat when they strike on the skin. Those of a certain medium wave-length are perceived by the eye as light, differing in colour according to their wave-length. The most rapid vibrations, or shortest wave-length, the ultra-violet, are not perceived directly at all, but are capable of causing chemical actions of various kinds to occur. Those rays, which we call "light" can also produce chemical changes when they fall upon 
an appropriate system. It will be perfectly obvious that none of these rays can produce any change in bodies on which they fall unless they are absorbed. To produce change requires energy, and if the energy of the light is as great after passing through a body as before it impinges upon it, no energy has been given up to that body, and no effect produced in it. All rays from the sun can be converted into heat when absorbed, and their energy measured in this way. But, as we have seen, if we want the most efficient conversion of their energy into other forms, such as chemical energy, it must take place without previously passing through the stage of heat. This is ensured by the aid of certain coloured substances which absorb the energy of light, and enable it to effect chemical changes directly. "These substances are sometimes called "optical sensitizers," because they make it possible for a chemical system to absorb light of a wave-length which it would otherwise be unable to do. A familiar instance is the dye with which "red-sensitive" photographic plates are stained.

If we take a coloured solution, say one of a green tint, and look through it at a white surface, we realise that the light which reaches the eye must be that which has not been absorbed. A green solution absorbs the light of both ends of the spectrum, leaving the green part in the middle. If we next examine a solution of chlorophyll with a spectroscope (E., p. I 86), we find that there is a particular region in the red in which the light is greatly absorbed, showing a dark band in dilutions such that very little absorption is shown elsewhere. A spectroscope is an instrument which sorts out the mixed wave-lengths of white light in series, according to their wave-length. This it does by means of a prism, or other device, which deflects the rays from their straight course in proportion to their wave-length on account of the fact that in passing through the prism the red rays are turned aside less than the violet rays. They are deflected less because the rate of propagation of light waves in a dense medium like glass is lower than in air, and that of rays of short wave-length is affected more than that of the longer ones. So that when a wave-front strikes obliquely, more effect is produced on the shorter waves.

The reason why a substance absorbs rays of a particular wavelength is because the rate of vibration of certain of its molecular constituents coincides with that of the light absorbed. The energy of the light is thus transferred to the absorbing substance by what is known as "resonance." This may be understood by taking a pendulum at rest and giving a series of very slight blows. The first of these will produce a very small movement of the pendulum, which will swing back beyond its resting position and then return 
in the direction in which it was driven at first. If the second blow arrives just at the time in which this last movement starts it will increase it, and a repetition of the blows at the correct moments will finally result in a vigorous vibration of the pendulum. We have converted the energy of the blows into a movement of a large mass. Unless the blows are timed to the natural rate of the pendulum, some of them will push it in the wrong direction and undo the work of the rest. The energy of blows so delivered, instead of setting the system into its natural rate of vibration, will be wasted as heat. Similarly, the light energy taken up by resonance is converted into molecular movement of the natural rate, and may so increase this movement that chemical change occurs, and is thus used in chemical work without becoming heat.

What, then, is the reason why chlorophyll has this especially great absorption in that part of the spectrum which we see as red? It has been found by measurement that the energy of the rays in the solar spectrum is greatest about the yellow. This is due to the fact that these measurements were made on a high sun in a clear atmosphere. Since the atmospere absorbs rays of short wavelength more than it does those of the longer wave-length, it is possible that the position of the chlorophyll band may be in that of maximum energy for the greater part of the day and the greater part of the weather, especially in the higher latitudes.

So far we have seen what provision is made for absorbing radiant energy, and we next inquire as to the chemical changes which it causes to take place. We saw that the final result is that carbon dioxide is decomposed and that oxygen is given off. Let us take the carbon dioxide part of the problem first. It is easy to show that starch is the final product (E., p. I86). Now starch is an insoluble carbohydrate formed by the union of a large number of glucose molecules by removal of the elements of water from two neighbouring ones, in a similar way to the union of two aminoacids described above. The advantage of its being insoluble will be seen later, when we come to learn about enzymes. The formation of starch from glucose is not a result of the light, so that what we have to account for is the production of glucose or a similar hexose. From the composition of glucose we see that hydrogen has to be introduced into the molecule of carbon dioxide to start with. This, of course, comes from the water present. The final net result is :-

$$
6 \mathrm{CO}_{2}+6 \mathrm{H}_{2} \mathrm{O}=\mathrm{C}_{6} \mathrm{H}_{12} \mathrm{O}_{6}+6 \mathrm{O}_{2}
$$

and we see that there is a large increase in chemical potential energy, which has come from the sun. But this process must consist of several stages. What is known about these? We 
have seen that the simplest compound of the same percentage composition as glucose is formaldehyde, $\mathrm{CH}_{2} \mathrm{O}$, and it is natural to imagine that this might be the first result of what is sometimes called "photo-assimilation," the taking up of carbon by the agency of light. Although there is much probability that this is the case, from the general properties of formaldehyde and other evidence, it has not yet been found possible to obtain really satisfactory proof of its production in the green leaf. This may be due to the fact that it is a powerful chemical agent, and injurious to living protoplasm if present in any but minute quantities. For this reason it would be rapidly converted into sugar. In the laboratory it can easily be caused to unite, six molecules at a time, to form a hexose. A further ground for the belief that formaldehyde is formed by the action of light in the chlorophyll system is that certain artificial systems, under the action of light, can produce it from carbon dioxide and water. One of these is colloidal ferric hydroxide under the action of ultra-violet light. This fact is of further interest, because it suggests a possibility with regard to the mechanism in the plant. To understand this we must consider the form in which chlorophyll is present in the cell. It is insoluble in water, and is therefore present in particles or colloidal solution, and is not distributed generally throughout the cell substance, but located in special structures usually, but not always, of spherical shape (E., p. I 86). These "chloroplasts" contain other things in addition to chlorophyll, and of some of these it is interesting to find that iron is a component. Although iron is very common in cells, and has functions connected with oxidation, its presence in the chloroplast is significant, and suggests that the function of the chlorophyll itself may be to absorb light energy, bringing it into intimate relationship with the chemical system, and that, after absorption, the iron may come into play and cause the production of formaldehyde, as in the ferric hydroxide above.

At the same time, chlorophyll itself has so peculiar a chemical structure that it is difficult to believe that this does not, in some way, play a part. The suggestion has been made that carbon dioxide and water are taken into combination with the pigment, then reduced to formaldehyde, which is given off. But no evidence exists that chlorophyll takes up carbon dioxide. By itself, even in the presence of light and of carbon dioxide, it does not produce formaldehyde or sugar. It appears that the other parts of the chloroplast are necessary.

Although the chemical nature of chlorophyll has not, as yet, thrown much light on the photo chemical reaction with which we are concerned, this structure is in itself an interesting one. In connection with the possible function of iron, it is remarkable that, 
although this element is not a part of the molecule of chlorophyll, yet if a plant be grown from the seed in absence of iron, no green pigment is developed until iron is supplied. Chlorophyll, or rather the important green part of the molecule is, briefly, a number of pyrrol derivatives, four to be exact, united by magnesium. Thus the magnesium is in organic combination. On the other hand, the red colouring matter of the blood, hæmoglobin, is a similar pyrrol derivative in which iron takes the place of magnesium. The properties of hæmoglobin are as remarkable in another way as those of chlorophyll are, as we shall see presently. The structure of pyrrol is that of a ring of four carbon atoms and one nitrogen atom, each united to hydrogen, and is produced from proteins by destructive distillation. In those derivatives which form chlorophyll and hæmoglobin, two of the hydrogens are replaced by methyl and one by ethyl. The magnesium may be removed by the action of acids, without destruction of the green colour, a fact which makes its presence somewhat puzzling, if the function of chlorophyll is merely that of absorbing light of a particular wave-length.

On the whole, it must be admitted that we know little about the mechanism. The system is a very complex one, and photochemical reactions, even of a simple kind, are still obscure in many respects.

The way in which oxygen is produced is still more difficult to explain. We can only point out how it might happen, on the basis of certain facts which are known. It is not an uncommon action of radiant energy to bring about the formation of peroxides, raising the chemical potential of oxygen. Peroxides are oxides containing more oxygen than the simple oxides. Thus, water being $\mathrm{H}_{2} \mathrm{O}$, the peroxide of hydrogen is $\mathrm{H}_{2} \mathrm{O}_{2}$. This may be represented either as $\mathrm{H}-\mathrm{O}-\mathrm{O}-\mathrm{H}$, or if the rise in potential of oxygen implies $\mathrm{H}-\mathrm{O}$

its becoming quadrivalent, as

In either case, the extra $\mathrm{H} \rightarrow \mathrm{O}$.

oxygen atom is readily available for oxidising other substances, or being set free from two molecules together, given off in the form of gaseous molecular oxygen. If, in any way, peroxides were produced in the leaf under the action of light, oxygen could be obtained from them under appropriate conditions. There is evidence that peroxides are formed in chlorophyll systems by light, although it is not certain that they do not arise from destructive oxidation of the pigment itself. Such organic peroxides give rise to the production of hydrogen peroxide by interaction with water, Further, there is an cnzyme, called catalase, present in all green leaves, which decomposes hydrogen peroxide with the evolution of gaseous oxygen. This is about as far as we can go at present. 
We see, however, how dependent we are on the sun for our continued existence, and more definitely how the "struggle for existence" is one for the possession of the free energy of the sun's rays.

The assimilation of carbon dioxide and the production of oxygen is the great function of the enormous area of green leaves that is to be seen on the earth. Since it is necessary for them to receive as much light as possible, we see why they are in the form of thin sheets, and why they spread themselves out in such a way as to receive the maximum amount of light. Sometimes in the tropics the sun's light is destructively brilliant, and the chloroplasts take such positions as not to be subject to its full intensity.

\section{The Cycle of Nitrogen}

As already stated, waste nitrogen leaves the animal body chiefly in the form of urea, a small amount as more complex compounds. When death takes place, the proteins of the tissues are broken up by the agency of those minute vegetable organisms called "bacteria." In the case of plants, although their protoplasm probably gives off some simple substance, such as urea, in the course of its chemical changes this is very small, and the nitrogen of the plant structures finally passes to the soil in the same way as that of the animal body does.

What, then, are bacteria?

They are microscopic plant organisms, devoid of chlorophyll, and exist in great variety of forms and properties (E., p. I86). They are present all around us, to some extent blown about in the air, but chiefly on the surfaces of all kinds of materials and in the waters and soil. One of the most striking things about them is that, although the number of distinct shapes assumed is not great, the chemical activities they perform are of an enormous variety. The chief forms are: small spheres (micrococci), sometimes attached together in chains, bacteria (short rods with rounded ends), bacilli (longer rods with flat ends), and twisted rods of various lengths and closeness of coils (spirilla and vibrios). Some of these in certain stages move about by the agency of threads which are contractile (flagella). Others are frequently found in a resting state as spores, surrounded by a layer of material which makes them very resistant to the action of heat. The name "bacteria" is commonly used as a general name for the whole group. The name "micro-organisms" includes also yeasts, moulds, and small animal organisms, protozoa, of which amœeba is one. Protozoa are abundant in the soil and in stagnant water.

Those bacteria concerned in the destruction of animal and plant remains are the cause of what is known as "putrefaction," the final 
result of which, in the case of nitrogen compounds, is ammonia or closely related compounds. Many of these micro-organisms are responsible for certain diseases, owing to the production by them of poisonous substances, "toxins," which have powerful actions on physiological processes, differing according to the particular organism producing them. In order to avoid putrefaction, those bacteria already present must be killed and access of others prevented. This is the process known as "sterilisation," familiar in the domestic operation of bottling fruit. To avoid the access to wounds of those organisms causing disease was the object of the "antiseptic method" introduced by Lister. In his time the hospitals were swarming with noxious organisms to such an extent that it was necessary to dress wounds with chemical substances destructive of bacteria. With the steady progress of general destruction, the use of antiseptic chemicals has become less necessary, and the sterilisation of the hands and instruments usually sufficient. But it must not be forgotten that the principle on which Lister worked was the exclusion of infection by any method whatever. The modern "aseptic" method is merely one form of his treatment, made possible by the previous antiseptic methods. It is clearly a difficult matter to find an antiseptic chemical which kills bacteria without injury to the delicate new tissues growing in a wound, although some progress has been made in this direction. The need of it has been made evident by the wounds of the late war, which naturally became infected with all kinds of organisms.

There are then in the soil micro-organisms which convert the remains of animals and plants into ammonia compounds. Urea and other nitrogenous excreta are also converted into ammonia by the same agency. Now, green plants and some fungi can make use of ammonia as a source of nitrogen, but it is rather remarkable that green plants do better with nitrates. Indeed they are said to suffer from nitrogen starvation when ammonia is their only supply of nitrogen. It would have been expected that this would more readily yield the $\mathrm{NH}_{2}$ groups required for the production of aminoacids and proteins.

A part of the ammonia is probably made use of by the plant, but the greater part is oxidised in the soil to nitrates by certain bacteria present therein. The first stage is the production of nitrites by a particular group of organisms. Another group then converts the nitrites into nitrates (E., p. I86). The green plant is thus supplied with that form of nitrogen food which it can utilise best. Animals then consume the plants as sources of protein, and so the circle is completed. But not entirely, since during the conversion of the residues to ammonia some of the nitrogen is lost, apparently by the agency of oxidising bacteria, becoming 
atmospheric nitrogen. Moreover, there are in the soil what are called "denitrifying" bacteria, which cause a loss in the nitrate unless it is rapidly used up by the plant. This loss involves return of nitrogen gas to the atmosphere.

Unless, therefore, there were some means of making use of nitrogen from the atmosphere, there would be a continual loss of nitrogen in the form in which alone it can serve as food for plants and animals. The student is probably aware that there are artificial processes by which the oxygen and nitrogen of the atmosphere are made to combine to nitrous and nitric acids, and others which combine nitrogen and hydrogen to form ammonia, which is oxidised to nitric acid by a further process (P., p. 253). But there is a natural process. There are bacteria in the soil which are able to utilise nitrogen from the atmosphere to form the material of their own bodies. When they die, this material serves as a source of ammonia to the soil. The actual chemical reactions by which nitrogen is made use of by these bacteria are not known, but it is clear that a supply of energy is required. This is provided for by oxidation of carbon compounds in the soil. Bacteria, with similar powers, are present in the nodules on the roots of the plants belonging to the order of the beans, clovers, etc., the Leguminosæ, and in rare instances in other orders (E., p. I87).

This last case is one of those known as "symbiosis," where organisms join together for mutual assistance. The leguminous plant supplies the bacteria with a carbon compound to oxidise, and receives in return material which serves it as a source of nitrogen. Readers of the "Georgics" will remember that Vergil advises farmers togrow vetches on their fields before sowing wheat. Another interesting case of symbiosis is that of a marine worm, in whose tissues cells of an alga containing chlorophyll are present. The animal's waste nitrogen serves for the plant cell, and this in turn, by aid of its chlorophyll, supplies carbohydrate to the animal (P., p. 295). We may learn a lesson from this. Much advantage is to be gained by mutual co-operation in making use of what is put at our disposal in the outer world. Waste of energy is involved in contest for its possession. Claude Bernard, the great French physiologist, has pointed out how much more inspiring it is to regard living beings as adapting themselves to surrounding conditions, rather than as being in perpetual conflict with them. The life of an animal, as he says, is part of the total life of the universe.

\section{Sulphur and Phosphorus}

The supply of these elements in organic combination is involved in that of nitrogen, since some of the proteins contain, as parts 
of their molecules, compounds containing them, so that they will be taken as food by the animal along with the other parts. The higher plants are able to make these compounds for the animal from inorganic salts, sulphates and phosphates in the soil. The animal also, to some extent, uses inorganic compounds of sulphur and phosphorus.

We may note again here that there are certain constituents in protein food which cannot be made by the animal organism itself, and must be supplied. They are probably required for the replacement of particular parts of the cell machinery, although it is also possible that important chemical products need certain chemical groupings to be provided, the animal cell being unable to make them.

\section{Salts}

Inorganic salts are found to be present in living cells, and in the food taken by living organisms. But the question may be asked, Are they necessary, or only present because food materials always contain them?

The relationship of colloids and salts, briefly discussed in the preceding chapter, indicates that salts must play an important part in the colloidal changes of the cell. It has been found by experiment that certain inorganic elements are necessary, not only for growth, but for the proper working of the activities of the living cell.

The heart of a frog can be made to continue beating if supplied with a solution of inorganic salts only. We have learned in our study of the osmotic pressure of cell contents that such a solution must possess a particular osmotic pressure, otherwise the tissue cells either swell or shrink. We can give this value to our solution by sugar or by sodium chloride. It is usual to do so with the latter, because of its convenience; but there is good evidence that sodium chloride is somewhat toxic, and that a part of it may, with advantage, be replaced by its osmotic equivalent in cane sugar. Even if we do this, however, we find that we cannot keep the heart beating normally for more than a short time (E., p. 187). We find that both calcium and potassium are necessary in small amounts, and that there is a certain proportion between the three cations that gives the best results. It may have been noticed that no mention was made of any particular salt of these metals; in fact, the anions may be of various kinds indifferently. This must not be taken to imply that the anion plays no part, but rather that its function is one common, more or less, to all anions, apparently due to the sign of the electrical charge. Certain special properties, on the other hand, are required in the cations. Calcium ions, 
as we saw, are active in the way of aggregating or precipitating colloids, but there are probably other properties to be taken into account. Potassium appears to be of importance on account of its radio-activity.

There is an interesting and suggestive fact about the salts necessary in a solution to take the place of blood. Suppose that we take sea water and dilute it so that its osmotic pressure is the same as that of blood. We find that it serves excellently as an artificial fluid, so far as the salts are concerned. Examining it more closely, we notice that the proportion of sodium, potassium, and calcium salts is practically the same as that found to be the best in a mixture made for the purpose. Is this merely accidental? The blood, as we find, contains the salts of the ocean such as they would be if sea water were less concentrated than it is now. But we know that it has been, through geological ages, continually increasing in salt content, because rivers are always adding salts dissolved from the land by rain, whereas it is only water that evaporates from the ocean. At some period, then, its composition was similar to that of the present land vertebrates as regards inorganic salts. When the ancestral vertebrates, which were formed in the ocean, left it for the land, there is every reason to suppose that the salt content of their blood would be the same as that of the ocean, and that their cell mechanisms would have been adjusted in relation to it. Hence it remained at this point. The geologists tell us that this taking to life on land occurred about the end of the Cambrian period. This period was one of great length, judging by the thickness of the rocks; so that ample time had passed for the adjustment of the cell mechanisms to the composition of the ncean. We may take it that the blood represents the salt content of the ocean at the end of the Cambrian period. There is, however, one point which requires some further explanation, namely, the high content of the sea at the present time in magnesium salts, which is out of proportion to the other constituents, as compared with blood. There are reasons for believing that magnesium has increased more than the other salts, but further discussion would lead us too far (P., p. 210).

A further conclusion is suggested. Perhaps the salt content of the cells, which is not identical with that of the blood, may represent the composition of the ocean at a still earlier period. But there are difficult questions involved here.

The variety of salts required for growth, at all events in the case of plants, and as far as we know in that of animals also, is greater than this. The ordinary mould, Aspergillus, requires for its most rapid growth magnesium, potassium, zinc, and iron as cations; phosphate, sulphate, and silicate as anions. A sea weed 
was found to require at least sodium, potassium, calcium, and magnesium.

\section{Accessory Factors}

We have seen that we must provide for a supply of carbon and nitrogen in certain forms, and also salts. But there is something else to be considered.

Suppose that we give to a growing animal a diet of pure protein, pure fat and pure carbohydrate, together with salts, that is, all the actual chemical compounds required and in sufficient amount, we find that it does not grow. But if we add a very small quantity of milk or of turnip juice it grows as well as on its normal food. There is evidently something wanting in the pure materials, of which only a small amount is needed, but which is, nevertheless, indispensable.

We do not know yet what this "accessory factor" is. It has been called "vitamine," owing to a mistaken view of its chemical nature. There is ground for believing that there are several kinds, because different diseases develop in the absence of particular constituents present in some foods, not in others. For example, beri-beri, after having made its appearance, can be cured by the addition of a small quantity of the outer layer of rice, whereas scurvy cannot be cured by this, but needs the juice of oranges or other fresh vegetable. This latter fact was known to Captain Cook, who discovered, in his second voyage round the world, that scurvy, then so serious a difficulty in long voyages, could be prevented by adding fresh vegetables to the preserved diet whenever a chance presented itself. Some other diseases are turning out to be, in all probability, "deficiency diseases."

Although there seems to be some variety in these factors, they fall into two main groups, one soluble in fat ("fat-soluble A-factor"), the other soluble in water ("water-soluble B-factor"). That contained in butter is typical of the former, that in wheat-germ, of the latter. Both are necessary for normal growth.

These factors are somewhat easily destroyed by cooking, especially if heated in alkaline solution, and by preservation with salt. Hence the importance of fresh food, especially fruit and vegetables. Fruit contains an unusually large amount of the anti-scorbutic factor, which seems to belong to a special class.

Notwithstanding their great importance, very little is known about the nature of these substances or the way in which they act. They seem to behave like those agents called "catalysts," about which we shall learn more presently. They are not subject to chemical change in the course of their activity (P., pp. 258, etc.). 


\section{The Supply of Energy}

Up to the present we have chiefly considered the things necessary to make new structures or to replace those worn away. Only incidentally have questions concerning energy been touched upon.

It was stated above that, in the adult, there is very little loss in the wear and tear of the cell machinery in its normal work. There is some loss by destruction of cells on the surface of the body and elsewhere.

The fact that the machinery does not wear away in its normal function has been shown most clearly in the case of muscular work. Since the cell structures contain nitrogen, if there were disappearance of their material there would be found an increased amount of nitrogenous compounds in the urine, since this is the way in which they are got rid of. The most careful investigation has shown that no increase is to be detected.

The necessity of taking more food than that indicated by such considerations as those above is a matter of general experience, so that there is another purpose which actually requires the provision of something other than the material itself. As already pointed out, this is energy, which has been lost in the performance of work, and must be replaced.

The food we take is, chemically, of such a kind that by oxidation, or burning with oxygen, energy is given off. If we burn sugar or fat in the air (E., p. 189), we notice that heat is produced, and if we collect the gas given off, we find that carbon dioxide and water have been formed. We saw previously that these are also produced by the living organism when it makes use of the same substances. The chemical energy which appears as heat when the substance is burned in air is therefore available for the needs of the organism when similarly burned in its cells. But the mechanism of the living cell is so arranged that it can seize upon this energy before it has become degraded to heat, and so make more economical use of it.

In the preceding chapter it was shown that any form of energy can be converted into heat, and therefore measured in terms of heat units. This is a matter of some convenience in respect of materials used for food. We can burn them with oxygen and measure the heat produced, thus obtaining their value as sources of energy, on the assumption, of course, that they are such as the body is capable of using in this way. Charcoal or coal gas are useless, because the living cell does not possess the means of burning them. In the case of carbohydrates and fats, the values are those actually obtained by the organism, since these are completely oxidised by 
it. But the nitrogenous part of the protein used for energy purposes is not completely oxidised. Urea, when burned with oxygen, gives a certain, not very large, amount of energy, but it must be allowed for in calculating the energy value of proteins.

The unit of heat in which the energy value of food is expressed is the large calorie, in physiological discussion usually spoken of as the calorie simply. This is the amount of heat required to raise the temperature of one kilogram of water by one degree centigrade, or more precisely, to raise its temperature from $0^{\circ}$ to $I^{\circ}$, since the specific heat of water varies somewhat at different temperatures.

The performance of a given amount of work by our muscles requires the provision of its equivalent quantity of energy in food. This is obvious as regards work done on objects in the outer world. But a living organism differs from a machine which consumes no energy when at rest, in that the heart must go on, breathing must go on, and some other functions require energy even when the body appears to be at rest. It may rather be compared to such a machine as a circular saw, which is kept running when not actually occupied in sawing wood. A certain quantity of energy is used in friction and in fanning the air, but the consumption is greatly increased when a beam of wood is sawn.

The consumption of energy for the internal needs of the organism is known as the "basal metabolism," or basal consumption. This is the first time that we have used the word "metabolism," and it needs definition. It is simply a convenient word to express, briefly, the series of chemical changes undergone by chemical compounds in the organism. Thus the metabolism of carbohydrate means the various stages through which it passes before final oxidation to carbon dioxide and water.

The actual amount of energy required for basal needs varies, naturally, with the size of the organism. It is almost exactly one calorie per hour for each kilogram weight in man when asleep; so that, for a man of average weight (70 kilos or I I stone), it amounts to I700 calories per day. The amount required when external work is done differs according to the amount of this work, but the following table shows the approximate accepted values:-

\begin{tabular}{|c|c|c|c|c|}
\hline Basal, in sleep - & - & - & - 1,700 & lories. \\
\hline Do. awake, but at res & & - & $-2,100$ & 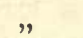 \\
\hline Sedentary occupation & - & - & $-2,500$ & , \\
\hline Light work & - & - & - 3,000 & \\
\hline Moderate work - & - & - & $-3,500$ & " \\
\hline Heavy work - & - & - & $-4,000$ & 9,00 \\
\hline
\end{tabular}

Carbohydrate alone might give the energy required; but we have seen that protein is necessary to afford nitrogen for replacement of wear and tear, and since it also gives energy, a diet might 
be composed of it alone. But it would be wasteful, because the greater part of the nitrogenous component is excreted unused. There is some popular misconception implied in the name sometimes given to proteins as being "flesh-formers," as distinguished from carbohydrates, which are said to be "heat-givers." It is unnecessary to say that protein must be supplied when new tissue is being formed; but this is a very different thing from the suggestion that it will form flesh (i.e., muscle) of itself alone. If the muscles are exercised, they may increase in bulk, and to do this a small amount of nitrogen is required. On the other hand, a food giving heat is equivalent to saying that it gives energy in general, and the name applies to protein as well as to non-nitrogenous food. It was, indeed, supposed at one time that there was some special value in protein as a source of energy, but exact observations have been unable to confirm this view. The names "flesh-formers" and "heat-givers" are quite unscientific, and do not correspond to any real distinctions. They should be given up altogether.

Not very much has been said, as yet, with regard to the third class of substances used for food. These are the fats. They consist of carbon, hydrogen, and oxygen, like the carbohydrates, but the hydrogen is present in larger proportion than required to combine with the oxygen to form water. Accordingly they afford, when equal weights are oxidised, more energy than carbohydrates do. Otherwise there does not seem, so far as can be made out, any physiological necessity for fat as there is for protein and carbohydrate. There is, undoubtedly, a desire for it, but this may be for reasons of making dishes attractive to the palate. It has been found possible for strong, healthy men to live without fat for two years. The presence of a particular accessory factor, the "fatsoluble A," in some fats makes their use advisable, although the factor is not confined to what are generally called fats.

As to the chemical nature of fats, they are what are known as "esters," a large class of compounds in which an alcohol residue is united with an acid residue. Alcohols are characterised by the presence of a $\mathrm{CH}_{2} \mathrm{OH}$ group, united with carbon and hydrogen. If the alcohol group is combined with hydrogen alone, we have methyl alcohol, $\mathrm{CH}_{3} \mathrm{OH}$; adding $\mathrm{CH}_{2}$, we get ethyl alcohol, $\mathrm{C}_{2} \mathrm{H}_{5} \mathrm{OH}$, and so on up to a large number, when the compounds become solid. The additions are not necessarily made in a way to form a straight chain, hence we have different alcohols with the same number of carbon atoms. The group $\mathrm{CH}_{2} \mathrm{OH}$ may be attached to other more complex groups than in the fatty acid series above referred to, but it is this series that interests us more especially here. There may also be more than one alcohol group, as in glycerin (more correctly, glycerol, since the termination ol has been agreed upon as that of 
an alcohol). Glycerol has three alcohol groups united together by loss of hydrogen from the middle one :-

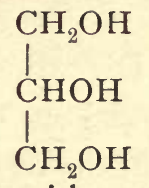

The acid in an ester may-be either organic or inorganic, and the combination takes place with the elimination of the elements of water. Ethyl alcohol and acetic acid unite thus :-

$$
\mathrm{C}_{2} \mathrm{H}_{5}(\mathrm{OH} \quad \mathrm{H}) \mathrm{COOCH}_{3}
$$

forming what is often called ethyl acetate; but since it is not a salt, its correct name is acetic ethyl-ester. The acidic properties of the acid have disappeared, although not by combination with a base.

Ordinary fats and oils are esters of glycerol with fatty acids of a large number of carbon atoms. Thus, olive oil or olein is the tri-glyceride of oleic acid, having three oleic acid residues united to the alcohol. Oleic acid contains eighteen carbon atoms, while the stearin of mutton fat is the corresponding ester of an acid also containing eighteen carbon atoms. The well-known difference in their properties is due to the smaller number of hydrogen atoms in oleic acid, some of the carbon atoms being unsaturated. Reference will be made to this again later.

There is an interesting group of complex fatty substances called "lipines," which form important constituents of cells, although their function is not altogether clear. One of their uses is doubtless to take part in the production of the cell membrane, but there must also be some more distinctively chemical part to be played by them. Probably they take some share in the oxidative mechanisms. Some of these contain phosphorus and nitrogen, such as lecithin, which may be regarded as phosphoric acid combined with a fat and an organic nitrogen base. Others are devoid of phosphorus, but contain fatty acid, a nitrogen base and a sugar, and are found chiefly in the brain.

The relative proportion of the three kinds of constituents of a diet may be varied to a large extent without injury to the healthy individual, so long as the energy value is not decreased. The Royal Society Food Committee recommends the following as the basis of calculation for the food supply of a nation:-

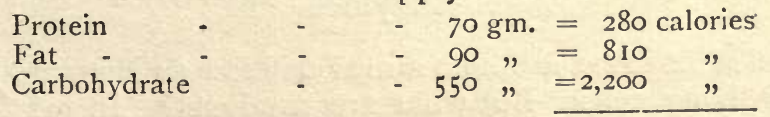

per day per 7o-kilo man, doing moderate work. 
Tables have been constructed, giving the composition of various articles of food and their calorie values.

\section{Digestion}

The fats, carbohydrates, and proteins contained in the various articles of our diet are not in the form in which they can be made use of by the living cells, whether for growth or energy purposes. Carbohydrate must be in the form of glucose or fructose ; protein in that of amino-acids. That in which fat is required to be is unknown, but it cannot be absorbed in the form found in articles of food. The necessary changes in all these cases are made in the alimentary canal.

The primitive form of the alimentary canal is that of a tube passing through the body, open to the exterior at both ends. At the anterior end, the mouth, the food is taken in. In its passage it is subjected in turn to the action of various fluids and the products absorbed. Finally, the constituents which resist the action of the digestive juices are expelled through the anus. The processes to which the food is subjected are essentially the same in all animals, so that we may take the arrangements present in one of the higher vertebrates for description of the whole series of events, which can be analysed in such a case much more accurately than in small animals.

We need spend but little time on the mechanical disintegration necessary in the case of certain materials, on account of their being united together in more or less dense masses. This is done by the teeth in mammals; in birds which eat hard grains there is a muscular organ, the gizzard, which contains small stones, serving to grind up the food. In the mouth cavity the food is moistened by a liquid, the saliva, which is poured in along tubes leading from special organs which secrete it, as the process of its formation is called. In some animals, including man, saliva contains an agent which brings about the conversion of starch to sugar. This is the first of a series of agents acting in a similar way as the food passes along the alimentary canal. They are known as "enzymes," and before we proceed further we must learn something about the manner in which they act.

\section{Enzymes}

These substances are sometimes defined as the catalysts produced by living cells. But what are catalysts? In brief, we may say that their action is to make chemical reactions proceed at a faster rate than they naturally do, and that they do this without 
themselves suffering any permanent change. They reappear unaltered when their work is finished.

That chemical reactions vary greatly in the rate at which they proceed is familiar (E., p. 189). We have already noted that comparative slowness of reaction is characteristic of carbon compounds. In the processes of the living organism, it is of importance that reactions should not go on at a perceptible rate except when required. Hence the value of catalysts to quicken them up at appropriate times. In the cell these catalysts, or enzymes, are produced or brought into an active state as wanted.

When we say that what we have to do with is an acceleration of chemical changes which proceed of themselves, although but slowly, we must remember that this rate may be so slow as to seem not to take place naturally at all. Since, as we stated above, the enzyme appears at the end unaltered, it is obvious that it does not give up energy to the reacting substances, and therefore that the final result must be the same as it would have been without the presence of the enzyme. There is a certain qualification, however, which must not be passed over, although it does not alter the general principle. Owing to the fact that enzymes, as we shall see, form a separate phase of the system in which they act, and the conditions at boundary surfaces differ from those in homogeneous systems, the final state of equilibrium arrived at is not necessarily the same in both cases. The explanation of the fact is still obscure, and need not detain us at the present stage.

An important consequence follows from such considerations, and is found experimentally to be the case. An enzyme acts in opposite ways, according to the state in which the reagents are when subjected to its influence. This will be clear if we take a special case. Fats, as we saw, are esters in their chemical nature, and there are enzymes which bring about a splitting of esters in general into their component acids and alcohols. This they do by introducing water. Thus, taking ethyl acetate and putting Et for ethyl $\left(\mathrm{C}_{2} \mathrm{H}_{5}\right)$ and $\overline{\mathrm{A}}$ for acetyl $\left(\mathrm{CH}_{3} \mathrm{COO}\right)$ we have :-

$$
\overline{\mathrm{Et}} \overline{\mathrm{A}}+\mathrm{H}_{2} \mathrm{O}=\overline{\mathrm{Et}} \mathrm{OH}+\mathrm{H} \overline{\mathrm{A}}
$$

Although this reaction only takes place rapidly under the action of a catalyst, it proceeds at a detectable rate if allowed to proceed by itself, but never completely. If we start with ethyl acetate and water in the proportions in which they combine, that is, if we take an equal multiple of the molecular weight of each, we find that after some days we can detect the presence of acetic acid (E., p. 190). After a long time it will be found that no further change is going on, and that we have a state which we call equilibrium. By estimating the amounts of the four com- 
ponents present, it is found that there are twice the number of molecules of the ester and water that there are of alcohol and acid. Now, suppose that we begin with alcohol and acid, we find that they combine to form ester until a certain amount has been produced. The reaction then stops, and on examination we find that the proportion of the four components is the same as in the former case. When the reaction is accelerated by a catalyst, similar conditions hold, since no energy is supplied by it. Therefore the same catalyst may appear to have either synthesising or hydrolysing powers, according to the substances on which it acts (E., p. I90).

The position at which no further change takes place is called an equilibrium, or the reaction a balanced one, because what is happening is that the two opposite reactions are both proceeding at equal rates at this point.

Our problem is, then, one of velocity of reactions. The fundamental law concerned is that of mass action, which states that the rate at which a particular reaction takes place is proportional to the mass of the reagents present in a certain volume. It follows that the relative amounts of the original reagents and of their products present in the final equilibrium is also proportional to their original masses. Thus, if we increase the mass of water in the example given above, we correspondingly increase the rate of the hydrolytic reaction, and since the rate is maintained greater, relatively to the opposite one, also in the equilibrium position itself, there will be present finally a larger proportion of acid and alcohol than if there were less water present to start with. When the proportion of water is great, the synthetic reaction may be so slow that it is difficult to detect it, and the final result may appear to be one of complete hydrolysis.

In stating the law of mass action correctly, it is necessary to say that the rate of the reaction is proportional to the concentration of the active masses of the reagents. The reason for inserting the word "active" will be clear if we suppose that we are dealing with a reaction brought about by hydrogen ions, and that the acid used is a weak one. The active mass is not that of the acid added, but that part of it which is electrolytically dissociated.

The word "concentration," or mass in unit volume, is necessary, as can be seen by realising the kinetics of the process. The reaction takes place because, as the molecules shoot hither and thither, some of them hit those with which they enter into combination. It is only a certain number of these encounters that actually result in combination, but it is obvious that the number of effective meetings that take place in unit time, that is the rate 
of the reaction, is the greater the more molecules there are in the way to be hit against.

We saw above that the essential property of a catalyst is that of increasing the rate of a reaction. Their importance is therefore greatest when the reaction is, by nature, a very slow one, and this applies in a marked degree to those of living systems.

There are two ways in which catalysts act, according to whether they are in true solution, forming homogeneous systems, or present in a distinct phase, as a solid or in colloidal solution, forming a heterogeneous system. In the former case the effect is known to be due sometimes to the formation, first of all, of a compuund between the catalyst and the components of the reacting system. This compound is then decomposed rapidly with separation of the products of the reaction and the reappearance of the catalyst itself. The whole process takes place more quickly than the reaction proceeds by itself. It must be admitted, however, that there are cases of homogeneous catalysis to which this explanation is not readily applied, and although the production of intermediate compounds is not altogether excluded as a stage in the action of enzymes, it does not concern us here, because our catalysts, the enzymes, are in colloidal solution and act, in some way, at the surfaces of contact with the solution containing the reacting substances. Can we form any conception of what happens?

By the law of mass action, if we can increase the concentration of the reagents, we shall increase the rate of the reaction. Further, we have seen that if substances decrease surface energy, they are concentrated by adsorption on the surface. What evidence is there in the case of enzymes or other heterogeneous or insoluble catalysts that adsorption plays a part? Let us examine a fairly simple case, that investigated by Faraday, where platinum surfaces were found to bring about rapid combination between oxygen and hydrogen gases. Strong evidence was brought in support of the view that this was due to condensation of the gases on the surface of the platinum, and in this way brought within range of the forces causing their combination with each other (P., pp. 306 and 326). It was shown by Faraday that the surface must be perfectly clean; that is, if another substance had already obtained possession, oxygen and hydrogen were kept out to a great extent. Moreover, chemical reaction with the platinum, forming intermediate compounds, was excluded. No matter how the surface was cleaned, by mechanical or by oxidising or reducing agents, the method was effective. Chemical reaction between platinum and oxygen is also excluded by the facts that nitrous oxide and hydrogen are caused to combine, and that similar effects "are produced by most, if not all, solid bodies." 
Is there any evidence of similar action in the case of enzymes? What evidence there is, is naturally more or less indirect; but it is in favour of a preliminary adsorption of the reacting substances on the surface of the enzyme. It lies, in great part, in the facts concerning the rate of the reaction in relation to the amount of the enzyme present, and cannot be discussed here (P., p. 315). There is also an interesting retarding effect of certain inert substances, such as saponin, whose action, as a whole, can only be explained by its great lowering of surface energy, thus obtaining possession of the enzyme surface, and displacing more or less the other constituents of the system from that surface. This is similar to the effect of impurities on Faraday's platinum, and -it has the negative temperature coefficient characteristic of surface tension.

But there are a great number of enzymes known to us, each of which causes acceleration in one particular kind of reaction only, a fact at first sight difficult to reconcile with mere condensation on a surface. It might be supposed that one enzyme only would suffice for all purposes. At present, however, we have not sufficient knowledge of the numerous properties of surfaces to be able to exclude the probability that surfaces of different chemical structure have different powers of adsorption with regard to the various chemical natures of the components of the reactions which they influence. It is, moreover, not improbable that the rate of a reaction may be increased on a surface in a way additional to that of mass action. In the act of condensation, molecular forces may be brought into play, which raise the chemical potential of the reacting substances; in other words, increase their affinity for each other.

On the whole, the following view is that which is most in agreement with the facts. Owing to the nature of its surface, each variety of enzyme has the property of causing condensation thereon of the components of a particular system. This results in a greatly increased rate of attainment of the natural equilibrium of that system; whether merely by mass action or by increase of chemical potential in addition, is as yet uncertain.

\section{Changes in Carbohydrates}

We may now return to the fate of starch after being subjected to the action of an enzyme in the mouth, which brings about its ultimate conversion to glucose (E., p. 193). The enzyme in question is called amylase, because it acts on starch (amylum). This action, although a rapid one, has not time to be completed, and does not progress further than the formation of some compounds intermediate between starch and glucose, namely, dextrin, 
which is a condensation of several molecules of glucose, but less than the number contained in the molecule of starch, together with the sugar called maltose, consisting of two molecules of glucose united by the elimination of $\mathrm{H}_{2} \mathrm{O}$, and is therefore one of the di-saccharides, or more correctly, bi-hexoses.

After the food is swallowed, it passes quickly down the cesophagus, or gullet, and reaches the stomach. This is a large bag in the course of the alimentary canal, and has muscular rings at both ends, so that the food is not allowed to leave it until it has been thoroughly exposed to the action of the digestive juice secreted by the lining cells. This "gastric juice" is strongly acid, and stops the further activity of amylase as soon as the mass in the stomach has become penetrated by the acid. But this does not take place very quickly, so that the salivary amylase continues its action for some further time. The digestion of starch is, however, not completed by the time that the food is passed on to the small intestine, a long tube in which a variety of processes takes place. Although the stomach is an important organ for the digestion of proteins, as we shall see presently, the process is incomplete, even in this case. In fact, there is no absorption into the blood of any digestive products from the stomach. They are not yet in the state required by the tissue cells.

Maltose, dextrin, and unaltered starch pass, then, into the small intestine, and are next subjected to the action of the pancreatic juice, a mixture of powerful enzymes, one of them being an amylase which completes the digestion of starch and dextrin, certainly as far as maltose and probably, to some extent, as far as glucose. Maltose is acted on by an enzyme, maltase, which completes the hydrolysis to glucose. This enzyme is produced by the cells lining the intestine. Pancreatic juice is a liquid formed by a secreting gland by the side of the intestine, and poured into the intestine by a duct.

The glucose so formed passes by diffusion into the blood vessels, with which the wall of the intestine is richly supplied. It is carried by the blood to the liver, through which it passes before reaching the rest of the body.

Since the whole of the glucose produced by the digestion of a particular meal is not wanted for use at once, a part of it is stored in the liver, and also in the muscles, in the form of an insoluble starch-like substance called "glycogen." The liver itself contains an amylase, which changes the stored glycogen into sugar when required. Moreover, what we have learned about the reversible action of enzymes tells us that this same enzyme is able to form glycogen from sugar, when the conditions are favourable. Since the equilibrium position in such cases depends 
upon the relative concentration of the reagents, it will readily be seen that if one of them is insoluble, it is deposited out of solution as fast as it is produced, and takes no further part in the equilibrium. The result of this is that more of it continues to be formed in order to supply that constituent of the system necessary for equilibrium. In this way, even if the equilibrium position is such that a very small amount of the synthetic product suffices to maintain it, yet a notable quantity may be formed even in a moderate time, if it is removed from the reacting system in any way. Thus it may be removed by its insolubility, or by combining with some other substance, or again by being carried away by diffusion into the blood stream.

Similar considerations apply to the deposition of starch from the sugar formed in the photo-chemical assimilation of carbon in the leaf of the green plant, and to its removal to other parts of the plant, stem, or root during darkness.

But the capacity of the liver and muscles to store glycogen is somewhat limited, and there is another way in which sugar not needed for use at once is stored. This is as fat. The fact is well known to those who grow cattle or pigs for food, although the chemical changes involved are unknown, except in a general way (P., p. 278). A similar change occurs in plants, as shown by fatty seeds, such as linseed.

Although we have hitherto spoken only of starch, it is familiar to the reader that all our carbohydrate food is not in this form. We take cane sugar, maltose, and lactose (milk sugar), which are compounds of two molecules of simple hexoses. These must be split by hydrolysis before they are of use to the cells. We have seen that there is a maltase in the intestine, and appropriate enzymes are also produced there for the other two sugars. Invertase causes the rapid hydrolysis of cane sugar to glucose and fructose; lactase hydrolyses milk sugar to glucose and galactose.

The final destiny of all these sugars is to be burned up to give energy. This takes place chiefly in the muscles, because, as we shall see later, this is the situation where most supply of energy is wanted. The machinery by which this combustion is effected is not completely known, but there is reason to believe that the sugar does not become chemically combined with the actual protoplasmic molecules. We may call to mind that, in a petrol motor, the fuel does not become a constituent part of the mechanism, but is burned up in such a way in relation to this mechanism that its energy becomes available for use.

Although this is what finally happens to glucose and other foods used for energy purposes, there are a number of intermediate products formed before they become carbon dioxide and water. 
Many of these are of importance, because they play a part in various chemical reactions in the cell, and in the abnormal changes of disease. Lactic acid is one of the most important intermediate

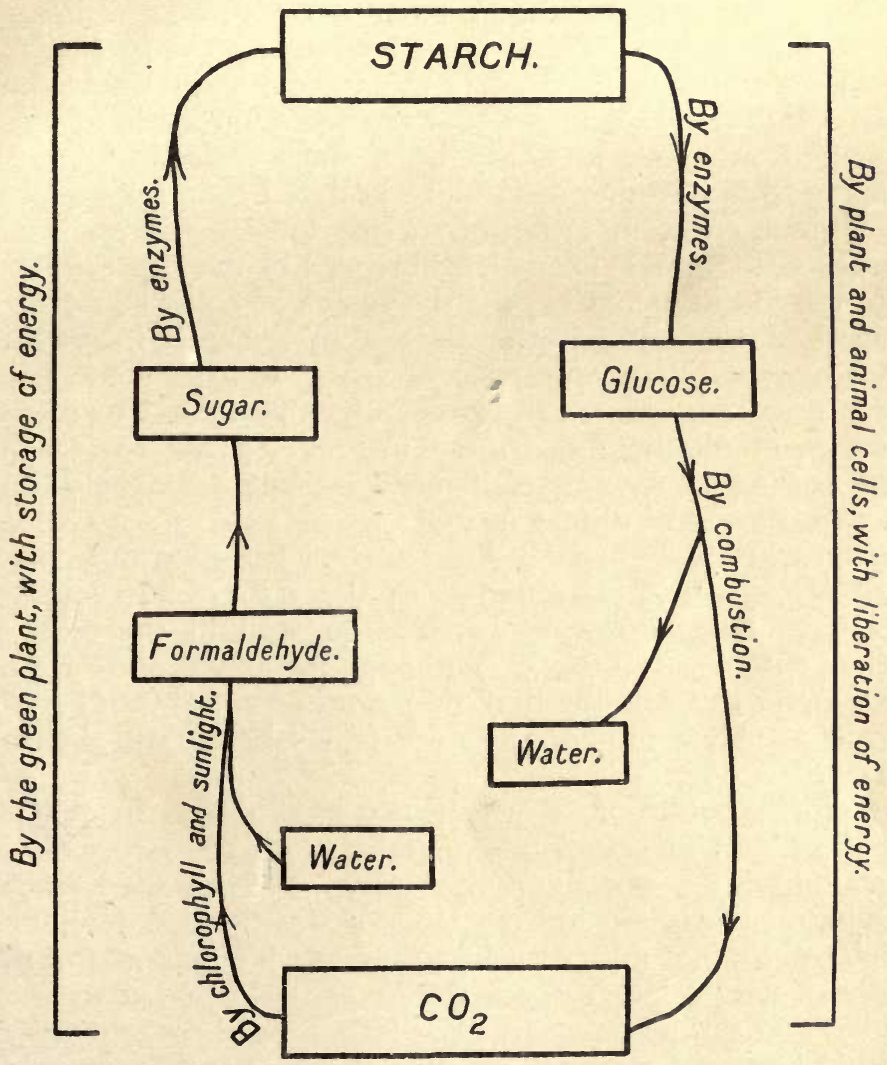

FIG. I.-Diagram of the Carbohydrate Cycle from Carbon Dioxide through Starch to Carbon Dioxide again.

products of carbohydrates on account of its relation to muscle, as will be seen later.

In Fig. I a diagram is given of the carbohydrate cycle.

\section{Fat}

We have already learned something of the chemical nature of fats as esters of glycerol and, incidentally, the fact that there is an 
enzyme that accelerates the hydrolysis and synthesis of esters. This enzyme is known as "lipase."

It is not found in any appreciable quantity in the saliva or in the gastric juice. The first cliange that takes place is when the pancreatic juice is met with. This contains a powerful lipase, and effects the hydrolysis of fats into glycerol and the particular fatty acid. These higher fatty acids are insoluble in water, but are dissolved by the bile. This liquid is a secretion made by the liver, and poured into the intestine by a duct close to that of the pancreas. Bile contains salts of two related complex acids, which have a great effect in lowering surface tension. From what has been said in the previous chapter in regard to the effect of lowering surface tension in increasing the dispersion of colloids, we shall not be surprised to find that the activity of lipase is increased in a marked degree by the presence of bile. Indeed, some kinds of lipase appear to be practically inert in its absence. The increase in activity is, no doubt, due to the increase in the surface of the enzyme, owing to its greater dispersion; but it may also cause a greater dispersion of the fat itself.

Fatty acids and glycerol are absorbed together and, somewhat remarkably, at once recombined in the wall of the intestine to the original neutral fats, which can be seen in the cells of the intestinal surface (E., p. 195). Although the actual proof is difficult, there can be little doubt that this synthesis is effected by lipase, acting in a system in which the water has been reduced by some means.

The fine globules of fat which then pass out of the cells do not enter the blood at once, but are taken into a space which is in connection with a system of branching vessels, called lymphatics. These have their origin in the spaces of the tissues of all kinds of organs, and finally unite in a large vessel which enters the veins at the root of the ncck. The fat thus enters the blood in very fine globules, and can be detected there after a meal containing fat.

What fat is not required for current use as a source of energy is stored up in subcutaneous and other connective tissue in numerous parts of the body. This is the special advantage it possesses over other kinds of food, since the capacity of storage is practically unlimited.

Like carbohydrate, there are intermediate products formed before fat is completely oxidised to carbon dioxide and water. The process is not completely understood, and involves more chemical details than can be given here. 


\section{Proteins}

We have learned that these compounds are the form in which our nitrogen supply is provided. They also serve as a source of energy.

In order that the tissue cells may be able to make use of these substances, they must first be split up into their constituent aminoacids. The first step is taken in the stomach, where the gastric juice contains an enzyme, pepsin, which acts only in a fairly acid medium. The acid present in the stomach is hydrochloric acid, which is secreted by the glands in the walls of the stomach, but not by the same cells that produce the pepsin. Pepsin does not, however, carry the hydrolysis as far as amino-acids, but only to certain polypeptides, called peptones, which still consist of several aminoacid molecules. Peptones are not absorbed in the stomach, but are passed on to the small intestine where the "trypsin" of the pancreatic juice converts them almost entirely into amino-acids, although some of the dipeptides, formed near the end, are difficult to hydrolyse. The operation is completed by another enzyme, "erepsin," which is produced by the cells lining the intestine. Trypsin acts in a faintly alkaline solution, resulting from the mixture of the acid products from the stomach with the alkaline pancreatic juice (E., p. 193).

Although we can only follow the series of changes in the vertebrates, there are indications that the process is common to all animals, even including the amœba. There is a preliminary action of an enzyme acting in acid solution, followed by another in faintly alkaline solution. Since trypsin can act upon the original protein, it is not quite clear why there is a preliminary action by pepsin. It is doubtless a means of hastening the process, because the products of gastric digestion are more rapidly hydrolysed by trypsin than if the process had not already been partially performed.

The amino-acids thus formed are absorbed by the blood vessels of the small intestine, and carried to the liver. The greater part are subjected there to a chemical change, which will be described presently. The smaller part passes on to the tissue cells, which select the particular amino-acids which they require for the repair of their structure, or for growing new structures.

We have already seen that proteins can be burned and used as sources of energy. The process, however, as it takes place in the living organism, is incomplete. The $\mathrm{NH}_{2}$ groups are not oxidised, and are lost as urea. How are these groups split off from the amino-acids? So far as we know, what takes place is as follows. There is an enzyme or enzymes in the liver which 
removes the $\mathrm{NH}_{2}$ group and the hydrogen from their combination with the carbon atom next the carboxyl group (the $a$-position), and replaces them by oxygen, thus forming a ketonic acid. In the same process the carboxyl is reduced to an aldehyde group. Taking alanine ( $a$-amino-propionic acid) as an example :-

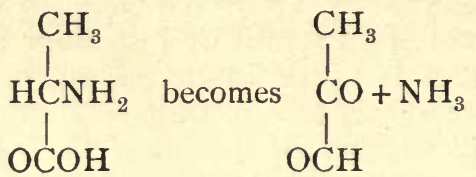

A ketonic aldehyde is produced, in this case pyruvic aldehyde corresponding to the particular amino-acid affected by the process. known as "deamination." Further changes are produced in these compounds by one of three kinds of reaction, under the control of enzymes. Most of those enzymes with which we have been concerned so far have been those dealing with the removal or addition of the elements of water. If a ketonic aldehyde is hydrolysed, we obtain the corresponding hydroxy-acid. In our example it would be lactic acid:-<smiles>CC(=O)OC(C)C(=O)O</smiles>

But there are also enzymes, as we shall see later, which bring about oxidation or reduction. In the first case we get :-<smiles>CC(=O)C(=O)OC(C)(C=O)C(=O)O</smiles>

In the latter case we have :-<smiles>CC(=O)CC(C)C(=O)O</smiles>

Any one of these three acids, passing on to the tissues is oxidised to carbon dioxide and water, giving off energy.

The ammonia from the deamination reaction combines first with carbon dioxide to form the carbonate, which is then converted into 
of bacteria. In the course of the changes brought about by these organisms, intermediate products, such as glucose from the cellulose of the plant tissues, are formed, and these are to a large extent absorbed before they have undergone further destruction.

\section{Movements of the Alimentary Canal}

The various digestive enzymes, whose action has been briefly described above, are formed either by cells lining little pits on the wall of the cavities or by separate organs called glands, which pour in their products by means of ducts. The process of formation of these juices is known as "secretion," to which we shall return presently.

But it will be clear that the effective mixing of these secretions with the food requires a process similar to the kneading of dough to make bread. Moreover, the absorption of the digested material needs the bringing into contact with the wall of the intestine of all parts of the mass in turn. And again, the useless indigestible portion must be carried along to make room for the new material arriving from the stomach, while the new material itself needs exposing to the action of different parts in turn.

The mechanism by which these things are done will be better understood after the later portions of this book have been read. The process is a complex one, involving nervous and muscular factors. It will be best described in its essential features by taking the case of the small intestine, where the simple tubular form shows it in a way most easily followed.

Let us first see what actually happens, and then attempt to explain how it is brought about. We will suppose that an animal has been fed with a meal containing an insoluble powder which is opaque to X-rays, such as bismuth subnitrate. The shadows of the food masses can then be observed on a fluorescent screen in the usual way and their movements followed. Looking at the shadow of a loop which happens for a moment to be at rest, we notice that it is filled with a long column of regular diameter like a sausage. Presently this column is nipped together in several places at the same time, and divided up into a number of small portions. Each of these is next divided up and the parts forced in both directions, so as to join with similar parts of neighbouring masses. The process is repeated many times, and then a powerful nip starts at the end of the mass next the stomach, forcing the whole onwards into the intestine beyond, which becomes lax in order to receive it. This nipping passes along the mass from end to end and empties the loop, much as if it had been seized by the finger and thumb, and the contents pressed out by sliding the finger 
and thumb along. This last kind of movement is known as "peristaltic contraction."

Now as to the way in which these movements are produced. It must be assumed that the reader is aware that there are structures called muscles, which have the power of shortening when required. If arranged in the form of a ring, the ring must be narrowed when the muscle shortens, or even closed up entirely. The wall of the intestine consists in part of muscular tissue, which can, by shortening at particular points, empty out the contents of the intestine at these points into the adjoining part. Further, there are two kinds of muscular tissue found in the body, one kind forming the muscles most familiar to us in our arms and legs, and so on. These are at rest, until ordered to activity by the brain (E., p. 195); the other kind, which forms a large part of the walls of hollow organs and tubes, such as the heart, intestine, and blood vessels are, when left to theniselves, in a state of moderate activity, which shows itself in two ways: It may be present as a steady, uninterrupted state of "contraction," as the shortening of muscle is usually, but incorrectly, called. This state is, as a rule, only partial, since it can either increase or decrease. Or the spontaneous activity may be shown by a series of contractions and relaxations, following one another at regular intervals. The intestinal muscle, when removed from any connection with the nervous system, manifests both these properties at the same time. The series of contractions starts at a point and passes as a wave from point to point along the course of the intestine.

It is clear that before any ordered movements, such as those decribed as part of the digestive mechanism, can take place, a means of control of spontaneous activities must be arranged. It is, in point of fact, usual to find that muscular tissue of this kind, which is known by various names, "smooth," "non-striated," or "involuntary," is under the influence of two kinds of nerves, one of which restrains its activity, the other increases it. We do not know yet how the two effects are actually produced, except that they are due to the different ways in which these two kinds of nerve fibres end in the muscle cell itself, not to any peculiarity of the nerve fibre itself or the process in it. The restraining nerves are called "inhibitory," these increasing activity, "excitatory." In the case of the intestine, the excitatory nerve, a part of that nerve known as the vagus, has also inhibitory functions of a certain kind. Its fibres are not connected directly with the muscle fibres, but with a layer of nerve fibres and cells in the wall of the intestine. Secondary fibres are supplied to the muscle itself from this layer. Now we saw that when the digestive process at one place has gone on for a certain time, the backward and forward movements cease 
and a wave of contraction passes along, preceded by a wave of relaxation. This peristaltic wave occurs also in the intestine after removal from the body, if distended locally in any way. The most satisfactory way of explaining the fact, which has been called the "law of the intestine," is that the nerve cells with which the vagus fibres join give off, each one, two nerve fibres, one of which goes backwards to supply muscle nearer the stomach, and is excitatory; the other goes forward, and is inhibitory. When, therefore, something happens in the interior of the intestine opposite one of these cells, of such a nature as to set this cell into activity, a contraction will be started behind the place in question and a relaxation in front, both these processes travelling onwards as the food mass is pushed between them. We must suppose that something, perhaps of a chemical nature, happens at a certain stage of digestion, and that the movement is brought about which sends the mass onwards.

One of the properties of smooth muscle is that it is stimulated to contraction by being stretched. We understand, therefore, how the distension of an intestinal loop by food passing into it will result in contraction, at this place, and that when a contraction occurs over the food mass, this will be forced into a neighbouring region and in turn cause contraction here. But it is not quite so easy to explain why a number of points along a column of food material enter into activity at the same time, as we saw happens. It is possible that small differences in degree of distension may determine where a contraction is to take place. But we must also remember that any of the activities described can be stopped by the inhibitory nerve fibres in order that a different kind of activity may take its place. These fibres are contained in the nerves called "splanchnic," which issue from the central nervous system, so that it is not impossible that there may be control of co-ordinate movements by the higher centres in response to messages received. The inhibitory fibres in the splanchnic nerves are readily set into activity by stimulation of nerve fibres in the intestine which carry messages to the nerve centres, producing what we shall learn later is a "reflex action." Thus, injury to the intestine, as by gun-shot or shell wound, results in a long-lasting cessation of movement, a reflex "paralysis" of the muscular wall, clearly a beneficial prccess, especially if the injury has resulted in perforation of the wall of the intestine.

The feeling of hunger, as distinct from appetite, is caused by contractions of the empty stomach, by which nervous structures are pressed upon and stimulated.

Appetite, on the other hand, may be described as pleasure anticipated from the taste of food, and may, of course, be present, independently of hunger or actual need for food. It is, however, 
a powerful stimulus to the secretion of the gastric juice, which is, therefore, already present in the stomach when the food arrives there.

\section{Secretion}

The digestive juices, as pointed out, are formed by the cells of organs called "glands," specially devoted to this purpose. Like all the other cells of the organism, these cells derive from the blood the material from which they manufacture their special products. These products are not present in the blood, whose composition we shall have to discuss in other places. While all cells form their own structure, some of them prepare also things for the use of other parts of the body, giving them off by means of a current of water, which washes them out, as it were. The production of this current of water is one of the problems concerning secretion, to which we shall have to give attention.

As will have been evident from what has been said about the process of digestion, glands are not always in action. If we examine under the microscope the condition of secreting cells after a period of rest, we notice that they are filled with a number of granules, differing in kind according to the nature of the gland (E., p. 196). These granules are clearly the material out of which the constituents of the secretion, or some of them, are produced when the gland is excited to activity, because they disappear to a greater or less extent during activity. When the stimulus to secretion ceases, new granules are formed by the cells automatically and, as it appears, by a chemical process of the nature of a balanced reaction, because, when the cell has stored a certain quantity, the formation ceases, the gland being then ready for renewed activity. This stage of preparation requires the expenditure of energy, as shown by the fact that there is an increased consumption of oxygen for some minutes after secretion has ceased. During continued activity, the using up and production of new material keep pace with one another, unless very great demand is made on the gland. In the latter case, the granules are used up faster than they are formed, and they disappear first of all from that part of the cell furthest from the side in connection with the duct.

Before we pass on to discuss what happens in the cell when it enters into activity, we may spend a little time on the ways in which a gland is excited to secrete.

Some glands are supplied with nerves, and when these nerves are stimulated, changes are produced in the cells leading to a flow of liquid. This mode of setting into activity is particularly evident in the salivary glands, and is also present in the gastric glands. The nerves are stimulated by reflex action from various nerves of 
sense. Thus, not only is the taste of food effective, but also the sight and smell. Hence, as we saw, the importance of the possession of appetite.

But in the case of the stomach we find that another mode of stimulation begins to show itself. Glands can be excited by chemical agents, as we know from the fact that there are drugs which have the property of causing them to secrete. Such a drug is pilocarpine. In the course of digestion, certain substances are produced by the action of the contents of the alimentary canal on the cells of its walls. These substances are absorbed into the blood and arrive in this way at some gland which is sensitive to them. The most obvious of these mechanisms is that of the pancreas. When the acid contents of the stomach pass into the small intestine, they cause the formation in the cells of a substance which has been called "secretin," but whose chemical nature is still unknown. This passes into the blood, and arriving at the pancreas, excites this organ to secrete. It is a remarkable fact that the trypsin contained in the pancreatic juice is inactive until it has been acted upon by another substance, "enterokinase," apparently an enzyme, secreted by the walls of the intestine.

So far as can be made out, what happens in the secreting cell when stimulated is somewhat as follows. We have seen that the cell at rest contains a store of material, which has been produced by the expenditure of energy. Its molecules, or some of them, are large ones, and onset of activity is associated with a splitting up of these molecules into smaller ones. The consequence of this is that the osmotic pressure of the cell contents rises, water is absorbed from the blood and, supposing that the cell membrane is impermeable to these solutes, the cell merely becomes distended. But now, supposing that the end in relation with the duct becomes permeable, then the pressure will cause a flow of liquid into the duct, carrying with it in solution the constituents of the secretion. Sometimes granules appear to escape bodily from the cells, becoming dissolved in the ducts later. This process will continue as long as any osmotically active material is present in the cell and the membrane at the duct end remains permeable. It can be imitated in a model (E., p. 197).

In some secreting mechanisms of plants it can be shown that changes of permeability occur at the end of the cell at which the liquid appears. A similar state of affairs is at the basis of the root pressure. If the stem is cut through, a flow of liquid, under fair pressure, occurs from the cut end, being absorbed from the soil.

In the animal cell, proofs of increased permeability in activity are more indirect. The cells take up certain dyes more readily. 
But the most interesting phenomenon showing a change of permeability is the electrical change which takes place in activity. We may spend a short time on this, since the explanation applies to muscle and nerve as well as to secreting glands.

We saw above that a membrane may be impermeable to an electrolyte because one of the ions of this electrolyte cannot pass through, although the membrane may be easily permeable to the other ion. This opposite ion goes out only as far as the attraction of the oppositely charged ion inside the membrane will allow it. We have here the Helmholtz double layer in one of its forms. A simple illustration may enable this important conception to be grasped. Imagine two large pastures separated by a fence, and that the spaces between the bars of this fence are wide enough to allow lambs to get through, but too narrow for their mothers.

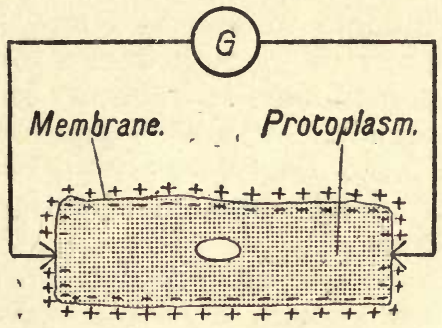

(A)

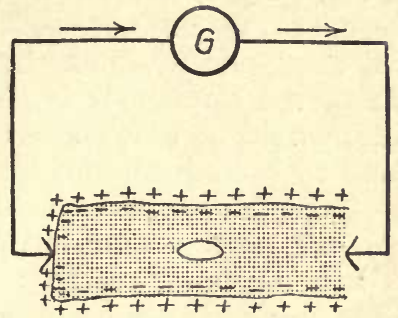

(B)

FIG. 3.-Diagram of the electrical state of the resting cell (A) and that of an active cell (B).

Introduce into one of these pastures a flock of sheep, each ewe with one lamb. In the course of their wanderings they will arrive at the fence. The propensity of the lambs to wander further will take them through the fence, but the ewes must remain behind. However, the attractive forces of their mothers, especially that of food, will prevent the lambs from being in any number far from the fence at any time. Similarly, the presence of the lambs in the adjoining field will prevent the ewes from wandering far from the fence. It may be said that the thickness of the layer would be somewhat great, but if we imagine molecules magnified to the size of sheep the proportions would not greatly differ from the molecular one.

Let us suppose that the membrane of a particular cell is permeable to the cations of some salt contained within the cell, impermeable to the anions of this salt. The cell will be surrounded by an electrically positive sheath, the other component of the double layer with a negative charge being on the inside of the membrane. Fig. 3 shows the arrangement at A. If we connect 
the two ends to a galvanometer, we should not be able to detect any electrical difference between them; any two points on the surface are at the same potential. Imagine next that one end loses its semi-permeable nature, as in B, so that the two kinds of ions freely mix by diffusion. This end will become neutral, and merely serve as a conductor to the internal member of the double layer. Accordingly, we observe a deflection of the galvanometer. At the same time the way is open for the escape of solutes from the cell. It will be clear that another membrane might be permeable to anions only. Or again, during activity a reversal of the sign of the permeability might occur. There are, indeed, many possibilities. The fact of the occurrence of an electrical change in gland cells on activity is referred to here as one of the pieces of evidence in favour of an increased permeability (E., p. 197).

We saw in our experiments on blood corpuscles that the osmotic pressure of the salts in blood amounts to several atmospheres. Now if the liquid produced by the secreting glands were a solution merely of the important organic constituents in water, its osmotic pressure would be low, and a large amount of work would have to be done to hold back the salts. Accordingly, we always find the salts of the blood present in secretions, although sometimes in lower concentration than in the blood.

It is to be remembered that secretions are produced in other situations besides those in connection with digestive processes. We have the sweat glands of the skin, for example, for the purpose of getting rid of excess heat by evaporation of water. Further, the secretion of some organs does not flow away in a duct, but the products diffuse into the blood and exert an action on other organs by this means. These are called "internal secretions" or "hormones," and will be dealt with later.

Thirst.-This feeling is due to dryness of the throat, owing to deficient secretion of saliva. When the blood has lost water, its osmotic pressure rises, consequently the cells of the secreting glands have to do more work to extract the watery saliva from it; or the expenditure of the same amount of energy results in less secretion. The body is continually losing water from the lungs, skin, and kidneys; unless this is replaced, the blood becomes concentrated. The osmotic pressure of the blood is higher than that of saliva, hence the natural direction of flow of water would be from duct to blood vessels. To counteract this, the expenditure of energy is required.

\section{Respiration}

In order that energy may be obtained from food materials, they must be burned or oxidised by combination with oxygen. 
Thus it is not, strictly speaking, correct to refer to the energy value of fat, for example. The system possessing the potential energy is fat plus oxygen.

Animals of small size or of flat form obtain their oxygen by free diffusion to the tissue cells. But as soon as larger dimensions and more complex forms appear, the necessity of special arrangements for conveying oxygen to the tissues becomes evident.

In insects, we find a peculiar branching system of fine tubes, called "tracheae," which contain air and are distributed to all organs (E., p. 200). The air is changed by squeezing movements which press it out through certain openings on the side of the body, while fresh air enters when the pressure is relaxed.

In crustacea, molluscs, and vertebrates, a liquid, the blood, is carried to all parts of the body by a tubular system. The arrangements of this system will be described in a later chapter. For the present, it will suffice to remember that a supply of oxygen is conveyed to all tissues in this way. But oxygen is only slightly soluble in water, and a very copious current of such a solution would be necessary to provide enough oxygen for the vigorous movements of the vertebrates; in fact, so great a current would be required as to be mechanically impossible. Accordingly, we find in the blood certain very small red bodies, the red corpuscles, which contain that remarkable compound, haemoglobin, about whose chemical nature we have already learned some facts. The most important one is that it takes up oxygen when the pressure of this gas is about that which it has in the atmosphere, and gives it up again when the pressure is lower, as in the tissues, where the gas is being continually used up (E., p. 200).

We must at this point understand what is meant by the tension of a gas, since the expression is often to be used. Suppose that we have a mixture of air with carbon dioxide, such that in one hundred volumes of the mixture there are 95 of air and 5 of carbon dioxide. And further, that the mixture of gases is at atmospheric pressure. The five volumes of carbon dioxide are diffused throughout the space, and if we imagine the air removed, it is clear that the pressure would be only five-hundredths of an atmosphere, since the five parts fill the space of one hundred. The pressure of the carbon dioxide in the mixture is therefore five-hundredths of 760 $\mathrm{mm}$. of mercury; that is, $38 \mathrm{~mm}$. This is known as the partial pressure or tension of the carbon dioxide in the mixture. Similarly, the tension of the oxygen, which forms 2 I per cent. of air, will be, in our mixture, $21 / 100 \times 95 / 100 \times 760=151.6 \mathrm{~mm}$. A liquid in contact with such a mixture will dissolve carbon dioxide until the tension of the gas in the liquid is the same as that in the gas phase, 
since there must be as many carbon dioxide molecules leaving the surface of the liquid in a given time as there are molecules entering it; otherwise there could be no equilibrium. But although the tension must be the same whatever the liquid taken, the actual amount of carbon dioxide dissolved may vary greatly. Thus, water dissolves more than a strong salt solution does. Further, the amount of different gases dissolved by water varies greatly at the same tension.

If now we take a solution of haemoglobin and expose it to the air, that is, to $160 \mathrm{~mm}$. tension of oxygen, we find that it takes up a much larger amount of oxygen than water does. Next, expose it to a vacuum or an atmosphere of pure nitrogen, that is, to a zero tension of oxygen; the oxygen which it contains is given off again. What happens, however; if we expose it to a tension of oxygen of one-eighth of that in the atmosphere, namely, to 20 $\mathrm{mm}$.? We find that it takes up oxygen, but less than at $160 \mathrm{~mm}$. Moreover, at all tensions between $O$ and $100 \mathrm{~mm}$. the amount of oxygen taken up has a definite value; and, if a graph be made, it will be seen that these values are not directly proportional to the tension, but the curve rises more steeply at the lower tensions, so that very little more is taken up at $100 \mathrm{~mm}$. than at $80 \mathrm{~mm}$., while above $100 \mathrm{~mm}$. haemoglobin is practically saturated with oxygen, a further rise in tension resulting in no further amount being taken up.

Although this union of oxygen with haemoglobin is usually looked upon as a chemical compound, it must not be overlooked that there are difficulties in this view, and that it is held by some that the case is more analogous to the adsorption of gases by surfaces such as that of charcoal, which played so great a part in the protection from poison gases in the War. The question is not yet decided, and more complete discussion may be read elsewhere (P., pp. 613-625). But there are some facts of interest that may be mentioned briefly here on account of their interest and importance.

We saw above that when haemoglobin has taken up a certain amount of oxygen, any further rise in tension does not result in any more being taken up. In speaking of the chemical nature of haemoglobin, it was pointed out that each molecule contains one atom of iron. Hence, by comparing the amount of oxygen taken up in saturation with the amount of iron, it is possible to find out the number of molecules of oxygen that unite with a molecule of haemoglobin. It is found to be precisely one molecule. This is a fact strongly indicating a definite chemical compound. But a difficulty arises at once as to the nature of the compounds present when the haemoglobin is only partially saturated. It 
should be kept in mind that haemoglobin is in colloidal solution in water, and about 5 per cent. is the most that can be dissolved. The red corpuscles contain 35 per cent. of haemoglobin, and 63 per cent. of water, so that the haemoglobin must be in the form of a moist solid. The following considerations will show that if oxy-haemoglobin (as the oxygenated form is called) is a definite chemical compound, it is unique. There are various compounds which give off oxygen or carbon dioxide at a certain tension of these gases, and combine with it again at a higher tension. But the point is that there is no half-way state. Above a particular "dissociation tension," according to temperature, the whole is in the form of the complcte compound. Just below this tension the wohole of the gas is given off. It may be stated to be a case of "all or nothing." To get over the difficulty, it has been suggested that there is a series of compounds of haemoglobin with oxygen of the composition $\mathrm{HbO}_{2}, \mathrm{Hb}_{2} \mathrm{O}_{4}, \mathrm{Hb}_{3} \mathrm{O}_{6}$, etc. These would each obey a different form of the law, deduced from mass action, of the rate of combination in relation to concentration of oxygen, that is, the tension of oxygen. Of course, the difficulty is not present in such a case as that of adsorption by charcoal, where the amount condensed on the surface is in proportion to the tension of the gas, up to the point of saturation.

Again, it is found that the amount of oxygen taken up by haemoglobin is less at a higher than at a lower temperature, although the rate at which it takes it up or gives it off is greater at the higher temperature. This is one of the peculiarities of adsorption also, owing to the negative temperature coefficient of surface tension. It might be supposed to imply a disadvantage on the part of warm-blooded animals, but it seems to be more important to obtain the oxygen quickly than to have the larger reserve, which is made up for by rapid replacement of the blood by vigorous circulation.

Haemoglobin, being colloidal, is subject to aggregation by electrolytes, and the fact shows itself in an effect on the form of the dissociation curve such that when acids or salts are present, less oxygen is taken up at a given tension of the gas. The difference is not great at the higher tensions ( $90-100 \mathrm{~mm}$. of mercury), but marked when it is $15-20 \mathrm{~mm}$.

The darker colour of blood which has lost oxygen is familiar in the appearance of the veins. Although they look bluish, as seen through the skin by reflected light, the colour of the blood itself may be more correctly described as crimson, when compared with the bright scarlet colour of fully oxygenated arterial blood. The scarlet colour in the arteries is due to the fact that the blood has taken up oxygen in the lungs. As it passes through the tissues, 
it becomes crimson as the oxygen is given up to the active cells, which use it for combustion purposes (E., p. 20r).

A fact rather difficult to reconcile with chemical combination is that oxygen is not the only gas or vapour taken up by haemoglobin. Carbon monoxide, nitric oxide, carbon dioxide, and chloroform are absorbed, apparently in a similar way to oxygen, although in different numerical proportions.

The taking up of carbon dioxide by haemoglobin leads, naturally, to the question of the carriage of this gas in the blood, so as to remove it from the tissues, where it is being continually produced by oxidation of carbon compounds. As the arterial blood reaches the tissues, oxy-haemoglobin gives up part of its oxygen, since the tension of oxygen in the cells is low. The tension of carbon dioxide in the cells becoming higher than in the blood, owing to the above-mentioned combustion process, it passes into the blood, and is taken up by the haemoglobin. Experiments show that carbon dioxide drives off a part of the oxygen from the haemoglobin. Or, put in another way, under a given reduced tension of oxygen, less is held by the haemoglobin in the presence of carbon dioxide than in its absence. The advantage of this is clear, since more oxygen is set free for use. We are reminded of a similar state of affairs in the case of enzymes, where one substance can drive another out of adsorption on the surface.

\section{The Lungs}

The blood returning to the heart by way of the veins contains then less oxygen and more carbon dioxide than when it arrives at the tissues. How does it replenish its oxygen and get rid of the excess carbon dioxide? It is sent by the heart to the lungs for this purpose. These organs consist essentially of an elaborate system of little bags full of air, on the walls of which there is a fine network of minute blood vessels (capillaries). The blood in these vessels is separated from the air by a very thin membrane, so that the haemoglobin is quickly exposed to a tension of oxygen high enough to saturate it, and to one of carbon dioxide low enough to remove a great part of the carbon dioxide from it. But there must clearly be some means of renewing the air in the lungs. This is done by alternately expanding and contracting the cavity of the chest, in which the lungs are contained, a process known as breathing. There are muscles which raise the ribs from an oblique to a more horizontal position, and there is a muscular partition between the chest and the abdomen, the diaphragm, which at rest is in the form of a dome projecting into the chest. When it contracts, the top is pulled towards the abdomen, since the lower edge is fixed 
to the ribs and spinal column. By these two means air is sucked into the lungs. When the contraction of the muscles ceases, the chest returns to its position of rest, expelling part of the air contained in it. Under vigorous respiration, however, there are muscular movements which assist in pressing air out. The whole of the air is, of course, not driven out, so that in the depths of the small air sacs, where the exchange between blood and air is effected, the tension of oxygen is not as high as in the atmosphere, although it is high enough to saturate the haemoglobin; while the tension of carbon dioxide is not so low as in the outer air, but is lower than in the venous blood.

The way in which the supply of air by respiratory movements is regulated in accordance with the needs of the organism is by the fact that the nerve centre, which is responsible for sending periodic discharges to the muscles acting on the chest, is extremely sensitive to a slight rise in the hydrogen-ion concentration of the blood. When more oxygen is being consumed by the activity of cells, more carbon dioxide is being given off. This becomes an acid when dissolved in water, and raises the hydrogen-ion concentration of the blood to a slight degree. The respiratory centre is stimulated, more oxygen is supplied, and the excess of carbon dioxide removed (E., p. 202). It is important to remember that the production of any acid by the tissues has the same effect. Since acids are produced in the course of the normal metabolism of fats and carbohydrates, and subsequently oxidised, it is clear why they appear in the blood in states where there is deficient supply of oxygen, as in lowered rates of circulation of blood, and so on. A rise in hydrogen-ion concentration of the blood results, owing to the fact that these acids drive off carbon dioxide from the bicarbonates contained therein. This risê stimulates the respiratory centre, and tends to automatic benefit by a more copious supply of oxygen. It will also be clear that treatment of this so-called "acidosis" by giving alkalies is inappropriate, because the stimulation of the respiratory centre is thereby prevented, and spontaneous supply of the necessary increase in oxygen is retarded.

\section{The Mechanism of Oxidation}

The supply of oxygen to the tissues is provided by the means described. But the mere presence of ordinary oxygen is not sufficient, as will be evident when it is remembered that glucose is one of the foods most largely burned for the supply of energy, and that glucose is not oxidised by the air, or so slowly as to be useless for the purpose in view. What we need is to raise the chemical potential of the oxygen so that it shall attack substances refractory 
to it in its ordinary form. Although this can be done in various ways, we do not as yet understand completely what is the change that takes place. Sir W. Ramsay taught that its activity was manifested during the change from the quadrivalent to the bivalent form, that is, in the process of losing electrical charge. It is interesting to connect this view with what was pointed out previously in our general discussion of the energetics of living organisms, namely, that it is in the process of transfer of energy that those activities which we recognise especially as manifestations of life are to be found.

The way in which the "activation" of oxygen takes place in the living cell may be described briefly, as follows.

Although most of the materials oxidised in the cell are refractory to ordinary oxygen, certain constituents are slowly oxidised by it. Such are the unsaturated fats and lipines. These are said to undergo a process of "autoxidation" (E., p. 202). Now, in this process, investigation has shown that a rather curious thing happens. When a part of the substance is oxidised to a simple oxide, energy is given off, as in the ordinary process of combustion. But this energy is not entirely lost as heat in the case of autoxidation. Simultaneously with the oxidation of one molecule to a lower oxide, another one is converted to a peroxide, which requires a supply of energy to put in the extra atom of oxygen. Peroxides have higher powers of oxidation than ordinary oxygen has; they supply oxygen to oxidisable substances at a higher potential than it possesses in its ordinary molecular form.

A rough idea of this process of raising chemical potential may be obtained by thinking of the increased destructive effect of a weight when dropped from a greater height. To this greater height it must have been raised by the doing of work upon it.

But even peroxides are not powerful enough to oxidise sugar or lactic acid. Hydrogen peroxide does not cause the evolution of carbon dioxide from lactic acid (E., p. 203). There is, however, a catalytic means by which hydrogen peroxide and similar peroxides can be made to afford oxygen at a higher potential. The addition of a trace of a ferrous salt (Fenton's reaction) results in the complete oxidation of lactic acid to carbon dioxide and water (E., p. 203). What the exact mechanism of this reaction is, has not been completely explained.

We may ask, has there been found in the living cell any agent similar in action to that of the iron salt in the above reaction? In the following description, when certain enzymes are stated to be obtained from particular sources, it is not to be understood that they are only present there, but that from this source they 
can conveniently be prepared free from admixtures which obscure their typical action.

A preparation can be obtained from the root of the horseradish and elsewhere which has the same action on hydrogen peroxide as ferrous salts have (E., p. 203). That is, it enables the peroxide to oxidise lactic acid, etc. It has been called pcroxidase, having the general characters of an enzyme. Its action on the peroxide is quite different from that of catalase, another enzyme of very wide occurrence. While the latter causes the evolution of gaseous oxygen, and does not increase the oxidation potential of the peroxide, peroxidase causes no evolution of oxygen, but has a marked effect in raising the oxidative power. Although the composition of peroxidases has not yet been definitely established, there is evidence that they consist essentially of the colloidal hydroxide of a metal, such as iron, copper, or manganese, which is capable of existence in two forms, one produced from the other by an oxidation. In the actual enzyme these hydroxides are associated with some stable organic colloid-gum, protein, etc. (P., p. 585). The function of this colloid appears to be to protect the hydroxide from aggregation and loss of active surface by the effect of electrolytes.

Thus, the concurrence of four factors is required-(I) oxygen ; (2) an autoxidisable substance; (3) a peroxide, produced by the action of the first on the second; and (4) a peroxidase. In many cases we can separate from cells complexes containing peroxides and peroxidases; these are often called oxydases.

A convenient reagent in the investigation of such systems is an acid contained in the gum-resin, guaiacum. This is oxidised by active oxygen, not by ordinary oxygen, or only very slowly. When oxidised, a blue pigment is formed. No effect is produced by a peroxidase alone, nor by an organic peroxide alone; only when combined. If placed on the cut surface of a potato, a blue colour is produced. Hence there must be both peroxide and peroxidase present. In some cases a substance is naturally present which changes in colour when oxidised. This is the origin of the brown tint seen to form on the cut surface of a living apple. When the cells are cut across, and exposed to oxygen, an autoxidisable substance gives rise to a peroxide, which is then acted upon by a peroxidase. The active oxygen, thus available, oxidises a colourless compound, also present in the cells, forming a brown pigment (E., p. 203).

There are many subsidiary details concerning these oxidation mechanisms for which space is not available here. The account given is a brief summary of the state of knowledge at present, which 
has been arrived at after numerous investigations, at first apparently complex and contradictory.

From previous remarks in various places of this book, it will be clear that reduction processes also play an important part in cell life. Some of these processes, up to the present, have been shown to be under the control of enzymes, and substances similar to aldehydes take the place of peroxides. But the mechanism is still somewhat obscure (P., p. 586). It will be clear that when one substance is reduced, another has to be oxidised in order to take away the oxygen from the first (E., p. 203). 


\section{CHAPTER III}

\section{WORK-THE MUSCLES}

ALTHOUGH movement is not the only way in which the energy of food is used up, it is the most striking and obvious way. It is perfectly clear that work is done when we raise a weight or throw a ball.

On the other hand, it is unnecessary to remind the reader that energy is expended in many other ways, as in the overcoming of osmotic pressure, the formation of chemical compounds of a higher potential than those from which they arise, and so on.

In the present chapter we have to learn something about muscular activity and its mechanism.

Suppose that we have a set of fibres attached at one end to a bone and at the other end to another bone, which is capable of moving by a hinge joint at the end of the former, and that the two bones are placed so as to be in line with one another. It is plain that if the fibres shorten, the two bones will be moved so as to form an angle with one another, since in this position the line joining a point on one to a point on the other is shorter (E., p. 204). In general, the action of a muscle when it enters into activity is to bring closer together the points to which its two ends are attached. One of these points is usually fixed, and is called the "origin" of the muscle; the other is movable, and called the "insertion." But, for special purposes, the parts may be reversed. For example, the arm muscles may move the arm itself when the body is fixed, or they may raise the body when the hands are holding a fixed bar. A very great variety of movements is rendered possible by the numerous muscles and bones connected by joints, found in the vertebrate body.

The first point to notice is that the designation "contraction" is not really a correct one. The muscle does not change in volume, but in shape. It becomes shorter and thicker. The increase in thickness can easily be felt in the biceps muscle on the front of the upper arm.

We know, further, that if we attempt to move a very heavy object our muscles enter into great activity, but are unable to 
change in length unless the object yields to our efforts. The fact becomes obvious when we break a string by pulling it (E., p. 204). Take a piece of fine, non-extensible string and find the weight necessary to break it. Then take another piece and break it by pulling with the muscles. No change in length of the muscles can occur until the string breaks, but a force equal to the weight in the previous experiment must have been exerted on the string in order to break it, and this was done before the string broke and the muscles shortened.

The fact is expressed in the statement that the muscle develops a state of "tension" if not allowed to shorten. This is, indeed, the more fundamental fact, since it is the production of the state of tension that causes the muscle to shorten and to do external work.

If we take a coiled steel spring, hang it vertically and increase its length by pulling upon it, a state of tension is produced in it, and, by virtue of this, if a weight is attached to its lower end and the hand pulling it is removed, the tension of the spring does work by raising the weight (E., p. 204). It is somewhat difficult to realise the state as applied to muscle. If we take a coil of lead wire similar to the steel spring and stretch it to the same length, no tension is developed, because lead has not the elastic properties of steel. It may be said, then, that a muscle, when it "contracts," changes its state from that of a stretched lead coil to that of a stretched steel coil, without necessarily altering its length.

The details of the way in which this happens and the origin of the energy set free belong to one of the most difficult parts of physiological science, and are by no means clear, as yet.

In order that a muscle may be put into a state of activity, we may apply what is called a "stimulus," either to the muscle itself or to the nerve which enters it. The most convenient form of stimulus is an electrical one, since it can be adjusted in strength in an accurate and simple manner (E., p. 205). But other forms of stimulation may be used - a tap, heat, or application of salt.

Let us make what is called a "nerve-muscle preparation" from a frog (E., p. 204). We can make the muscle do work by raising a weight, although, being cut out of the body, it is impossible for it to receive any supply of energy from outside itself. It must, therefore, contain a store of energy within itself, and may be compared to a wound-up clock spring, a raised weight used to drive some mechanism, or again, the cordite charge in a cartridge.

If, by repeated stimulation, we make an isolated muscle perform a long series of contractions, we exhaust its store of potential energy; it becomes "fatigued," in one sense of the word. This store of energy is not replenished under the conditions of our experiment; but we know from experience that a muscle recovers 
when in its natural situation, and is supplied with blood. It is clear that its store of energy is made up again. This is found to be by the oxidation of some material brought to it by the blood. It is also found, experimentally, that the supply of energy obtained in this way follows the act of contraction itself. While there is no consumption of oxygen in the act of contraction itself, nor any carbon dioxide given off, both of these take place in the period following the contraction. That oxygen is not used in the act of contraction itself is readily proved by the fact that a muscle can execute a long series of contractions in an atmosphere of nitrogen.

Some food material is burned, therefore, to supply the potential energy which a muscle has lost in doing work and to prepare it for more work. It appears that glucose is used preferably when available, but that fat or the non-nitrogenous part of protein can be used. The same amount of food energy is used for a given supply of muscle energy in each case. We may note here that the fact that either carbohydrate or fat can be utilised, places a difficulty in interpreting the muscle system as being a chemical one, in the strict sense.

The method by which it is discovered whether carbohydrate or fat is being used in muscular work in any particular case is of interest. Since the former may be looked upon as having all its hydrogen already completely oxidised, all the oxygen used is taken up in oxidising the carbon to carbon dioxide, and the volume of carbon dinxide produced is equal to that of the oxygen taken in. If, then, we determine, during a period of muscular work, how much oxygen is taken in and how much carbon dioxide is given off, and compare the ratio with that before the work, we shall find this ratio increased if a larger proportion of carbohydrate is being burned. If nothing else but carbohydrate is burned the ratio, obviously, is unity. This ratio is known as the "respiratory quotient." On the other hand, fat requires oxygen to burn its hydrogen as well as its carbon, so that the carbon dioxide given off in proportion to the oxygen used is much less than unity, and the respiratory quotient would be low when fat is being burned in the organism.

The only chemical change definitely known to occur in the contractile process itself is the production of lactic acid (E., p. 205). It is clear that this must arise from some source in the muscle, but what this is we do not exactly know. In the second stage, which succeeds the contractile one, and that in which the muscle recovers its energy by the aid of a combustion process, this lactic acid disappears, and there is evidence that it is burned in order to give the energy. Glucose must be taken up in some way in order to afford the lactic acid produced in a subsequent contraction, but it 
is at present impossible to say how the energy produced by the oxidation of lactic acid is stored in the muscle.

Some light is thrown on the nature of the mechanism which causes the characteristic state of tension by two experimental facts :-

I. It is found that the magnitude of the tension developed, and therefore of the work done, is greater the longer the fibres of the muscle are at the moment when the state of tension is brought about. If the muscle is stretched, a more vigorous contraction is obtained. This applies, naturally, only within such limits as not to affect the muscle fibres injuriously (E., p. 206). The fact shows, in the first place, that change of volume of some elements of the structure is not the determining factor in the process, because the volume is not altered by stretching the muscle. In other words, we cannot look for an explanation of the origin of the tension in osmotic forces. What has been increased in the experiments referred to is the length of the fibres and certain constituents in them. This means that there has been an increase in the area of certain surfaces arranged longitudinally. We think at once of that property of boundary surfaces that results in surface tension, and that it is by changes in this surface tension that the state of tension of the muscle, as a whole, is produced. Now, what does the structure of muscle suggest? Examination of the microscopic structure of that kind of muscle with which we are dealing, the voluntary or skeletal muscle, which is under the control of the will and moves parts of the bony skeleton, we find that it is composed of long narrow fibres of a protoplasmic material ("sarcoplasm"), in which are embedded a number of very fine threads of somewhat complicated nature ("fibrillæ") (E., p. 206). We have provision, therefore, for the boundary surface between phases demanded by the surface tension theory. When hydrogen ions make their appearance in consequence of the formation of lactic acid, or other acid, at this contact surface between sarcoplasm and fibrillæ, a change in surface tension results. The surface energy provided by this is in proportion to the area of surface on the fibrillæe or to their length, as found by experiment.

II. The second fact which tends to confirm the view that surface forces are responsible for the tension of muscular contraction, is that the tension developed is higher at a low temperature than at a higher one (E., p. 206). As we saw in an earlier chapter, this negative temperature coefficient is a peculiarity of surface tension, so far as concerns those various phenomena which could play a part in the process.

The fact itself is shown also by the behaviour of smooth muscle, 
such as that of the alimentary canal, whose state of "tonic" contraction is relaxed by warming.

The effect of temperature excludes another explanation which has been suggested, namely, that acid increases the amount of water taken up in the swelling of colloidal structures, and that the arrangement in muscle is such that the swelling causes the shortening of the fibrilla. This imbibition, however, has the usual positive temperature coefficient ; is greater as the temperature rises.

There is yet much to be learned about the intimate nature of the process of muscular contraction, but further discussion would not be profitable here (P., pp. 436-458).

\section{Gradation of Contraction- "All-or-nothing"}

Practical experience teaches us that we can cause our muscles to contract with different degrees of strength. Since any individual muscle consists of a large number of fibres, the adjustment might in theory be made in two ways, either by causing all the fibres to contract, but with less than their maximum force, or by causing only a certain varying number to contract, but each always with the same maximum degree of intensity. If we call to mind the similarity of a muscle to the propelling charge in a cartridge, we realise that the former method is less probable than the latter. Although a certain small expenditure of energy is required to move the trigger, this has no relation to that set free in the explosion of the charge; and whatever the strength with which the trigger is pulled, the energy set free is the same. The movement of the trigger corresponds to the stimulus applied to a muscle, and this has no relation to the energy set free in a contraction. Direct experimental proof, however, shows that the changes in degree of contractile strength in a muscle are actually due to the putting into action of a varying number of individual fibres, each working at its greatest capacity. Of course, this does not mean that a fatigued muscle can exert the same degree of tension as a fresh muscle. It means that, so far as any fibre is concerned, whatever the strength of the stimulus, if it has any result at all, the force of the contraction is the greatest that this fibre can exert in its state at the time.

We shall see later that the same statement applies to any individual nerve fibre, so that it is impossible to vary the strength of the stimulus to a muscle fibre. Thus, even if the latter were capable of different degrees of contraction, there is no means of altering the strength of the normal stimulus so as to make use of the property. In the nerve, as in the muscle, it is a question of "all-or-nothing." As in the muscle, adjustments are made by altering the number of fibres in action. 


\section{Refractory Period}

A word must be said next about another important property of muscle and other excitable tissues. If a second stimulus arrives at a brief interval, a fraction of a second, after a previous one, the second stimulus produces no effect. This interval of time during which the muscle is inaccessible to stimulation is known as the "refractory period," and is exhibited while the muscle is in the initial stages of giving effect to the first stimulus (E., p. 208). If we remember the evidence that an essential part of the process of excitation consists in an increase of permeability of the cell membrane, we see that it cannot be repeated until the membrane has recovered its normal state of semi-permeability.

\section{"Staircase"}

Another interesting phenomenon is that of the "staircase." If a muscle has been at rest for some time, it. will be found that the strength of the contraction increases for each successive stimulus during a few contractions (E., p. 207). It appears that a certain very small degree of acidity is that best adapted for maximum contraction. As we saw, lactic acid is produced in contraction, and a trace is left after each contraction, gradually increasing until it is oxidised as fast as it is formed.

\section{Tetanic and Voluntary Contraction}

The simple form of contraction which follows a single electrical shock lasts an appreciable time, varying with the particular muscle in question. In the frog it lasts about a tenth of a second. Now the refractory period referred to above lasts only something over a thousandth of a second. If, therefore, a second stimulus arrives later than this, but during the time in which the muscle is shorter than at rest, a further shortening takes place, and another stimulus and shortening may be superposed on this. Each succeeding stimulus after the first, however, has somewhat less effect on the length of the muscle than the one before it, so that, after a certain number, the height becomes practically steady, but much higher than that produced by a single stimulus (E., p. 206). This state is known as a "tetanic" contraction, and is similar to that resulting from a normal discharge from the nervous system, which consists of a series of stimuli, varying in number according to the length of time that the muscle is required to remain in contraction. 


\section{Muscular Mechanisms of Various Kinds}

It is not only for the purpose of bringing about effects in the outer world that muscles are made use of. Those concerned with breathing and in speech, and those by which the eyes are moved, may be referred to. The importance of the latter will be seen later.

That kind of muscle called smooth or involuntary has been mentioned already in connection with the movements of hollow organs, such as the alimentary canal, and its general properties have been described. The heart and blood vessels will be dealt with in a subsequent chapter.

\section{Posture Phenomena}

There are some rather remarkable phenomena exhibited, especially by involuntary muscle, but also in a certain way by voluntary muscle. They are not yet completely understood, but are of much importance. If we try to keep a weight raised with the arm outstretched, we soon become aware that a continuous expenditure of energy is required. On the other hand, a bivalve mollusc, such as an oyster, is able to keep its shell firmly closed, even when continually pulled upon by a weight, for a long time without signs of fatigue or evidence of consumption of material. There appears thus to be a possibility for certain muscles to maintain themselves at various lengths, which oppose resistance to stretching, but without the presence of a state of tension. It is as if they had become fixed at a particular length, as by freezing, and that a kind of thawing process was necessary in order to restore them to their original state. We may picture the state as being analogous to the holding up of a weight, after it has been raised to a height by the expenditure of energy, by slipping a support underneath it. It does not fall again until the support is removed. The process of relaxation in the muscle, corresponding to the removal of the support, is brought about by the stimulation of a nerve, and does not take place otherwise; this nerve is a different one from that which induced the shortening. Thus, if certain nerves supplying the closing muscle of the mollusc be cut while the muscle is in a state of contraction, it remains permanently at this length, unless the end of the nerve in connection with the muscle is stimulated, and then relaxation occurs. These properties are exhibited by the urinary bladder of the vertebrate in a striking way. If this organ were like an india-rubber ball, the greater the filling the higher would be the tension of the walls and the pressure inside it. But this is not the case. It may possess very various degrees of tension with the 
same degree of filling; or conversely, various degrees of filling may coincide with the same tension. The muscle of its walls has the power of altering its length to accommodate the contents without changing its tension, just as we can voluntarily adjust the grasp of the hand so as to exercise the same pressure on a large or on a small ball. There is also reason to believe that the muscular coat of the small blood vessels, which prevents their over-distension by the pressure of the blood, has properties of the same kind.

Something of the same kind is shown by the voluntary muscle of the vertebrate; but in this case it is more directly brought about through the nervous system. After removal of certain higher parts of the brain, it is found that a limb offers resistance to a change in position, because some of its muscles are in a state of shortening. When this resistance is overcome, the limb remains in the position in which it has been placed, although its own weight may have to be held up against gravity. This reaction is due to the stimulation of certain nerves in the muscle substance, which convey messages to the nerve centres, and the result is a reflex stimulation of nerve fibres causing the peculiar form of contraction. There is evidence that this "postural" state requires the expenditure of much less energy than the voluntary production of the same degree of shortening. It appears that conditions of this kind are to be met with in some forms of "contracture," met with after injury, although not directly due to it (P., pp. 333, etc.).

The suggestion has been made that it is the sarcoplasm of the muscle that is responsible for the phenomena spoken of in the preceding paragraph. But the proof is not complete.

\section{Energy for Other Purposes}

From various statements in the previous pages of this book, it will be realised that a supply of energy is needed for such purposes as raising osmotic pressure, chemical reactions in which potential is raised, and so forth.

A useful index of the amount of energy required by an organ is the oxygen consumed by it, since oxidation is the source of the energy. This can be found by comparing the oxygen present in the blood going to the organ with that in the blood leaving it in a given time. This has been done in the cases of the secreting glands and the voluntary muscles already mentioned.

All living cells are found to consume oxygen, although it is not always obvious for what purpose they require energy. It has been suggested that it is to prevent diffusion, to maintain the integrity of membranes, and other purposes of this kind. 


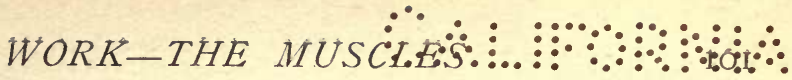

\section{The Maintenance and Regulation of Temperature}

In the first stage of muscular contraction, in which the potential energy is converted into tension, no loss in the form of heat is to be detected. That is, the whole of the potential energy lost appears in the form of mechanical tension, which can perform external work. If no external work is done, on the other hand, this energy becomes heat, and there is always heat produced in the restitution phase, since only a part of the energy obtained by combustion is stored as potential energy in the muscle system. We see, therefore, how the temperature of warm-blooded animals is kept up by muscular activity. The advantage of having a raised temperature is that the numerous processes, physical and chemical, go on at a faster rate, the former being less affected than the latter. It is even a debateable question whether the raised temperature in fever is not beneficial in the destruction and elimination of the bacteria and the poisons they produce.

The heat produced in muscular activity serves, then, to maintain the raised temperature in warm-blooded animals. But in muscular exercise too much is produced, and we become too hot. How do we get rid of the excess? The most effective way is the familiar one of sweating, since the evaporation of water requires a large amount of heat energy, which is drawn from the skin and indirectly from the blood. Evaporation of water from the lungs must also be added. A less effective way is by widening the blood vessels in the skin and allowing more loss by radiation, and by heating the air by conduction.

In hot weather we make use of yet another means, that is, by reducing muscular activity as far as possible.

In cold weather we diminish loss by narrowing the blood vessels of the skin, and we increase production by greater muscular activity. One form of the latter is "shivering"-an automatic method of keeping warm. A hibernating mammal, on waking up, raises his temperature in this way with rapidity.

Thus, the most effective way of lowering the temperature is by sweating ; of raising it is by muscular activity.

Since the source of our energy is food-and we need less heat energy in hot weather because we lose less to the surroundings - it is clear that less food is required in the summer.

There are, as we see, several factors involved in the regulation of our temperature, so that the necessity of a co-ordinating centre is obvious. Such a centre has been found in a part of the brain, situated between the highest intellectual parts and the more automatic parts. This centre is so arranged as to be sensitive to the temperature of the blood passing through it. If this tempera- 
ture is raised, the various means for increasing loss of heat and decreasing its production are set to work. If the temperature is lowered, those for decreasing loss and for increasing production are set to work.

The mechanism for controlling loss of heat appears to have been developed later in the course of evolution than that regulating production. It seems natural that an animal, finding itself getting too hot from exercise, should diminish first of all the amount of heat being produced, if circumstances permit becoming quiet. 


\section{STIMULATION-THE SENSES}

IN order that any organism may be able to make use of, or adapt itself to occurrences, in the outer world, it must possess means of obtaining knowledge of what is going on there. The various things that happen must, in some way, produce changes in the outer surface of the organism that is accessible to their influence. In other words, there must be structures capable of being "stimulated," or changes produced in them, by the forms of energy that strike upon them.

When this has taken place, the nerves connected with these "receptors" or organs of sense, as we may now call them, convey messages to the brain. They are then perceived in consciousness in a manner at present inexplicable, and may, sooner or later, result in muscular activity adapted to take advantage of the information received.

It will be seen that we cannot properly separate the discussion of the senses from that of the nervous system, and we might have taken the latter into consideration first. But whichever order is chosen, it is impossible to treat either one without assuming or forestalling what must necessarily be described later. Indeed, although for convenience it is usual to subdivide physiological phenomena into sections, they are, in reality, all parts of one system acting as a whole. This will have been manifest to the reader already, and for this reason no physiological text-book can be understood by reading it through once.

If we take a frog whose central nervous system consists of the spinal cord only, a "spinal frog" as it is called, we shall find that by stimulating the skin in a variety of ways we can produce movements (E., p. 209). These are called "reflex," because the message conveyed to the nerve centre is "reflected back" along another set of nerves, and causes muscular contractions. The name "reflex" is thus given to those movements which result from a stimulus without necessarily involving conscious perception of the stimulus. The phenomena of consciousness are only present when the highest part of the brain, the cerebral hemispheres, are intact. 
There must be channels along which the messages are conveyed to the nerve centres and back again to the muscles. These are the white threads called "nerves" (E., p. 209), which consist of a number of separate fibres, each carrying its own message apart from the rest. Nothing can be seen to happen either in the nerve or in the nerve centres. In the nerve-muscle preparation which we made previously, a stimulus applied at the far end of the nerve caused the muscle to contract, although there was no sign of anything passing along the nerve.

The student should examine the general arrangement of the central nervous system in a frog or rat (E., p. 209). But, at the present stage, details are unnecessary.

Let us next see what are the different kinds of sensations we receive from various external agencies. If the skin is pinched, we feel pain. If touched gently, there is no pain, but a sensation of a different kind. If a warm object is held near the skin, we have a sensation of heat. A cold object produces a sensation which is distinct from that of heat. All these are from the skin. By the eyes we perceive light. By the ears, sound. By another receptor, anatomically associated with that for the perception of sound, but having no physiological connection with it, we are informed of changes in our position in space, or our relationship to the direction of gravity. By the nose we smell, and by the tongue we taste. There are, thus, nine different kinds of sensation, each corresponding to some distinct property of external nature. The receptors which enable these sensations to take place must therefore each possess a structure which is appropriate to some particular form of external energy, so that a change may be effected in it by that form of energy when it obtains access to the receptor. A structure sensitive to light would be unaffected by sound waves, and so on. A not inappropriate illustration, as we shall see later, would be a photographic plate, in which chemical changes are produced by light, but not by sound. The change brought about in the receptor must be of such a nature and magnitude as to act as a stimulus to the ends of the nerves which arise from this receptor. We saw that pressure is able to stimulate a nerve when applied directly, but, in order to do so, it must be far greater than the degree of pressure involved in the sense of touch. It would seem, in this case of touch, that all that is necessary is some form of mechanical magnification of the action of the external agent. In other cases, as those of sound and light, the nerve itself is unaffected by them (E., p. 2IO), and it is necessary that they shall set into activity some mechanism which has the result of producing a form of stimulus to which the nerve is sensible. It appears that the energy value of an actual stimulus to which a sense organ can 
respond is not great enough to stimulate the nerve endings, even when converted into an appropriate form. It must act, therefore, as a trigger, or an electrical relay, setting off some store of potential energy present in the receptor mechanism.

All evidence available goes to show that, so long as a nerve fibre is stimulated at all, the process set up in it, and passing as a disturbance along it, is the same in all kinds of nerves, and always of the same magnitude. We have seen this to be the case with muscle, and it has also been shown, experimentally, to be the same with motor, efferent nerves. But the direct experimental proof is yet wanting for sensory, afferent nerves. The way in which the fact applies to the phenomena of sensation is expressed in the law known as that of "specific sense energies," a somewhat unintelligible phrase. What is meant is that, whatever the manner in which a nerve connecting a special receptor with the brain is stimulated, the sensation is always that associated with stimulation of this organ by its appropriate form of external energy. It matters not how the nerve from the eye is stimulated; the sensation is that of light. The clearest case is that of one of the nerves of taste, which passes through the ear in a way accessible to stimuli. Whether these stimuli be electrical, mechanical, thermal, or chemical, the sensation is one of taste, and nothing else. The object of each receptor mechanism is then to provide a stimulus of some sort to its nerve, no matter what. All that is necessary is that the arrangement shall be such that the external influence shall effect a change which actuates a stimulating agency.

The process may be illustrated thus: the nerve may be compared to an electrical circuit which can be connected up to a battery by closing a switch. It does not matter how this switch is closed. But, if light be the agent, it is clear that something sensitive to light must be present and be made to close the switch, say, by a current produced in an electro-magnet by a photo-chemical cell. If by sound, something similar to a microphone, and so on. These examples are not to be understood as implying that such are the actual means adopted in the eye and the ear.

In physical measurements we can convert any form of energy into an electrical current by a proper means, and in the physiology of the senses any form of outside stimulus is converted into one and the same form of nerve impulse.

But, it will naturally be asked, how can we distinguish sights from sounds, taste from touch, if the messages differ only as regards the particular nerve by which they arrive at the brain? We here come into contact with the mysterious relation between consciousness and the physiological changes in the brain. All that can be said is that when a particular region of the brain is set into 
activity, we experience something which differs in quality from that associated with the activity of another region. And this applies down to the individual cell at the end of each nerve fibre.

It is somewhat as if a man lives in an office in which electric bells are fitted in various positions on the walls. The bells are al alike, but each is connected with a different kind of factory in th town. When the bell in one corner rings, the man knows that a silk factory is at work; when that in another corner rings, a bras: foundry starts work, and so on. But we have also to suppose tha the visualising power of the man is good enough to picture the factories as if he were there.

When a message comes along a nerve fibre from the foot or th hand, we refer it correctly to its place of origin, although there i: no reason to suppose that the process in the nerve fibre itsel differs in the two cases. It is merely that it passes to a differen place in the brain. The psychological reader will recognise tha we are concerned with what has been called "local sign."

We may now proceed to discuss, more or less briefly, the different kinds of receptors.

Physiologically, the most primitive and simple is the sensation of pain, associated with the action of something that is likely to cause actual injury. In this case there are no specialised receptors The nerve fibres come to an end between ordinary cells, and the stimulus acts directly upon the nerve itself. The sensitivenes is therefore not great. It would indeed be a disadvantage if $i$ were, since the muscular reactions due to pain are usually powerful and it would be undesirable to provoke them unless there wer actual risk of injury. The protective function of pain would be defeated if innocuous contacts excited it. The skin contains nerve endings of this kind, along with specialised receptors. The cel layer covering the front of the eye contains no other kind of sense organ, and is sensitive to pain only.

The sense of touch is associated with special receptor: adjusted to be responsive to very slight degrees of deformation These receptors are localised in spots in the skin, usually arounc hairs. Although the presence of the hairs increases the sensibility apparently by some kind of lever action, the sensation of touch is still present when the hairs are removed. The whiskers of the cal are extremely sensitive organs of touch, and their roots in the skir are copiously supplied with nerves. The structure of the various receptor organs for touch does not throw much light on the way in which they act.

Heat and Cold.-If an object, applied to the skin, is at a higher temperature than the skin itself, we call it warm; if at a lowe temperature, it is said to be cold. Like touch, there are separate 
spots sensitive to temperature, and, a rather curious thing, there are different receptors for heat and cold (E., p. 2 I I). The nature of a specialised receptor may, to some extent, be realised by stimulating with an electrical current a spot sensitive to cold, for example. A sensation of cold, and no other, is produced, but the strength of the stimulus necessary is very much greater than when it is the normal one of cold. This means that the mechanism is specially adjusted to be affected by the withdrawal of heat. In what way this is done we cannot say. It has been suggested that it may be by some chemical reaction which is very sensitive to change of temperature, or some effect on volume may be concerned.

Taste and Smell.-These may be called "chemical senses," because they depend on the properties of substances acting in watery solution on the receptors. But it must be remembered that the properties are not ordinary chemical ones, since there are a number of compounds which taste sweet, although there is nothing in common in their chemical nature.

The skin of fishes has a kind of generalised chemical sense, such as would naturally be expected to make its appearance at an early stage in evolution, in response to the variety of chemical substances given off to water by other animals and plants. It seems probable that the senses of smell and of taste of the higher animals have developed from this. It should be remembered also that the sense of smell plays a large part in the life of water animals.

In one case, that of acid substances, the taste is definitely in relation to the hydrogen-ion concentration.

With the exception of smell, the senses hitherto described require the actual contact of objects with the surface of the body, and they give us no warning of the approach of distant influences. Although touch gives valuable information of the properties of objects, and guides us in muscular movements, while smell, especially in certain organisms, is of value in warning of distant occurrences, it is by sight and hearing that accurate information is obtained of such things. It is to these " distance receptors" that we owe the greater part of our higher intellectual life. The mode of action of the receptors in these cases is a complex one, but, nevertheless, it may be said that we know more about it, up to a certain point, than about the apparently simpler cases.

Hearing or the Receptor for Sound.-The phenomenon in the outer world that arouses in us the sensation of sound is an alternate condensation and rarefaction of the material of which bodies are composed, transmitted in the form of waves. If we confine our attention to one point in the air, for example, we notice that the air becomes alternately denser and rarer. What is known as the pitch of a note is the number of times per second that this process takes 
place. The number of vibrations in what we call musical sounds lies between about 40,000 and 30 per second. What we call the loudness of a sound depends on the degree of changes in density, or, what comes to the same thing, to the amplitude of the backward and forward movement of the particles of the vibrating substance, since the more they have congregated together at one moment, the further have they come. There is another property of sound, shown most markedly by the difference between the same note played on the violin and on the flute. This is called quality, and will be referred to presently.

What the ear has to do, then, is to transform periodic changes in density of the air into something of the nature of an actual pressure or pull upon the endings of nerves, in such a way as to stimulate them. When these air vibrations enter the ear, they come against the "drum," a membrane stretched across the passage. The membrane is caused to move in and out by the periodic changes of pressure upon it. The important point is that it moves equally well to any rate of vibration, on account of the fact that it has no particular rate of its own, as an ordinary drum has. This is partly due to the shape of the membrane and partly to the fact that it is connected to a series of small bones which prevent its free vibration. The result is that it follows exactly the smallest changes in air pressure and passes the movement on to the end of the chain of bones, unaltered in wave form, but, owing to the lever action of the bones, diminished in amplitude and correspondingly increased in force. The further end of the bony lever is fixed to a small membrane covering an aperture at the end of a canal in hard bone. This canal has a spiral form, like a snail's shell, hence called "cochlea," from the Latin name. It contains liquid, and on this liquid a periodic series of pressures is exerted by the end of the chain of bones. Suspended in the liquid is a complex structure in which the auditory nerve ends, the "organ of Corti." The details of this organ can only be given here in a general way, so far as necessary to understand its mode of action.

But, first of all, what is the nature of the vibrations set up in the liquid by the periodic changes of pressure upon it? Let us see what would happen supposing that the sound waves in the air hit directly the end of such a column of liquid. We know that they are transmitted, and may be transferred to air again at the opposite end of the column. Sound can only be transmitted owing to the elasticity and the compressibility of the material conducting it. If this material were devoid of elasticity, the particles in vibration would not return after being displaced, and if it were incompressible, the alternate states of condensation and rarefaction would be impossible. Although liquids are almost perfectly elastic 
in the physical sense, they are, compared with gases, only very slightly compressible. The result is that the amplitude of the sound vibrations in a liquid is excessively minute, but the force involved is of correspondingly increased magnitude. We have seen that the changes of pressure exercised by the chain of bones

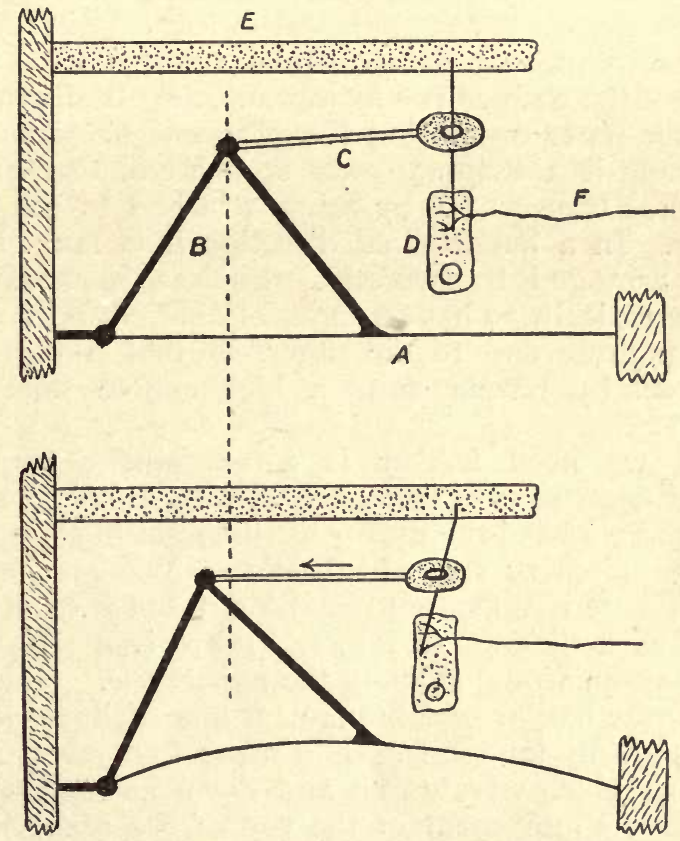

Fig. 4.-Mechanism of the Organ of Corti.

Upper diagram -at rest. Lower diagram - when displaced by vibration.

A, represents the basilar membrane.

$B$, the arch of Corti.

$\mathrm{C}$, the reticular membrane.

$\mathrm{D}$, one of the hair cells.

$\mathrm{E}$, the tectorial membrane.

$\mathrm{F}$, a fibre of the auditory nerve.

on the liquid in the cochlea correspond exactly with those in the air, in contact with the drum, except that their amplitude is decreased and their force increased. This would clearly be an advantage in transmitting them to liquid. But, in other respects, they must be precisely similar in the liquid to what would have been the case if the sound waves had impinged directly on the liquid itself. In this liquid we have, then, waves identical with those of sound. 
Next, supposing that we have immersed in water a spring which is capable of vibration at a certain rate, like the wires of a piano, and that we send, by some means, sound waves of this rate into the water. The spring will be set into sympathetic vibration by resonance in the way previously explained (p. 52 above). But if its rate is not that of the sound waves, it will remain at rest. Have we then anything that might act in this way in the cochlea?

One of the component parts of the receptor structure immersed in the liquid of the cochlea is a membrane, the "basilar membrane," to which other parts composing the organ of Corti are attached. This membrane is a strip narrower at one end than at the other, and is stretched transversely by being attached to the bony walls at both sides. In a longitudinal direction it is lax. It also contains fibres arranged transversely. Such a membrane can be shown mathematically to have a series of different rates of vibration in order from one end to the other, so that a narrow section would respond by resonance to a higher note than a broader one (E., p. 212).

All that we need further is a mechanism by which the vibrations of each section can be made to stimulate a particular nerve fibre and we have the means of distinguishing between notes of a different pitch or rate of vibration. The precise means by which this is done is difficult to make out, but it seems to be that represented as a diagram in Fig. 4. There is a series of arches, jointed at the top, arranged along the membrane. One foot of the arch rests on the basilar membrane near one of its attachments, so that it is practically immobile. The other foot rests on a part of the membrane which vibrates up and down as represented. The result of this is a movement of the top of the arch chiefly in the direction from right to left in the plane of the paper, and back again in the opposite direction. Attached to these arches is a membrane ("reticular") with holes in it. Through these holes project stiff hairs attached to cells below it. The points of the hairs appear to be more or less fixed by being stuck against another soft membrane ("tectorial"). When the reticular membrane therefore is pulled backwards and forwards by the up and down movements of the basilar membrane, the base of the hair is pulled through, or together with, the cell to which it is attached, and exerts pressure on the termination of the auditory nerve which ramifies in or upon the cell.

It will be clear that there must be as many nerve fibres and elements of the organ of Corti as it is possible to distinguish in difference of pitch. It is said that about I I,000 different notes can be distinguished, and the number of fibres in the cochlear division of the auditory nerve has been found to be 14,000 .

The number 
of Corti elements appears to be sufficient also, but they are more difficult to estimate.

So long as a disturbance is sent along a particular nerve fibre, it does not matter whether or not this disturbance corresponds in its form, or in the rate at which separate impulses follow one another, with the sound vibrations in the air. When it reaches a particular region of the brain, we have the sensation of a certain note, in which the separate vibrations are not distinguished.

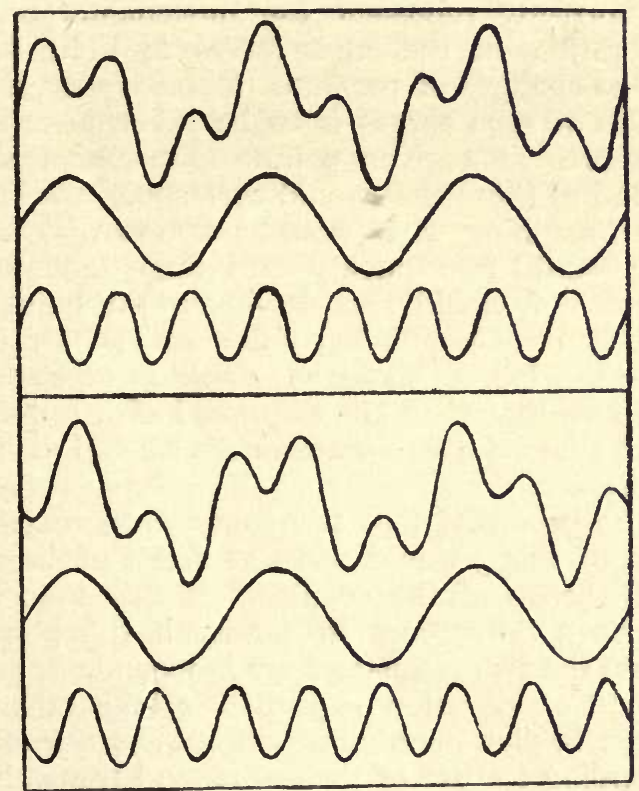

FIG. 5. - Compound Wave Forms resulting from Fusion of a Vibration of a certain rate with one of twice that rate, in two different Phase Relations with each other.

It should be mentioned that the view according to which the basilar membrane responds to different rates of vibration by resonance is due, in the main, to Helmholtz. Although it is more in agreement with all the facts than other theories, there are some which assert that the basilar membrane vibrates, as a whole, to all notes, the wave form of which is held to be transmitted to the brain in all its detail, so that the analysis is performed there.

A few words are necessary on the perception of quality in musical sounds. Why is the same note played on the violin and on the flute so different? It is because, in the first case, the 
fundamental note itself is accompanied by a large number of other rates of vibration due to the subdivision of the string into various numbers of parts of shorter length, each giving rise to a note of higher pitch, in some multiple of the fundamental. The presence of these harmonics, as they are sometimes called, can be detected by the use of appropriate resonators. The form of the air waves resulting from the combination of these harmonics with the fundamental note is usually represented by compound sine curves, such as those of Fig. 5. But it is to be remembered that although such curves correctly represent the movements of particles in transverse vibration, or the ether waves of light, they are only diagrams of the changes of pressure in sound wayes. The height of the ordinates of such curves is to be taken as representing the series of pressures at a given point. One may/realise, to some extent, the kind of thing that would correspond to the upper curve of Fig. 5, by imagining what would be shown by a manometer. The pressure would rise quickly and fall gradually. Whereas in the lower curve it would rise slowly and fall quickly. In some other cases it would, after having fallen somewhat, rise again and then continue the fall, and so on. Looking at such curves it is difficult to believe that they are composed of a number of simple vibrations, and that an appropriate resonator can pick out any one from amongst them.

It might be supposed that the comparison of two such wave forms as those of Fig. 5 would serve as a test of the correctness of the resonator theory of the cochlea. If this theory be correct, there should be no difference in quality if the phase relation is altered, because the two resonators are independent, and each picks up its own rate of vibration regardless of the other. If, on the other hand, the basilar membrane vibrates, as a whole, in a wave form corresponding to that of the sound, and transmits this by the nerves to the brain to be analysed there, then phase difference should be appreciated. Unfortunately, there is no agreement on the fact. But it seems rather doubtful whether the methods used by those who state that phase difference is of importance were such as to exclude other effects on the components of the complex waves.

\section{The Eye-Receptor for Light}

The eye may be said to be the most accurately adjusted of all our receptor organs. It is adapted by its movements and great sensibility to give us more correct and valuable information about things that are happening, both near and at a distance, than any other organ of sense.

As previously mentioned, light consists 'of transverse waves in 
a medium which is not material, at least, not matter in the ordinary form.

Some idea of the kind of vibration in question may be gathered by watching a sea-gull floating on the sea. It will be seen to rise and fall, as a wave passes under it, without permanently altering its position in relation to objects around it. Such a vibration is in one direction only, the vertical, and in the case of light would be called a polarised beam. Ordinary light consists of vibrations in all directions at the various angles with this.

We have already seen that large quantities of energy are transmitted to us from the sun by wave motion of this kind, and that it is only a limited range of wave lengths or rates of vibration that we perceive as light, although the longer wave lengths can be perceived by the heat receptors of the skin and the shorter ones have powerful chemical effects. Waves of a greater length than the longest of the solar spectrum can be produced by electric discharges, and form the basis of wireless telegraphy. Waves much shorter than the ultra-violet of the spectrum are known as X-rays or Röntgen-rays, which have remarkable powers of penetrating substances opaque to ordinary light.

The manner in which the vibrations of wireless telegraphy, electric waves, are produced, reminds us that light is an electrical disturbance, although there are still difficulties to be explained in connection with the relationship between the moving electrons and the transmission in wave form.

The first question that arises in connection with the perception of light is, what effects capable of being used to stimulate nerve fibres does light produce when it falls upon material objects? Although, as would be expected, there are certain electrical effects to be detected, the most obvious ones are heat and chemical change. The means of perceiving the former are not nearly delicate enough, and there is every reason to believe that the immediate cause of the stimulation of the endings of the optic nerve is by a so-called photo-chemical reaction. The photographic plate shows us how sensitive such a reaction can be made, although the mechanism in the eye is much more sensitive than the most rapid plate. The fact of a chemical change produced by light is readily seen in the case of "printing out paper." The change does not go so far on the dry plate used in the camera as to be visible, but the fact that an image appears on development by a reducing agent shows that a chemical effect had been brought about.

The skin of some lower organisms appears to be sensitive to light, but such a general sensibility would only give information of the approach of another object by the shadow cast by it, and it is not until specialised eyes are developed that the perception of 
light takes the important place that is attained by it. It is, however, very early in the scale of evolution that eyes are found. The jelly fish possess them, although they do not possess the necessary elaboration of structure required to form distinct images. The perception of sound, contrary to that of light, seems to be a comparatively recent development.

The layer or coat at the back of the eye in which the nerve fibres end is called the "retina" (E., p. 2I4). It contains a substance called "visual purple," which is sensitive to light (E., p. 212). We saw, in discussing the action of chlorophyll, that in order that light energy should have any effect it must be absorbed, and the magnitude of the effect is naturally in proportion to the amount of light absorbed. Investigations of the properties of visual purple have shown that its absorptive power for different parts of the spectrum agrees with the sensibility of the retina to these parts. Further, the effect of light in bleaching the pigment follows the same course, and also does the apparent brightness of the different parts of the spectrum. We are therefore justified in regarding this pigment as the seat of the photo-chemical reaction at the basis of vision (see P., p. 521 ).

But a mere sensibility to light would be of comparatively little value. It is necessary to have a means of producing a picture of external objects on the retina, so that different parts of this picture may stimulate separate nerve fibres, and a representation of it be conveyed to the brain.

A familiar method of producing a picture on a sensitive surface is that of the photographic camera, and it will be instructive to compare its essential parts with the corresponding parts of the eye. The student should examine these parts in the eye of an ox (E., p. 2I3). The sensitive plate, as we saw, is represented by the retina, and on this an image is formed by means of a lens which gives a real image. Such a lens consists of the convex surface of a medium having a higher refractive index than air. The ray from each point of an external object is bent towards the centre of the lens when it strikes it, and in proportion to the distance from the centre of the lens at which the ray enters. At a certain distance behind the lens an image is produced. If the object is distant, the focal plane, as it is called, is nearer to the lens than if the object is at a less distance, and the size of the image is less, the nearer it is to the lens (E., p. I63). That part of the eye which takes the place of the lens of the camera is not, as might be thought, that structure which is actually called the "lens" of the eye. This plays a comparatively small part in the formation of an image, but has another function, as will be seen presently. The actual lens is the front clear spherical surface of the eye, known as 
the cornea. This can easily be seen by a simple experiment on the eye of an albino rabbit (E., p. 213). But remembrance of the fact that the liquid in the eye has a higher refractive index than air, and that it is bounded by a spherical surface, is sufficient to bring conviction. The material of the lens itself has a refractive index not much higher than that of the liquid in which it lies, so that the actual refraction due to it is not great. What it does is to adjust the focal length of the dioptric system of the eye, so that sharp images of objects at various distances from the eye may be formed on the retina. This it does by altering its curvature. The greater the curvature of a refracting surface, the shorter its focus. Many photographic lenses are double, so that each part can be used separately. One part is often of shorter focus than the other, and can easily be seen to have a more curved surface. The mechanism by which accommodation to objects at different distances is effected in the case of the eye of the higher vertebrates is, briefly, as follows :The lens is an elastic body, which has, when released from its position in the eye, a particular natural curvature. In its normal position in the eye it is pulled flatter by the way in which it is held stretched between membranes in front of it and behind it, which are kept in a state of tension. There is, further, a ring of muscle, the ciliary muscle, whose fibres are arranged in such a way that when they contract they pull the place to which the suspension of the lens is attached nearer to the lens itself, and thus lessen the tension on it, allowing it to approximate more or less to its natural curvature. Its focal length is diminished, and the image of a near object, which would otherwise be formed beyond the retina, is thus brought to lie nearer to the lens and on the retina itself.

This is not, of course, the way in which the photographer adjusts the focus of his camera; he moves the lens backwards and forwards, since its curvature is fixed. In some of the lower animals, indeed, a means of accommodation like that of the camera is adopted, a muscle being present to change the distance of the lens from the retina.

There are two further arrangements common to the camera and to the eye. The diaphragm, which enables sharper images to be formed by limiting the part of the lens used to the middle, naturally with loss of light, is represented by the iris, the coloured screen with the aperture, the pupil, in front of the lens. The iris contains muscular fibres arranged in a radial direction, which enlarge the pupil when they contract, and others in a circular direction, which narrow it. In the eye, however, the chief use of the iris is to prevent excess of light from reaching the retina, and the improvement in sharpness of vision is secondary, although advantageous when the light is strong enough to permit it. 
The other arrangement corresponds to the bellows of the camera, to keep out stray light from acting on the plate. It is represented by the eye-ball itself, which is lined by a layer of cells containing black pigment. This pigment layer is to be found in the very simplest eyes, and is clearly of much importance.

The retina in the vertebrate is a very complex structure of several layers of different kinds of cells (E., p. 2I4). But several of these layers properly belong, not to the receptor organ itself, but to the nerve centres. In the cuttle-fish they are in a separate nervous mass, outside the eye. The actual receptive layer is that of the rods and cones. That the cones are the elements concerned with accurate vision is obvious from the facts that this is in direct relation to the number of cones present in a given area, and that the central part of the retina, where the most accurate vision is present, contains cones only. The function of the rods is somewhat obscure, but their nervous connections are very similar to those of the cones, and it seems that they must also be percipient elements of some kind. The rods and cones lie in a solution containing visual purple, and when a bright part of an image is formed at a point on the retina, the photo-chemical change in the sensitive substance causes the cones, and perhaps the rods, with which it is in contact to be affected in such a way as to stimulate the nerve fibres in connection with them. Whether this is by a chemical action or by the resonance of molecules to particular wave lengths is not yet clear, but the phenomena of after-images, to be referred to below, suggest that the former is the case. We saw, in discussing the chlorophyll system, that light energy is absorbed by a system for the reason that a certain molecular group has a vibration rate which is in unison with that of the light which it absorbs. The resonant vibrations may be great enough to result in chemical decomposition.

That a change is produced in the visual purple such that a certain time is necessary for a return to normal is familiar in the negative after-images, where a part of the retina, on which the image of a bright object has fallen, remains for a time less sensitive, thus causing the appearance of a dark patch in the field of view. The regeneration of the visual purple is of interest, because a similar phenomenon is met with in some of the simpler photochemical reactions, such as that of silver chloride. Suppose that we have some of this compound in a sealed tube and allow sunlight to act upon it. It turns purple, chlorine being given off, and metallic silver in a finely divided, colloidal form being left. Now chlorine and silver have a strong affinity for one another, and if the tube be placed in the dark they recombine. But this recombination takes place whether light is acting or not, so 
that it must always be going on, even while light is acting. Hence there is a balance between the decomposing action of light and the recombination of the products, such that the composition of the system depends on the intensity of the illumination. Since this balance only lasts as long as external energy of light is being supplied, it is not a true chemical equilibrium. The reason why the image formed on a photographic plate does not disappear after exposure is because there is gelatin present, and the chlorine or bromine liberated combines with the gelatin, and is not available to recombine with the silver.

Other phenomena, whose meaning is not yet clear, are produced in the retina by light (P., pp. 519-525). Among these there is an interesting electrical change.

The perception of colour is a question about which opinions are somewhat at variance. When we look at the spectrum there are to most of us six distinct colours in it-red, orange, yellow, green, blue, and violet. A few people, like Newton, see a distinct colour, indigo, between blue and violet. All other colours can be formed by combinations of these with each other and with black or white. Whether there is a distinct variety of visual purple for each of the six primary colours, or whether each of these affects the same substance in a different way, is unknown. It is certain, however, that visual purple, as we know it, absorbs light of all parts of the spectrum; but this may be due to its being a mixture of six substances.

\section{Position-receptors}

These receptors are of two kinds, and they give us information of our position in relation to the direction of gravity or of the direction in which our bodies have moved. They may be called position-receptors, and make use either of the weight of particles to stimulate nerve endings, or of the inertia of liquid in its refusal to take up suddenly the movement of a vessel which contains it.

Practically all multicellular animals, and plants too, have organs by which the direction of gravity is made known to them, and reactions set up to bring them into a definite position in relation to it. We find sacs full of liquid containing one or more solid particles. Projecting into the liquid are hairs attached to cells in connection with nerve fibres. Such organs are known as statocysts in animals (E., p. 214). In plants, starch grains in cells appear to perform similar functions. When the organ or cell is in the normal position in relation to the vertical, the grains lie on the lowest part of the sacs and stimulate the nerves or protoplasm in that region. If the position changes, the grains stimulate hairs in a different place, and the change of position is known and corrected. This mode of 
action was made clear by an ingenious experiment with the crayfish, which sheds the inner lining of its statocysts along with its shell. The cavity of the statocysts is in communication with the outside by a small pore; grains of sand are normally taken in to replace those lost when the lining is shed. If nothing but iron filings is available, these are taken in and can then be caused to press upon various parts of the wall of the statocyst by bringing a magnet near the animal, which then proceeds to turn over in the way it would have done if this part of the statocyst had been brought in the normal way to be the lowest part of the sac. In the vertebrate, these functions appear to be undertaken by those parts of the internal ear known as utricle and saccule, which have structures similar to those of the statocysts of lower animals, although some observers hold that the former have auditory functions. It must be remembered that the touch and pressure receptors in the skin and the muscles serve to give us information of that part upon which pressure is being exerted, or of what part is being stretched, and thus indirectly of the relation of our bodies to the vertical direction.

The second kind of position-receptor is that interesting organ known as the labyrinth or semi-circular canals, present only in vertebrates. There are three of these on each side of the head, forming a part of the internal ear, although they have nothing to do with the perception of sound. Each is in the form of a hollow ring, and the three are connected together at one part, so that there is communication between their internal space. The plane of each ring is at right angles to that of the two others, so that the three canals are situated in the three dimensions of space, corresponding to the length, breadth, and thickness of an object (E., p. 214).

Suppose now that the whole arrangement is moved quickly in a plane which coincides with that of one of the canals. The liquid contained in it will not partake, to any great extent, in the movement of the walls, because the friction between the layers of the liquid is not sufficiently great to convey the motion to the whole mass at once. In other words, the walls are moved along, leaving the liquid behind. Those canals which are at right angles to the plane of movement will, naturally, not be affected in such a way as to cause relative displacement of the liquid and the walls. But if the direction of movement is such as to have components affecting more than one canal, the effect on each will be inversely proportional to the angle which its plane makes with that of the movement.

How is this effect made to stimulate.nerve-endings? At one end of each canal there is a dilated portion, and on one part of its wall there is a protruding mass of cells with long hairs reaching 
into the liquid. These hair-cells are connected with nerve fibres and, when the apparatus moves, the hairs are dragged through the water, since this does not move with them. The result is that they are deflected, and their bases press upon the cells and nerves. The process may be compared to "catching a crab" in rowing, the hairs corresponding to oars, the nerve cells to the oarsman, and the walls of the canal to the boat.

It will be seen that the function of this apparatus is chiefly for the perception of more rapid movements in space than could be detected by the statocyst. If the movement is slow there will not be much relative displacement of the liquid and the hairs, and when at rest in any position there will be no stimulus at all. The statocysts, on the other hand, are arranged to indicate permanent changes of position, and are not very sensitive to rapid movements.

As mentioned above, we do not depend entirely on the labyrinth for information as to position. In addition to differences of pressure on that part of the skin in contact with solid objects, there are receptor organs in the muscles themselves, which indicate changes of tension in them, according to their position and that of the parts to which they are attached. Moreover, the joints are provided with nerves. Together, these form the receptor organs for the so-called muscular sense.

It will be noticed that we have here a distinct class of receptors, affording information of the state of parts in our own bodies, as distinguished from that of external objects. The former class is known as that of the intero-ceptors; the latter are the extero-ceptors. Of the intero-ceptors, the most important are the proprio-ceptors of the muscles, which afford information of the state of activity of these organs. The centres receive messages, as it were, as to whether the command has been obeyed, clearly of great importance in the carrying out of complex movements, which depend on a series of acts.

The fact that certain sense-organs, especially the eye and the hand, are provided with muscles capable of moving them in any direction, is of much importance in the perception of direction in space, and of the forms and distances of external objects. It is by the co-ordination of these two organs that we learn, by experience, how to interpret the information given by either. The size of the image of an object on the retina would not inform us of the actual size of the object unless we had, at some previous time, moved the hand over it or some object of the same apparent size, and found the muscular effort necessary. 


\section{CHAPTER V}

\section{ADJUSTMENT-THE NERVOUS SYSTEM}

IN its simplest aspect, the central nervous system may be said to be concerned with the adjustment of the organism to external changes. We have seen how these outer changes are enabled, by appropriate receptors, to impress themselves and how the appropriate responses are made by muscular movements. We have now to try to understand something of the way in which the connection between them is made. In the physiological discussion of the functions of the nervous system, we are not concerned with the fact that the activity of the highest parts of the brain is associated with what we call the mind, with conscious knowledge of their activity. By the "highest" parts of the brain we mean those parts which are the most removed in anatomical relationship to incoming stimuli from that which we know to be the seat of the simplest reflex movements and to be devoid of consciousness, namely, the spinal cord (E., p. 2I5). It may be remarked here that the parts in question, the cerebral hemispheres, are developed in relation more especially to what we have recognised as the distancereceptors, the eye and the ear; and it may be noted that these are the receptors, together with the hand, chiefly concerned with the development of speech and the use of written language, without which intellectual growth would have been impossible. It need scarcely be said that in animals of high mental development, a large number of processes and much lapse of time may intervene between the reception of a message and the execution of the response appropriate to it.

When an impulse arrives in the nerve centre along a fibre from a receptor organ, what happens to it? We find, by histological examination (E., p. 2I6), that the fibre divides, and that its branches are connected to a cell containing a nucleus, usually to fibres proceeding from the cell. - In the simplest conceivable case, this cell is the "motor centre" of some particular muscle. That is, the nerve fibre given off by it passes to a muscle, and when set into action causes contraction of that muscle. This is the most elementary form of "reflex action," and is rarely met with. It may be represented by the parts $\mathrm{B}$ and $\mathrm{D}$ of the diagram in Fig. 6 . 
A reflex is then the physiological unit of the central nervous system, but not the anatomical one. We see that at least two nerve cells are concerned, in addition to the receptor and the muscle, which latter may be called the "effector." These structural units of the nerve centres are known as "neurones," because the use of the name "nerve-cell" was found to lead to confusion. A neurone is a peculiar type of cell in that part of it consists of a fibre, the "nerve fibre," which may be of great length, sometimes several

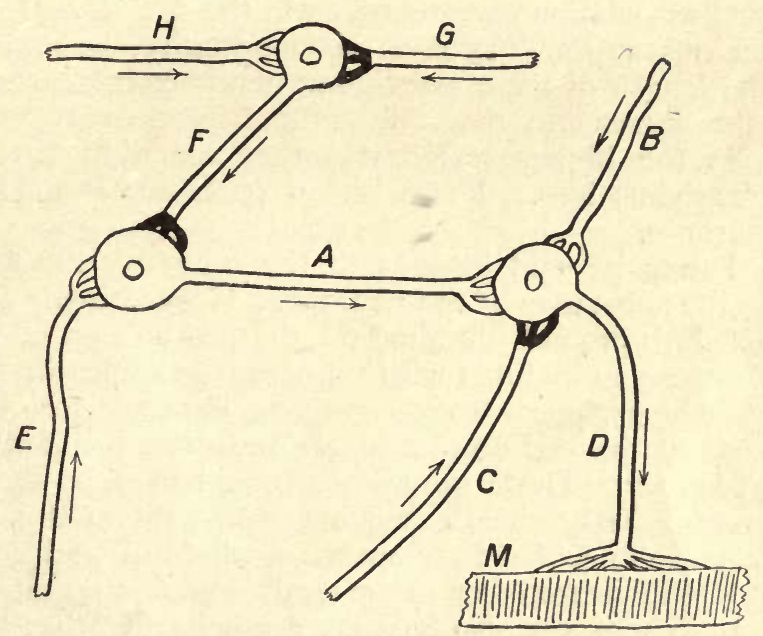

FIG. 6.-Diagram of the General Arrangements of the Central Nervous System.

A, excitatory association neurone.

$\mathrm{B}, \mathrm{E}$, and $\mathrm{H}$, excitatory afferent (sensory) nerve fibres.

$\mathrm{C}$ and $\mathrm{G}$, inhibitory afferent nerve fibres.

$\mathrm{D}$, motor (efferent) neurone, ending in a muscle.

$\mathrm{F}$, inhibitory association neurone.

The excitatory synapses are white, the inhibitory ones black.

feet; but it is, nevertheless, as truly a part of the cell as the protruded pseudopodium of an amœba is. Its structure degenerates, and it loses the power of conduction after it has been cut off from the part of the cell containing the nucleus. This fact enables us to obtain some information as to the nature of the connection between the two or more neurones forming a "reflex arc." If the sensory or "afferent" fibre taking a message to the centre be cut, the portion beyond the place of section degenerates, but only so far as the place where it joins another neurone. The process does not extend beyond the junction, which is called the "synapse." It is 
clear that there is not protoplasmic continuity, and that the two neurones are independent of one another, as far as their nutrition is concerned. The usual cell membrane intervenes, and is here called the "synaptic membrane," having some special properties. The physiological process must clearly be transmitted through this membrane or no reflex would result. This transmission is perhaps an electrical effect in which ions change places, but the process is still obscure in its details.

In the greater number of reflexes there are one or more intermediate or "association" neurones, as in the arc E, A, D of Fig. 6. As the nervous system increases in complexity, we find that the number and length of these association neurones increases, so that, while in the earthworm they only extend to one or two adjoining segments, in the higher vertebrates they reach to the cerebral hemispheres themselves. These higher parts are formed entirely of such neurones.

A word may be said here as to the nature of the protuplasm composing the substance of the neurone. If examined, while still alive, under dark ground illumination, the protoplasm of the body of the cell is seen to be filled with numerous granules in Brownian movement, which appear to congregate together for a time in various parts of the cell, so that larger and more brilliant particles are formed locally. These granules are said to show signs of the possession of a fatty sheath which stains with methylene blue. The movements show that they are suspended in liquid, so that the structure of the neurone is in general similar to that of other protoplasm. There are also reasons for believing that the nerve fibre process, "axon," as it is called, is also of a liquid nature.

It may be asked, what is the function of the nucleated part of the neurone? It may possibly act as a kind of relay, adding energy to a nerve impulse which has become weakened by passing through the synapse. But this is by no means certain. It has been shown that reflexes can take place after these parts of the neurones have been removed, as is possible in some animals. It is clear, however, that the continued life of the neurone depends on this nucleated part, as would be expected from the statements made in our first chapter with regard to the functions of the nucleus. If a part of the neurone is separated from the "trophic" influence of the nucleus, it dies, disintegrates, and ceases to carry impulses.

Comprehension of the general principles on which the central nervous system is constructed may be assisted by a short account of its evolution. In the lowest multicellular animals, the sponges, there are no structures comparable to nerves, although they possess effectors in the form of muscle cells under the layer of amœboid 
cells of the outer skin. The muscle cells are evidently stimulated to contraction by mechanical influences on the amoboid epithelial cells, which may in a sense be regarded as receptors, but of a very simple kind. In the sea-anemone there are more highly specialised cells in the outer epidermis, which have long, thin projections inwards, forming, with other similar fibres, a felt work between the outer layer and the muscular layer. These fibres ultimately end on muscle cells at a greater or less distance from the cell giving origin to them. Since they serve to elicit muscular movements at a distance from the point stimulated, they may with justice be called nerve fibres and, together with their epidermal cell bodies, form primitive receptor or afferent neurones. There is still no indication of nerve centres. The next stage is met with in the earthworm and elsewhere, and is the beginning of the synaptic system, which enables so much advance in adjustment and coordination to be made. We find that the nerve fibre does not proceed straight to a muscle cell, but it enters a nervous mass or "ganglion," and forms a synapse with processes of a neurone, whose cell body is found here. The axon of this neurone passes to a muscle cell, and is hence called a motor neurone. The advantage of such an arrangement is that the same muscle can be put into action from different sources, since more than one afferent neurone can form a synapse with it. Thus commences what is called the principle of the "final common path," where the neurones supplying a particular muscle serve as a common channel for the many reflexes in which this muscle takes part. In the earthworm there are also association neurones. Here the afferent fibre does not form at once a synapse with the motor neurone, but with another one which is entirely confined to the nerve centre. The axon of this neurone ends either directly on a motor neurone or only after the interposition of one or more further association neurones, which may end in a more distant part of the nervous system. As complexity and variety of adjustments increase, we find a more and more copious growth of these association neurones, extending to a greater and greater distance, so that the organism becomes a connected whole. Thus the general arrangement is that of a series of alternative loops or arcs (P., pp. 468 and 478), by which an impulse received, say in the foot, may either pass across as a spinal reflex in a neighbouring part of the spinal cord or by various other paths in the brain itself, including the cortex of the cerebral hemispheres.

There is a circumstance with respect to the receptor neurones in the vertebrate in which they differ from those of the invertebrate. It was mentioned above that cells in the epidermis of the latter organisms become specialised so as to act as more sensitive 
receptors. In this process their outer ends become elongated into protrusions of various kinds, so that the nucleated cell body recedes from the surface somewhat. But this does not proceed far until we arrive at the vertebrate, in which the cell bodies of the receptor neurones have receded nearly as far as the spinal cord itself, forming what are known as the dorsal root ganglia. In this way the ends of their axons are either merely situated between cells at the periphery, forming pain receptors, or are connected with the cells of specially developed receptors, such as were described in the preceding chapter.

Those association neurones which form the arcs extending through parts of the brain itself represent the complex co-ordinated activities in which thought and memory take part.

We must now return to consider some aspects of reflex action hitherto unmentioned. First of all, we should realise, by the examination of some of these, that they take place without the necessary participation of consciousness. The spinal frog (E., p. 2 I6) serves well for certain experiments. In ourselves, the quick withdrawal of the hand, when it touches a hot object, is done without the conscious intention of doing so, although the sensation and the fact of the reflex taking place are present in our consciousness. Although the afferent impulses from the skin receptors have travelled across by short arcs, branches from them have also proceeded to the brain by the long arcs.

The variety of reflexes in which the same muscle or group of muscles take part will probably be noticed in the experiments on the spinal frog. The importance of the final common path is shown here. Instead of having the receptors for each of these reflexes separately joined up to the muscle, they have merely to be connected to the motor centres of the muscles, directly or through intermediate neurones, and one set of out-going or efferent fibres suffices. But it is clear that the same final common path cannot be used for different reflexes at the same time, and if it is to be used quickly for a new reflex, the preceding one must be cut short. The discharge of a reflex arc lasts longer than the stimulus producing it, and it is frequently necessary to stop it more rapidly than it would cease if left to itself. This is done by a process of inhibition. We do not know what this actually consists in, beyond the fact that certain nerve fibres end on a muscle or nerve cell in such a way as to lessen or stop its activity, instead of increasing it, as the ordinary motor or excitatory fibres do. We have seen an example of it in the case of the intestinal muscle, and a very important one is that of the action of the vagus nerve on the heart (E, p. 2I7).

It must be understood that the process of inhibition is an actual 
effect on the discharging neurone, making it for the moment incapable of discharging. When the inhibitory influence ceases, the neurone has been put into a state of rest in preparation for taking its part in a new reflex act. Suppose that a motor neurone is discharging under constant stimulus from a receptor. Inhibition does not mean putting a block in the path of the stimuli, since the motor neurone would continue its discharge for some time afterwards. It is actually caused, by a direct influence, to stop discharging practically instantaneously. The muscle contracting under its discharge relaxes to its full length suddenly (P., pp. 4IO and 4I4).

Some of the ways in which inhibition works in nerve centres may be realised from Fig. 6 . The fibre c, when stimulated, inhibits the motor neurone directly. A reflex through an intermediate neurone can also be stopped by inhibiting this intermediate neurone, as by $F$. An interesting case is when a reflex is being elicited by stimulation of $\mathrm{E}$. If, at the same time, $\mathrm{H}$ is being stimulated, it sets the neurone $\mathrm{F}$ into activity, and this stops the reflex. But the reflex can be restored if the inhibiting fibre $G$ is also stimulated along with the other two, since it stops the activity of the inhibiting neurone, and leaves the neurone $\mathrm{A}$ free to convey the exciting impulses from E. Thus an inhibitory nerve may appear to start a reflex.

Since the two processes of excitation and inhibition are opposite, it is possible, stimulating them both in appropriate strength, to make them mutually abolish one another, so that no effect results. This can be shown in the case of certain muscles of the thigh, which are caused to contract when a particular afferent nerve of the opposite side is stimulated, and to relax if the corresponding nerve of their own side is stimulated. By different relative strengths of the two stimuli, various intermediate states between full contraction and relaxation can be brought about. Similar phenomena can be observed in the case of the nerves to the heart and the blood vessels, about which more will be said in the next chapter.

An important aspect of muscular movements is that known as reciprocal innerzation. Suppose that a limb can be either bent or straightened by the action of muscles, which are therefore antagonistic in their effects. It is found that when a reflex or voluntary movement involves the contraction of one set, the antagonists are concurrently relaxed by inhibition of their motor centres. It is clear that for the exact performance of delicate movements, such as those of the eye and the hand, the relaxation of the antagonistic muscles must proceed step by step with the contraction of the muscles producing the movement, and that, by this means, a very accurate adjustment of the movement can be made. 
Inhibition plays a very important part in the functions of the higher nerve centres, as we shall see presently. It is a matter of every-day experience that we can stop a movement suddenly, if necessary. This is effected not only by bringing antagonistic muscles into play, but by inhibition of those producing the movement which is required to cease.

\section{Fatigue}

If we perform the same movement many times in succession, it is possible to arrive at a state in which we cannot make the muscles contract any more. We say that they are fatigued. But we must be careful to distinguish this state from one of exhaustion. The store of material yielding energy to the muscles has not been used up, since an electrical current applied to them directly produces vigorous contraction. Something has happened at a synapse in the course of the nervous arc by which it ceases to be able to conduct. It may be either the using up of some material in this situation, or the production of some chemical substance that has not been removed with sufficient rapidity. But, in any case, the synapse recovers very quickly, and the fact of fatigue has the useful function of preventing the possession of any particular final common path by a reflex for an undue time. But why do we say that it is the synapse and not the whole neurone that is fatigued? It is because of the remarkable fact that the fatigue of a reflex arc, using a certain final common path as motor neurone, does not affect the use of this same motor neurone by another kind of reflex. The motor neurone itself seems to be very difficult or impossible to fatigue. It is also impossible to fatigue nerve fibres, except in the absence of oxygen, and even this has not been altogether satisfactorily demonstrated.

Fatigue of voluntary muscle itself, such as can be brought about by prolonged direct stimulation of excised muscle, is undoubtedly due to the accumulation of lactic acid, which is not oxidised as quickly as it is formed. If the muscle so fatigued is placed in pure oxygen, it recovers to a notable degree as the lactic acid disappears. But recovery is not complete, so that we must admit a partial exhaustion of the store of potential energy, due to inability to replace it in these abnormal conditions. The muscular fatigue of normal exercise is doubtless due in part to some excess of lactic acid. We know that in vigorous exercise the lactic acid is not oxidised completely as rapidly as it is formed, since some of it diffuses into the blood and is excreted in the urine. 


\section{The Cerebral Cortex and Conditioned Reflexes}

We may, from the standpoint of physiology, regard the responses in which the higher parts of the brain take part as a particular kind of reflexes. But they are more modifiable by effects influencing them by way of other parts of the nervous system than the machine-like spinal reflexes are. Thus, the contact of the hand with a hot object is always followed by withdrawal of the hand, but not necessarily by the use of "strong language." Here we see the intervention of inhibitory processes, which play so great a part in the functions of the cerebral hemispheres. The surface of these organs, known as the cortex, is the seat of the highest intellectual activities. In the investigation of its functions, a method has been developed by the eminent Russian physiologist, Pavlov, in which that aspect referred to above, namely, their variable nature, has been made use of in a systematic manner. Although a detailed account is beyond the scope of this book (see P., pp. 502-507), a brief consideration will help towards a general comprehension of the mode of action of the central nervous system.

This system has often been compared to a telephone exchange, and the resemblance is in many ways a striking one. Any one subscriber can be connected up with any other subscriber, just as a particular muscle can be used in reflexes from many various receptor organs. In this way the line to any one subscriber may be regarded as analogous to the final common path in relation to all other subscribers, and the costly and ineffective method of having this person separately connected by a special wire to each of the others is avoided. It is also possible for a subscriber to be permanently connected by a separate wire to another, so that these two can talk at will without having to be put into communication through the central exchange. This represents that kind of reflex with which we are familiar in the spinal reflex, but which is also to be found in parts of the brain intervening between the spinal cord and the cerebral cortex. It may be called unconditioned, because no special conditions need be present for it to be manifested. But the usual method is for a subscriber only to be temporarily connected with another, and the possibility of any resulting conversation depends on this condition. The contrast between the conditioned reflex, as it is obtained from the cerebral cortex, and the unconditioned one of drawing away the hand from a hot object, may be illustrated by supposing that one agrees with a friend to meet at a certain place at a certain time. We expect to do so under the conditions arranged. But a subsequent passing by the same place is not expected to have the same result. The association, as we may call it, is merely a temporary one. 
And in the use of the word "association" it is to be understood that we imply that an actual physiological process of connecting up in some way has taken place. It is clear that in such more or less complex activities of the higher centres there is a temporary functional union of neurones, which are not joined up in the ordinary course of affairs.

In the actual investigation of such processes, a reflex to the salivary glands in the dog was chiefly made use of. Although the fact has not been specially referred to, it is scarcely necessary to remark that any organ supplied with nerves which set it into activity may be so activated by a reflex from sense receptors. We saw in our discussion of digestion that the presence of food in the mouth almost invariably results in a secretion of saliva. This is the more primitive, unconditioned reflex. But it was also found that many kinds of external phenomena could, by appropriate means, be made to result in such a secretion, through the intervention of the higher centres, although these stimuli had previously no relation to food. Such a temporary association could be formed in the following way:-Food actually given to a dog produces secretion of saliva. Suppose that every time that the food is given, a particular bell is rung. After a number of repetitions of the combination of bell and food, a new connection has been set up between the sound of this particular bell and the presence of food, so that now the sound of the bell alone, which previously had no effect of the kind, excites secretion of saliva. This simple form of conditioned reflex allows many experiments to be made on the effect of various concurrent stimuli. The important part played by inhibition becomes very obvious. If during the production, or education, of the reflex to the bell some other extraneous stimulus intervenes, that to the bell is for a time obliterated. It can be shown also how the formation of the higher response overpowers the more primitive one. The application of an electrical current, strong enough to excite signs of pain, to a particular spot on the skin is made the signal for food, in the same way as the sound of the bell in the preceding experiment. After a time its application results in secretion of saliva in the absence of food, and under these conditions no signs of pain are shown. Whereas, if moved to a spot of skin a short distance away, the same electrical stimulus causes pain but no saliva. Such an experiment as the following has several points of interest. It was noted above that an extraneous influence is apt to prevent the manifestation of the proper conditioned reflex. So that if we have a secretion to the sound of a bell, a flash of light produced at the same time inhibits the reflex. Now, in the production of the reflex to the bell, suppose that the food is not presented at the same time as the 
sound, but two minutes afterwards, the process being repeated until the conditioned reflex is duly formed. We then find that the sound of the bell is not followed immediately by secretion of saliva, but only after two minutes have elapsed. It is obvious that something in the nature of an inhibition must have been going on during these two minutes. That this is the case can be shown by the application of a stimulus in this interval, the stimulus being one that does not produce secretion of itself, but has an inhibiting action on other stimuli, such as the flash of light above mentioned. The effect of this indifferent stimulus in the interval of two minutes before the secretion normally appears is to cause the appearance of saliva at once. The previous inhibition is itself inhibited, so that a positive result shows itself.

The inhibitory influences are spread over a wide area of the cortex ; in fact, during a conditioned reflex it appears that practically the whole of the cortex, with the exception of the part concerned, is in a state of inhibition.

We may conclude with one more example. Suppose that a sound and a light are made, each for itself, signals for secretion, but that when both are presented together no food is given, so that the reflex to the two stimuli together becomes one for no secretion, and one stimulus must inhibit the other. If one of these be afterwards presented alone, secretion follows, and if, while the secretion is in progress, the other, also active by itself alone, be superadded, the secretion stops.

It will be seen that we have in these new associations the physiological basis of memory and of the formation of habits, together with the possibility of their loss by breaking of the connections.

The fact must not be passed over that we have in the cortex certain areas whose artificial stimulation causes definite movements. These are called motor areas, but it must not be supposed that they are of the same nature as the motor neurones of the final common path. They may rather be looked upon as the physiological representatives of the ideas of particular movements, although their activity is not necessarily associated with consciousness, since the phenomena are shown in the anæsthetised animal.

The results of artificial stimulation of such cortical areas show the complexity of the various effects produced at different times by stimulation of one and the same point. Thus, after rest a point usually gives contraction of the muscle in the same way as it had previously, but if it be stimulated immediately after a previous response, inhibition of the muscle occurs. If a point which normally gives extension of the elbow be stimulated immediately after that of another point which gives flexion, the former point gives flexion 
instead of extension; and so on. Inhibition is more prominent than excitation, and appears independently of excitation of antagonists. After-actions, such as tonic or rhythmic contractions, are of various kinds, and affect the pairs of antagonists in a diversity of ways.

\section{The Nerve Impulse}

As pointed out above, there is nothing to be seen in a nerve fibre to indicate that a propagated disturbance is passing along it. Moreover, only one physical or chemical accompaniment of the impulse has been definitely shown to be present, that is, an electrical change of such a nature as to indicate that a point in a state of activity is electrically negative to one at rest. It has been stated that an evolution of carbon dioxide occurs, but the experiments are not altogether free from objection. The most sensitive instruments have failed to show that any evolution of heat takes place, and the absence of fatigue under normal conditions, referred to previously, indicates an extremely small consumption of energy. Indirect evidence suggests that what happens is a concentration of ions of a certain sign at or near some membrane, and that this concentration progresses as a wave along the fibre, the change at a forward point being brought about by the electrical effect of that behind it. The ions thus move backwards and forwards at any particular point, somewhat as the molecules concerned in the propagation of sound waves do.

Like other excitable tissues, nerve fibres exhibit a refractory phase, at first of loss of excitability altogether and then of gradual return to normal, or for a moment slightly beyond it. The whole period is very short, $0.0025 \mathrm{sec}$. in the frog, for the period of inexcitability. In man, it is propably about one-fourth of this value.

The rate of conduction of the impulse in man is about 120 m. per second.

We have seen that muscle fibres are only able to manifest one degree of activity, however the strength of the stimulus is varied. As far as motor nerves are concerned, the same fact of "all-ornothing " has been found, and, in all probability, it holds for afferent nerves also, since no other difference between the two kinds of nerve fibres has been detected.

The fact just mentioned is difficult to reconcile with a wave-like displacement of ions or similar view of the nature of the nerve impulse. If a nerve impulse passes through a region subjected to the action of an anæsthetic, it may be abolished; but if the anæsthesia is not too deep or the length anæsthetised not too long, 
it may be merely reduced in intensity. In the latter case, when it reaches a normal place again, it returns to its original strength, since it requires just as severe a treatment to abolish it completely as the normal impulse does. Such behaviour reminds one rather of that of a train of gunpowder which is very narrow in one part. If set alight at one end, the evolution of energy decreases as the chemical reaction passes along the narrow part, but it recovers again to its original value when it arrives at the part of the same width as the initial part, whereas a physical change, such as a sound wave, does not recover its original intensity after having been diminished by passing through cotton wool. On the whole, it cannot be said that the nature of the nerve impulse is yet solved.

\section{The Visceral Nervous System}

Those organs and tissues composed of smooth or involuntary muscle, such as the contractile coats of the intestines, heart, blood vessels, and so on, receive a nervous supply which differs in several ways from that of voluntary muscle. In the first place, as we have seen, there are both excitatory and inhibitory nerves; and, in the second place, these nerves are in reality the axons of association neurones and belong to the central nervous system, since they form synapses with a further set of neurones outside the nervous system, sometimes situated in the organ supplied, sometimes in masses of nervous tissue, ganglia, distinct from these organs. It is the axons of these neurones that pass to the actual tissue cells. In this system are also included fibres which go to secretory glands as well as to muscle.

A definite set of these visceral fibres is known as the sympathetic nervous system, and arises from a limited region in the middle part of the spinal cord. Some of these fibres supply smooth muscle, others glands, but all of them have the remarkable property of being set into activity by the secretion of two ductless glands at the upper ends of the kidneys, the supra-renals or adrenals. The agent responsible for this effect is known as adrenaline, and has been separated in the pure state.

Although the viscera have sensory nerves also, it should be noted that these nerves are similar in their nature and anatomical relations to the ordinary sensory nerves, so that the involuntary nervous system of the special nature described above is efferent only. 


\section{TRANSPORT OF MATERIALS-THE VASCULAR SYSTEM}

IN unicellular animals and in the more primitive small multicellular animals there is no need for the provision of special means of conveying chemical products from one part to another, since they readily pass by diffusion. But when, for example, the materials derived from the digestion of food are prepared in one particular part of the organism at a distance from other parts requiring them, special channels and means of transport are needed, just as we saw was the case with oxygen. And, as in that case, the means of transport is the blood. We have now to inquire how this transport is effected. It is clear that the blood must be sent in a current, so that its constituents may reach all organs, and that the same blood must circulate since there is no loss of it.

At a very early stage of evolution we find a muscular tube which, by rhythmical contractions, causes currents of a more or less irregular nature in the liquid of the body,cavity. This tube is open at both ends, but may be regarded as a rudimentary kind of heart, although the fluid which it drives is not confined to any particular channels, such as we find in the blood vessels of the more highly organised animals. In its most perfect form, as in the mammals, the general arrangement may be represented as in the diagram of Fig. 7. In this figure, for the sake of simplicity, the hollow muscular organ, known as the heart, is represented as two separate organs, left and right. Although the two parts are united in one mass, their cavities are quite distinct and separate. Starting, then, from the left side of the heart, at the upper right-hand corner of the diagram, we note that blood, which has replenished its oxygen and got rid of a large part of its carbon dioxide in the lungs, enters the contractile cavity, known as the left ventricle. But immediately before it enters the ventricle it passes through another chamber, the auricle, with thinner walls, but also contracting rhythmically, immediately before the ventricle. By this means the ventricle is filled up with blood. This ventricle then contracts with force, and as there are valves between it and the auricle which 
open only in such a direction as to allow blood to flow from auricle to ventricle, and not in the reverse direction, the contents of the ventricle are expelled into the main arterial channel, the aorta. Another set of valves is necessary at the beginning of this tube,

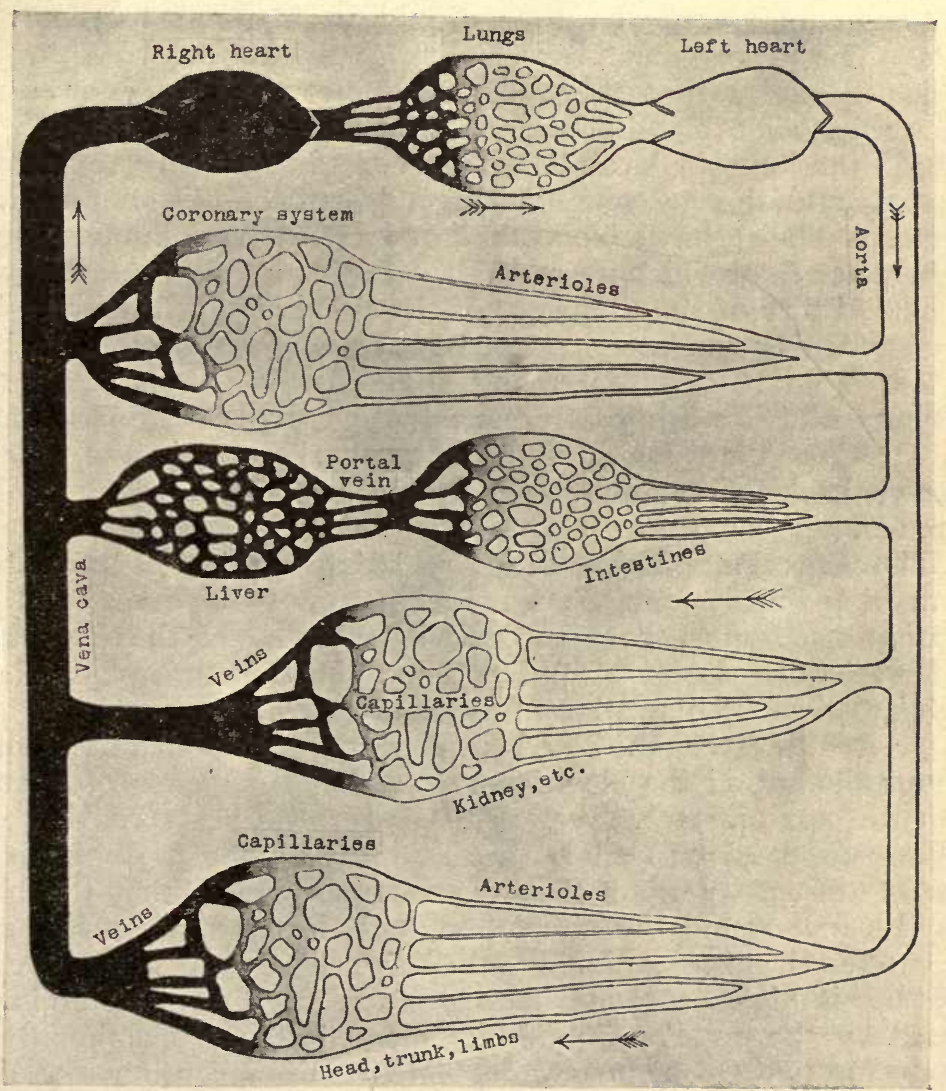

FIr. 7.-Diagram of the Vascular System of the Mammal.

in order to prevent the blood driven in, and stretching its walls, from flowing back again when the ventricle relaxes in preparation for another beat. The aorta gives off a large number of tubes, the arteries, of which four are represented in the figure. Each of these again divides into smaller vessels also with muscular walls, the arterioles, and these are continued into a network of minute connected tubes arranged around the tissue cells. These are the 
capillaries. The blood is collected again by small vessels, veins, which join to form larger ones, and then open into the main venous channel, the vena cava. The veins have thinner walls than the arteries, but are to some extent muscular. The vena cava carries the blood, which has by now given up a large part of its oxygen to the tissues and received carbon dioxide from them, to the right heart. The arrangement here is similar to that described for the left heart, except that the walls are not so thick and powerfully contractile. The blood driven out from it by a large artery passes through the lungs. A capillary system is formed here around the air sacs, and the gaseous exchange described in Chapter II. is effected. The restored blood then arrives by the pulmonary veins at the place from which we started.

The history of the discovery of the circulation of the blood is a very interesting one (see P., pp. 668-669). The real proof was given by Harvey in 1616 , although, in the absence of the microscope, it was impossible for him to see the actual passage of the blood from the arteries to the veins through the capillaries. This was done by Leeuwenhoek in 1686 , by means of the microscope which he had invented.

The gencral mechanics of the circulation can best be understood by making experiments on a model (E., p. 2I8). The actual circulation itself must be examined in the web of the frog's foot under the microscope (E., p. 22I), and the structure of the heart with its valves by dissection of a sheep's heart (E., p. 222).

We see then that the blood is sent through a number of channels in multiple arc, as the electrician would say, by means of a pump with the appropriate valves. This pump, the heart, consists of a hollow space surrounded by muscular walls, which diminish the size of the cavity when they contract. In order that the blood may be sent round the circulation, it is clear that a pressure must be produced, since a liquid only flows from a place where the pressure is higher to one where it is lower. This pressure is produced by the heart, which drives blood into the elastic arteries, producing tension in their walls. This tension continues to drive the blood onwards during the interval between the beats, so that the general arterial blood pressure does not fall greatly in this interval.

The way in which the supply of blood to different parts is regulated in accordance with their needs will be described later, but it can be seen from Fig. 7 how the widening of one alternative channel will result in a greater supply of blood to that part with a diminished supply to other parts, while a narrowing of it will result in a diminished local supply, with a greater one to other parts.

The uppermost of these parallel channels in the figure represents that of the heart muscle itself. As would be expected, the 
continuous work done by the heart requires a copious supply of oxygen and, in fact, a large part of the blood sent out by the beats of the heart is used to feed itself. The next channel in the figure is that through the stomach, intestines, and their attached glands. We notice the fact, to which attention has been directed above (p. 75), that the venous blood from these organs does not pass at once to the great veins, but traverses the liver on its way. Thus products of digestion are subjected to the action of this organ. The liver would thus receive only venous blood, except that provision is made for its oxygen supply by a special artery which proceeds directly to it. The third of the parallel paths represents that of the viscera, whose venous blood does not pass through the liver, and must be imagined to be itself composed of a number of separate channels. Similarly, the lowest one represents a large number of separate parallel paths.

\section{The Blood}

The blood is a liquid consisting of an immense number of tiny corpuscles suspended in a clear liquid, the plasma. These corpuscles are of two kinds, the red ones containing the hæmoglobin, which we have seen to be responsible for the carriage of oxygen and carbon dioxide; and colourless ones, like small amœbæ, which are in much smaller number than the red ones, are called leucocytes (E., p. 223). The chief function of the latter cells is to take up, kill, and digest disintegrating tissues, such as the tail of the tadpole when the frog stage is reached, and also the micro-organisms which invade the body and cause disease. The process is known as phagocytosis, although it is just like the ordinary feeding of the amœba. It seems probable that these corpuscles may have other functions, but very little is known of these.

The red corpuscles, as already mentioned, consist almost entirely of hærnoglobin, together with water. In vertebrates other than mammals they possess nuclei, and have the general properties of living cells. In mammals they lose the nuclei which are present when the corpuscles are young. It was pointed out in our first chapter that living cells when deprived of their nuclei degenerate and die, so that it is a matter of some difficulty to know whether the red corpuscles are actually to be regarded as living or not. At any rate, they disintegrate after a certain number of days. The destruction takes place almost entirely in the liver, and the bile pigments are formed from their hæmoglobin. These pigments contain no iron, that present in the hæmoglobin being taken up by the liver cells.

New corpuscles are formed in the red marrow found inside a 
number of bones. Apparently the iron from the old corpuscles is utilised in the process, being conveyed from the liver to the marrow in some way. It might seem a somewhat remarkable situation for this important process, but the reason seems to be that the channels for the blood must be sufficiently thin-walled and delicate to permit the newly-formed corpuscles to pass into them, and the protection afforded by the solid bones is advantageous.

The plasma contains two or three different kinds of proteins in colloidal solution, together with various salts, of which the chief is sodium chloride, organic foodstuffs, such as glucose and aminoacids, and waste products, such as urea, in small amount. It also contains fat in very finely emulsified form. The proteins do not serve as food materials. Certain of them take part in that kind of "setting" of blood into a jelly, which is called "clotting," and will be referred to again presently. Other functions of the proteins will be better understood later. The salts are necessary in connection with the maintenance of the correct properties of the cell membranes. Without them the functions of the living cells come to an end.

Internal Secretions. - We have seen that food materials, including oxygen, are supplied to all the tissues through the medium of the circulating blood, and that the carbon dioxide produced by oxidation is removed. Further, the various chemical waste products are carried away to be got rid of through the kidneys. But we have also seen that amongst these chemical products there are certain substances, made by glandular secreting organs, which substances pass directly into the blood and have powerful physiological effects on various organs and tissues. In many cases these substances are essential to life, so that disease or removal of the organ producing one of them results in death from various morbid conditions. Such are the thyroid gland in the front of the neck, the suprarenal glands above the kidneys, and others. The absence of the normal thyroid gland leads to a swollen state of the tissue under the skin, to mental deficiency and other abnormal symptoms. The absence of the suprarenals results in what is called "Addison's disease." The absence of certain cells in the pancreas leads to diabetes, and so on. In other cases, as in that of the "interstitial" cells of the sexual glands, ovary and testis, profound changes depend on their internal secretions. Details of the numerous organs of this kind will be found in the larger text-books, but we see that there exists an extensive series of substances which act as chemical means of co-ordination between different parts of the body, and have been called "chemical messengers" or "hormones." It seems not unlikely that every kind of tissue produces some substance of this kind, but it is clear that we cannot remove the whole 
of the muscles, for example, in order to discover whether this is the case with them.

The most typical of all chemical messengers actually obtained in solution is the "secretin" produced by the action of acid on the lining cells of the first part of the small intestine. This is formed for the express purpose of setting the pancreas into activity, as we have seen.

Chemical messengers are also to be met with in plants. There are, as it appears, chemical substances which diffuse from some parts and, circulating in the sap, favour the growth of other parts. But these may possibly be of the nature of food materials. There are others, however, which check or inhibit the growth of certain parts in a definite manner. Thus, the apical shoot of a fir tree produces some substances that prevent the lower shoots from growing vertically upwards; so that when this shoot is cut off or injured, one of the lower ones which is growing more vigorously than its neighbours begins to grow vertically. As it grows upwards, it, in its turn, produces the inhibiting material and prevents others from turning upwards. It would appear that the exciting cause must be the action of gravity on some constituents of the cells, analogous to starch grains in the more common form of response to the stimulus of gravity.

The Kidneys.-In addition to carbon dioxide, there are other waste products of tissue activity. The most important of these is urea, resulting from the deamination of amino-acids in the liver. There are also other compounds of nitrogen, arising either as byproducts of chemical reactions, or from wear and tear of cell structures. Amongst these are uric acid and creatine. Then again, there are substances taken in with the food, which are either useless or only wanted in small quantity, such, for example, as phosphates and sulphates. We notice that all these substances which we want to get rid of are crystalloids of small molecular size, present in true solution, so that if we filter the blood through a membrane which has pores small enough to prevent the passage of the blood corpuscles and the colloids, we can effect a separation. Such a membrane is parchment paper, as we saw in the first chapter, and the wall of the small blood vessels is a membrane of similar properties as regards permeability. But in filtering off the waste products in such a way, we remove from the blood other constituents which are of value, namely, water, salts, glucose, and amino-acids. Nevertheless, this is the method adopted by the animal body to rid itself of its waste products. How the disadvantage is remedied will be seen later.

The kidney is the organ in which the process takes place, and the structures in it where filtration occurs are coils of small blood 
vessels, "glomeruli," suspended in cavitics which are themselves the beginnings of long-looped and twisted tubes, lined with cells, along which the filtrate passes in its way to the duct, called "ureter," which conducts it to the bladder. As the filtrate from the glomeruli passes along the "tubules," it is subjected to certain operations which convert it into urine, the name given to the liquid which leaves the kidney. It is to be understood that the glomeruli and tubules are in very large number, so that a great area of surface is provided for filtration and the subsequent operations (E., p. 223).

Fig. 8 represents, diagrammatically, what takes place in the glomeruli.

In order that the filtration referred to may take place at a perceptible rate, the liquid to be filtercd, the blood, must be subjected to pressure. The heart provides this pressure in the arteries. Now it is found that if the arterial pressure is lower than about 40

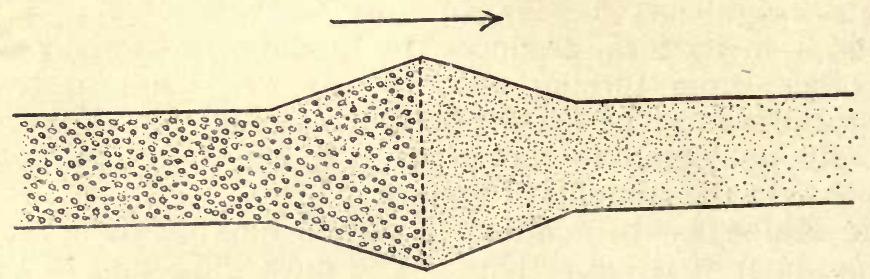

FIG. 8.-Diagram of the Filtration in the Glomeruli.

The clear space represents water.

The dots, the crystalloids.

The small circles, the colloids.

mm. Hg. no filtration takes place at all. Why is this? We saw (E., p. 172) that the colloids, almost entirely proteins, of the serum possess an osmotic pressure of about $40 \mathrm{~mm}$. of mercury. This means that they attract water through a membrane impermeable to them, unless the entrance of the water is opposed by a pressure of $40 \mathrm{~mm}$. Hg. If the pressure is greater than this, then water, containing in solution any substances to which the membrane is permeable, is filtered through at a rate proportional to the height of the filtration pressure above $40 \mathrm{~mm}$. Hg.

The glomerular filters are able, then, to get rid of waste products without any other mechanism; but, as remarked above, with the loss of large quantities of water, a matter of great importance to land animals, together with solutes which are of value, especially sodium chloride, glucose, and amino-acids. In marine animals the loss of water and of sodium chloride would not matter; but even here that of food materials is more serious. Accordingly, we find 
developed in the cells lining the tubules of the kidney a capacity for reabsorbing water and its valuable solutes, leaving untouched the waste products. In other words, a solution is reabsorbed containing all the normal constituents of the blood plasma, except urea and such substances as are not wanted. Of course, the liquid which is reabsorbed does not contain proteins, because they are absent from the glomerular filtrate. Now, it has been pointed out by Professor Cushny that all the various phenomena connected with the formation of urine can be most simply and easily explained if we suppose that the solution reabsorbed by the tubule cells, and passed back to the blood, is that to which the mechanism of these cells, together with the other cells of the body, is adjusted. That is, a solution which contains not merely the normal salts and food materials of the blood, but also in the exact concentration which they possess in the blood. Such a fluid then is invariably absorbed, whatever the composition of the glomerular filtrate passing over the cells. If the filtrate contains a notable excess of some solute, even of a valuable constituent such as sugar, it may happen that a part of it is lost by failure to be absorbed. Moreover, the rate at which absorption is possible is limited, so that if filtration is very rapid, there may not be time for absorption to take place as perfectly as under normal conditions.

In order to see how the process works, let us take the case in which the blood has been diluted by drinking a large quantity of water. In the first place, the colloids will be present in lower concentration than normal, hence the available filtration pressure is raised. The filtrate will, however, be more dilute than normal, while the tubules absorb from it a solution of the normal concentration. The result is that the concentration of the blood is more or less quickly raised again.

Suppose next that the blood has become concentrated by loss of water from the skin. It is obvious that the filtration will be slower, but a certain amount of water must be lost in order to kcep in solution the excretory products. The liquid absorbed is of normal concentration, so that the blood does not become so concentrated as it would otherwise, while the urine may be of very high concentration.

Although the glucose and amino-acids are practically completely reabsorbed, there is always a loss of sodium chloride, which is replaced by fresh supplies in the food. And, as already remarked, if abnormal amounts of the former materials are present in the blood, we find them in the urine. If the liver is disordered, so that the deamination of amino-acids is interfered with, we find these acids in the urine.

Since the filtration pressure in the glomeruli is the factor con- 
trolling the rate of formation of urine, it is clear how this rate can be modified apart from the effect of dilution of blood. If the small arteries conveying blood to the filters are narrowed, by contraction of their muscular coats, the pressure is reduced and the rate of filtration decreased. And conversely, if they are dilated, the rate goes up. Moreover, changes in the main arterial pressure will have the same effects, independently of local changes in the renal circulation. There are means of bringing about these various changes by reflexes from the nervous system, as we shall see presently.

Lymph.-There is no reason to suppose that the blood vessels of the glomeruli differ essentially in the nature of their permeability from those of the rest of the body; in fact, we have direct evidence that the blood vessels generally are permeable to water and crystalloids, impermeable to colloids. Hence, it may be asked, do they not in other places than the glomeruli allow protein-free filtrate to escape into the tissues, and, if so, what becomes of it? This, indeed, is actually the case. As the blood flows from the arteries through the capillaries to the veins by virtue of the greater pressure in the former, in a part of its course the pressure is greater than the osmotic pressure of the proteins. Liquid is filtered out here, and is known as "lymph." It is the part of the blood with which the tissue cells are in immediate relation. As the blood current passes onwards to the veins, where the pressure is very low, at a certain region the pressure has fallen to a value equal to that of the osmotic pressure of the colloids, and beyond this point the internal pressure in the capillaries is lower than the osmotic pressure of the colloids. Accordingly, this osmotic pressure becomes active here in attracting water, so that the lymph which was filtered off in the previous part of the course is, to a large extent, reabsorbed. Although the area in which reabsorption occurs is probably larger than that in which filtration occurs, the rate of reabsorption is insufficient to remove the whole of the filtrate, and what remains passes away in channels which commence in the spaces between the cells and gradually become definite vessels with thin walls, finally joining together to form a large vessel, the "thoracic duct," which opens into the veins at the root of the neck.

Since it is by the osmotic pressure of the colloids in the blood that the water is prevented from escaping into the tissues, and causing what is known as "œdema," we see the object of adding a colloid, such as gum arabic, to a liquid used for intravenous injection to replace blood lost, or increase the volume in actual circulation. Solutions containing crystalloids only have been found useless, since they rapidly escape from the circulation. Whereas, if 6 or 7 per cent. of gum be added, they remain in the circulation, maintaining the volume and pressure of the blood at 
their normal heights. Such solutions were found of great service in the treatment of wounded men in the late war.

We saw previously that fat is absorbed in the intestine by passing into lymphatic channels.

In part of their course the lymphatic vessels pass through what are called "lymphatic glands." These are ductless glands, which supply small leucocytes to the lymph, and thus to the blood. They sometimes make their existence known by becoming inflamed and painful when the tissue from which the lymphatics passing through them arise is in a state of disease and giving off poisonous substances to the lymph.

In some situations the wall of the blood vessels appears to be more permeable than is the rule. Thus, in the liver, proteins pass through slowly. Such an increased permeability may be conferred on the blood vessels in other parts by the injection of certain substances, such as an extract made from dried mussels. It happens also in the remarkable state called "anaphylactic shock." As mentioned in our second chapter, many diseases are due to poisonous substances given off by bacteria to the blood. These are normally counteracted, or made innocuous, by the production of "anti-bodies" in the blood, which act upon the foreign poisons in various ways. A similar reaction occurs when proteins other than those of the animal's blood are introduced. But to produce this "immunity" in a marked degree requires the injection to be repeated several times with certain intervals. If, however, the second injection is delayed for more than ten days or so, it is found that a greatly increased sensibility is produced, so that the injection is followed by serious collapse and fall of blood pressure. One of the symptoms present is frequently a swelling of the subcutaneous tissue due to escape of liquid from the blood. If the animal recovers from this state, it is found to be "desensitised," that is, any further dose is innocuous. Satisfactory explanations of these complex phenomena have not yet been given.

The Proteins of the Plasma.-We may now add a little more with respect to the function of these constituents. They do not act as food; the tissue cells require amino-acids. They are, apparently, the source of the anti-bodies spoken of in the preceding paragraph. The two most important properties that they possess are their osmotic pressure, by which the blood is prevented from losing water and the tissues from becoming water-logged, together with that of clotting. This is a process, involving several of the proteins of the plasma, which occurs when the blood comes into contact with most foreign substances, if they are wetted by it. Clotting consists in the separation of a solid, "fibrin," in the form of a network of filaments in which the corpuscles and liquid of the 
blood are at first entangled. Liquid slowly exudes, owing to the contraction of the network. This liquid, of course, is not the same as the plasma, since fibrin has been separated from it, and other products of the reaction which results in the deposition of this solid are left in solution. The liquid is called "serum." The importance of the phenomenon is in the spontaneous arrest of bleeding from an injured blood vessel. If the rate of the outflow is not great, the issuing blood clots when it comes into contact with the tissues, and the familiar effect of accelerating the process by the application of cobweb or such-like is merely due to the provision of a large area of foreign surface.

The precise explanation of the changes that take place in this coagulation process has led to various theories which it would not be profitable to discuss here. It is to be feared that much of what has been written on the question amounts to little more than inventing names. There is one important fact, however, namely, that the blood remains liquid if calcium be removed from it, as by the addition of the appropriate amount of an oxalate, which forms the insoluble calcium oxalate.

The Salts of Blood.-We saw in our second chapter (p. 6o) that the cells of the present land animals have become adjusted to the presence of certain salts, probably owing to their presence in the ocean at the time when their ancestors left it. In order, therefore, that a saline solution may serve as a perfusion fluid for isolated organs, it has been found that particular salts must be present, and in a "balanced," relative concentration. These salts are those of sodium, potassium, and calcium, generally used as chlorides (E., p. 223). The function of the sodium chloride is chiefly to afford a sufficient osmotic pressure to balance that of the cell contents. Calcium seems to be necessary to maintain the properties of the cell membrane, but it has doubtless other functions as well. Potassium neutralises certain deleterious effects of calcium, and is also said to be of importance on account of its radio-activity, since it can be replaced by salts of other radio-active metals in equivalent radio-active concentrations. The electrical properties of the anions and cations naturally also come into play in the balance of electrolytes, but the problem is not completely solved.

Since the cell mechanisms are very sensitive to changes in the concentration of hydrogen ions, while acids are produced in the tissues under active conditions, a means of maintaining the hydrogenion concentration of the blood at a constant value is a necessity. The normal reaction is just about the neutrality of distilled water, very slightly on the alkaline side. Although the proteins of the plasma are able to combine with acids and alkalies, this capacity is limited and scarcely comes into play within the region of the most 
importance, that in the immediate neighbourhood of the neutral point. The osmotic pressure of the proteins in the plasma is about $40 \mathrm{~mm}$. of mercury, as we have seen, so that their molar concentration is only 0.0023 , and they do not possess many free $\mathrm{NH}_{2}$ groups capable of combining with acids. It appears that it is half the nitrogen of the lysine contained in the protein molecule that is in the form referred to. If so, the proteins of serum would be about equivalent to a 0.0006 normal ammonia solution.

But there is a salt present in plasma which can combine with acids. This is sodium bicarbonate, and its concentration is 0.03 molar. On investigation, solutions of this salt are found to be very effective in preventing a rise in the hydrogen-ion concentration of solutions when acid is added. This is because when an acid stronger than carbonic acid is added to a bicarbonate solution, carbon dioxide gas is given off to the atmosphere, while that remaining in solution is so little dissociated as to afford only a few hydrogen ions as compared with those of the acid added. We may look at the question from another point of view. Sodium bicarbonate solutions are alkaline because the salt, as one of a weak acid with a strong base, is hydrolytically dissociated into carbonic acid $\left(\mathrm{H}_{2} \mathrm{CO}_{3}\right)$ and sodium hydroxide. The latter is electrolytically dissociated much more than the former, so that there is an excess of $\mathrm{OH}^{\prime}$ ions, conferring alkalinity. Thus, in a solution of sodium bicarbonate containing dissolved carbon dioxide, we may say that the former confers alkalinity, the latter acidity, hence the hydrogenion concentration is given by the ratio between the two. If they vary in proportion, the reaction is unaltered. If the carbon dioxide increases without the bicarbonate changing, the hydrogen ion is raised. If it decreases, the hydrogen ion is lowered. These facts give the clue to the most rapid and effective of the means of regulating the reaction of the blood. Let us suppose that lactic acid has been passed into the blood, as happens in defective supply of oxygen. It immediately combines with a part of the bicarbonate, and if the content of the plasma in this salt is determined, it is found to be diminished. The state is often called "acidosis," although it does not imply that the blood has become more acid. In fact, it is easy to show, by experiment, that a large amount of acid may be introduced into the blood without raising the hydrogenion concentration. Why not? The answer is found in the activity of the respiratory centre. When bicarbonate combines with acid, carbon dioxide is given off and, for a brief period of time, the hydrogen ion of the blood is raised by its excess. But this excess excites the respiratory centre to increased ventilation of the lungs until the carbon dioxide tension in the alveolar air has become low enough to reduce that in the blood to a level to compensate for 
the reduced bicarbonate, thus bringing back the ratio of carbon dioxide to sodium bicarbonate to its normal value. A converse process accommodates to an increased alkalinity, although it appears to be less effective, perhaps because the requisite decreased ventilation implies a diminution in the supply of oxygen.

In addition to this method there are two other means of maintaining the neutrality of the blood. In the first place, an increase in the acidity of the blood causes, in some way, a retardation of the formation of urea from the ammonia resulting from the deamination of amino-acids in the liver (p. 77). This ammonia then neutralises acid, and the salt formed is excreted in the urine. It has recently been found that if excess of alkali is introduced into the blood, lactic acid is formed in the organism. This combines with the base present in excess, and the neutral lactate appears in the urine. In the second place, the kidney itself acts as a regulator. As the reader is doubtless aware, there are two phosphates of sodium or potassium; one of these $\left(\mathrm{NaH}_{2} \mathrm{PO}_{4}\right)$ is acid, the other $\left(\mathrm{Na}_{2} \mathrm{HPO}_{4}\right)$ is alkaline. A mixture of the two in certain proportions is neutral. They are contained in small amount in the blood in this latter ratio. If the blood becomes more acid, a larger proportion of the acid phosphate is formed. This passes into the glomerular filtrate. The phosphates in solution are hydrolytically dissociated, so that there are present sodium hydroxide and phosphoric acid. Sodium salts are wanted by the body, while phosphoric acid is only wanted in very small amount. The tubules, therefore, absorb the sodium in the form of bicarbonate, leaving the phosphoric acid in slight excess, so that the urine is usually slightly acid. It will be clear that if the phosphoric acid is in greater excess than normal in the glomerular filtrate, it will be left behind and escape with the urine, while a fluid of normal reaction will be absorbed into the blood.

Viscosity.--The molecules of a liquid experience friction in moving over onc another, so that if a part of the liquid is at rest while another part of it is in motion, there is friction between the two. This internal friction is the cause of that property known as viscosity, familiar to all in the difference between water and glycerin or treacle. If there are particles in the liquid, such as the blood corpuscles, the viscosity is greatly increased, partly owing to the fact that where a liquid is in contact with a solid, a film of it is held stationary, so that there is more friction in the whole mass than if the corpuscles were absent. Blood corpuscles are also deformed in shape when passing through narrow channels, and this serves to increase the apparent viscosity, since a part of the energy of the current is taken up in the changing of shape. Indeed, the viscosity of the plasma is only about half as much again as that of 
water, whereas that of the whole blood may be as much as four or more times that of water.

A high pressure in the arteries could only be maintained by the heart if there were resistance to the outflow through the small branches. This resistance is not due to friction between the blood and the walls of the vessels, because the layer in contact with the wall is stationary, but to that between successive layers of the blood itself, extending to some distance from the wall. In a small tube this distance is great enough to reach to the middle of the lumen; in a large one the greater part of the current may be all
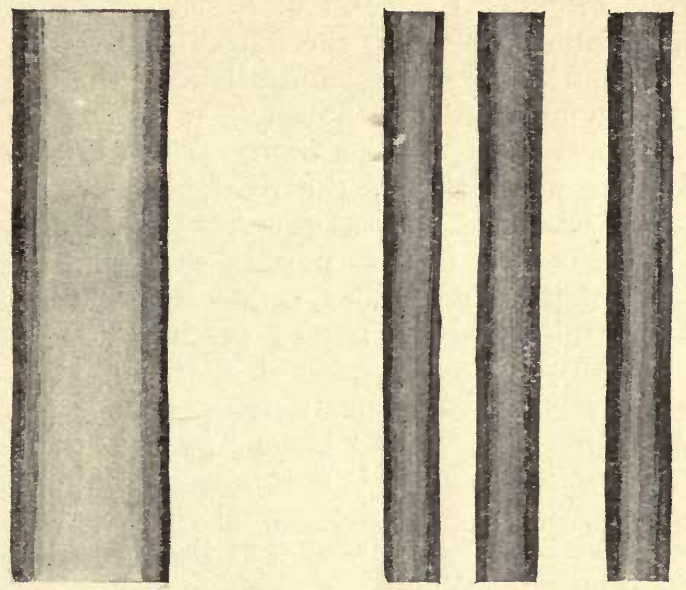

FIG. 9. - To illustrate the relative magnitude of the region where internal friction of the blood takes place in large and small arteries of equal total sectional area.

moving at the same rate, and experience no internal friction (Fig. 9). Hence the chief situation of the resistance is to be found in the fine branches of the arteries, the arterioles. A volume of blood flowing through a large tube experiences friction only in a small part near the walls. The same volume flowing through a number of smaller tubes, of the same total sectional area, experiences friction throughout its mass. We see how the peripheral resistance, and with it the arterial pressure, may be increased by constriction of the arterioles or decreased by their dilatation.

The Regulation of Blood Supply.-If the diagram of Fig. 7 be referred to, it will be realised that if all but one of the parallel channels be made narrower, this one will receive more supply; because if the heart continues to beat with the same strength, the 
driving pressure will be greater. This is one way in which the supply to any particular organ may be increased, and so far as investigation has hitherto been able to make out, it is the only way in which that to the brain is regulated, so far as other than chemical factors acting directly on the blood vessels are concerned. If the brain requires more blood, the whole of the rest of the body has to put up with less. In most organs, however, the arterioles have the power of widening in response to messages from the central ncrvous system, thus ensuring for each particular organ the more copious supply that is wanted when it enters into activity. There is thus a double supply of nerves to the muscle of the arterioles as to smooth muscle in general, one set of nerves exciting to increased contraction, the other inhibiting the natural tone. The former are called "vaso-constrictor" nerves, and all leave the central nervous system in the sympathetic outflow (E., p. 224). The latter are "vaso-dilator" nerves, and have a more various origin. A familiar case of reflex vaso-dilatation is the reddening of the skin known as "blushing." Each set has a governing centre, the source of reflexes to blood vessels, in that part of the brain immediately at the upper end of the spinal cord, called the "bulb" or "medulla oblongata." Certain sensory nerves produce, on stimulation, a reflex fall of blood pressure by general dilatation of the arterioles; others a rise by general vaso-constriction. The former are sometimes called "depressor" and the latter "pressor" reflexes.

The vaso-constrictor centre is normally sending out irnpulses in a continuous stream down the spinal cord and through the sympathetic to the blood vessels, so that these are kept in a state of partial contraction. When a depressor reflex is produced, the vaso-constrictor centre is inhibited, while the vaso-dilator centre is excited. In fact, we have "reciprocal innervation" of a rather more complex kind than in that of reflexes to voluntary muscles. The converse effect in a pressor reflex is not so easy to show, since the vaso-dilator centre does not send out a steady discharge except under special circumstances. The excitation of the constrictor centre is easily to be made out.

One particular reflex requires mention, that from the nerve which has received the special name of "depressor." The receptor endings are situated chiefly in the beginning of the aorta, so that when the blood pressure rises too high a dilator reflex is sent to the arterioles in general, and the pressure lowered.

Another kind of vascular reflex is met with in several organs, and is probably of wide occurrence. It is known as the "Lovén reflex," from the Swedish physiologist who first described it. When a sensory nerve passing from the rabbit's ear, for example, is stimulated so as to evoke a reflex to the blood vessels, it is found 
that the arterioles of the ear itself are dilated, while those of the rest of the body are constricted, so that a rise of arterial pressure results. It will be seen that this is the most effective means possible of obtaining a more copious blood supply to any organ.

The arterioles can also be made to dilate or constrict by the action of various drugs or chemical substances. The constricting effect of adrenaline has already been referred to. Some of the chemical products of active cells have a dilating effect, the chief of these being the hydrogen-ions from carbon dioxide. Hence an increase of activity automatically brings about a better supply of blood.

The Capillaries.-Although the walls of the fine network of blood vessels that connects the small arterioles with the small venules consist of simple protoplasmic cells and possess no muscular coat, there is evidence that these vessels can be made narrower or wider. This must occur owing to the constituent cells becoming thicker and less flattened when contraction takes place, somewhat as an amøba or leucocyte does when stimulated. Whether they are under the control of nerves is not yet decided, but certain chemical agents have been shown to relax them. A substance called "histamine," which is derived from a complex amino-acid constituent of protoplasm by removal of carbon dioxide, has a dilating effect on the capillaries, but a constrictor one on the arterioles. Under its action, therefore, an accumulation of blood in a nearly stagnant state is liable to take place in the capillaries, and such a condition, by which blood is removed from active circulation, is of importance in the condition of "shock" after wounds or surgical operation. Substances with an action similar to that of histamine would be produced in the destructive changes going on in damaged cells.

A condition in which the capillaries are dilated, but with very little blood current through them, owing to constriction of arterioles, is seen in the blue skin sometimes produced by cold. The fact that the skin is dark in colour, not white, shows that the capillaries in it are full of blood, but this blood is almost stationary. Its colour shows that oxygen has been removed, and the coldness of the skin shows that the circulation through it has nearly ceased. The skin may also be full of blood, but red and hot, as when exposed to warmth or heated by friction. In this case, the arterioles are dilated, and the capillaries are passively distended by the raised pressure thus produced inside them. Thus a full current of blood passes through them.

There is evidence that when organs are not in activity the whole of their capillary vessels are not filled with blood. Some are empty and more or less invisible. These may be filled with red blood when the arterioles dilate, or with venous blood when they themselves 
dilate without dilatation of the arterioles or with constriction of these latter.

The Regulation of the Heart Beat.-For the sake of simplicity of description of the mechanism of adjustment by changes in the blood vessels, we have supposed that the heart has continued to expel the same amount of blood in equal times. But the heart has its own powers of regulating its output of blood, and thus of maintaining a good arterial pressure even when the peripheral vessels are dilated, and of moderating it if there is much increase in peripheral resistance by vaso-constriction.

At one time this adjustment was thought to be of a very complex nature, but the work of Starling and his co-workers has resulted in reducing it almost completely to a comparatively simple "laze of the heart." It will be clear that the degree to which the ventricles are filled by the time at which they contract depends on the amount of blood which has flowed in during the pause between two beats. Supposing that the heart muscle always contracted to the same extent, then the fact that there was more blood in the ventricular cavity would not result in more being expelled. In fact, the opposite would occur, on account of the less mechanical advantage of the tangential force when the curvature is less, as it is in the more distended ventricle. When, therefore, the arterioles of an extensive region of the body dilate, as in the muscles in running, or the alimentary canal in digestion, the larger quantity of blood entering the heart from the veins would only be sent on incompletely, and the benefit of the vaso-dilatation would only imperfectly be realised on account of the large fall in arterial pressure. Moreover, the blood flow through the lungs would not be increased, and the urgent need for more oxygen would fail to be satisfied. In actual fact, however, this is not the case. It is found that the ventricles of the heart expel more blood per beat the more they contain to begin with, unless the initial distension is excessive.

Further, this behaviour is shown by the heart when separated from the central nervous system, so that it is due to some inherent property of the heart muscle itself.

Again, it is found that when the arterial pressure is raised, the amount of blood expelled by the heart in a given time is not decreased, although to raise the same volume to a higher pressure requires more work. How is this to be explained? Suppose that a particular heart is working with such an expenditure of energy that the arterial pressure is kept at a mean value of $80 \mathrm{~mm}$. of mercury, and that to do this 8 c.c. of blood are expelled at each beat. It is to be remembered that the pressure against which the heart works rises from about $65 \mathrm{~mm}$. at the beginning of the outflow to $100 \mathrm{~mm}$. at the end. The peripheral resistance may now 
be supposed to rise, so that a mean pressure of $140 \mathrm{~mm}$. is required to keep up a flow of the same magnitude as before, as actually takes place. The next heart beat will not be powerful enough to raise the pressure above, say, $100 \mathrm{~mm}$., but this will be done by the expulsion of less blood than before, so that a certain amount is left behind in the ventricle. Since the same amount as before flows in from the veins, the ventricle starts the next beat at a greater distension than that of the previous one, and, as we saw above, its contractile energy is greater. The increase continues with each beat until the original output is reached.

Now, these facts and various others relating to the automatic adjustment of the strength of the beat are readily and simply explained by that property of muscle to which attention has already been directed (p. 96). We saw that the amount of energy developed in muscular activity is in direct proportion to the length of the fibre during the time that it is in the act of developing its state of tension. This energy, in fact, is proportional to the area of certain surfaces arranged lengthwise in the fibre. A greater distension of the cavities of the heart is necessarily accompanied by a stretching of all the constituent muscle fibres of their walls, so that when contraction takes place it starts from a greater length of the active surfaces, and greater energy is produced.

There is also what may be called an external control of the force and rate of the heart beat. We have already seen that there are two characteristics of smooth muscle in general, and the walls of the heart behave as this kind of muscle, although they possess the transverse striation of voluntary muscle. The first of these is that of automatic activity, which may be manifested either in a state of moderate contraction or by a series of rhythmical beats, or both combined. This is very obvious in the case of the heart, which continues to beat when cut out of the body, and will require further consideration presently. The second characteristic is the supply of two kinds of nerves, one increasing the state of activity (excitatory), the other decreasing it (inhibitory). In the case of the heart thesc functions are exercised both on the strength of the beat and on its rate. The excitatory nerve fibres come from the sympathetic system, and are known as the "augmentor" or "accelerator" nerves. They may be looked upon as similar to the vaso-constrictor nerves, which we found to come from the same system. The inhibitory nerve fibres are contained in the vagus nerves, and have a general depressant action, not only on the strength and rate of the beat, but also on the excitability of the muscle to stimuli and on its capacity to conduct the wave of contraction. Both of these nerves can be set into action by reflexes. The depressor nerve, which we saw to produce a fall of 
blood pressure by vascular dilatation, also produces slowing of the heart through the vagus nerves.

The endings of both kinds of nerves in the heart are accessible to chemical stimuli. Thus the vagus endings are stimulated by acetyl-choline, the accelerator endings by adrenaline. The former are paralysed by atropine (E., p. 225).

Origin and Transmission of the Heart Beat.-If the heart of the frog or tortoise be observed carefully (E., p. 225), it will be seen that the different cavities contract in a regular order, beginning at the junction of the great veins with the sinus venosus, and ending at the commencement of the aorta. Thus there is a place which has the property of beating more rapidly than other parts. In fact, if the various cavities are cut away from one another, it is found that the natural rate of the activity of each part decreases in order of its distance from the sinus. Since the contraction of one cavity is transmitted to the next, it is clear that the most rapid one sets the pace for the others. In the mammalian heart, although the sinus no longer exists as a separate cavity, there is a mass of tissue of similar structure to the sinus, and situated at the point where the great vein of the head and neck joins the right auricle. From this tissue each heart beat is initiated, and it is known as the "Keith-Flack or sinu-auricular node." In the frog, the contraction progresses as a wave in the ordinary muscular tissue, which is continuous throughout the series of chambers. There is, however, evidence that even here there is a certain degree of specialisation of a part of the connecting tube or funnel between auricles and ventricle, such that this part conducts more rapidly than other parts. In the mammal, this conducting tissue has become a bundle of a peculiar kind of muscle cells which have developed the capacity of rapid conduction. Consideration of the anatomical arrangement by which the blood enters the ventricle at what is called the base, where it is united to the auricle, and also leaves it at the same end, leads us to realise that it must be an advantage, especially in large hearts, if the ventricle contracts as simultaneously as possible in all its parts, instead of in a rather slow wave progressing from base to apex. This is provided for by the auriculo-ventricular bundle above mentioned, which conducts about ten times as fast as the ordinary ventricular muscle, sending out branches to all the various regions of the ventricle. Thus contraction at the apex is almost coincident with that at the base, and the contents of the cavity are expelled more effectively than if different parts were not in the same phase of contractile stress at the same time.

The nerves which regulate the beat of the heart are in especially intimate connection with the node from which the contractions start. 


\section{CHAPTER VII}

\section{GROWTH AND REPRODUCTION}

IT is a somewhat remarkable fact that by far the larger number of the different species of living organisms have a certain size to which they grow, and individuals deviate but little from this size. One cannot make any general statement as to why this is so, but it seems likely that the causes are various, sometimes mechanical, sometimes, perhaps, due to the digestive arrangements being unable to supply a larger bulk with the necessary food. If a growing rat is supplied with a diet which is adequate to maintain it at a small size, but inadequate for growth, it may remain at this size long after it ought to have been fully grown. If, then, it be given a complete diet, it grows to the normal size, but not beyond it.

Similarly, it is not easy to say why an organism should, sooner or later, cease to perform those functions which we call "life," die and disintegrate. It is to be presumed that some essential part of the cell machinery cannot be replaced when it has worn out, although this conception does not lead us far.

In any case, the fact of the death of the individual makes it necessary that provision be made for the continuance of the race in new individuals - for the production of a young and vigorous new generation by what indeed we know as "reproduction."

In such lowly organisms as the bacteria, we find that, when an individual has grown to a certain size, it simply divides into two, and the process goes on at a great rate under favourable conditions. When supply of food is limited, or the medium in which they are growing dries up, a part or the whole of each individual organism collects into a mass and becomes surrounded by a layer of resistant material, apparently almost impervious to water. These are called "spores." In this condition, bacteria are much more difficult to kill by heat. They remain dormant, but become active forms again in the presence of water and food. It is not, however, all kinds of bacteria that form spores.

The nucleated unicellular organisms, including most of the protozoa and algx, also multiply for the most part by simple division, but a new phenomenon makes its appearance here, as we 
shall see presently. In the process of the division of nucleated organisms, the nucleus plays an important part, undergoing a series of complex changes by which it finally becomes two nuclei similar to the original, one in each of the new cells (E., p. 226).

We see thus how it is possible to speak of the "immortality" of such organisms, since the substance of the parent does not degenerate, but is divided up after increase in dimensions.

This mere increase in size does not, of course, involve the acquirement of any new capacities of adaptation to the surroundings. The new material added is built up like that from which it grows. Accordingly, we find, even in such unicellular organisms as the protozoa and algæ, that a further process has been developed, which is the gradual beginning of the wonderful phenomena of sex, that play so large a part in the beauty of the world and the brave deeds done in it.

We find, to begin with, that two similar individuals fuse together, forming one single larger individual, which may then proceed to divide in the old way, or it may split up into a number of smaller individuals, each of which grows up to the normal size, and may then continue to multiply in the simple way of division into two. In either case the result is the union in the new generation of the qualities of two individuals, which, however similar they may appear to the eye, will almost invariably differ in their modes of reaction to surrounding changes, as also in their capacity for "variation," as we shall see later. This simple process of union of two individuals, in which we cannot as yet speak of either of the pair as being male or female, is known as "conjugation."

It was supposed for some time that a race of unicellular organisms, continuing to multiply merely by subdivision, sooner or later died out, unless conjugation occurred. The race was thought to undergo senile degeneration, and to be rejuvenated by conjugation. But it is not clear how this was to be brought about, otherwise than by the combination of the capacities of two different individuals, and it has now been shown that, if a proper supply of the materials necessary for growth is provided, there is no need for conjugation, and the vigour of the race remains unimpaired.

It will be clear that, since the advantage to be derived is the combination in the new individual of different qualities, there is not a great possibility of variety of experience in conjugation between cells of similar situation and habit, especially when the organism is not an actively motile one. The opportunity for conjugation occurs only between cells that chance to be in proximity to one another and have been exposed to closely similar conditions. The next step, therefore, and one taken very early, is the differentiation of the two gametes, as they are called, into two kinds of cells, one 
stationary and relatively large, the female; the other smaller and motile, the male. In the unicellular organisms, the whole organism is frequently converted into one female gamete, or into a number of male gametes. The latter swim freely, and one of them conjugates with a female gamete. But in the multicellular animals and plants, special organs are formed for the purpose of producing the two kinds of gametes. The female gametes are now called ova or egg-cells; while the male gametes have different names, spermatozoa in animals, antherozooids or pollen grains in plants (E., p. 226). The process corresponding to the conjugation of the simple organisms is now called "fertilisation." The organism itself as a whole becomes modified, the ova-bearing or female organisms being different in many ways from the male; in some cases extraordinarily so. The organ in which the ova are formed is known as the "ovary"; that in which spermatozoa are formed is the testis.

The material from which the sexual cells are formed in the course of the development of the young organism from the fertilised ovum is very early separated from that which becomes differentiated into the various organs of the body. It thus retains the whole of the characters of the gametes from which it has resulted, and, even in the adult, is but little affected by changes in the rest of the organisms. In a certain way we may speak of the "continuity of the germ plasm" from generation to generation. The question as to how far it can be influenced by changes affecting other parts of the organism will be discussed later.

When two similar cells conjugate there is, along with the general admixture of cell substance, a dissolution of the nuclei, followed by formation again of a single mixed nucleus. In the process of formation of the male and female gametes in the true sexual process, on the other hand, half the nuclear material is thrown off, so that the final fertilised ovum contains the normal amount, half of its own, the other half derived from the male element.

Although the process of fertilisation described above is the normal one, the ovum until fertilised remaining stationary with no cell division or growth of the new organism occurring, it is remarkable that, in some exceptional cases, the unfertilised ovum is able to develop. In such a case as that of the bee, this so-called "parthenogenesis" is a normal fact, the fertilised cells becoming the worker bees, the unfertilised cells becoming the males, called "drones." In other cases, such as the sea-urchin, the ova, although in the normal state of affairs requiring fertilisation, can be stimulated to development by chemical or physical means of various kinds.

In order that the ovum may become fertilised, it is clear that the spermatozoa must obtain access in some way. When the eggs 
are laid before fertilisation, as in the frog or fish, the male is required merely to deposit a liquid containing spermatozoa over the mass of eggs. In birds, although the eggs are laid and development proceeds outside the parent organism, the presence of the hard shell necessitates the introduction of spermatozoa into the duct of the ovary, so that they may enter the ovum before the outer layers are deposited on it. For this purpose, the male brings the orifice of the tube down which the secretion of the testis is poured into contact with the orifice of the oviduct and ejects the spermatozoa into the oviduct. A similar process is, of course, necessary in the mammalia, and in special cases in the lower organisms, where the fertilised ovum remains within the mother's body and completes its development up to an advanced stage therein. Here the male is provided with a special organ, the penis, for the purpose of more effective introduction of the spermatozoa into the oviduct of the female. The act of doing this is associated with feelings of pleasure in both sexes, as is obviously necessary to ensure the continued existence of the race by the froduction of offspring. It may be noted that a process of the kind is often present although the fertilised eggs are afterwards laid and develop outside the body of the female, as in many invertebrates.

The large size of the female gamete, or egg, as compared with the male gamete, especially striking in the bird and reptile, is m inly due to the fact that it is provided with a store of food material for the growth of the young animal, but is also used by ourselves for food. In the mammal, the food material is supplied by diffusion from the blood of the mother to that of the growing embryo. An organ, in which ramifying networks of blood-vessels from both sources lie side by side, known as the placenta, is present for this purpose.

A word may be said here in regard to flowers. It is by no means always realised that their beauty is, directly or indirectly, connected with the process of sexual reproduction (E., p. 227). This fact that they are, as has been said, "naked and unashamed," and the absence of any suggestion of unseemliness should give us matter for thought. Although the male and female gametes, pollen grains, and ovules, are usually parts of the same flower, a variety of devices exist in order to favour cross-fertilisation. The pollen grains are not motile, but in some cases, as the grasses, they are produced in enormous quantity, and, being very light, are blown about by the wind. In other cases, the brilliant colouring, the scent, and so on, serve to attract insects of various kinds to suck the sugary solution, nectar, from the depths of the flower. In doing this, they brush against the stamens and carry away pollen from them. A visit to another flower deposits part of the pollen 
on the stigma, whence the pollen tube grows down through the tissue and fertilises the ovule.

There are many familiar differences, of no apparently serious importance, between the two sexes in the greater number of animals. These are the "secondary sexual characters," and may be illustrated by the hairs on the face in man, the mane of the lion, the horns in some breeds of sheep, the crest on the newt, and so on. It is found that they are due to the internal secretion of the sexglands, and indeed to that of a particular kind of cell, found both in the ovary and in the testis, although, of course, the nature of the secretion is not the same. These are known as the interstitial cells. They are quite independent of the germ plasm from which the ova and spermatozoa arise. The chemical substances responsible for the effects seem to be sometimes inhibitory, preventing the growth of hair on the face in women, for example; sometimes excitatory, as in the Herdwick ram, where removal of the testis in the young animal stops any further growth of the horns.

Attempts to assign the difference between the properties of maleness and femaleness to general physiological differences in metabolism cannot be said to have met with great success. Thus it has been suggested that the female is more prone to the synthetic or "anabolic" changes, the male to the "catabolic," or breaking down processes, supposed to be the bases, respectively, of inhibitory and excitatory phenomena (see P., pp. 42I-423). But the view that food material is made into a complex protoplasmic molecule, $\mathrm{b}$ zfore being oxidised to afford energy, has been practically given up as our knowledge of cell processes has grown. Indeed, the conception of the universal occurrence of anabolic and catabolic stages as parts of the same chemical reactions does not seem to hold. In its application to the two sexes, it is pointed out that the male is the more active and enterprising, the female slower and more conservative. But the reader will be able to call to mind many cases to the contrary, and the distinction, like many other supposed sexual ones, is probably not of this nature at all, but merely incidental.

Although the chick, when it is hatched from the egg, is able t) pick up its own food, it is a familiar fact that the young of mammals are comparatively helpless for some time after birth, so that they depend on being fed with milk, secreted by special glands possessed by the mother. These mammary glands, as they are called, have been developed in the course of evolution from glands in the skin. Milk itself contains all the constituents required by a complete diet, such as we learned in our second chapter - a sugar, lactose, fat in small globules, protein of two kinds, salts, and the necessary accessory factors. It should be noted, however, that 
cow's milk may not contain sufficient of the anti-scorbutic factor to suffice for the human infant, and may require the giving of fruit juice in addition. The mammary glands increase greatly in size during the time that the young animal is growing in the womb. The stimulus to their growth is provided by a chemical hormone, formed in a peculiar tissue which takes the place of the ovum after it has left the ovary and been fertilised. This structure is called the corpus luteum, from the yellow pigment contained in it (luteus= yellow). The nature of the stimulus which excites secretion of milk when the young animal is born is not yet clear. It has been supposed that an inhibitory hormone is produced either by the growing foetus itself or the placenta, so that when these have left the body of the mother, the mammary gland is freed from the agent which prevented its natural secretory process from being manifested.

Heredity.-It can scarcely fail to arouse astonishment that such minute structures as the ova and spermatozoa contain the potential capacity. of developing into organisms similar to the parent organisms, even to details. It is obvious that, although the ova of the cat and dog are so much alike, yet there must be represented in them, in some way or other, the characteristics of the particular animal. Various theories, resting on very insufficient evidence, have been put forward, but they need not detain us.

There are, however, two aspects of the question which require a brief consideration. The first of these concerns the facts of inheritance associated with the name of Mendel, who was abbot of a large monastery near Vienna in the middle of the nineteenth century. He found that certain inherited characters are subject to two laws. For the sake of illustration, let us take the case of the peculiar "waltzing" mice. If a normal mouse is crossed with a waltzer, the offspring appears to be normal, and it might be thought that the peculiar quality had been lost. But when these offspring are bred together, it is found that the quality reappears in some of their young. So that it was still present, but had been overpowered by the normal quality in the first generation. Hence this quality is called "dominant," while that of waltzing is "recessive." The further remarkable fact is that there is a particular proportion between the number of individuals of the normal kind and that of the waltzers, namely, three of the former to one of the latter. How is this to be accounted for? Since the original pair may reasonably be regarded as contributing an equal number of the factors in question, we may take it that they are also so present in the total generation of three normal and one waltzer. What happens then is evidently that the factors must be arranged in the following way : one individual contains only dominant factors, another only reces- 
sive factors, while the other two contain equal amounts of both. But in these latter, the recessive factor is prevented from showing itself by the presence of the dominant factor. We have then some individuals which are pure-breed, so that if mated with similar ones, the progeny must be pure-breed. But those individuals which contain dominant factors only cannot be distinguished from the mixed ones, whereas those which show the recessive factor must be purely recessive and can be depended upon to breed true. This process is known as the segregation of pure gametes. The practical object of breeding new races is thus to obtain the desired quality as a recessive factor. Take the case of wheat. It is desired to obtain a variety resistant to the attacks of the fungus known as "rust." A race has been produced in which this character is recessive, and hence can be bred true.

It is not to be supposed that all inherited characters obey Mendelian laws, and there are many complications in detail which cannot be entered into here.

The second aspect of heredity which requires notice is the problem of the inheritance of characteristics acquired in the lifetime of the parents themselves. It has been held by some that the germ plasm conveys only those factors derived from distant ancestry, and that it is in no way affected by what happens to the individual organism which is its temporary host. There seems, indeed, to be no satisfactory evidence of any kind of mutilation happening to the parent being transmitted to the offspring, and the germ plasm, as far as we know, is independent of nervous connection with the rest of the organism. At the same time, it cannot be denied that it is accessible to chemical agents, and if such are produced by the various tissues, any alteration in them must have its effect. It will be clear that the removal of any part which leaves similar structures untouched cannot be expected to have any chemical effect of the kind mentioned, while the removal of any organ which is the only representative of its particular tissue is usually followed by death. Thus the loss of a leg leaves similar tissues intact, while that of the liver or suprarenals is fatal. That the germ plasm is accessible to chemical influence is shown by the experiments in which guinea-pigs were allowed to breathe the vapour of alcohol. The offspring of the alcoholized animals were deficient in strength and vitality, and often showed coarse abnormalities. The effects were transmitted through several generations, even more marked in the later ones, although no exposure to alcohol had been made since that of the original parents. No changes in the sexual glands were visible under the microscope, but the effects produced would be too subtle to be detected thus.

Variation.-Although the offspring are very like their parents, 
it is well known that there are differences of a more or less marked degree. These "variations" are often called "spontaneous," not as implying that they are self-produced by a so-called inherent tendency to vary, but that we cannot, as yet, assign an actual cause to them. In any case, they are made use of by natural selection for the production of new races and species. Darwin speaks of "The Origin of Species by means of natural selection, or the preservation of favoured races in the struggle for life," and he sums up the general theory as follows: "As many more individuals of each species are born than can possibly survive, and as, consequently, there is frequently recurring struggle for existence, it follows that any being, if it vary however slightly in any manner profitable to itself, under the complex and sometimes varying conditions of life, will have a better chance of surviving, and thus be naturally selected. From the strong principle of inheritance, any selected variety will tend to propagate its new and modified form." The last sentence may be also put thus: An individual which shows a new character is more likely in general to leave progeny possessing this character than are those without it. If it is one that enables its possessor to make better use of the forces of the environment, this individual is better situated, especially by its longer life, to leave more progeny, thus increasing the probability of the permanence of the new character.

Adaptation.-We see that, according to the view expressed in the last paragraph, the "adaptation" of organisms to their surroundings, or their fitness to their environment is indirect. On the other hand, certain writers have strongly advocated the existence of direct adaptation. No really conclusive proof of any case has yet been brought forward, and if we consider what it means, it must be admitted that it seems very unlikely. It would mean that the reaction of an organism to a new influence is such that the effect of this influence is to produce either a means of making appropriate use of it, or of meeting it, if injurious, by an appropriate defence. Since the reactions of an organism are necessarily conditioned by its structure and properties, it is difficult to see how a totally new condition would find the capacity of an appropriate reaction. The reaction may chance to be a favourable one, but that is not direct adaptation. The only strong evidence is derived from two characteristics of bacteria. The first is, that in certain cases bacteria grown on a medium, which they are unable to utilise as food, gradually develop in successive generations the power to do so. The weak point here is that we are dealing with several generations, and we cannot be sure that in the first culture there might not be a few individuals capable of utilising to a small extent the new food material. If so, these would 
multiply and the capacity be increased by natural selection in the ordinary way. To be convincing, the development of the power must be brought about in a single individual.

The second evidence is from the production of "anti-bodies" to the action of bacteria (see p. I4I above). Before this can be accepted as direct adaptation, we need to know much more about the nature of the processes at work here. Unfortunately, hitherto, investigations have chicfly resulted in the invention of names for phenomena without explaining them.

Struggle for Existence.-Some incorrect and mischievous interpretations have been made of the meaning of this phrase. It has been taken to imply a conscious perpetual warfare between individuals of the same species. In point of fact, those races are most abundant which rely on social co-operation to make use of the forces of Nature. Darwin himself points out that he uses the "term in a large and metaphorical sense, including dependence of one being on another."

The reader may remember that German writers defended the bringing about of the late war, and the horrible way in which their army carried it on, as justified by the "struggle for existence," being a necessity for the progress of the human race. But when anti-social methods are made use of by a nation acting as a wild beast, it becomes necessary for the more civilised nations to destroy that beast or render it incapable of doing further damage, notwithstanding the fact that much that is valuable may be lost in the process. Moreover, when a nation adopts the savage method of enforcing its systems and ideas by aggressive warfare, it becomes the duty of the civilised nations to oppose it by the use of what might appear to be the equally irrational method of defensive warfare. But there is no alternative, and the onus lies on those who started the process.

Akin to the view of those who hold to the importance of mutual aid as the chief factor in progress is that of Claude Bernard, who points out that organisms develop by adaptation to cosmic conditions, not by struggling against them. The living being is not in contradiction to external forces, but is a part of the total life of the universe. Bacon, again, said that "Nature is to be commanded only by obeying her."

To avoid misconception, it should be pointed out that such views as the above do not in any way lessen the urgent necessity for effort in discovering new means of making use of the forces of the outer world, and of the most effective ways in which social co-operation makes advance possible, not only in science and industry, but in the arts that make life beautiful. 

PART II 


\section{LABORATORY WORK}

NOTE.--The experiments described below will be found to vary greatly in their difficulty and in the cost of the necessary apparatus. But I have not thought it desirable to omit fundamental ones on either of these grounds, since their performance is possible in many existing laboratories, and should become so in the future to a much wider extent elsewhere.

The student may require the actual co-operation of the teacher in some instances. Certain of these may become demonstrations. It is desirable, however, that the student should, if possible, repeat the experiments for himself. This would naturally depend on the time available.

If the course is held in a completely equipped laboratory, the teacher may well be able to supplement the exercises given here.

\section{CHAPTER I}

The Microscope.-Since this instrument will be very frequently in use, it is well to understand, in their main outlines, the principles on which it is constructed. A few experiments with lenses will also give information needed for the study of the eye at a later time.

Take a biconvex lens of short focus, say about an inch, and note that a large image of an object placed close to it can be projected on to the wall of the room. The object and the image are at conjugate foci, and there will be found to be numerous pairs of such positions. The size of either is in proportion to its distance from the lens.

An appropriate object is a small black cross on a piece of ground glass. The glass is illuminated by a bright light behind it.

For many purposes, a small hand-feed arc-lamp is almost a necessity. The best type is that in which the carbons are at right angles to each other, since the bright crater in the positive carbon is not obscured by the negative. The addition of a condensing lens enables parallel rays to be obtained, and also the illumination of a small area with intensity.

The lens of short focus used in the experiment above represents the objective of the microscope, at that end of the tube nearest the object examined. But the great distance of the large real image makes this an impossible method of obtaining the magnification desired. If, however, we place a second convex lens in the course 
of the rays at a few inches distance from the first, we bring the rays to a focus at a short distance away. . A real image is formed here, as may be seen by receiving it on ground glass. This lens corresponds to the field lens of the ocular of the microscope, and this lens may be used for the experiment. The eye-lens has the function of magnifying the above image, just as an ordinary pocketlens does. In this case the image is not real, the eye being placed close to the lens in the emergent beam. The image on the retina in the eye is produced by the refractive system of the eye itself.

A graduated scale for measuring objects, or ruled in squares for counting their number, may be placed at the position of the fucus of the field lens, and thus be seen superposed on the object.

Another form of ocular is sometimes used. In this, no real image is formed: the eye-lens being closer to the field lens than the position of the focus of the latter.

In making experiments on the properties of lenses, they may be held in retort stand clamps.

The other details of the microscope stand can best be studied on the instrument itself. Below the stage on which the object is placed, we have arrangements for sending light through this. The tube containing the observing lenses is provided with means for accurately and conveniently bringing it to the correct distance from the object in order that the image may be formed in the ocular at the right place. Various objectives and oculars of different focal distance or magnifying power can be placed in position in the tube.

An appropriate microscope is that sold for bacteriological purposes, and may be obtained from several British makers. It should possess a sub-stage condenser for illumination with high power objectives, and also a dark-ground condenser to fit into the same tube as the ordinary one. Objectives of $25 \mathrm{~mm}$. and $4 \mathrm{~mm}$. focus are required, and a $2 \mathrm{~mm}$. oil immersion is desirable. One of the oculars should have a micrometer scale.

Nature of Protoplasm.-One of the protozoa, Amocba, is the best object for this study. As Professor Graham Kerr points out in Nature (3 Ist October I9I8, p. I66), the species required is the large form, which is to be found in water containing abundant food material and oxygen. Such a situation is the water trickling from a boggy spot.

If masses of weeds be collected from a pond and allowed to putrefy in water in a number of shallow covered dishes, amœbx can often be found in the slime scraped from the surface of the plants in some of the dishes. They are said to be most abundant in about a fortnight, and then commence to disappear.

Another way is to collect the upper layer of the ooze from the bottom of a shallow ditch or pond and allow it to settle in tall 
narrow jars. The amoebæ collect at the surface of the ooze as it deposits, and the layer may be removed with a pipette and the process repeated in a series of test-tubes. If not used at once, some green algæ, such as spirogyra, should be added to give oxygen.

They can often be obtained from dealers.

Should a source of this Amoba proteus not be at hand, a culture may be made from garden soil by the method described by Goodey (Nature, 25th July I918). Although most of those obtained are of the smaller kinds, large ones may sometimes be found. The following somewhat simplified method will serve our purpose. Boil some hay or grass in water. Filter. Neutralise. Place a layer 2 to $3 \mathrm{~mm}$. deep in several Petri dishes. Add to each a gram or so of garden earth. Keep in the light. After two or three days, according to the temperature, amœbæ may be obtained by floating a cover-glass on the surface for a minute or two in order to allow the organisms to fix themselves. Rinse gently with water, and a cleaner preparation will be made than if material is removed in a pipette. It is well to place a short bit of hair on the slide in order to avoid pressure on the organisms. If the surface film is used, a small quantity of the liquid is to be placed on the slide before inverting the cover-glass into position. Numbers of bacteria and ciliate protozoa will be seen, especially if the material from the bottom be taken. If the culture becomes nearly dry, encysted forms of amœbæ will be found. The larger ones may be picked out and transferred to fresh culture medium.

In addition to observing the properties of the protoplasm and the formation of pseudopodia, the nucleus, the contractile vacuole and the food vacuoles should be noted, especially the spherical form of the latter. (See Huxley and Martin's "Practical Biology," pp. 2I, 22.)

For electrical stimulation two strips of tin foil, or better, thin platinum, are cemented on to a microscope slide with Prout's glue, leaving a space of $2 \mathrm{~mm}$. between them in the centre. Each of these is connected to one of the terminals of the secondary coil of an induction apparatus, as described below (p. 205). by means of a fine wire which may be held in contact with the foil by a little lead weight. A weak stimulus must be uscd to begin with, and gradually increased until an effect is produced. A strong stimulus kills the organisms at once; their contents pass into the water, and are dispersed therein. Note the significance of this fact as regards the liquid nature of the protoplasm and the necessity for an outer membrane of some kind.

The Leucocytes of the Blood.-If amøeba are not to be obtained, many of the facts of the preceding section can be made out on the colourless corpuscles of the blood. A high magnifica- 
tion is necessary. A drop of blood is obtained by pricking the finger or from the heart of a frog which has been killed by chloroform. The cover-glass should have vaseline painted around its edges for a millimetre or so on the lower surface before placing it on the blood. This is to prevent drying of the preparation. The leucocytes will be found in the spaces between the columns of red corpuscles. Movements will be slow or absent in the case of the human blood unless the slide is warmed to body temperature. This can be done by placing the slide on a piece of sheet copper which has a hole to admit the light from below. This piece of copper has a long narrow part projecting a few inches beyond the edge of the stage. It is warmed by means of a small gas burner, the temperature not being allowed to rise at the place where the slide rests on it more than just feels warm to the finger.

Movement of Protoplasm.-This should be observed in the hairs found on the stamens of Tradescantia. . The species, $T$. virginica, with violet flowers, is grown in most gardens. The cell sap, being coloured, obscures the protoplasm somewhat, but it can easily be seen in the form of a layer lining the cell wall and filaments stretching across the cell. There are continual streaming movements, and the filaments change their position from time to time. Two or three hairs are picked off with forceps by seizing them at their bases. Mount in water.

There is a greenhouse species, $T$. discolor, with colourless flowers and variegated leaves. The cell sap being free from colour, the protoplasmic movements are more easily seen.

Dark-Ground Illumination.-The condenser used is sold in various forms by dealers. Water is placed between it and the bottom of the microscope slide to prevent the oblique rays being reflected. The method is of especial value in observing fine particles, with their Brownian movement and its cessation on stimulation of protoplasm. It is very useful in observations on bacteria. A brilliant source of light is required, and the small arc lamp will probably be found best, using a condensing lens to make the rays parallel. Large granules will appear too dazzling, but the minute ones need to be well lighted. In using the method with living cells, it is well to introduce a flat-sided glass vessel filled with water between the lamp and the microscope in order to cut off heat rays. It may also be advisable to add some quinine sulphate dissolved with the aid of dilute sulphuric acid to absorb ultra-violet rays.

Brownian Movement.-A cake of dry water-colour gamboge is rubbed in distilled water and a drop placed on a slide. The finer the particles the more vigorous the movements. They can be seen quite well with the ordinary form of illumination, but much 
better by the dark ground method. The object of using distilled water is to avoid the aggregating effect of the lime salts in tap water (see under "colloids" below).

To see the fixation of the particles by a gel, rub the gamboge in warm 5 per cent. gelatin, place a drop on a warm slide, cover and watch while setting takes place. The movement will gradually disappear.

Surface Tension.-Soap solution is made with pure sodium oleate. Make a 2.5 per cent. solution in distilled water in the cold. It may take a day or so to dissolve. Add one-third of its volume of strong glycerine, and after shaking, allow to stand for a week in a stoppered bottle in a dark place. Then remove the clear solution from underneath the scum by means of a siphon. Add a drop of strong ammonia to each 200 c.c. and keep in a stoppered bottle in a dark place. Do not return any that has been used to the stock bottle.

In the preparation of this and other solutions required later, a chemical balance weighing 50 or $100 \mathrm{gm}$. and sensitive to I $\mathrm{mg}$. will be required. It is convenient to replace the left-hand scale pan by a flat-bottomed porcelain basin. This is carefuly counterpoised by a piece of lead suspended on the hook at the top of the opposite scale suspension. A porcelain pan can be washed, so that it is unnecessary to use paper for the weighing of chemicals, which must never be put into the metal scale pan.

A useful tube for blowing bubbles is a wide glass tube, such as the chimney of certain paraffin lamps. A perforated cork is fitted in the narrow end, and a short piece of $\frac{1}{4}$-in. tubing inserted in the hole. Dipping the wider end into soap solution, a film is made closing the end. This is blown out into a spherical bubble and the end of the finger placed on the mouth-piece. The bubble remains the same size; but if the finger be withdrawn, surface tension causes the bubble to contract, driving air out.

Make a film on the wide end of a funnel, closing the end of the stem with the finger. As soon as the finger is removed, the film proceeds to rise up to the narrowest part of the funnel. Thus, surface energy is doing work in raising the weight of the film.

For the ring experiment iron wire serves well. A circle is made with a projecting handle. The end of that part forming the circle may be soldered to the base of the handle, or merely twisted around it. A little loop of fine sewing silk is tied to a point on the ring, so that the loop is suspended about the middle of the space. It saves trouble if it be tied to the opposite point of the ring also, but the suspensions must not pull tightly. Dipping the ring into soap solution in a flat dish, a film is formed in which the loop floats. By moving it about with a needle, it can be made to take any shape, 
as long as the film is present inside as well as outside the loop. If, now, the film inside the loop is broken by touching it with a pointed bit of filter paper, the tension outside pulls the loop into a circular shape.

This film on a ring may also be used to show that a needle can be dropped through without breaking the film. The needle should first be wetted with the soap solution.

To see the spherical form taken by drops of liquid requires some trouble and skill. The method recommended by Boys is to pour into a bottle a tablespoonful of olive oil together with a mixture of nine parts by volume of rectified spirit (or methylated spirit containing no petroleum oil) and seven parts of water. Shake up and leave until the oil has separated again. It may be either above or below the alcohol, according to the specific gravity of the latter. Fill a beaker with a similar mixture of alcohol and water. If the oil had risen to the top in the bottle, add a little water to the liquid in the beaker by a pipette dipping about half-way down. Take some of the oil in a pipette and empty into the middle of the keaker. If it sinks, a little more water is wanted in the lower half $\mathrm{cf}$ the beaker; if it floats, a little more alcohol is wanted in the upper half. To see the shape truly, it must be looked at from above the liquid, since the curvature of the sides of the beaker distorts the image. When the right specific gravity of the alcohol has been obtained, more oil may be added to the drop, best from a tap-funnel slowly.

Adsorption.-A bubble is blown as above, but using a dilute solution of saponin in distilled water (about 0.5 to I per cent). Allowing the bubble to contract, or by sucking air out of it, it becomes more or less rigid and goes into folds (Ramsden). Solid particles of saponin will be seen in the film, and especially' in the drop at the lower end of the bubble.

Add charcoal in powder to a dilute solution of crystal violet until the colour is removed. Filter off the charcoal and wash it on the paper with acetone. After the water is replaced by acetone the drops falling through will be stained violet. It is better to make the acetone slightly acid with a drop of hydrochloric acid, on account of the fact that it is the free base of the dye that is adsorbed.

Cell Membrane and Permeability.-Having found an amœba or leucocyte, which is known to be living by its movements, add a dilute solution of aniline blue by placing a drop at one end of the cover-slip and a fragment of filter paper at the opposite end. The organism does not stain as long as it is alive, although any bits of dead material become blue.

Slices of red beet are washed under the tap in order to remove the contents of the cells injured by the knife. Left in tap water, the 
slices do not give up their pigment. If boiled, so as to kill the cells, the water becomes red.

To show that the living cells are also impermeable to sugar a chemical test must be used. Owing to its aldehyde group, glucose reduces copper salts in alkaline solution. The sugar in beet is, however, cane sugar, which has no free aldehyde group. It must be hydrolysed by boiling with acid. The test may be made with the water in which living beet has lain, and also with that in which it has been boiled. Add to a sample in a test-tube a drop or two of strong hydrochloric acid, boil for a minute, or immerse the end of the test-tube in boiling water for a few minutes. Cool. Add a few drops of copper sulphate solution, a crystal of Rochelle salt, and then sufficient sodium hydroxide to make a deep blue solution. On boiling again, a red precipitate of cuprous oxide is formed if a reducing sugar is present.

The effect of other methods of killing the cells of the red beet may be tested by adding chloroform, formaldehyde, or acid. Substances which lower surface energy, such as saponin, bile salts, or amyl alcohol, also have the effect of allowing the pigment to escape. In some specimens, it may be possible to observe the reversible increase of permeability produced by sodium chloride. Take a I per cent. solution in distilled water, change several times, and the pigment may be seen to escape slowly from the cells. The normal state is reproduced in such cases by the addition of a calcium salt in small amount, most simply by changing to hard tap water.

Dilute acid and alkali may also be tested, say, o.o I per cent. hydrochloric acid and a similar strength of sodium hydroxide.

\section{Osmotic Pressure}

Blood Corpuscles.-Make a preparation of blood from the finger as described above (p. I66). Measure the diameter of a number of corpuscles and take the average. The measurement is done by a scale in the eyepiece, the value of its divisions having previously been determined by the aid of a scale divided into $0.001 \mathrm{~mm}$. placed on the stage and observed with the same objective as that used for the blood preparation.

On allowing water to run under the cover-slip, the corpuscles rapidly swell up and their contents escape, leaving the framework as a nearly invisible disc. This action of water is, however, so vigorous that it is difficult to follow the series of changes. It is better to take a solution of sodium chloride of about 0.3 to 0.4 per cent.

Make next solutions of cane-sugar of 15, IO, and 5 per cent. 
Place a drop of each on a slide, and add a drop of blood, mixing with a needlc. The corpuscles in 10 per cent. solution $(=0.3$ molar) will be practically unaltered in size. Those in 5 per cent. will be increased, those in I 5 per cent. will be decreased. Since the osmotic pressure of the serum is not always exactly the same in different cases, some increase or decrease may be produced by IO per cent. cane-sugar. If the former, try a slightly stronger solution; if the latter, a slightly weaker solution.

Take 2 or 3 c.c. of 10 per cent. cane-sugar solution in a testtube. Add a drop of blood. The corpuscles will deposit gradually, leaving the solution colourless. Add water slowly, noting how much is added. At a certain dilution, the solution will begin to become coloured with hæmoglobin, owing to breaking up of the corpuscles (hemolysis). Note that the dilution required is greater than corresponds to the solution in which the corpuscles maintain their normal size (isotonic solution). This is due to the fact that they are able to swell to a notable degree before bursting.

Similar experiments may be made with $0.3,0.9$, and 2 per cent. sodium chloride. The isotonic solution will be found to be 0.9 per cent. Note that a 0.3 molar solution is 1.75 per cent. and that 0.9 per cent. is only O.I 54 molar. A I.75 per cent. solution makes the corpuscles shrink; it is liypertonic.

Next take a 0.3 molar solution of urea ( $=\mathrm{I} .8$ per cent.). It causes hæmolysis as if water. Dissolve the urea in 0.9 per cent. sodium chloride; no hæmolysis occurs. Hence the effect of the pure urea solution is not due to a toxic action of the urea, but to the permeability of the corpuscles for urea.

Saponin or ether, even in 0.9 per cent. sodium chloride, causes hæmolysis, which is due in these cases to a destruction of the osmotic properties of the cell membrane.

Take defibrinated blood, to be obtained from the slaughterhouse. Or better, cut off the head of a rat, collect the blood, and stir it with a feather. Put about ro c.c. into a graduated centrifuge tube, and centrifuge until the volume of corpuscles, read off on the scale, no longer changes its value. Pour off the serum, which is probably slightly coloured with hæmoglobin, so as not to disturb the deposit. This may be done by using a glass rod touching the lip of the tube. It does not matter if a little serum remains. Add I 5 per cent. cane-sugar, shake up the corpuscles with it gently and centrifuge again. The volume is less. Repeat with 5 per cent. solution, the volume is increased as compared with the original one.

For this experiment a simple hand centrifuge, as sold by the dealers, suffices. The hæmatocrite, in which smaller tubes are used and spun at a greater rate, is used for small quantities of blood, 
such as can be obtained from the finger. It is more expeditious, but rather more difficult The point at which no further change in volume occurs by centrifuging longer is recognised by the fact that the corpuscles are so closely packed together that the narrow column appears transparent red, instead of being opaque.

Plasmolysis.-Make a preparation of the hairs of Tradescantia virginica, the epidermis of the leaf of $T$. discolor, or a thin scction, made with a razor, of the root of the red beet. The protoplasm of the cells in each case is a bag containing coloured cell sap. If exposed to a solution which has a higher osmotic pressure than the cell contents, provided that the cell membrane is impermeable to the solute, water passes out and the protoplasmic sac shrinks, leaving gaps in places between itself and the cellulose envelope. Solutions of potassium nitrate may be used. As a rule, 0.1 5 molar (=I.5 per cent.) gives no effect, 0.25 molar (2.5 per cent.) has an obvious effect, 0.2 molar is about isotonic. This is cquivalent to an osmotic pressure of five atmospheres

Turgor.-Take the stalk of a flower, such as the daffodil or dandelion. It is stiff owing to the tension of the cell walls produced by the difference of osmotic pressure between the cell contents and the very dilute watery solution outside. As the tissue dies it becomes flaccid. The effect can be produced quickly by exposure to ether vapour in a test-tube, and, if the action has not been too great, recovery may be brought about by soaking in tap water.

The cclls may also be killed by putting a little water in the bottom of a test-tube, placing the stalk in the tube and then boiling the water.

- Contractile Vacuole.-To see the discharge of this vacuole in the amœba or other protozoon, add suspension of indian ink to the liquid in which the organism is. Do not use too much. When the vacuole contracts, the particles are driven away by the current from it.

Direct Measurements of Osmotic Pressure.-Such measurements are not easy, on account of the difficulty of preparing suitable membranes. To see the fact of the production of pressure, parchment paper may be used with a solution ( 5 to ro per cent.) of an electrolytically dissociated colloid, such as caseinogen or congo-red. The paper may be clamped in an osmometer of the pattern described by Moore and Roaf (Biochem. Journ., vol. 2, p. 34), or the simpler form of Roaf (Quart. Journ. Exper. Physiol., vol. 3, p. 79). It is possible also to fix the membrane on the edge of a small bell glass open at the top and provided with a flange at the lower edge. The membrane, in this case, must be glued on with gelatin, and parchment paper is not readily wetted with the solution. It should 
be first well soaked in warm gelatin solution, the edge of the glass being also immersed in the solution. After removal of both, the paper is left in position on the glass until the gelatin is set. It should then be hardened by immersion in 5 per cent. formalin. It is necessary to support it by a disc of nickel gauze, tied on by string or wire brought over the top of the glass. A narrow U-tube containing mercury is fixed in a rubber cork in the upper opening of the glass.

The caseinogen solution is made by taking the required amount, rubbing it in a mortar with a little water to which a drop of phenolphthalein has been added. Ammonia is dropped in until the colour has become pink. The volume is then made up in a measuring flask to the required value.

If possible, an accurate measurement should be made of the osmotic pressure of the colloids of blood serum, using as the outer liquid a 0.9 per cent. sodium chloride.

Exact determinations of freezing and boiling points are also rather difficult. The student should take that of distilled water by immersing a test-tube, containing a few c.c. of it, in a mixture of ice and salt; there should not be a large proportion of salt. A thermometer is immersed in the water, which must cover the bulb. Continually stirring by means of a ring of wire fixed in a glass tube by means of sealing-wax the temperature will fall steadily to $0^{\circ}$, and remain so for a little time. Taking out the tube from the freezing mixture, particles of ice will be seen floating in it, or a thin layer of ice may be present on the inside of the tube. The temperature will be seen to remain at zero until this ice has melted, provided that the stirring is continued. Repeat with a molar solution of sodium chloride $(5.85$ per cent.). The temperature will fall lower before remaining steady, and will remain at this lower temperature on removal until the ice has disappeared. Only a small amount of ice should be allowed to form, because it is pure ice (frozen water) that separates, leaving a more concentrated salt solution. The lowering of the freezing point by molar sodium chloride is about $3^{\circ}$.

In connection with the problem of electrolytic dissociation, it is well at this stage to compare the frcezing points of molar solutions of sodium chloride and of urea. We have already found that of the former. Make a molar solution of pure urea (=6 per cent.). It will be found to have a freezing point of $-1.86^{\circ}$ only.

A rough measurement of the boiling point of a solution may be made by the use of a flask in which it is kept boiling gently, while the bulb of the thermometer is suspended just above the level of the liquid. A bit of porous clay, such as the stem of a clay tobacco pipe, put in the liquid, will assist steady gentle boiling. 
The effect of a solute in lowering the vapour pressure of the solvent may be tested by allowing two vessels, such as flat porcelain capsules, one containing water, the other Io per cent. sodium chloride, to remain for some time side by side under a small bell glass, closed by resting the rim, greased with vaseline, on a piece of plate glass. The two vessels are weighed to begin with, and again after a few days. Water will be found to have passed from the vessel containing it in the pure form to that containing the solution.

Electrolytic Dissociation.-To make measurements of electrolytic conductivity with accuracy requires somewhat complicated

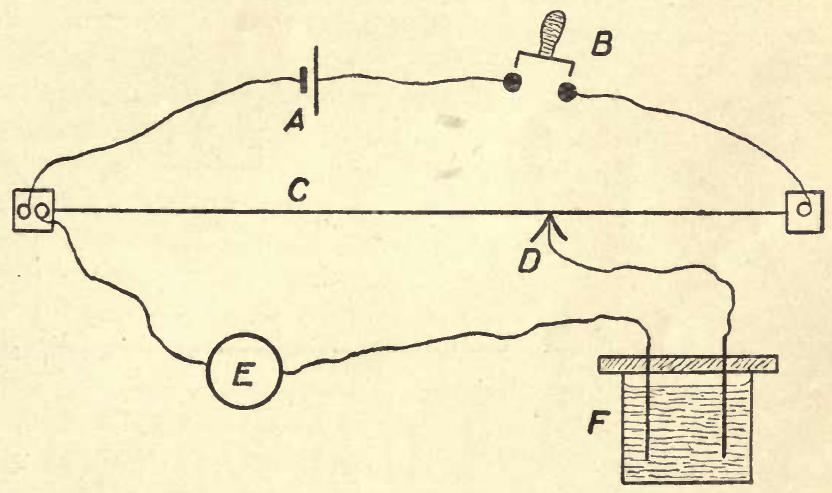

FIG. IO.

A, dry cell or storage cell.

B, key.

C, high resistance slide wire to obtain a fraction of the E. M. F. of the battery. For the present purpose, it need not be graduated, but this may be necessary for other work.

D, sliding contact.

E, galvanometer.

F, small beaker with the platinum electrodes.

apparatus. For our purpose it will suffice to use a galvanometer, and to observe the deflections produced by the same potential difference through the various solutions to be compared. The single-pivot galvanometer (one scale division =one micro-ampere), made by Paul, will be found to be convenient. The current is sent through the solution by means of two platinum plates, about $\mathrm{I} \mathrm{cm}$. square, immersed therein. 'They should have platinum wires welded to them, and the wires then fused into glass tubes passing through holes in a flat rod of ebonite, about $2 \mathrm{~cm}$. apart. The circuit is arranged as in the diagram (Fig. 10). The wires to the electrodes should be fine copper wires and inserted into mercury in the glass tubes, in order to make contact with the platinum plates. 
Take first normal hydrochloric acid, which can be bought. Adjust the slider until on closing the key a deflection is obtained which does not exceed the limits of the scale of the galvanometer. Note the maximum reading. This rapidly diminishes if the circuit is kept closed, on account of polarisation of the electrodes. In accurate work an alternating current is used to avoid this effect, as described below, and the electrodes are coated with platinum black (see Findlay's “Practical PhysicalChemistry," p. I 50).

Repeat the experiment, using acetic acid in the same molecular concentration. It is made by taking about 54 c.c. of glacial

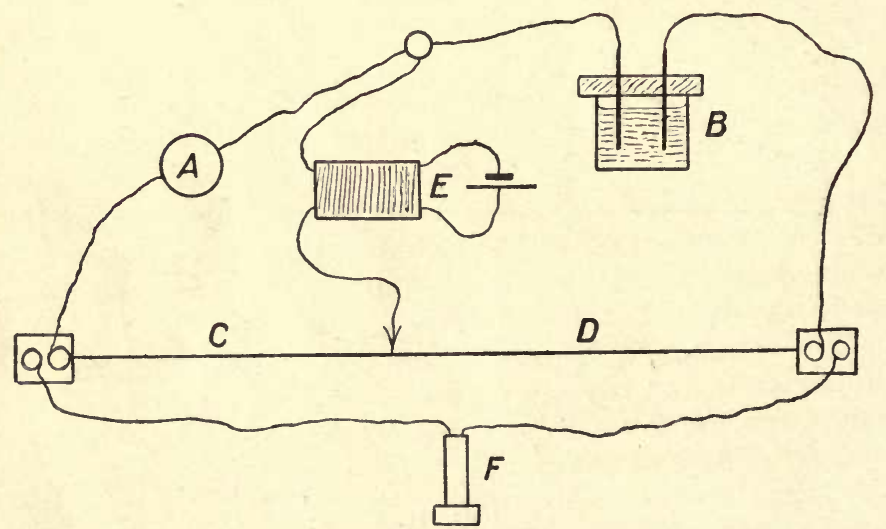

FIG. II.

A is a resistance of 1000 ohms.

$\mathrm{B}$ is the cell containing the solution to be measured.

$\mathrm{C}$ and $\mathrm{D}$ are the slide wire of one metre length, graduated.

$\mathrm{E}$ is a small induction coil, such as used for medical purposes, but provided with a

light steel spring as vibrating contact to give a high pitched note. The secondary winding is connected to the circuit.

$\mathrm{F}$ is a telephone.

acetic acid and diluting to a litre with distilled water. It is then titrated with standard sodium hydroxide, using phenol-phthalein as indicator. It is best to make it rather too strong and then diluting to the proper volume. The standard alkali can be bought, but should be tested against the normal hydrochloric acid. The deflection obtained with the acetic acid will be less than that with hydrochloric acid. Hence there are more conducting constituents in the latter than in the acetic acid. In other words, the strong acid is more highly dissociated electrolytically than the weaker acid.

To make more accurate determinations, the use of alternating currents is necessary. The following arrangement of the Wheat- 
stone bridge is used (Fig. I I). When C and D are of such relative lengths that no sound is heard, the resistances in the arms are such that $A$ is to $B$ as $C$ is to $D$, or $\frac{A}{B}=\frac{C}{D}$ i.e., if $C$ is in centimeters, and $A=1000$ ohms,$\frac{1000}{B}=\frac{C}{100-C}$ or $B=1000\left(\frac{100-C}{C}\right)$ in ohms.

If this apparatus is available, the resistances of molar and decimolar solutions of both hydrochloric and acetic acids should be compared. If dilution had no effect on the acid, the resistance of the deci-molar acid should be ten times that of the molar. In the case of the strong acid, this will be found to be very nearly what is found. In that of the weak acid, the resistance of the deci-molar solution will not be so much as ten times that of the molar acid, showing that dilution has caused the formation of a greater proportion of conducting ions than were present in a portion of the original solution containing the same quantity of acid.

Take next defibrinated blood and obtain some serum by the use of the centrifuge. Place the serum in the vessel used for the acids and determine its resistance, best by the telephone method, but it may be possible with care to see the effects when the first simple method is used. Replace the serum by an equal volume of the corpuscular deposit. The resistance will be much greater. That the lower conductivity of the corpuscles is not due to the absence of electrolytes from them, but to the fact that they are impermeable to these electrolytes and cannot therefore conduct an electrical current, can be shown by adding a small quantity of saponin. This, as can be tested, has itself a negligible power of conduction. But the conductivity of the blood corpuscles is greatly increased, because saponin destroys the cell membrane.

Indicators. - That it is the hydrogen-ion concentration of a solution to which these substances react can be made obvious by taking a solution of crystal-violet of about O. I per cent., and adding a drop to each of a series of dilutions of hydrochloric acid, beginning with twice molar and going down to one-thousandth molar. The dye is yellow in the strongest, green in molar acid, greenishblue in $0.1 \mathrm{~m}$., blue in $0.01 \mathrm{~m}$., violet in $0.00 \mathrm{I} \mathrm{m}$.

That different indicators react to different hydrogen-ion concentrations can be seen by taking a known volume of deci-normal sodium hydroxide (adding phenol-phthalein, a few drops of a O.I per cent. solution in alcohol), and adding from a burette decinormal phosphoric acid until the colour disappears. Note the amount of acid required. This indicator shows a concentration of hydrogen-ions of $0.0000000 \mathrm{I}\left(\mathrm{IO}^{-8}\right)$ normal as being acid. Add next to the same solution a few drops of o.I per cent. methylorange. The colour is yellow, that of the dye in alkaline solution. 
Add acid until it becomes red, and note that the extra quantity required is distinct. This indicator does not show a solution to be acid until its hydrogen-ion concentration has risen to $0.0 \mathrm{I}\left(\mathrm{IO}^{-2}\right)$ normal. The colour is orange-red at o.00I normal. The difference in the amount of acid required will, of course, be more obvious if 0.OI molar acid is taken instead of the O.I molar.

As exercises in the use of indicators, determine the hydrogenion concentration of urine, which will usually be found to be acid to methyl-red, just alkaline to methyl-orange. The colour of the latter dye in it is brownish, not yellow, corresponding to a hydrogen-ion concentration of $\mathrm{IO}^{-4}$ (see P., p. I89). Blood serum after exposure to the air is yellow to neutral-red $\left(=10^{-9}\right)$. Brought into equilibrium with the last fraction of a deep expiration (=alveolar air, containing 4.5 per cent. of carbon dioxide) it becomes orange-red. That is, just on the alkaline side of neutrality. Neutral-red in pure distilled water is red, not crimson. The slighest trace of acid turns it crimson; of alkali, yellow. It is thus a valuable indicator in the region about the neutral point $\left(10^{-7} \mathrm{x}\right)$. The saturation of liquids with gas mixtures, such as alveolar air, is performed in vessels containing a large volume of the gas in proportion to the liquid. For the above purpose, a stoppered bottle of about roo c.c. capacity will serve. Two or three c.c. of the serum with a drop of o.I per cent. neutral-red are placed in the bottle and the expired air breathed into the bottle through a glass tube. The stopper is replaced quickly, and the bottle rotated so as to make a thin layer of the serum over the surface.

\section{The Colloidal State}

Colloidal Gold is readily prepared by Faraday's method as follows :-Take a solution of gold chloride in pure distilled water, containing about one part of the salt in 8,000 of water. Put it in a clean bottle or flask. Add a drop of a solution of phosphorus in carbon bisulphide. Shake and leave for a few hours. A beautiful clear red solution is obtained.

This solution is shown to contain solid particles in suspension by passing a bright beam of light through it (Faraday phenomenon). The beam from an arc-lamp, brought to a focus by a condenser, is appropriate. To avoid disturbing reflections at the surfaces of the glass, the vessel may be immersed in water in a large beaker. This fact was described by Faraday, and correctly interpreted as showing the presence of particles of metallic gold. Tyndall pointed out subsequently that if the track of the beam be looked at through a Nicol prism, it is found to be polarised, being extinguished in a particular position of the prism. This shows that the size of the particles is near that of the wave length of light. 
As another example of a suspensoid, skake up kaolin (china clay) with distilled water, and allow the coarser particles to settle. The finest particles will remain dispersed for some hours, and such a preparation serves well for experiments.

Emulsoids.-Prepare a solution of gelatin by allowing a sheet of the dry substance, as sold, to soak in water until softened, and then dissolving it in hot water. As the solution cools, it sets to the familiar jelly.

Another emulsoid which does not set to a jelly is white of egg. This has another property, that of becoming solid when heated, as well known. In this state its properties change to those of a suspensoid.

The increased swelling of gelatin in the presence of acid may be shown thus: Allow a sheet to soak in water. Cut out a number of discs with a cork borer of about $\mathrm{I} \mathrm{cm}$. in diameter. Place some in distilled water, others in deci-normal hydrochloric acid. After some hours measure the diameters of a few of each.

Surface Tension and Dispersion.--Soap has a powerful effect in lowering surface tension. Olive oil usually contains a small quantity of free oleic acid, and when alkali is added, this forms soap (see later, page 190). Olive oil shaken with water forms an emulsion, but the drops of oil quickly coalesce and rise to the surface. If a very small amount of sodium hydroxide be added and the mixture again shaken, the soap formed lowers the surface tension at the contact of the water and oil, so that the drops have little tendency to unite, and a nearly permanent emulsion is produced.

Electrical Charge.-The simplest method of determining the sign of the charge on colloids is to take a U-tube of about a centimetre in diameter. Fill with the solution, and place in it at the upper end of each limb a piece of platinum foil connected with a source of potential difference of some 200 volts, such as the direct current house lighting supply. One electrode will be positively charged; the other negatively. A lamp should be inserted in the circuit to diminish risk, should the electrodes be accidentally brought into contact. The sign of the poles is determined by placing the two electrodes 2 or $3 \mathrm{~cm}$. apart on a piece of filter paper wetted with a solution of sodium sulphate to which phenol-phthalein has been added ("pole-finding paper"). The negative pole produces a red stain, owing to the alkali formed. After the connection to the tube has been made for some minutes, the space around and below one of the poles will become clear, orving to the repulsion by the electrode of particles of the same sign as itself.

Two typical suspensoid colloids are arsenious sulphide and ferric hydroxide. The former is negative; the latter, positive. 
Arsenious sulphide is made by passing a current of hydrogen sulphide through a saturated solution of arsenious acid in distilled - water and allowing the coarse particles to deposit. It may, with advantage, be dialysed to remove the dissolved gas. The process is described below.

Ferric hydroxide is made by taking a strong solution of ferric chloride (or better, ferric acetate, as used by Graham, on account of the greater hydrolytic dissociation of the salt of the weak acid). Place in a dialyser made by tying a piece of wet parchment paper over the wide end of a bell glass, such as used for osmotic experiments previously, but larger. Repeated changes of distilled water on the outside remove most of the hydrochloric or acetic acid formed by the hydrolysis of the salt. Colloidal ferric hydroxide may also be made by dissolving precipitated ferric hydroxide in ferric chloride and then dialysing to remove excess of electrolytes. The "solution of dialysed iron" of the "Pharmacopœia" may serve also, but it is better to prepare it.

Action of Electrolytes.-Make solutions of :

Potassium sulphate - o.I molar in $\mathrm{K} \cdot(0.88$ per cent.).

Calcium sulphate -0.01 molar in $\mathrm{Ca} \cdot$ (0.173 per cent. of gypsum).

Lanthanum sulphate -0.001 molar in $\mathrm{La} \cdots(0.0364$ per cent. of the cryst. salt).

Add equal volumes of each to three samples of arsenious sulphide. The precipitating power is about the same. Potassium sulphate in 0.00 I molar solution has no effect. Since the lanthanum solution is much more effective than the potassium, although the $\mathrm{SO}_{4}$ " ion is in only $\frac{1}{35}$ th the concentration, it is clear that it is the cation (positive) that is the active one, and the greater power of the bivalent and trivalent ions is obvious. Thus, a negative colloid is precipitated by a positive ion.

A similar series of experiments may be made by precipitating ferric hydroxide with the following solutions :-

Sodium chloride - O. I molar in $\mathrm{Cl}^{\prime}$ (0.585 per cent.).

Sodium sulphate - o.or molar in $\mathrm{SO}_{4}^{\prime \prime}(0.33$ per cent. of the cryst. salt).

Sodium phosphate-0.001 molar in $\mathrm{PO}_{4}^{\prime \prime \prime}$ (0.01 38 per cent of the acid phosphate).

The phosphate solution is made neutral to neutral red by adding sodium hydroxide. These three solutions will be about equal in precipitating power. Thus the electro-positive colloid is aggregated by anions.

The sign of the charge on kaolin in suspension may be determined by testing with the two series of salts. It will be found to be electro-negative. 


\section{Mutual Precipitation of Oppositely Charged Colloids}

Take a series of test-tubes containing equal quantities of arsenious sulphide, add gradually increasing quantities of ferric hydroxide. At a certain relative proportion, dependent on the concentration of the colloids, there will be complete precipitation of both. At other proportions, a compound colloid will be produced with excess of one or the other sign, and will remain more or less completely in suspension. As a rule, the ferric hydroxide solutions used will be found to be more concentrated than the arsenious sulphide.

Take also a 5 per cent. solution of egg-white and filter it. Make a part acid to neutral red by the addition of acetic acid, and another part alkaline with sodium hydroxide. The former will be precipitated by arsenious sulphide, not by ferric hydroxide; the latter, the converse. Thus we have produced an electropositive colloid by excess of $\mathrm{H}$-ions, and an electro-negative one by excess of $\mathrm{OH}$-ions.

Excess of Electrolyte. - If we add a solution of a precipitating ion in excess, it may happen that, instead of obtaining precipitation, the particles have conferred upon them a charge of the sign opposite to their original one, so that the concentration of the requisite precipitating ion is insufficient. They remain, in such a case, suspended. The experiment is rather difficult, on account of the fact that the actual amount required can only be found by trials. It varies with the dimensions of the particles. It may be tried with a suspension of gamboge obtained by pouring a small amount of an alcoholic solution of the gum-resin into a large amount of distilled water. Adding 0.0016 molar cerium chloride to an equal volume will precipitate it, whereas 0.I6 molar will probably not do so, or not so rapidly. If no difference is found, try intermediate concentrations. The distinction is seen best by shaking again after the first deposition and noting the second effect.

Staining and Electrical Adsorption.-Take some circles of filter paper of 9 or $10 \mathrm{~cm}$. in diameter. For good results, the paper should be the purest analytical preparation, and the dyes should be free from mineral salts. Congo-red may be dialysed, since it usually contains sodium sulphate and chloride. Immerse a circle of paper in a weak solution of crystal-violet and another in a weak solution of congo-red. The former rapidly takes on a deep colour, the latter very little. Add 0.5 per cent. sodium chloride to each of two fresh samples. The staining will be much increased in the case of congo-red, decreased in that of crystal-violet.

Rate of Chemical Reaction between Colloids. - The best way to observe the comparative slowness of this is to prepare a colloidal 
solution of the free acid of congo-red and add to it a suspension of aluminium hydroxide, which has been well washed. Metallic zinc in powder (zinc dust) may be used, but the experiment is not so striking. The colour of the mixture is at first that of the free acid, deep blue. Allowing it to stand, chemical combination slowly occurs, with the production of the aluminium or zinc salt. Combination can be hastened by warming. The solution of the acid is made by adding hydrochloric acid to a solution of congo-red. The deposit is suspended in water, and dialysed until free from acid. Contrast the rate of this chemical reaction in heterogeneous systems with the immediate deposition of saponin in the bubble made in the experiment on p. 168. 


\section{CHAPTER II}

\section{Chemical Composition of Organisms}

Carbon.-Heat a little yeast or any organic tissue in a dry testtube. It will char, owing to the production of carbon.

Hydrogen.-If the tissue had been previously dried in the above experiment, the deposition of water on the upper part of the testtube will show the presence of hydrogen.

Oxygen.-That the oxygen contained in the water of the above experiment comes from the tissue, and not from the air, can be shown by performing the experiment in an atmosphere of coal gas. The test-tube is closed by a cork through which pass two glass tubes, one to the lower end, the other ending just below the cork. The former is connected to the gas supply by means of a rubber tube, the latter to a Bunsen burner. Gas is passed through the tube until the air is displaced. The Bunsen burner is then lit and the tube heated as before.

Nitrogen.-Mix the yeast, dried, with some dry soda-lime. Heat in the test-tube. Ammonia is given off, detected by its smell, and its turning moist red litmus paper blue. The fumes given off when hydrochloric acid on a glass rod is held at the mouth of the test-tube are also characteristic of ammonia.

Sulphur and Phosphorus.-Boil with a little strong nitric acid to oxidise the sulphur to sulphate and the phosphorus to phosphate. Dilute with water. Filter if necessary. Test a part with barium chloride, precipitate shows sulphate. Add to another part a few drops of a strong solution of ammonium molybdate and heat. A yellow precipitate indicates phosphate.

\section{The Polarimeter}

A simple form of this instrument will serve to ascertain the fact of optical activity. In fact, if a strong solution of sugar or of egg-white be used in a vessel with flat glass sides, two Nicol prisms may suffice. A beam of light is sent through one of these, clamped in a retort stand, in order to polarise it. The beam then passes through the solution and, lastly, through the second Nicol, 
which must be capable of rotation. In the absence of the solution, adjust the position of the second prism so that the field is as dark as possible. Interpose the solution. If optically active, the field will become light. In the case of cane-sugar, the second prism (analyser) must be rotated in the direction of the movement of the hands of the clock in order to restore darkness; in that of eggalbumin, in the opposite direction. The actual amount of rotation must not exceed $\mathrm{I} 80^{\circ}$ in this experiment, otherwise confusion may result. If in doubt, dilute the solution until the fact of rotation can be only just made out.

A more accurate and sensitive polarimeter is necessary for certain important experiments with enzymes to be described later.

\section{Waste Products}

To prove the production of carbon dioxide in the course of vital reactions, all that is needed is to breath out through a tube

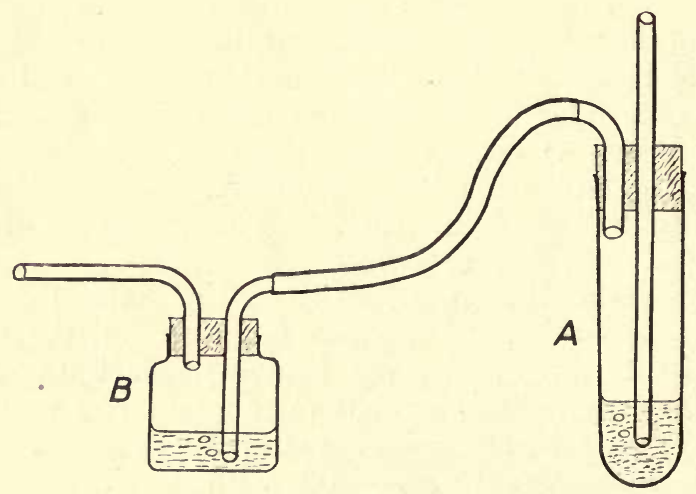

FIG. 12.-Urea Apparatus.

A, large test-tube, immersed in warm water.

$\mathrm{B}$, bottle containing acid, connected to a filter pump.

into recently filtered lime water, or solution of barium hydroxide. The precipitate is shown to be carbonate by its solution in dilute acetic acid.

The chief waste-product of nitrogen metabolism is urea, which is excreted in the urine. To prove its presence, the best method is to convert it into ammonium carbonate by the agency of the enzyme urease, found in Soy beans and elsewhere. A few beans are ground in a coffee mill, the powder sifted through a sieve, and a portion of what passes through is added to some fresh urine in a closed bottle. Allow to stand for an hour or two in a warm place. On opening 
the bottle, ammonia may be detected by its smell and the other tests described above. If not, add dry sodium carbonate, draw a current of air through the warmed mixture, and then through water made acid (blue) to congo red. This colour will turn red quickly, and more acid may be added from time to time. Fig. 12 shows the arrangement.

\section{Carbon Cycle}

Water Culture.-The solution to be used may consist of the following salts :

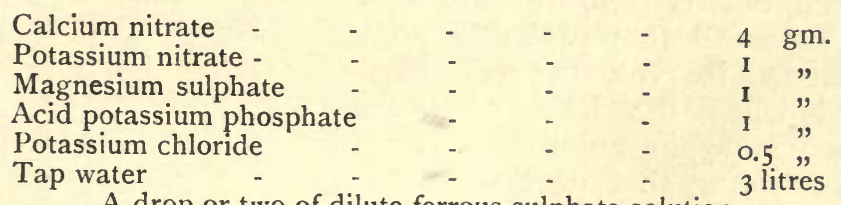

A drop or two of dilute ferrous sulphate solution

A large glass jar is provided with a wooden lid having a hole in the middle. The seedling of a Windsor bean, selected from a number which have been allowed to germinate between wet filter paper, is gently supported in the hole by means of bits of cork, so that the root dips into the solution. The wooden cover and the corks should have been soaked in melted paraffin wax. The weight of the bean, dried in air, is noted before germination. After the plant has grown to a foot or more in height, it is removed, allowed to dry in the air, and weighed again.

For success, growth must take place in a good light, the root being kept dark by a covering of brown paper on the jar. If exposed to the sun, the jar should be in a box filled with sawdust to prevent the solution becoming hot. The solution is changed at a few days' interval, and air blown through it occasionally to supply oxygen to the roots.

The experiment, of course, requires some weeks for completion.

\section{Action of the Green Plant on Carbon Dioxide}

Make first an analysis of atmospheric air. This may be done with the Hempel burette and two Hempel pipettes, one for caustic soda, the other for alkaline pyrogallol. For our purpose, it will be simpler to use a nitrometer tube, connected with a reservoir of mercury, and to perform the analysis in the tube itself. The arrangement is represented in Fig. 13. The tube A is graduated into O.I c.c. and can be connected either with the funnel $\mathrm{B}$ or the tube $\mathrm{C}$, or closed altogether, by means of the 3-way stopcock. A thick-walled rubber tube is attached to the 
lower end and also to a reservoir of mercury. The measuring tube $\mathrm{A}$ is first filled with air by lowering the reservoir while the tap is open. The tap is then closed and the mercury made level in the reservoir and the tube. The volume is read off. Two or 3 c.c. of strong ( 40 per cent.) caustic soda are placed in the funnel, the reservoir lowered somewhat, and the reagent allowed to run in slowly by opening the tap. Close the tap and raise and lower the reservoir a few times to spread the soda over the sides of the tube. Any carbon dioxide present is absorbed, but its concentration in air is so small that probably no diminution in volume will be noticed when the level of the mercury is again adjusted. We know, however, that it is present because lime-water left exposed becomes covered with a film of calcium carbonate, and the amount of it can be determined with a more sensitive method of analysis.

Place next in the funnel 2 or 3 c.c. of a strong solution of pyrogallol, and allow it to run into the measuring tube and mix with the caustic soda already present there. Alkaline pyrogallol is a powerful absorbent of oxygen, and there will be found to be a marked decrease in the volume of the gas. Repeat the process of raising and lowering the reservoir until no further reduction in volume occurs. The difference between this reading and that obtained after the addition of soda

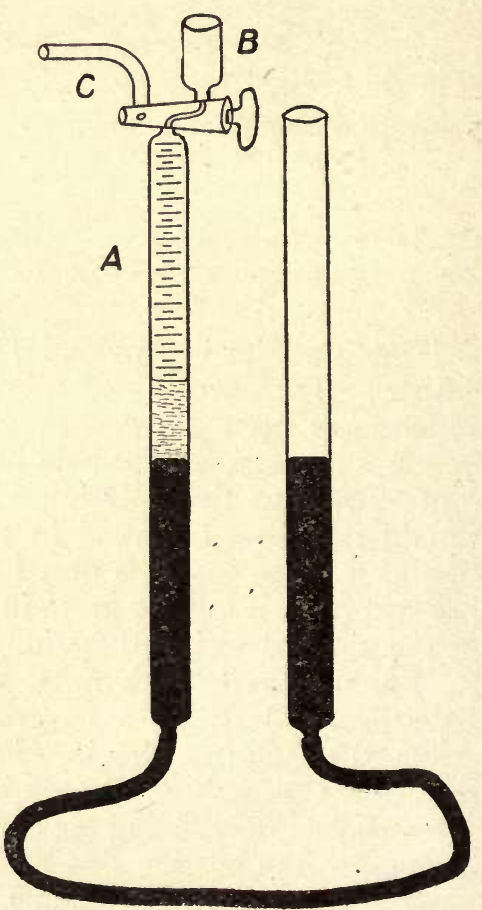

FiG. I3.-Apparatus for Analysis of Gas Mixtures. gives the volume of oxygen in the mixture, and its percentage in the air can be calculated. The remainder is nitrogen and inert gases. After use, the tube is washed out repeatedly with water, by running in through the funnel and expclling by the tube C. Finally 5 per cent. sulphuric acid should be run through.

Breathe backwards and forwards from a rubber football bladder until the asphyxial effect is too great to continue. A tube has been tied into the bladder for the purpose, and should have a piece 
of rubber tubing on the outer end in order to place a spring clip on it after the last expired air has entered.

Fill the tube $\mathrm{C}$ with mercury by raising the reservoir and opening the tap slowly. Any mercury that escapes is allowed to fall into a cup and replaced in the reservoir. Holding the bladder under the arm, press it gently while the clip is open and slip the rubber tube on the tube C., the three-way tap being closed. Open the latter and draw a sample of the gas into the measuring tube as before. Read its volume, absorb the carbon dioxide and the oxygen in turn. It will be found that there is an increase in carbon dioxide, a decrease in oxygen. Thus the combustion processes in the animal body have consumed oxygen and replaced it by carbon dioxide.

We require now to allow this expired air to be subjected to the action of a green plant in sunlight. We may take a small plant of mint in a small flower-pot. (Mint was used by Priestley in his classical experiment) Place this pot in the middle of a shallow earthenware tray and cover it with a bell jar which has an opening at the top closed with a rubber stopper through which a short glass tube passes. A piece of rubber tube with a pinchcock is fitted on the glass tube. Suck up water from the dish until the plant is immersed and the jar filled, replacing the water in the tray as it goes into the jar. The flower-pot may have muslin tied over the top to prevent the soil being washed out. Close the rubber tube by a clip. Attach the football bladder containing asphyxial air, open the clip and fill the jar with the air, pressing the bladder so as to drive some of the contents out at the bottom of the jar. The water is allowed to run into the sink. Finally pour mercury into the tray so as to cover the bottom edge of the jar.

For accuracy, a sample of the gas should be taken out of the jar after it has stood in the dark for an hour or so, and analysed. If a narrow rubber tube be attached to the tube of the jar, this tube will become filled with the gas by the pressure of the mercury when the clip is opened for a moment, and a pure sample can be drawn into the measuring tube by lowering the reservoir.

Place the jar in sunlight or bright daylight for a day or more. Possibly the heat of the sun may expand the gas so far as to drive some of it out through the mercury at the bottom, but since this mercury prevents air entering when the jar cools, it does not matter.

To obtain a pure sample of the final gas mixture, the tube connecting the jar with the analysis tube must be filled with the gas. This may be done by sucking into the analysis tube 10 or 20 c.c., and then driving the contents out through the funnel $\mathrm{B}$. The tube is then filled to a convenient level by lowering the reservoir again, taking care that more mercury is added to the tray, if necessary. On analysis, the oxygen will be found to have increased, the carbon 
dioxide decreased, or nearly disappeared. Thus the green plant in sunlight restores the normal composition to air which has been vitiated by respiratory processes.

The experiment may be repeated in the dark. There will be no increase in oxygen. Probably a decrease will be noted, together with an increase in carbon dioxide.

Chlorophyll.-To see the absorption bands in the spectrum of chlorophyll, take grass cut in short pieces, and rub in a mortar with methylated spirit. Filter and place in a test-tube in front of the slit of a pocket spectroscope. If the dark band in the red is not clearly seen, dilute with spirit.

The chloroplasts are most easily seen under the microscope in a thin leaf, such as that of a moss. Mount in water.

Formation of Starch.-Take a plant, a bean plant does well, growing in a pot. Keep it in the dark for two days. The starch will be transported from the leaves to the stem and root. Take one of the leaves, dissolve out the chlorophyll by warming in methylated spirit, and place the colourless leaf, after washing with water, in a dilute solution of iodine in potassium iodide. It will not turn blue. Allow the plant to be exposed to sunlight for a day or two, and test the leaf for starch again. It will turn blue. The iodine test should also be made with a solution of starch, made by rubbing some dry starch powder in water and pouring into boiling water.

\section{The Nitrogen Cycle}

Bacteria-Allow hay or grass to putrefy in water. Various forms of bacteria will be found on examination under the microscope. Use the highest magnifying power available.

Their forms and movements can be made more obvious by the addition of indian ink, or better, collargol, to the preparation, or by the use of dark-ground illumination.

Formation of Nitrates in the Soil.-Add half a gram of garden soil to 50 c.c. of the following culture fluid:-

Ammonium sulphate -

Potassium acid phosphate

Water -
$0.5 \mathrm{gm}$.

I litre.

Half a gramme of magnesium carbonate to the 50 c.c. is also required to preserve neutrality. After about four weeks or so, the ammonia will have disappeared, and nitrate have taken its place. The presence of nitrate may be shown as follows:- Filter the liquid. Evaporate to dryness in a porcelain basin on the waterbath. Add, with a glass rod, a drop of 0.5 per cent. solution of diphenylamine in pure, nitrate free, strong sulphuric acid. The presence of nitrate is shown by the production of aniline blue. 
Root Nodules.-A fully-grown lupin plant is dug up and the roots washed in water. Numbers of tubercles of various sizes will be seen.

\section{Salts}

The importance of calcium for physiological processes may be shown with the frog's heart. The canula of Symes is the most convenient (Fig. 14).

The apex of the ventricle (see anatomy of the heart, p. 188 below) has a tiny bent pin passed through it; a light clip is better. A

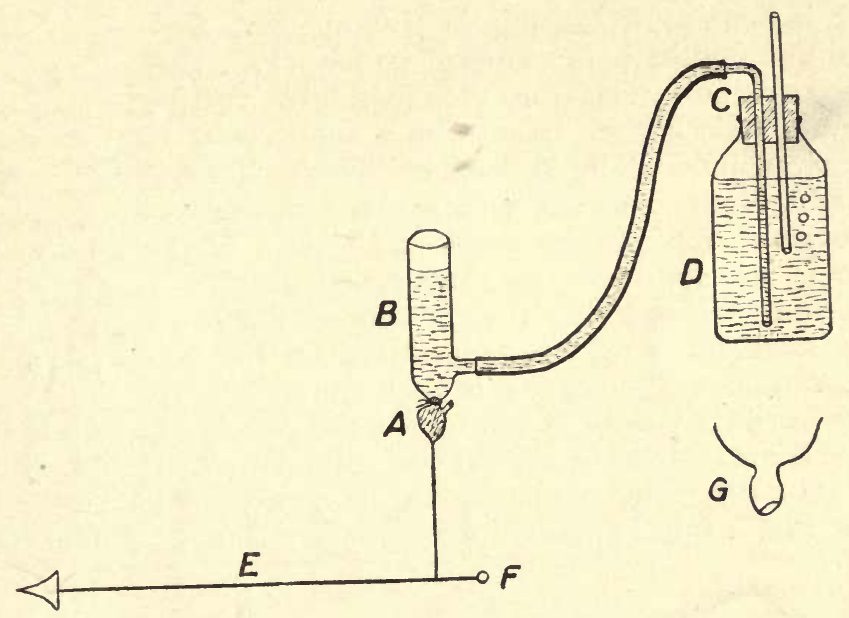

FIG.14.

A, frog heart tied on the end of the glass canula B, which has a side tube connected by rubber tubing to a siphon $\mathrm{C}$, which dips into the solution D in a Mariotte's bottle. E, a light straw lever pivoted at $\mathrm{F}$.

$G$, enlarged view of the end of the canula.

thread is attached to this clip, and to a straw lever, which magnifies the beats. A tracing may be obtained by making a paper point, fixed on the end of the lever, to write on a glazed paper gummed around a cylinder and then smoked. The cylinder is slowly rotated by clock-work or electric motor. Such apparatus is supplied by makers of physiological apparatus. The paper is smoked by a gas flame, fed with coal gas which has passed over cotton-wool on which benzene has been dropped. During the smoking the drum is rotated quickly by hand. To fix the tracing, it is removed from the drum by a vertical cut in an appropriate place and passed through a dilute spirit varnish, or a solution of paraffin wax in petrol. It is then hung up to dry. 
The heart is prepared thus: A frog, whose central nervous system has been destroyed, is laid on its back. The heart is exposed by removing the sternum (the bone in front between the fore-legs), cutting the bones which unite it to the legs with strong scissors. The beating heart will be seen in a transparent bag (the pericardium). Open this carefully and expose the heart, which has the appearance of Fig. I5. By aid of a large needle, fixed into a wooden handle by its point, a thread is passed between $B$ and $A$, taking care not to injure the auricles. The needle will be more handy if bent after heating in a flame, and then carefully smoothed by emery paper (Fig. I6). The heart is now turned up forwards, after cutting through a little fibrous band which connects it to the pericardium. The thread is tied by a loose knot around the auricles, a snip made in them as far from the ventricle as possible, the end of the canula is inserted, and the thread tied around it. By cutting through the tissues behind the heart with scissors, the heart is removed tied on the end of the canula. The siphon is now connected up,

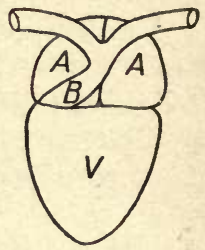

F1G. 15.

$\mathrm{V}$, is the ventricle. $\mathrm{A}$, the two auricles. $\mathrm{B}$, the commencement of the main arteries carrying blood, expelled by the contraction of the ventricle, to the body in general. and, by sucking gently at the top of the canula, the solution flows into the heart and out by the cut ends of the main arteries. The level of the solution in the canula should be 2 or $3 \mathrm{~cm}$. above the heart.

Use first a solution containing 0.65 per cent. of sodium chloride

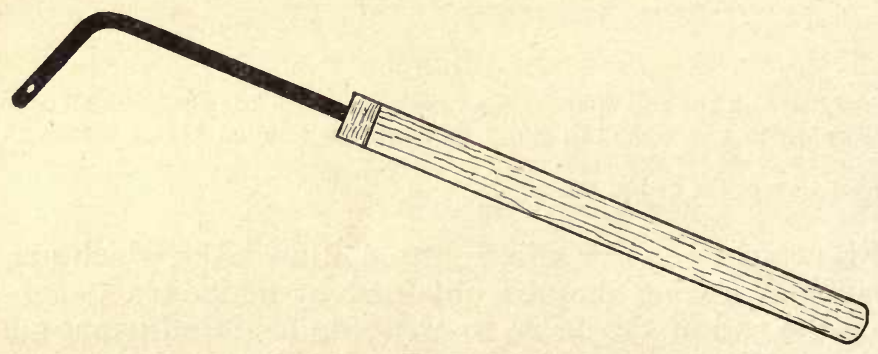

F IG. 16.-Simple Aneurism Needle.

and 0.014 per cent. of potassium chloride, in distilled water. The sodium chloride should be free from calcium, if marked results are to be obtained. The beats will be small and may in time disappear. Take next a solution containing calcium ions in addition to the sodium and potassium ions, namely, 0.65 per cent. sodium chloride, 0.0I4 per cent. potassium chloride, and 0.012 per cent. 
calcium chloride. Large and vigorous beats will be obtained, lasting for several hours.

\section{Sources of Energy}

Connect an inverted funnel, held in a clamp, to a wash-bottle containing lime-water. Bring under the funnel a piece of sugar or a bit of fat which has been set burning on iron gauze by aid of a Bunsen burner. At the same time suck the products of combustion through the wash-bottle. The lime-water becomes milky. Note that by burning food materials in air we obtain carbon dioxide and energy (heat) just as when burned (oxidised) in a more gentle way in the living organism.

\section{Alimentary Canal of Frog and Rabbit}

Opening a pithed or chloroformed frog along the middle ventral aspect, notice-

The gullet (œsophagus), leading from the back of the mouth to the stomach.

The stomach.

The narrow, small intestine, passing from the stomach and forming a few coils before opening into the broader.

Large intestine.

The large brown liver, with its gall bladder containing bile.

The pancreas, a small pale yellow organ near the beginning of the small intestine.

In a rabbit, killed by chloroform, the same organs will be better seen. The pancreas is more diffuse. The beginning of the large intestine has a voluminous sac, full of food material, attached to it; this is the cæcum, which is exceptionally large in herbivorous animals. Careful dissection will show the duct from the pancreas, opening into the small intestine about two or three inches from the stomach.

\section{Enzymes and Digestion}

Rates of Reactions.-I. Add a small quantity of silver nitrate solution to a beaker of dilute sodium chloride solution. A precipitate of silver chloride falls instantly.

2. Add I or 2 c.c. of methyl acetate to a beaker of water, containing a few drops of an alcoholic solution of phenol-phthalein. Add dilute sodium hydroxide, drop by drop, until the colour turns red. We have now an excess of alkali present. This is capable of decomposing the ester into acetic acid and methyl 
alcohol. The former combines with the sodium hydroxide, forming a neutral salt, so that when sufficient has been produced the red colour of the phenol-phthalein disappears. This disappearance will be seen to take place slowly.

The experiment may be made more accurately by taking methyl acetate and water in molecular proportions, that is, 74.I parts by weight of the former to i 8 parts by weight of the latter. Approximately, I0.5 c.c. of the ester to ro c.c. of water. Take out a sample, say 2 c.c., by means of a pipette every day or two, and determine, after dilution, the amount of acid present, by titration with standard caustic soda. The rate of change will ultimately become very slow and finally cease. By calculation of the amount of acid formed, it will be found that there is still a notable quantity of the ester left unhydrolysed, so that the reaction has come to a balanced position. Take now a sample of the mixture in equilibrium, dilute it with water to twenty times its volume. Allow it to stand for a day or two and titrate again. More acid will be present; that is, further hydrolysis has taken place.

The experiment may be performed with ethyl acetate, but the time taken will be much longer.

Hydrolysis by Enzymes. - The enzyme lipase may be used for the first experiments on this question. This catalyst acts on esters in general, and we will take the glycerol esters known as fats Lipase is found in the pancreatic juice, in the liver, and in various fatty seeds. The most convenient source is the seed of the castor-oil plant; but it is important that fresh seeds, capable of germination, be used, otherwise there may be no enzyme present. The seeds should, in fact, be obtained from a seedsman, not from a druggist.

It will suffice to rub some of the seeds with a little weak acetic acid in a mortar, after removing the outer shells by a blow with the pestle. The acid is required to form the active enzyme from a preliminary stage in the resting seed.

Add some of the paste thus obtained to an emulsion of a small quantity of olive oil in water. After vigorous shaking for a moment, take out a sample of 5 c.c. and titrate with sodium hydroxide. It will contain a small amount of acid. It is best to add the sample to 25 c.c. of methylated spirit to dissolve the oil, and then to dilute with 25 c.c. of water and add phenol-phthalein before titration.

Allow the rest to stand in a warm place, shaking at intervals, for a day or two, and then titrate again. The fat will have been partially hydrolysed to oleic acid and glycerol. Under these conditions, with excess of water, the ester is hydrolysed by the enzyme. 
To observe the opposite process of synthesis, add the paste containing lipase to a mixture of oleic acid and glycerol, to which a very small quantity of water has been added. Titrate as above at once, and again at intervals of a few days, in a warm place. The sample is best weighed in a flask, taking 2 or $3 \mathrm{gm}$. and working out the result for Io gm. in each case.

The difficulty in this experiment is that the constituents of the mixture separate from each other, so that the action is very slow, unless continual shaking is practised. But if the mixture be well shaken at intervals, an obvious synthesis, shown by decrease in the oleic acid present, should be detected.

If a polarimeter is available, an experiment showing the same facts may be made with the enzyme emulsin made from almonds. An active preparation can be bought. This enzyme acts on glucosides, in particular that kind called the $\beta$-glucosides, which are laevo-rotatory. The synthesis of the $\beta$-glucoside of glycerol can be shown thus: Take $9 \mathrm{gm}$. of glucose, dissolve by aid of heat in $6 \mathrm{gm}$. of water. Cool. Add $20 \mathrm{gm}$. of glycerol, and rub in a mortar with I or $2 \mathrm{gm}$. of emulsin. The exact quantities are not essential; the proportions given ensure the most rapid result. Take a sample of the mixture at once, say $2 \mathrm{gm}$. weighed as in the olein experiment. Add 2 or 3 c.c. of a solution of mercuric nitrate to precipitate the proteins introduced with the enzyme, make up to 50 c.c., and filter. Determine the degree of rotation. It is well to allow the solution to stand for some time after addition of the mercuric nitrate, to allow aggregation of the particles. Place the remainder in a warm place, preferably at $45^{\circ} \mathrm{C}$. Take samples every day for the first four days, then every second day. The dextro-rotation due to the glucose will become less and less, owing to the formation of the glucoside with the opposite rotation. The rotation ultimately passes to the opposite side of zero. Finally, take a sample, dilute about twenty times with water, add a little fresh emulsin, and warm for a day. Treat as before, and it will be found that the original dextro-rotation returns. Thus the same enzyme hydrolyses in dilute solution.

Ethyl-glucoside $(\beta)$ in crystals can be obtained by the aid of emulsin thus (Bourquelot): Add powdered glucose in excess to IOO c.c. of 90 per cent. alcohol, so that part remains undissolved. Then add about.a gram of emulsin and leave in a warm place, shaking at intervals, for two or three weeks. Filter. Evaporate to dryness on a water bath. Extract with a small quantity of pure, cold, dry acetone. This dissolves the glucoside, leaving glucose. Crystals separate on standing. Filter off and dry over sulphuric acid in a desiccator. The crystals will be found to form a laevo-rotatory solution and not to reduce alkaline copper 
sulphate. If a dilute solution in water be acted on by emulsin, glucose and ethyl-alcohol are formed.

Enzymes Act at their Surfaces.-Take a quantity of ground Soy beans containing urease. Add 75 per cent. alcohol, shake together, and filter off a part. Add a few crystals of urea to this filtrate and also to the suspension of the powder in alcohol. Keep in a warm place for a day, and test for the production of ammonia by aeration as described above (p. I 82). A mere trace will be found in the sample in which the filtered extract was used, arising from the slight spontaneous change of urea. In that in which the solid was suspended a large amount will be found. Thus urease acts in a liquid in which it is insoluble.

Catalytic Action.-Faraday's platinum effect may be obtained in a modified way thus: Take a little spiral of fine platinum wire. If held in forceps over the tip of a Bunsen burner and the gas turned on, it is probable that no effect will be obtained owing to the surface not being clean. Light the gas and allow the platinum to be heated to a red heat, then put out the flame by pinching the tube, and as soon as the wire has ceased to glow let the gas on again. The platinum will gradually get red and the gas be ignited. If allowed to become quite cold again it rapidly loses its activity.

The oxidation of methyl alcohol by platinum may be seen thus: Place a few c.c. of methyl alcohol in the test-tube of Fig. I 2 and in the horizontal part of the exit tube, which should be fairly wide, some platinised asbestos, that is, asbestos coated with finely-divided platinum. The bottle, empty, should preferably be immersed in ice, but cold water will serve. Immerse the test-tube in warm water and draw air through it over the platinum. The reaction will probably not commence until the latter is warmed, but will then continue of itself when the external heat is removed. If the reaction has not been too violent, formaldehyde may be detected by its smell in the condensing bottle.

Add a few c.c. of methyl acetate to water as in the experiment on p. IS9. Add paste of castor-oil seeds with dilute acid, and make just alkaline to phenol-phthalein. The red colour will disappear much more rapidly than it did in the former experiment. When this has taken place, make red again and observe the renewed disappearance, and so on. The lipase acts as a catalyst in accelerating the hydrolysis of the ester.

Model.-Some instructive experiments can be made with a schema. Take a piece of polished plate glass about $3 \mathrm{ft}$. long and 6 in. broad, such as is sold for shelves in shops. Raise one end on an adjustable support some 5 in. high. Carefully polish with chamois leather so as to remove dust. Polish also the bottom of a brass kilogram weight. Place the weight at the top of the sloping 
surface. By adjusting the height, a position can be found at which the weight will slide slowly down. This represents the course of a reaction proceeding spontaneously at a slow rate. Apply next a few drops of oil to the bottom of the weight and repeat. It slides down rapidly. The oil represents a catalyst. Note that the energy set free in the process, being given by the height from which the weight falls, is not altered by the catalyst. The form of the energy may, however, be changed. It will be noted that the weight arrives at the bottom with more kinetic energy in the presence of the catalyst than when it slides slowly down. In the latter case, there is more heat produced by friction. Another fact to be taken note of is that there is some loss of the oil "catalyst" by sticking to the glass. This represents the disappearance of a catalyst by subsidiary reactions, which often occurs.

\section{Various Digestive Enzymes}

Amylase.-Add a little saliva to some starch paste. It is quickly liquefied, and sugar will be found by boiling with alkaline copper sulphate. The blue colour with iodine will disappear.

Invertase.-Add a little yeast to a solution of cane-sugar to which a drop of chloroform has been added. This addition prevents alcoholic fermentation. Cane-sugar does not reduce alkaline copper sulphate, but the glucose and fructose resulting from its hydrolysis by invertase do so.

A similar experiment may be made with scrapings from the inside of the small intestine of a mammal.

Pepsin.-Take scrapings from the inner lining (mucous membrane) of the stomach. Add 0.5 per cent. hydrochloric acid and filter. Add two or three little cubes of hard-boiled white of egg and keep in a warm place. The egg white will be dissolved:

Trypsin.-Make a similar experiment with an extract of the pancreas, made by rubbing in a mortar with sand and 0.2 per cent. sodium bicarbonate. Add a scraping of the mucous membrane of the small intestine (containing enterokinase) to activate the trypsinogen into trypsin. Filter.

\section{Absorption}

Histological preparations of the mucous membrane of the various parts of the alimentary canal can be bought. If made in the laboratory the following method is employed:-

In order that thin sections may be cut, all tissues require to be "fixed" or hardened by some means. There are many solutions used for the purpose, and the appearance of the cells is not the same 
in all cases. The fact shows that it is not to be assumed that the minute structure of the protoplasm corresponds to that of the living state. If, however, there are things to be seen in one kind of cell that are not visible in another, we are justified in holding that something was present during life in one and not in the other.

The most generally useful fixing solution appears to be that of Bouin :-

Saturated solution of picric acid in water

Commercial formalin (40 per cent. formaldehyde)

Glacial acetic acid
60 c.c.

18 ,

2 "

The pieces of tissue should not be large, and may remain in the mixture for one or two hours. Wash repeatedly in 70 per cent. alcohol until no more yellow colour comes out. Transfer to methylated spirit for forty-eight hours and then to chloroform.

The material must be supported by being impregnated with and embedded in paraffin, which should have a melting point of $50^{\circ}$. Since paraffin is soluble in chloroform, the material may be transferred directly to melted paraffin, which should not be at a temiperature higher than sufficient to keep it melted. The tissue remains in this for one or more hours, according to size, and may be changed to fresh paraffin, since the chloroform must be got rid off. In the case of delicate tissues, it is better to pass through a solution of paraffin in chloroform before placing in the pure paraffin. If the piece of tissue is a rather thick one, it should be passed through oil of cedar-wood between the spirit and paraffin, since chloroform does not penetrate very well.

A mould is made by wrapping paper around a wooden rod, projecting beyond the end of the rod. The piece of tissue is taken out of the paraffin, by means of warmed forceps, and placed in position in the mould, so that the sections made transversely across will be in the desired plane. The mould is filled with melted paraffin and cooled as quickly as possible. The paper is taken off and the cylinder separated from the wood. Paraffin may be sliced off so as to leave the tissue at the apex of a pyramid.

Sections are cut by fixing in a microtome. This is an instrument by which the embedded tissue is advanced by fractions of a millimetre at a time and slices cut off by a razor. The rocking microtome of the Cambridge Instrument Co. is convenient. Sections should be ro $\mu$ in thickness. They must next be mounted on slides, and are usually stained in order to render their constituents more easily visible. As they leave the microtome, they are generally more or less folded or creased. To flatten them, pick up carefully with forceps and lay on warm water (not above $40^{\circ}$ ). When flat, float them on to a microscope slide by bringing the 
slide under them, lifting out and draining off the water. Press into contact with the glass by means of filter paper, and lay aside in a warm place until completely dry. Subsequent treatment will not then wash them away, except in rare cases.

Next dissolve away the paraffin by warming until it melts and pouring over some solvent; xylol is generally used. Wash away the xylol with acetone-this by alcohol, first strong and then dilute, and finally water. Various stains can then be applied. We may use : I per cent. eosin for ten minutes, rinse with water; then I per cent. toluidine blue for twenty minutes. Remove excess of stain with absolute alcohol. Drop on solution of dried canada balsam in acetone and apply cover-glass. The balsam will harden in a few hours. The use of other stains will be found in histological textbooks, such as Schafer's "Essentials of Histology."

To see the globules of $f a t$ in the intestinal epithelium, the following method is adopted: Feed a rat with butter and kill it with chloroform four hours later. Place a piece of the upper part of the small intestine into a mixture of equal parts of I per cent. osmic acid and 3 per cent. potassium bichromate and leave for ten days. Unsaturated fats reduce osmic acid and become stained black. Solvents of fat cannot be used, so that the tissue must be soaked in strong gum, and sections cut by freezing with ether spray on a simple microtome arranged for the purpose. The sections are then mounted in glycerine.

The synthesised fat can also be seen by placing a small bit of the mucous membrane in 0.5 per cent. osmic acid for forty-eight hours and then in water for a few days. A shred is placed in glycerine on the slide, a cover-glass over it, and broken up into cells by tapping the cover-glass.

\section{Voluntary and Involuntary Muscle}

The microscopic appearance of the former is best seen in the muscles of an insect, such as a wasp or beetle. Cut off the head, and divide the trunk with scissors lengthwise. Notice muscles attached both to the legs and to the wings. Take a shred of the former, and tease out with needles on a slide into separate fibres, if possible. Add a drop of the insect's blood, cover and examine with a high power. The cross striation will be seen, and, by careful focussing, the longitudinal fibrils ("sarcostyles") embedded in the "sarcoplasm."

The cells of the involuntary muscle may be seen thus: Allow a small piece of intestine to macerate in $\frac{1}{8}$ per cent potassium bichromate for two days. Hold it in water on a microscope slide with forceps and fray out with a needle. The cells separated in 
this way may be examined with a high power, after covering the preparation.

The urinary bladder of the frog exhibits the arrangement of smooth muscle well. Distend it with alcohol by means of a pipette. When hardened, cut out a piece of a few millimetres square. Stain. Mount in glycerine.

\section{Contractions of the Frog's Stomach}

Cut with scissors a ring from the frog's stomach by two parallel transverse cuts. Pass a bent pin through the ring and hang it up on a rod over the lever used above for the heart (p. 187). Pass through the ring another hook and connect it to the lever by a thread. The muscle will usually at first be in tonic contraction. Note that running warm $\left(25^{\circ}\right.$ to $30^{\circ} \mathrm{C}$. $) 0.7$ per cent. sodium chloride over it causes relaxation. If the muscle has already relaxed somewhat, the first effect of the warm saline may be to excite a contraction, but this is followed by a marked relaxation. A series of rhythmic contractions sometimes follows.

The tonic contraction will slowly give way without warming, especially if a small weight, I or $2 \mathrm{gm}$., be attached to the lever so as to stretch the preparation slightly. After a time there may be slow rhythmic contractions and relaxations.

Apply induction shocks by twisting the end of a fine copper wire, attached to one terminal of the secondary coil, around the upper pin and a wire from the other terminal to the lower pin. Note the slow contraction.

\section{Secretion}

Examine under the microscope a thin bit of the rabbit's pancreas. Note the granules in the cells.

Make sections of the salivary gland as described above for intestinal mucous membrane. Study the general arrangement. Vertical sections through the frog's skin show typical simple glands.

The disappearance of granules in the act of secretion may be seen in the living stomach of the newt, as described by Langley and Sewall (Journal of Physiology, vol. ii., p. 286). Feed a newt with small earthworms or by introducing with a pipette some diluted white of egg into the stomach. In twenty-four hours the digestive process is over, the glands have assumed the resting appearance and are ready for renewed secretion, being full of granules. Take another newt three hours after feeding. The granules in some of the cells have nearly disappeared; in others 
there are still some remaining, forming an inner granular zone around the lumen.

The observations are made by pithing the animal, opening the body cavity, dividing the stomach along the greater curvature and pinning one half of it over an opening in a plate of cork or thin wood, with the muscular coat uppermost. It may be convenient to pin the newt on its side. The muscular coat is sufficiently transparent to allow the deeper ends of the glands to be examined under a fairly high power. If care be taken to avoid loss of blood, it is possible to see the circulation around the glands. The muscular coat may be snipped off by a fine pair of scissors at some spot, if difficulty be found in seeing the glands.

Flow of Water.-One of the forms of osmometer described on p. I7 I above is filled with a strong solution of congo-red, a fine glass tube bent over at the top is inserted and the osmometer immersed in distilled water. After a time, drops of dye solution will issue from the upper end of the tube and may be collected.

Electrical Change.-Although this may be regarded as a somewhat difficult experiment for the student, it has much importance in the general theory, and, at all events, it should be shown as a demonstration.

A sensitive high resistance galvanometer is required, such as the Broca pattern made by the Cambridge Instrument Co. It should be made as sensitive as possible by very careful adjustment of the controlling magnet, so as to produce a long period of oscillation. A spot of light from a lamp is reflected by the mirror attached to the moving magnet and received on a divided scale. If an arc lamp is used, the spot will be bright enough to be visible at some distance.

The tissue to be investigated must be led off by non-polarisable (and equipotential) electrodes. The most convenient pattern is that in which mercury and calomel are used. Take a small widemouthed bottle, fitted with a paraffined cork through which three glass tubes pass, two of these ending a short distance below the cork, the other is longer. One of the two short ones is bent at a right angle outside the bottle. The other short one is fitted with a piece of rubber tube closed by a clip and is used for filling. The third tube has a short piece of platinum wire fused into the lower end, which is then sealed and contact made with the platinum by pouring in a little mercury and dipping into it a fine copper wire. This tube passes down into a layer of mercury of a few millimetres depth at the bottom of the bottle and makes contact with it by the platinum wire. Some calomel, together with a little mercury, is rubbed in a mortar to a paste 
with 0.7 per cent. sodium chloride if the electrodes are to be used on frog's tissue, or 0.9 per cent. if for mammals. This paste is shaken up with a larger quantity of the solution and poured into the bottle so as nearly to fill it. A piece of cotton spirit lamp wick has previously been pushed into the bent tube, and the solution is run in until it escapes from the end of this tube. The clip is then closed. The wick may be cut to any size or shape, especially if a little kaolin is put on it, according to the organ or tissue to be investigated. Two of these electrodes will be needed.

It will be found that when any two points on a living tissue are

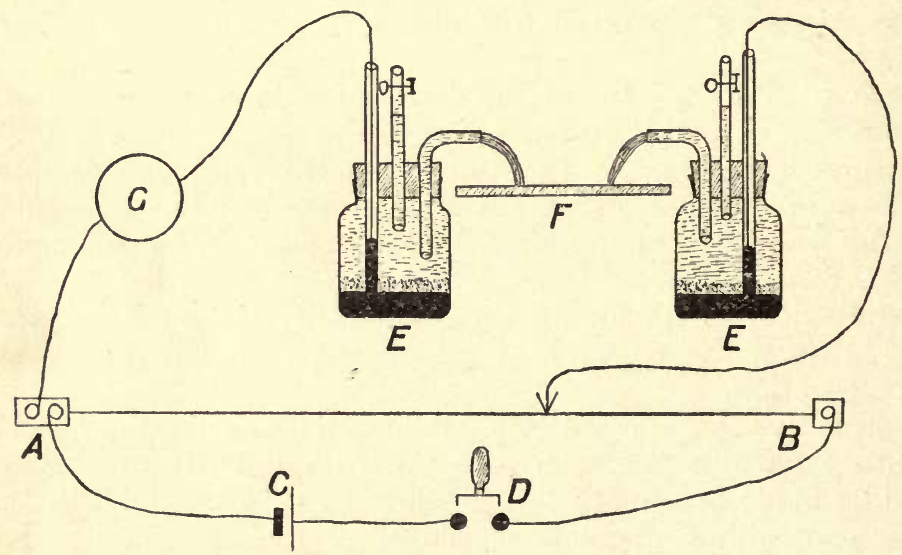

FIG. 17.-Circuit for Experiments on Electrical Changes.
A B, slide-wire.
C, battery.
D, key.
$\mathrm{E}$, electrodes.
F, tissue.
$\mathrm{G}$, galvanometer.

connected to the galvanometer there is usually a deflection, which may be great enough to send the spot of light off the scale. This is partly due to differences of potential in the tissue itsclf, partly to unavoidable inequalities in the electrodes. To diminish the latter as far as possible, it is well to keep the electrodes connected together when not in use. This is done by placing a wire with its ends one in each of the mercury tubes and connecting the bent tubes with a piece of india-rubber tubing filled with salt solution.

But in any case a means of balancing the electro-motive force present in the tissue is necessary. An equal and opposite electromotive force is put into the circuit by an adjustable contact on a wire through which a current is flowing. The slide-wire used previously will serve. The whole circuit is arranged as in Fig. I7.

It is convenient to have an adjustable resistance connected across 
the terminals of the galvanometer so as to be able to adjust the sensibility for various purposes. If the direction of the current in the slide-wire is not such as to oppose the E. M. F. of the tissue, the connections of the battery must be reversed, or a commutator may be interposed in the circuit.

The skin of the frog is the simplest secretory structure in which the electrical change of activity can be seen. The electrodes are placed one on each leg between the knee and the ankle. The sciatic nerve is prepared on one side, a ligature tied around it, and the nerve cut on the central side of the ligature. It is laid on two wires, best of platinum, connected with the secondary coil of the induction apparatus and the current thrown in when the galvanometer has been brought to zero. The nerve should be raised out of the wound and laid on the stimulating electrodes held in some support. A pillar of plasticine is the simplest. Since the skin of one leg only is stimulated, that of the other acts merely as a conductor. If both nerves were stimulated, the changes in one would neutralise those in the other, owing to the opposite directions in which they are connected to the galvanometer. The actual electrical change seen is sometimes of a complicated nature, consisting of more than one phase. The explanation of the whole is not altogether clear, but we need only observe the fact of a change in the gland cells.

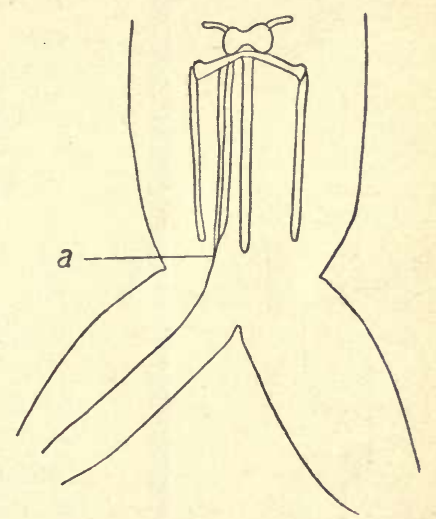

F1G. 18.-Position of the Sciatic Nerve.

$a$, place in which the incision is made.

Since the stimulation of the sciatic nerve causes the muscles to contract, and this would interfere with observation of the glandular effect, we must first give the frog an injection of the arrow poison, curare, by means of a hypodermic syringe inserted under the skin of the back. About 3 drops of a 0.05 per cent. solution will usually be sufficient, but different samples of curare vary in strength. The solution should be made fresh. In small doses it prevents stimulation applied to the nerve from reaching the muscle, but does not affect the glands. When the frog is completely paralysed it is pithed or beheaded.

The sciatic nerve is found by making an incision through the skin between the end bone of the spinal column, urostyle, and the pelvic arch on one side at the extremity of the former (as in Fig. I8), 
the frog lying on its lower side. Cutting carefully through the tissues under the skin with fine pointed scissors, the nerve will be found. Pass a thread under all the nerve trunks seen and, raising them by the thread, not yet tied, follow up with the scissors to the place where they leave the spinal column. Tie here and cut between the ligature and the bone. Dur-

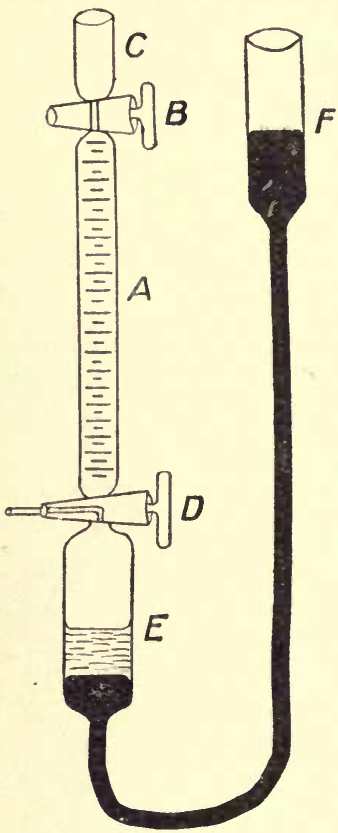

FIG. 19.-Vacuum Pump for Blood Gas Experiments.
A, graduated tube.
B, stopcock.
C, cup.
D, three-way stopcock.
E, reservoir.
$\mathrm{F}$, mercury vessel. ing the experiment keep the skin moist with 0.7 per cent. sodium chloride.

\section{Respiration}

Trachee of Insect.-Any small piece of tissue cut from the interior of an insect and spread out on a microscope slide in 0.7 per cent. saline will show the branching system of tubes containing air.

Hamoglobin.-The carriage of oxygen by the red corpuscles of the blood can be shown by a simplified vacuum pump made by a glass-blower. Fig. 19 shows the pattern, which will be found useful for many purposes.

With the stopcock is open to the cup $\mathrm{C}$, and $\mathrm{D}$ making communication between $A$ and $E$, raise the mercury reservoir $F$ until a little mercury has entered c. Close $B$ and lower the reservoir until the mercury leaves $\mathrm{E}$, that is $760 \mathrm{~mm}$. below $\mathrm{E}$. There is now a Torricellian vacuum in $\mathrm{A}$ and $\mathrm{E}$.

Take about io to 15 c.c. of blood, which should be fresh, and either defibrinated by stirring with a feather, or prevented from clotting by the addition of a small amount of powdered potassium oxalate. Sufficient will be otained from a rat killed by cutting its throat. If defibrinated, it will need straining through muslin to remove bits of fibrin.

Allow a known volume of this blood to run into the vacuum by putting it in the cup C and turning the stopcock slowly. It will be seen to froth and to become more crimson in colour. Raise the reservoir, after closing the stopcock, until the blood, neglecting the froth, just fills the vessel E. Then turn the stopcock D so that the blood is driven out. Collect it in a small bottle. Bring the mercury reservoir into connection with $\mathrm{A}$ again, and in such a position that 
the level of the mercury is the same in both. Read the volume of the gas.

Add I or 2 c.c. of strong sodium hydroxide through the cup after lowering the reservoir. The volume of the gas will diminish, owing to absorption of carbon dioxide. Next add in the same way I or 2 c.c. of pyrogallol. Nearly the whole of the rest of the gas will be absorbed showing that it is oxygen. The small residue is nitrogen.

We now want to see whether the blood which has lost oxygen can take it up again and give it off to a vacuum. We must first wash out the pump by running in water and expelling it from the side by the stopcock D, until it comes away colourless, finally rinsing out with 0.9 per cent. sodium chloride.

Now rotate the bottle containing the dark blood so that a thin film is formed over the interior. The blood becomes bright red again. Repeat the process of removing the gas by the pump, noting how much blood is used. We obtain practically the same volume of oxygen as before, taking account of the respective quantities of blood used.

To be satisfied that it is the corpuscles and not the plasma that has this function, the experiment may be repeated with serum or plasma. A little oxygen may be given off if hæmolysis has occurred and the serum is red. Colourless serum can be obtained by allowing blood to clot and to stand until the serum has exuded from the clot and can be collected in a pipette.

Absorption Spectrum.-After exposure to oxygen there are two bands in the yellow of the spectrum, best seen in very dilute solution of hæmoglobin in water. Addition of a drop of blood from the finger to a test-tube full of water will serve. Add more water if too concentrated. The addition of a few drops of a reducing agent, such as ammonium sulphide, and warming, changes this spectrum to one of a single band. Shaking with air brings back the original two bands for a shat time.

It may be thought more convincing to remove the oxygen by the pump. Take a dilute oxy-hæmoglobin such as shows the twobanded spectrum in a tube of the same diameter as that of the pump. Fill the pump with mercury, and then run in gently 2 or 3 c.c. of the solution. Observe with the spectroscope whether the two bands can be seen. If so, close the stopcock and lower the mercury vessel. Drive out the gas given off through the cup C. It will not be entirely reabsorbed during the operation. Repeat until the spectroscope shows the single band. A small relative amount of the oxy-hæmoglobin is sufficient to show the double band.

Carriage of Carhon Dioxide.-To show that hæmoglobin also carries carbon dioxide, it is necessary to remove the serum from the 
blood by washing the corpuscles with 0.9 per cent. sodium chloride. Centrifuge some defibrinated blood. Pour off the serum. Shake up the deposit with saline and centrifuge again. After repetition for two or three times there will be practically no serum left. The reason for this procedure is that the sodium bicarbonate in the serum gives off carbon dioxide to a vacuum, although it does not to the tension of this gas in the air-cells of the lungs.

The final suspension of corpuscles in saline is first put in the pump and the gas removed. Then the process to which blood was subjected in the former experiment is repeated, except that the corpuscles are subjected to an atmosphere of carbon dioxide in the bottle instead of to air. It will be found that much more carbon dioxide is obtained in the pump afterwards than could be dissolved in the water present.

The carbon dioxide may be made in a Kipp generator, and should be passed through sodium bicarbonate solution in order to stop spray containing hydrochloric acid.

\section{Stimulation of Respiration by Carbon Dioxide}

Breathe from a football bladder a gas mixture containing about Io per cent. of carbon dioxide, together with more oxygen than serves to make up that displaced by the carbon dioxide. The respiration will be found to be quickened and deepened, while the feeling of "want of breath," as after running upstairs, will be experienced. It may be found that less carbon dioxide will give the result better.

The oxygen is most conveniently obtained from a cylinder of the compressed gas, but it may be made by the usual process of heating potassium chlorate with manganese dioxide. It should be washed by passing through caustic soda. A sample of the mixture as breathed should be analysed in the apparatus used for the green plant experiment (p. I 84).

\section{Oxidation}

Autoxidation.-Expose some benzaldehyde and also linseed oil in shallow dishes to the air. Note that crystals of benzoic acid appear in the former, and that the latter becomes hardened as in varnish.

No effect is to be seen with sugar or lactic acid exposed to the air. That they are not oxidised to carbon dioxide and water can be shown by leaving a small quantity in a large closed bottle for some days and determining the carbon dioxide content of the gas in the bottle by analysis. Or, more simply, insert a perforated rubber stopper with two tubes, and draw air, freed from carbon dioxide by first passing through a wash-bottle of caustic soda, through the bottle and then through lime-water. 
Peroxides. - Hydrogen peroxide oxidises lead sulphide to sulphate, which the oxygen of the air does not. But hydrogen peroxide alone does not oxidise lactic acid. The addition of a catalyst, such as iron, results in its oxidation, as shown thus :

Using the apparatus of Fig. I2 (p. I82), put a dilute solution of lactic acid into A and lime-water into B. The air entering should preferably have passed through caustic soda. No carbon dioxide is formed. Add hydrogen peroxide (the commercial 20-volume solution will serve). Again suck air through. There is still no formation of carbon dioxide. Add I or 2 c.c. of a dilute solution of ferrous sulphate; carbon dioxide is produced.

Peroxidase.-Instead of ferrous sulphate as above, take a fresh mixture of lactic acid and hydrogen peroxide and add grated horse-radish root. Carbon dioxide is evolved.

Guatiacum Reaction.-Take an extract of horse-radish in water, add to some of it in a test-tube a drop of freshly made solution of guaiacum resin in alcohol (guaiaconic acid is better). It is precipitated by the watery solution, so that any change of colour is difficult to see. Accordingly, add alcohol to dissolve the deposit. It is not blue. In another test-tube, after the addition of guaiacum, add a small quantity of hydrogen peroxide. Alcohol then added will dissolve the blue oxidation product of the guaiacum.

Solution of guaiacum dropped on the cut surface of a potato is blued at once, so that the peroxide is already there.

The cut surfaces of potatoes, apples, and other fruits turn brown on exposure to the air. This is because there is a compound in them which turns brown on oxidation.

The peroxide in these cases is only formed when free oxygen is present. Place a potato with a cut surface uppermost in a widemouthed glass bottle, through whose cork three glass tubes pass, one to the bottom of the bottle, another leads from the top to a Bunsen burner, while the end of the third opens just above the potato and is, at its outer end, connected by a rubber tube with a clip on it to a little funnel containing guaiacum solution. Pass coal gas through the bottle and light it at the burner. The peroxide previously present is soon used up. Allow the guaiacum to drop on the potato. No blue colour will be seen. Turn off the gas and empty out the potato into the air. It rapidly turns blue.

Reduction.-Add sufficient solution of methylene blue to quite fresh milk to give a distinctly blue colour. Then a small quantity of formalin. The blue colour disappears more or less rapidly. The reaction does not take place if the milk has been boiled, so that it is due to an enzyme. Milk that has undergone bacterial change reduces methylene blue without the presence of an aldehyde, since some of the bacterial products serve the same purpose. 


\section{CHAPTER III}

THE articulated arm of a skeleton (the arm can be bought separately) should be examined to realise how movements are brought about by muscles. Articulated bones, various useful models of organs and so on, can be obtained from Deyrolle of Paris.

Attach a cord in the place, say, of the biceps muscle, at one end to one of the bones of the fore-arm near the elbow, at the other end to the bone of the scapula (shoulder-blade) just above the shoulder joint By looping up the cord so as to shorten it the elbow is flexed. If the cord is attached at one end to the ulna where it forms the prominence of the elbow, at the other to the bone of the upper arm, it extends the elbow joint or straightens out the arm, since it is attached on the opposite side of the fulcrum to that of the biceps. It represents the triceps.

The "Contraction" of Muscle.-Take a pithed or beheaded frog, cut across above the pelvic girdle with strong scissors. Remove the remains of the viscera in the posterior part. Seize the skin at the cut edge with forceps and the pelvic girdle with the left thumb and forefinger. The skin can now be pulled off, turning inside out in the process. The muscles of the legs are exposed, and, by the application of electrodes connected to an induction apparatus to each in turn, its action can be observed.

Tension in Muscle.-The string or thread used for the experiment described in the text must not be too strong, otherwise sufficient weight required to break it may not be available.

Spring.-Suitable springs may be bought at the ironmonger's. Those of about $\mathrm{I}$ in. diameter and 4 or 5 in. long do well. But the exact size is immaterial.

Nerve-Muscle Preparation.-Skin the legs of a pithed frog as above. Prepare the sciatic nerve as on p. 199, but dissecting it free down to the knee-joint. Pass a thread under the tendon of the calf muscle where it is attached to the heel. Tie and divide the tendon below the knot. Separate the muscles from the leg-bone up to the knee. Cut across the thigh-bone at the middle, and remove the muscles attached to the lower part, taking care not to injure the nerve. Finally cut across the leg-bone just below the knee. 
The preparation may be most conveniently mounted on the cork plate of a "myograph," which is a flat board with a bellcrank lever attached. Put a pin through the end of the femur into the cork. Tie the thread on the tendon to the upright of the lever. Lay the nerve on electrodes, which may be held in place by means of a lump of plasticine. A weight of 10 or $20 \mathrm{gms}$. is hung on the lever near its axis.

For stimulating electrically, some form of induction coil is used. The secondary coil is wound on a bobbin separate from the primary coil, so that it can be placed at different distances for adjusting the strength of the stimulation. There is an automatic interrupter to give shocks in continuous series. Such coils are sold by dealers in physiological or electro-therapeutical apparatus.

Electrical stimuli of moderate intensity do no damage to the nerve, whereas it is difficult to avoid killing it by other forms. Hence these latter can only be applied once at a particular spot. The end of the nerve furthest away from the muscle must be used first. A gentle tap with the back of a scalpel serves as a mechanical stimulus, a crystal of salt as a chemical one, a heated wire as a thermal one.

Notice that the excised muscle does work in raising a weight. It may be said that the weight falls again, so that no actual external work is done. But when it is raised, a support may be slipped under it, and, after the muscle has relaxed on cessation of stimulation, the thread to which the weight is attached, which will now be lax, may be shortened, and then the next stimulation raises the weight still further. With patience the process may be repeated many times until the muscle becomes fatigued. An automatic "work-collector" on the principle of the ratchet wheel is constructed to perform the operations described above.

\section{Formation of Acid}

Cut across a muscle which has not been stimulated, and press a piece of neutral litmus paper on the cut surface. It will become blue. Thus the reaction is alkaline. Stimulate a muscle until it ceases to respond and repeat the test. It will be acid, turning the paper red. A more elegant form of the experiment is to inject under the skin of the back of a frog a few drops of a strong solution of the dye, acid-fuchsin. This is colourless in alkaline solution, so that when examined next day the muscles have their usual yellowbrown colour. Excise one and stimulate it until it is fatigued. On cutting across, it will be found to have become red, the change being most obvious when compared with a similar muscle which has remained at rest. 


\section{Effect of Length of Fibres}

The simplest way to see the fact that more work is done when the fibres are longer is to load the muscle with increasing weights and to determine the height of the contraction produced by a single induction shock in each case. The product of the weight by the height gives the work. If the actual value in gram-centimetres is wanted, it is of course necessary to measure the relative distances, from the axis of rotation of the lever, of the attachment of the muscle, of the weight and of the tracing point of the lever. For the present purpose we only require to compare the different values of work with each other. The point may write on a vertical smoked paper. Move the surface so as to produce a short horizontal line after each weight has been added and then stimulate the nerve. An arc of a circle is drawn. The addition of each weight, say $5 \mathrm{gm}$. at a time, stretches the muscle a little more, so that the curve starts from a lower level. The lever may be brought to the horizontal position again by moving it in the slot of the myograph. The tracing should be fixed, as described above (p. 187), and when dry can be measured with compasses. It will be found that the work done increases with the length of the muscle up to a certain point, and then begins to decrease as the muscle becomes abnormally stretched.

\section{The Structure of Voluntary Muscle}

This has been studied previously (p. 195), but should be brought into relation with the fact of the preceding experiment. If only insect muscle has been examined, it would be well to make a preparation of the voluntary muscle of the rat or mouse. Mount it in a little serum from the animal itself. Observe under the highest power available, focussing the surface carefully.

\section{Effect of Temperature}

This has also been observed on the tonic contraction of the frog's stomach. A further case is described under the heart (p. 225). To study the action on the voluntary muscle requires special methods, and is not easy to observe correctly. Note its significance in relation to changes of surface tension between sarcostyle and sarcoplasm.

\section{Production of Tetanus}

On a nerve-muscle preparation observe the height of the contraction produced by a single shock, and that in which the shocks are given as quickly as possible after one another by opening and 
closing a key in the primary circuit, and also by using the automatic interrupter, which has a much more rapid rate of vibration.

\section{Heart Muscle}

There are certain facts which require delicate and exact apparatus to demonstrate on voluntary muscle, but can more easily be observed on the slowly contracting heart muscle of the frog.

It is necessary to be able to make use of a frog heart which does not beat spontaneously. This is attained by the application of a ligature in such a way as to cut off the sinus from the rest of the heart (Stannius' ligature). Pass a thread between the aortæ and the auricles as described above (p. 188). Turn the heart forwards. Bring the ends of the thread around the auricles and tie so that the knot presses on the place where the auricles join the sinus, marked by a whitish line. In addition to cutting off the impulses from the sinus (see below, p. 225), it is probable that the vagus nerves are stimulated also. The stoppage of the heart does not last long as a rule, so that the experiments on it must be done as quickly as possible. Have everything ready before the application of the ligature. Cut out the heart and fix it to the cork plate by a pin through the aortic bulb. Pass a tiny bent pin through the apex of the ventricle and connect it by a thin thread to the lever, which is fixed above the heart. If the same lever as that used in previous experiments with the heart be used, there must be a prolongation to the opposite side of the axis, since the contraction of the heart pulls downwards in the present case.

The "Staircase."-This requires that the muscle should have rested for some time, so that it should be the first experiment made.

Make electrodes of fine wire, the two wires being attached by sealing wax at about $2 \mathrm{~mm}$. apart, leaving about a centimetre free at the end. Arrange these so that they touch the ventricle gently, supporting them on a lump of plasticine. Arrange the stimulating coil so that single shocks can be given by a key in the primary circuit. Do not use shocks stronger than can be comfortably felt on the tongue. Allow the tracing point of the lever to rest lightly in contact with a thinly smoked surface, which is at rest. Give a series of stimuli, each following the other just after the effect of the previous one is over, and moving the surface by hand a few millimetres between each. The height of the contractions will increase for the first few beats and then remain stationary.

"All-or-Nothing."-When the steady stage has been reached, vary the strength of the stimulus. It will be found that the beats 
will be all of the same height if the stimulus is strong enough to stimulate at all. This is because the whole of the heart muscle contracts at each beat, all its cells being in functional connection with each other, contrary to the case of the voluntary muscle, where a varying number of fibres can be set into activity.

The Refractory Period.- Using the weakest stimulus found in the last experiment to be effective, first produce a beat, and at various stages in its course, judged by the eye, apply a second stimulus. This will be found to have no effect until a certain stage in the relaxation period has been reached.

If the laboratory is warm, the contraction may be so rapid that the experiment is difficult. If so, lumps of ice may be placed on the cork around the heart. The water from the melting of the ice must be prevented from reaching the heart by a little wall of plasticine.

In case the heart has recommenced beating by the time this last experiment is arrived at, the first stimulation of the preceding method is omitted, using instead of the artificial contraction, the natural one, and applying the second stimulus at various points of the natural beat, which may be slowed by ice as before. 


\section{CHAPTER IV}

\section{Spinal Frog}

DESTROY the brain of a frog by inserting a pointed bit of wood into the skull cavity from the gap between the back of the skull and the spinal column, which can be felt by taking the frog in a cloth and bending the head downwards. A large pin may be found more convenient to destroy the brain, since it penetrates the skin more easily; but the bit of wood should afterwards be inserted to stop bleeding.

After a few minutes' time, reflex movements can be elicited by pinching the toes, dropping dilute acid on the skin, applying a hot wire or an electrical stimulus. All of these are effected without conscious sensation, since the brain is absent.

\section{Central Nervous System}

The general arrangement of the nerve centres in the frog should be observed at this stage. Take a frog which has lain in methylated spirit for a day or two. Remove the bony covering of the skull by inserting one point of a strong finely pointed pair of scissors through the membrane between the skull and the spinal column. Also remove the arches of the vertebræ posteriorly from the same place. Note the nerves connecting the nerve centres to all parts of the body; especially the optic nerves conveying impulses from the eyes and the fact that the sciatic nerve, which we have seen to cause muscular movement, is given off by the spinal cord.

\section{Nerve}

Structure.-Tease a piece of fresh sciatic nerve of the frog in 0.7 per cent. saline, obtaining the fibres as long and straight as possible. It is not very easy to separate individual fibres uninjured. Note the clouble contour of each fibre, due to the highly refracting medullary sheath, and the interruptions of this sheath at intervals (Nodes of Ranvier). The material of the medullary sheath may 
be seen forming highly refracting masses of curious shapes where it has escaped from the cut ends of the fibres. To see the axis cylinder, the contents of the medullary sheath may be dissolved away by the action of chloroform.

Electrical Change in Nerve.-The arrangement of apparatus is the same as that for the skin glands (Fig. on p. 198 above). Owing to the high resistance of nerves, the two sciatic nerves of a frog may be laid side by side on a glass slide. One electrode is placed on the surface of the preparation, near the middle, the other one on the cut end, which has been killed for a length of a few $\mathrm{mm}$. by touching with a hot wire. Electrodes for stimulating are placed near the opposite end. The leading-off electrodes are first made to touch each other. If there is any potential difference between them, it is compensated by the slide-wire. It will be small in any case. There will be found to be a fairly high potential difference between the injured end of the nerve and the normal surface, such that the longitudinal surface is positive to the end. This should be absent when two symmetrical places on the longitudinal surface are led off, provided that neither of them is injured. This may be ensured by allowing the nerve to lie in saline solution in the cold for a day before examining it; all the injured fibres will then be dead. To find which way the spot of light moves when either electrode is positive, attach a bit of zinc to a copper wire from one terminal of the galvanometer and a plain copper wire to the other. On placing the zinc and copper in dilute salt solution, the copper becomes positive to the zinc. The galvanometer must be shortcircuited by a low resistance in order to avoid too violent a deflection.

Having compensated the so-called "current of injury," or "resting current," stimulate by rapid induction shocks with the automatic interrupter of the coil. A deflection will be obtained in a direction opposite to that of the current of injury.

Nerve Unexcited by Light or Sound Waves.-The nerve-muscle preparation is used. No contraction is obtained if a beam of light be allowed to fall on the nerve by placing it in front of the window, screened by a piece of black cardboard, and suddenly removing the screen. Neither will the sound of a bell excite the nerve trunk.

\section{Receptors of the Skin}

Histological preparations of human skin, showing various forms, may be bought. If fresh skin can be obtained, vertical sections may be made by the method described above (p. 193). Receptors are looked for in the projecting papillæ underneath the outer "epidermis." 


\section{Taste-Buds}

These will be found in sections across the "papilla foliata" of the rabbit's tongue. These are two small oval areas, one on each side of the back of the tongue.

\section{Olfactory Cells}

Take a small piece of the mucous membrane of the upper part of the nasal cavity of the frog, beneath the olfactory lobes projecting from the front of the brain. Place in I per cent. osmic acid for a few hours. Soak in water for two or three days. Tease in dilute glycerine, or break up by tapping on the cover-glass. Look for narrow cells with brush-like outer ends. These are the smell receptors.

\section{Heat and Cold Spots}

Explore the skin of the back of your hand for these, using a simple instrument made thus :-Draw out a piece of half-inch glass tubing to about $\frac{1}{8}$ th in. Cut it at the narrow part. Cement with sealing wax into the end a short piece of thick copper wire filed to a small rounded point. Wrap flannel around the tube so that the temperature may remain fairly constant for some minutes. For heat spots, put water at $40^{\circ} \mathrm{C}$. into the tube and allow it to rest gently on various places of the skin. When one is found which is sensitive to heat, mark it with red ink for future identification. For cold spots, put finely broken ice into the tube and proceed in a similar way, marking the spots black. They will be found to have different situations from the heat spots.

Stimulate both kinds with induction shocks by placing on them finely pointed electrodes. The sensation from each will be its own appropriate one, so that, if stimulated at all, the sensation is always the same. A temperature which feels distinctly warm to the heat spots does not affect the cold spots, and vice versa. But a temperature sufficiently high to stimulate the nerve fibres themselves will produce a sensation of cold from a cold spot. Thus, while $15^{\circ}$ produces a sensation of cold from a cold spot and none from a heat spot, one of $45^{\circ}$ produces a sensation of heat from a heat spot and cold from a cold spot. This paradox is due, of course, to the operation of the law of specific sensation, as applied to the fact that the receptors for temperatures above that of the skin and below it are separate organs. 


\section{Hearing}

Preparations of the cochlea are difficult to make. They may sometimes be bought. That of the guinea-pig is the easiest to prepare on account of its size. The method used is as follows :The part of the skull containing the petrous bone is cut out with bone forceps. A hole is filed at the top of the cochlea, which is seen as a conical eminence. The preparation is then placed in fixing fluid, and the bone afterwards decalcified by immersion in a solution made by dissolving I gm. of phloroglucin in nitric acid with the aid of heat and diluting to 100 c.c. with water. The object of the phloroglucin is to counteract the effect of the acid in causing the tissues to swell. Wash well and transfer to alcohol, gradually increasing in strength. Then through cedar oil and paraffin as usual. The transition from one liquid to the other must be gradual, by using mixtures with the preceding one, since the organ of Corti is very readily broken up. Sections are cut through the axis of the spiral, so that it is not possible to obtain many from one preparation.

The resonance of a stretched membrane may be seen by taking a triangular piece of sheet india-rubber, and attaching two of its sides to strips of wood by screwing down on to each of the strips a second one of the same size. One side is firmly clamped to the edge of the bench, the other held in the hand and used to stretch the membrane. Sand is dusted on to the membrane, and a pitchpipe or other similar source of a musical note sounded near. By adjustment of the pitch of the note, and by different degrees of tension on the membrane, a particular place may be found which vibrates in sympathy with the note, as shown by scattering of the sand. Suppose that this place is near the wider end when a low note is sounded, then by raising the pitch a narrower region will resonate.

\section{Photo-Receptors. The Eye}

Visual Purple.-Keep a frog in the dark for a day. Kill it by pithing in a room lit only by a sodium flame or a dim photographic red lamp. Excise the eye-balls. Cut each one into a front and a back half by means of a razor. Put the latter half into a dish of 0.7 per cent. sodium chloride. Seize the outer coat at its cut edge with forceps and shake the retina loose. If it does not come free from the place where the optic nerve enters, a pointed scalpel or fine scissors may be used to cut it free. Taking the dish into ordinary light, the beautiful crimson colour of the retina will be seen. It will become bleached more or less rapidly according to the intensity of the light. 
Anatomy of the Eye.-Take the fresh eye of an ox. Note-

The transparent cornea in front, continuous with the opaque sclerotic.

The entrance of the optic nerve, opposite the cornea.

The coloured iris, with the aperture (pupil) in the middle.

Divide the eye into front and back halves by a razor. Note-

In posterior half-

Gelatinous vitreous humor in posterior chamber.

Thin transparent retina, covering the

Black choroid coat.

Entrance of the optic nerve and its continuity with the retina.

In anterior half-

The coating membrane of the vitreous humor attached to the front of the choroid and holding the lens in a capsule behind the pupil.

Remove the lens, noting its elastic nature.

Note the aqueous humor, filling the anterior chamber between the cornea and the lens.

Take another eye and freeze it in a mixture of ice and salt. When solid, divide it by a section at right angles to the preceding one, namely, perpendicular to the surface of the cornea and passing through the entrance of the optic nerve.

Study the relative positions of the parts as they become visible on thawing. Note especially the way the capsule of the lens is attached to thickened ridges of the choroid close behind where the cornea and sclerotic meet; and that the iris is also attached to these "ciliary processes." The fibres of the ciliary muscle in them are firmly attached at the junction of the cornea and sclerotic, and when they contract they pull forward and relax the suspension of the lens, so that the latter takes up more or less its natural more spherical form. Thus the focal length is altered to accommodate for near objects.

Image on the Retina.-Expose the back of the eye in a dead rabbit by cutting away the bone and other tissues. Place an incandescent lamp two or three yards in front of the eye, and examine the back of the eye with a lens. If the pigment of the choroid is not too dense, a minute inverted image of the filament is seen on the retina. It is easily seen if an albino rabbit, which has no pigment in the choroid, be taken. It is possible, with care, in an ordinary rabbit to cut out a little window in the sclerotic, and to brush away the pigment with a camel hair brush wetted with 0.9 per cent. saline.

When the image is seen, place a microscope slide in front of the 
cornea, so as just to avoid touching it. Drop 0.9 per cent. saline into the gap between the surfaces. The image will disappear, because the curved refracting surface is no longer present. Remove the glass slide, the image reappears.

Structure of the Retina.- The posterior half of the eye of a rabbit, after removal of the vitreous humor, is fixed, and sections cut of a strip of the retina and sclerotic together.

Place a piece of retina in I per cent. osmic acid for a few hours, and then in dilute glycerine for a day or two. Take a small fragment and tease it with needles in dilute glycerine. Further separation of the constituents is brought about by tapping the cover-glass. Many of the points in the structure of the retina can be made out by aid of the figures in the text-books of histology.

\section{Receptors for Position}

The small transparent fresh-water mollusc, Cyclas, has a statocyst attached to the ganglion in the foot, and can be examined in the living state under a low power of the microscope.

Expose the cartilaginous skull of a skate or dog-fish.

Notice the position of the semi-circular canals, one set on each side, after removal of the roof of the skull and the brain. They are contained within a mass of cartilage behind the eyes. Carefully slice away the cartilage until the three canals become easily visible. Note their position in the three dimensions of space. 


\section{CHAPTER V}

\section{The Central Nervous System}

AlLow a frog to lie for a day or two in methylated spirit. Remove the muscles from the surfaces of the arches of the spinal column. Open the neural canal by dividing the membrane between the skull and the vertebræ. Cut away, bit by bit, the bony roof of the skull by means of a narrow-bladed but strong pair of scissors. Remove the arches of the vertebræ in the same way. The spinal cord is usually concealed more or less by soft material, which can be gently removed with a blunt pair of forceps.

Notice the brain in the skull, the spinal cord in the vertebral column, continuous with one another.

The Brain.-In front, two elongated masses forming half of the brain. Each has an anterior small part and a large posterior part. The former is the centre for smell (olfactory lobes); the latter, the cerebral hemispheres; on their surface the cortex.

Between the posterior ends of the cerebral hemispheres the thalami appear, and from them an optic lobe (the mid-brain), forming a rounded eminence, projects on each side.

A narrow band, the cerebellum, lies transversely just behind the optic lobes. It is very small in the frog.

The rest of the brain forms the bulb, and contains very important nerve centres. Its dorsal aspect presents a triangular hollow, the fourth ventricle.

Raise the brain carefully, beginning anteriorly and cutting through the nerves passing from it through the skull. Notice the ventral aspect of the structures mentioned above.

In the spinal cord note the number of nerves given off, and that each arises by two roots. The dorsal root contains the afferent or sensory fibres, the ventral root the efferent or motor fibres.

Sympathetic System.-Turning the frog on its back, remove the viscera and find the sympathetic trunk on each side of the main arterial trunk, the aorta. It is a slender cord with ganglia (enlargements containing nerve cells) at intervals. Note the delicate nerves uniting these ganglia to the spinal nerves, most easily seen in the case of the long nerves to the hind-legs. 
Make a similar preparation of a mammal, such as a rat. Note that the cerebral hemispheres and the cerebellum have grown so much as to cover over the other parts, which can only be seen by raising or removing the former. The optic lobes, on the other hand, are relatively small, and are represented in the mammal by the corpora quadrigemina, four little eminences.

Examine in a museum the remains of various extinct vertebrates, such as the Plesiosaurus. Note the small size of the skull in proportion to the rest of the body. Compare the state of affairs in the cat or man, and realise that the law of progress is that the prizes go to the wise, and that the main factor in evolution is the development of the nervous system.

The cell-bodies of the spinal neurones are well seen by taking a piece of the fresh spinal cord of the ox. With the point of a scalpel, take out a small piece of the central grey matter from the more ventral broader part. Tease in 0.9 per cent. saline and put on a cover-glass, with a bit of hair to protect the cells from pressure. Look for large branching cells.

Another way by which fixed cells are obtained is to allow a small piece of the grey matter to macerate for a day or two in very dilute chromic acid in a small stoppered bottle. Shake. Allow to deposit, and mount some of the deposit as above. This preparamay be stained and preserved by mounting in dilute glycerine.

Similar preparations should be made of the cortex of the cerebral hemispheres and of the cerebellum of the rat.

\section{Spinal Reflexes}

Some observations were made above (p. 209) on the spinal frog. A more detailed study is to be made here.

Having made the preparation as before, hang up the frog to the edge of the table by a pin through its jaw.

Gently pinch one of the toes. Note the movement.

Apply a very small bit of filter-paper dipped in acetic acid to the skin of one flank or thigh. The leg will wipe it away.

Stimulate the toes of one side by a stimulus that can be adjusted in strength, such as the induced currents from the induction coil. Note that the number of muscles engaged in the reflex increases with the strength of the stimulus. Also that the time the movements last after the stimulus has ceased is longer with strong stimuli.

Dip the foot in weak sulphuric acid of progressively increasing strength (0.1, 0.2, $0.3,0.4$, and 0.5 per cent.), dipping the foot in water as soon as the reflex has been observed. Note that there 
is a pause before the foot is withdrawn, and that this is longer, the weaker the acid.

Find a strength of acid that invariably results in withdrawal of the foot after a moderate time. Then pinch firmly the opposite foot at the same time as the acid is applied. The reflex is stopped or takes much longer to show itself (inhibition).

\section{Inhibition}

That an action in progress can be stopped by nervous influences is most clearly shown by stimulating the vagus nerve, and thus stopping the beats of the heart. It is somewhat difficult to dissect out this nerve in the frog, but it may be stimulated at its origin from the bulb in the following way:-

Kill a frog by cutting off the brain with scissors just behind the eyes. Then cut through the spinal column between the shoulder blades and destroy the posterior part with a pin. The bulb is thus isolated, with the vagus nerves passing from it to the heart.

Expose the heart and connect the apex of the ventricle to the lever as on p. 207 above. Put electrodes into the exposed surface of the bulb where the front part of the head has been cut off, fixing them to the cork by means of plasticine. Stimulate at first with weak currents, increasing the strength until the heart stops.

The fact may also be shown by stimulating the vagus nerves in their course from the sinus to the auricles. The electrodes are placed on the white crescentic line marking the junction of these chambers. Only a weak stimulus must be used, otherwise the muscle itself is stimulated to rapid contractions. 


\section{CHAPTER VI}

Model of the Circulation.-Prepare with glass and rubber tubing, etc., an arrangement such as that indicated in Fig. 20. A is the bulb of a Higginson syringe, to be obtained from the druggist, the valves being removed. At one end a valve $B$ is connected. This consists of a glass tube, represented separately at $\mathrm{M}$, closed at one end, and having a hole blown in the side about $2 \mathrm{~cm}$. from the closed end. The edges of this hole are smoothed in the flame. This is pushed through a rubber cork fitting the end of a wider tube, which is drawn out at the other end to fit $\frac{3}{16}$ in. soft rubber tubing. A piece of sheet rubber is tied over the hole, with its free edges just meeting opposite the hole, the threads being in the situations represented. When water is pressed through this valve it escapes between the rubber and the cork. Any pressure in the opposite direction closes the hole by forcing the rubber into it. The bulb with its two valves represents the heart. $\mathrm{D}$ is a mercury manometer connected by a $T$-tube in the course of the current. This $T$-tube is continued by a 4 -ft. length of $\frac{3}{16}$ in. rubber tubing (C), of moderately thin walls, representing the arterial system. E is a screw pinchcock to represent peripheral resistance. $\mathrm{F}$ is a wide glass tube, fitted with rubber corks through which short glass tubes pass, and filled with small pieces of sponge, loosely packed. This represents the capillaries. A short rubber tube connects this with a 6-in. length of the inner tube of a bicycle tyre (G), which has rubber corks similar to those of $\mathrm{F}$. There is another mercury manometer $(\mathrm{H})$ to indicate the pressure in the veins, which are represented by that part of the model between $\mathrm{F}$ and $\mathrm{K} . \mathrm{K}$ is a valve similar to $\mathrm{B}$, and represents the mitral valve of the heart, whereas $B$ represents the aortic valves. $\mathrm{K}$ is, of course, connected in such a way that water can pass it from the vein to the bulb and not the opposite way. $\mathrm{G}$ and $\mathrm{K}$ are connected by a short piece of rubber tubing, so that they can be disconnected for convenience of filling the system with water. $\mathrm{L}$ is a $\mathrm{T}$-tube joined to a funnel, for the purpose of running in more water. It is closed by a pinchcock.

With this schema a large number of instructive experiments can be performed. The following are some of these :-

Disconnect the tube $\mathrm{C}$ from $\mathrm{F}$, and $\mathrm{G}$ from $\mathrm{K}$. Insert a glass 


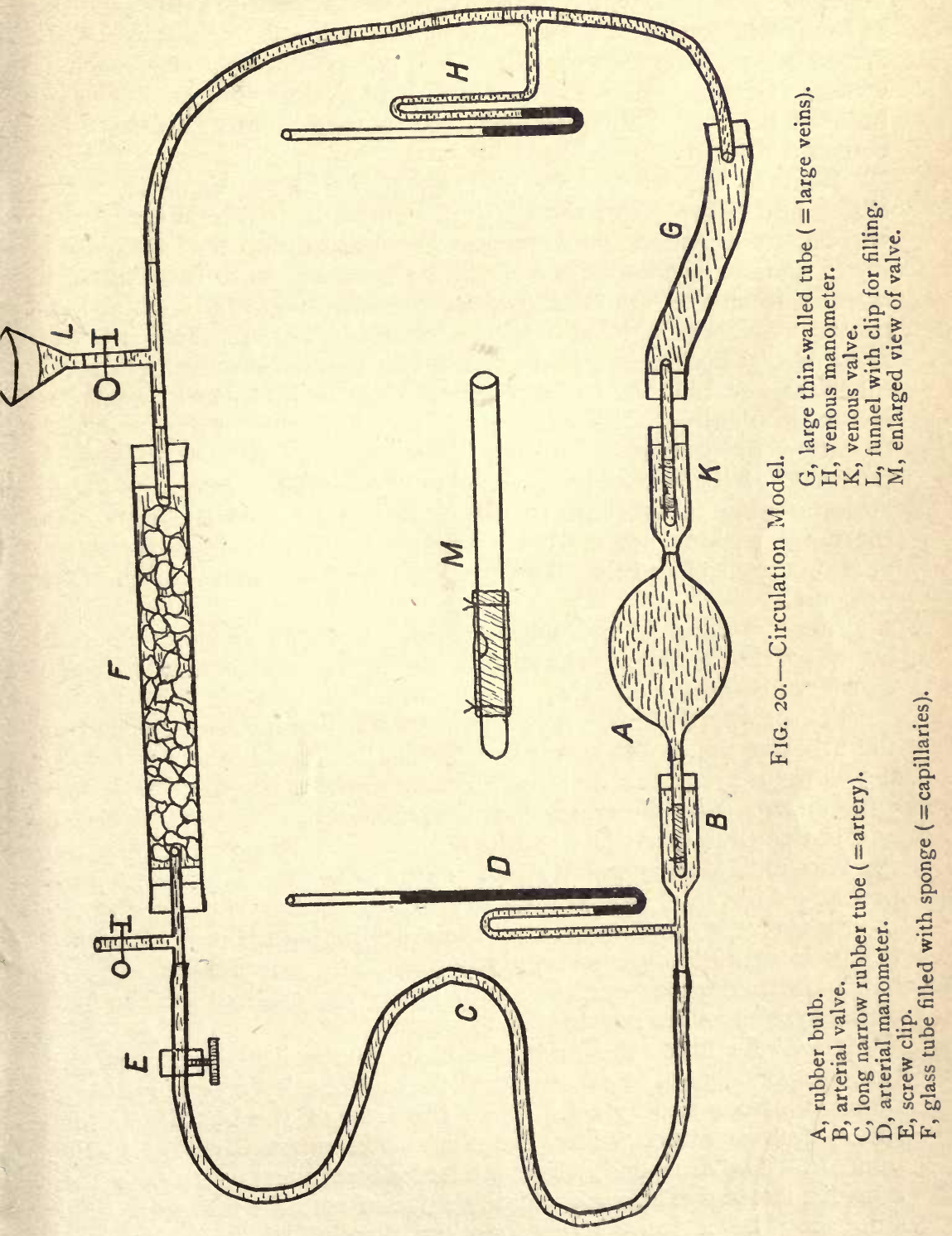


jet into the end of $\mathrm{C}$. Immerse the end of $\mathrm{K}$ in a bowl of water. With the clamp E widely open, compress A gently with the hand at regular intervals of about one or two seconds. Air will first be driven out and then water, which will escape from the end of $\mathrm{C}$ in spurts. Gradually close E, continuing the regular squeezing of the bulb as before. The spurts will be converted into a more or less constant stream.

Remove the jet and connect up again to F. Open the screw clamp and pump water through until all air is driven out, and then slip on, under water, the connection between $\mathrm{G}$ and $\mathrm{K}$.

Periodic compression of $\mathrm{A}$ will be seen to cause the valve $\mathrm{B}$ to open, $\mathrm{K}$ to close. On releasing the pressure the suction of the bulb draws water through $\mathrm{K}$, while $\mathrm{B}$ closes. The mercury in both manometers oscillates greatly, while the mean pressure in D is not much higher than in $\mathrm{H}$ (vaso-dilatation). Close next the screw clamp gradually. Note the rise in $\mathrm{D}$ and the nearly complete cessation of oscillations in $\mathrm{H}$ (vaso-constriction). If $\mathrm{G}$ is only partially distended at rest, owing to the amount of water in the system being insufficient to fill it, it will be noticed that when the compressions are started, particularly with a high resistance at E, G becomes less full. The arterial tube is stretched by the high pressure.

After running in more water under pressure through $\mathrm{L}$, it can be shown that a higher arterial pressure is maintained with a similar degree of compression of A.

Take away the manometer $\mathrm{H}$ with its $\mathrm{T}$-tube and connect up the tubes at the gap. Insert the $T$-tube between $\mathrm{E}$ and $\mathrm{F}$. Pumping as before, note that the pressure beyond $\mathrm{E}$ is low when the screw clip is narrowed. Next insert the manometer D between $\mathrm{F}$ and $\mathrm{L}$, and notice that there is not much difference of pressure between the two ends of the capillary region, but that if $\mathrm{E}$ is widened both pressures rise, thus indicating a rise in the capillary pressure.

Restore the manometers to their original positions. Arrange that $\mathrm{G}$ is well filled, pump regularly and then compress $\mathrm{G}$ with the hand, continuing the pumping as before. Decrease of the capacity of the system raises the arterial pressure.

It will facilitate the more accurate comparison of pressures if the compression of $\mathrm{A}$ is arranged to be done by a piece of wood, attached at one end by a hinge to the base of the board on which the system is fitted. This piece of wood passes over A, resting upon it. The free end, when pressed down, meets a stop of such a height as to give an appropriate degree of compression to $\mathrm{A}$. This stop may be a long screw passing through the wooden base. The rate of the compression is kept constant by the beat of a metronome. 
If this regularity of compression is ensured, the effect of viscosity may be investigated. Notice the height of the arterial pressure when water is used. Then fill the system with 7 per cent. gum arabic, without altering any of the adjustments. Repeating the experiment, the pressure will be much higher. An instructive way of doing the experiment is to disconnect between $\mathrm{F}$ and $\mathrm{L}$, and put the funnel into the end of the tube connected with $\mathrm{K}$. Run in gum solution through the funnel while the bulb $\mathrm{A}$ is being compressed regularly. As the water is displaced the pressure in D will steadily rise.

Seven per cent. gum arabic has a viscosity about equal to that of blood. It is easily made by placing the necessary amount in water the day before it is wanted. The solution will need straining through fine muslin or glass wool. To find out how much solution is wanted, the system is filled with $v_{v}$ ater and then emptied into a graduated cylinder.

The whole apparatus should be taken to pieces after use and the parts kept separated. The sponges should be taken out of their tube and dried.

\section{The Circulation in the Frog's Web}

Take a piece of thin wood, such as the lid of a cigar box, about 6 in. by 2 or 3 in. Near one end make a hole about half an inch in diameter.

Anæsthetise a frog by an injection of urethane. One minim of a 5 per cent. solution for a medium-sized frog $(25 \mathrm{gm}$.), under the skin of the back, will be about the correct dose. When motionless, lay the frog, belly downwards, on the board. Tie threads to two of the toes, place a glass slide over the hole, and draw the web over it by fixing the threads into notches in the end of the wood. Do not stretch it tightly. Keep the frog moist by wet filter paper on its back, and the web by occasionally dropping water on it. Examine with low magnification, and note the network of blood vessels below the layer of pigment cells.

The arteries are recognised by the rapid current of blood in them; they run mainly towards the free edge of the web. The direction of the flow is from larger to smaller vessels.

The capillaries, in which the arterial branches end, form a close network in which the current is slow.

The veins are formed by union of capillaries. The blood-flow is more rapid than in the latter, but not so fast as in the arteries. The direction of flow is from smaller to larger vessels, and mainly away from the free edge of the web.

Next take a triangular bit of cover-glass, place it on the web 
and examine with a higher power. Note the difference in thickness of the walls of the arteries, veins, and capillaries. The walls of the latter appear merely as thin lines. Also note the rapid stream in the middle of the arteries, the slower one near the walls; this latter may be seen to contain many leucocytes. The flow in the capillaries is much slower; the red corpuscles are often distorted in shape, but recover again. They are thus elastic.

\section{The Heart of the Sheep}

Obtain a sheep's heart from the butcher, if possible with the lungs attached. This is often called the "pull."

The anterior or ventral surface is recognised by the groove filled with fat, which marks the boundary between the two ventricles: Note that the right ventricle is softer than the left. The pulmonary artery comes from the former nearly in the middle line. The aorta is behind it and will be cut.

The two great veins from the body, superior and inferior venæ cavæ, have been cut. Note that they enter the right auricle, so that the blood passes into the right side of the heart and from the ventricle into the lungs. Find the veins returning from the lungs to the left auricle, their blood thence flowing to the left ventricle and to the aorta.

Open the right auricle and cut away most of it. Pour water into the orifice leading to the right ventricle. Note the flaps of the tricuspid valve floating up.

With scissors, one blade between two of the flaps of the valve, cut through the wall of the ventricle towards the apex. Then upwards again along the septum, but not quite into the pulmonary artery. Cut across the pulmonary artery, and tie into it a glass tube a few inches long and as large as will fit in. Pour water into this tube, and observe from below the closed semilunar valves. Note also the fine cords attaching the flaps of the tricuspid valve to the walls of the ventricle.

Open up the left auricle and ventricle in a similar way, noting especially the openings of the arteries feeding the heart (coronary arteries) behind the aortic valves.

Look for the band of special conducting tissue passing from auricles to ventricles. Branches will be found on both sides of the septum between the ventricles. They stain deeply with iodine on account of the large amount of glycogen they contain.

\section{Blood}

Mount quickly a small drop of blood from the finger. Examine at once with a high power. Note :- 
The red corpuscles, mostly collected in rouleaux (strings of corpuscles stuck together by their flat sides). They appear, when seen singly, to be of a faint yellow colour.

The colourless corpuscles or leucocytes. If the cover-glass be touched with a needle, they tend to stick to the glass, while the red corpuscles are driven about by the current.

Look also for fibrin filaments in the clear spaces.

If the preparation is to be observed for any length of time, the cover-glas; should have been painted around the edge with vaseline, as described above (p. 166).

An interesting preparation showing the fibrin produced in the process of clotting may be made thus:-Make a preparation as above, and leave for ten minutes. Remove the cover-glass, run a few drops of distilled water over the slide to dissolve the corpuscles. Drop on a moderately dilute solution of Spiller's purple. Leave for a few minutes. Wash with distilled water. Allow to dry and mount in balsam. A network of fibrin will be seen, having lumps of precipitated colloid at the junctions of the filaments (so-called "platelets"). (Schafer.)

\section{The Kidney}

Sections of the kidney of the mouse may be prepared in the ordinary way. Notice the tufts of blood vessels in capsules in the cortical part. These tufts can be made out better in sections of a kidney of which the blood vessels have been injected with a coloured material. Such sections can be bought.

\section{The Salts of the Blood}

Pass a 0.7 per cent. solution of pure sodium chloride in distilled water through a frog's heart preparation, as described above (p. 187). The beats will become weak or cease. Take Ioo c.c. of the solution and add 3 c.c. of 0.1 molar (= I. I per cent.) calcium chloride. Pass through the heart. The beats increase in size, but the heart tends to become tonically contracted, shown by incomplete relaxation between the beats. Take another I00 c.c. of the sodium chloride solution, add 3 c.c. of the calcium chloride solution and also 6 c.c. of 0.1 molar ( $=0.75$ per cent.) solution of potassium chloride. Pass through the heart. The beats become vigorous with normal relaxation. Pass again the pure sodium chloride. When the beats have become small, take IoO c.c. of the solution and add 6 c.c. of the potassium chloride solution. No improvement results. Hence all these three cations-sodium potassium, and 
calcium - are required to maintain normal beats. This is Ringer's solution. (See P, p. 209).

Make the Ringer's solution just acid to methyl-red $\left(\mathrm{IO}^{-4} \mathrm{~N}\right.$ in $\mathrm{H}$-ion) and perfuse. On the other side, make it alkaline to phenolphthalein $\left(\mathrm{IO}^{-9}\right)$ and perfuse.

Hydrogen-ion Regulation.-Makea solution of sodium bicarbonate of 0.25 per cent., which is about the usual concentration in the blood. Put 5 c.c. of this into a stoppered bottle, add a drop of o. I per cent. neutral red. Fill the bottle with the first air expired after a deep inspiration. Shake together. The colour is orange-red. Fill the bottle with alveolar air (p. 176). The colour becomes red. Run in carbon dioxide from a Kipp generator. The colour is crimson. Replace by repeated changes of atmospheric air. The colour goes back through red to orange and yellow. Bicarbonate solutions lose carbon dioxide to air, becoining more and more alkaline. Thus the hydrogen-ion concentration is regulated by the proportion of carbon dioxide to bicarbonate.

\section{Vaso-Motor Effects}

Prepare a frog for observation of the circulation in the web (p. 22I). Carefully dissect out the sciatic nerve in the thigh, avoiding injury to the blood vessels. Pass a thread under the nerve Select an arteriole for observation, and measure its diameter by the ocular micrometer. Lift up the sciatic nerve and cut it. Note the widening of the arteriole. Pick up the peripheral end with forceps; tie a thread just beyond the points of the forceps. After waiting a minute or two, measure the arteriole again and then stimulate the nerve with induction currents of moderate strength. The arteriole narrows. It may be necessary to tie down the leg or pin it to the cork, owing to the muscular contractions produced by the stimulation. But the better way would be to curarize the frog, as described above (p. 199).

Apply a drop of dilute adrenaline solution ( $\mathrm{I}$ in $\mathrm{I0,000}$ ) to the web. The arteriole becomes almost obliterated.

Take the web of the opposite side and allow a drop of a saturated solution of chloroform to fall upon it. The arterioles dilate, and a more copious flow through the capillaries is seen.

Wash away the chloroform, and when the circulation has become normal again, apply a drop of a solution of histamine (o.I per cent.). If the dose is correct the capillaries may dilate, but not the arterioles.

Adrenaline is sold in convenient tabloids by Burroughs \& Wellcome, under the name of "hemisine." Histamine tabloids are sold as "ergamine." 


\section{Action of Drugs on the Heart}

Arrange a frog heart for perfusion (p. 187). Add a small amount of adrenaline to the Ringer's solution used for perfusion (one part in several thousands is active). The heart beat is increased in rate and in strength owing to stimulation of the accelerator (sympathetic) nerve-endings in it.

Add a very small quantity of acetyl-choline to another part of the Ringer's solution (one part in a million or less). The heart is stopped.

Prepare another frog for recording the effect on the heart of stimulation of the vagus (p. 217). Having obtained slowing or complete inhibition, drop I per cent. solution of atropine sulphate in Ringer's solution over the heart. Stimulate the vagus again. No effect will be seen, nor even if the junction of the sinus with the auricles be stimulated. The endings of the vagus nerves are paralysed.

\section{The Beat of the Heart}

Expose the heart of a frog as previously described.

Note that each beat consists of a cycle, beginning in the sinus, followed by the auricles, ventricle, and bulbus in turn.

To show that the beat is initiated by the sinus, which thus controls the rate, Gaskell's method of local warming may be used. Take two pieces of copper wire of about $\frac{3}{16}$ in. diameter. Solder flexible electric light cable on to each, so that they may be joined up to a storage cell, placing a key in the circuit. Wrap thread around one of the wires up to half an inch of the free end, and then tie the wires together. Bend each wire outwards at the free end, so that there is a gap of about a quarter of an inch. Solder across this gap a bit of fine German silver wire. When the circuit is closed, the fine wire is heated. Take care that it does not get hot enough to melt the solder. It will be better to have an adjustable resistance in the circuit.

Having prepared a frog with the heart joined to the lever as on p. 217 , fix the heating wire on a pillar of plasticine so that it is near to the sinus, but not touching it. On closing the circuit, the rate of the heart beats increases markedly. Now move the heating wire to lie just above the apex of the ventricle; the fine wire may be bent concave to escape the suspension thread. When the heating current is put on there is no change in the rate if the sinus is protected. But the ventricular beats decrease in amplitude. This last fact is another aspect of the temperature coefficient of surface energy which we saw exemplified in the effect of heat on the tonus of smooth muscle (p. 196). 


\section{CHAPTER VII}

\section{Dividing Nuclei}

To see the phenomenon as it actually takes place, the blood corpuscles of the newt are favourable objects.

Newts are kept without food for three months. They are then fed with small worms, cautiously at first. After about ten days there are a large number of young red corpuscles in the blood and in the process of division. These cells are almost devoid of hæmoglobin, round and with large nuclei. The complete process takes about half an hour. For further details, see the paper by Jolly (Archives d'Anatomie Microscopique, Tome vi. (1904), p. 455).

The Development of the Frog should be watched. Collect some of the spawn in the spring, placing it in water in shallow dishes. Green weeds should be supplied. Notice the changes, first to the tadpole, then the appearance of legs, the disappearance of the tail, and the attainment of the form of the adult frog. Many details will be noticed and sketches may be made. The very early stages are best observed under a simple lens.

\section{Ova and Spermatozoa}

The ovary of the frog is most easily made out just after the breeding season, after the full-grown ova have been shed.

Remove one of the ovaries and place it in 0.7 per cent. saline. Make an incision into it. It contains a cavity. Upon the walls of this cavity are round eminences of various sizes. These are ova in various stages in development.

Tease out a bit of ovary in 0.7 per cent. saline. Examine with a low power. Note that there are many ova much smaller than those seen with the naked eye. They are granular spherical cells, with a clear central part.

The spermatozoa can be seen by opening one of the testes and pressing out some of its contents upon a slide, mounting in water and examining with a high power. They appear as long motile filaments. More typical spermatozoa can be obtained from the rat, and consist of a small head and a long motile tail. The 
contents of the testis may be pressed out into 0.9 per cent. sodium chloride.

\section{The Structure of a Flower}

Examine a buttercup or other large regular flower. Note the four sets of organs :-

I. The outer green calyx, which formed the covering of the flower in bud.

2. The corolla, consisting of petals, the most conspicuous part of the flower, usually coloured.

3. The stamens or male elements. Each consisting of a stalk or filament, ending in a knob, the anther. Tease an anther in water and examine with the microscope. Note the pollen grains.

4. The pistil, consisting of separate carpels in the buttercup. At their upper ends they have a sticky surface, the stigma; in the interior of the lower part, the ovary, will be found the ovules.

\section{Process of Fertilisation in the Plant}

This is difficult to follow in all its stages in one species of plant.

The penetration of the pollen tube into the stigma and style can be seen in the evening primrose.

Hold the stigma and style between the finger and thumb of the left hand. Moisten with a drop of water and cut successive sections with a razor, wetted with water. Spread them out in water with a needle and examine with a low power. The triangular pollen grains send out a tube from one angle into the tissue of the stigma.

The entrance of the pollen tube into the ovule can be made out in Peronica serpyllifolia, the speedwell, common in meadows.

Take a flower from which the corolla has just dropped. Dissect out the small ovary, and open one of its two chambers with needles in a drop of water. The use of a lens may be necessary. A mass of ovules is removed from the interior and teased apart. Put on a cover-glass and search for an ovule showing the entrance of the pollen tube. The further progress of the pollen tube can be seen better if dilute glycerine be run under the cover-glass and allowed to soak into the ovule and make it transparent. 


\section{INDEX}

Absolute zero, 12

Absorption from stomach, $7 \mathrm{I}$

- of amino-acids, 75

- of fats, 74

- spectrum of hæmoglobin, 201

Accelerator nerves, I 49

Accessory factors of food, 6 I

Accommodation in eye, 115

Acetic acid, 45

Acid of stomach, 75

- production in muscle, 205

Acid-fuchsin, 205

Acidic dyes, 37

Acidity, 26

Acidosis, 89, 143

Acids, 25

- strength of, 26

Activation of oxygen, 90

Active mass, 68

Adaptation, 158, 159

Addison's disease, 136

Adjustment to outsice changes, 120

Adrenaline, I31, I47, 150, 224, 225

Adrenals, 131

Adsorption, 15

Adsorption by charcoal, 168

- by enzymes, 70

- compounds, 36

- in catalysis, 69

- of basic and acidic dyes, 37, 39

- of gases by charcoal, 86,87

- of ions by colloids, $34,3^{6}$

Advantage of raised temperature, IOI

Afferent nerves, 121

After-action in cortex, 130

Air-sacs, 89

Alanine, 48

Alcohol and guinea-pigs, 157

Alcohols, 43, 64

Aldehydes, 43

- in reduction processes, 92

Aldoses, 43

Alimentary canal, 66, I 89

- movements of, 78

Alkalinity, 27

"All-or-nothing," 97, 105

- in heart muscle, 207

- in nerve, 130
Amino-acids, 45

- as food, 46

Ammonia, 45

- as source of nitrogen, 57

- test for, 181

- to neutralise acid in blood, I44

Amœba, sources of, I64

Amœboid movement, 6

Amphoteric substances, 46

Amplitude of vibrations, 108

Amylase, 70

- of saliva, 193

Anabolism and catabolism, 155

Anæsthesia of nerve fibres, I3 I

Anaphylactic shock, I4I

Anion, 24

Anode, 24

Antherozooids, 153

Anti-bodies, 141

Anti-scorbutic factor, 6I

Antiseptic method, 57

Aorta, 133

Appetite, 80, 82

Arc-lamp, 163

Arsenious sulphide, 178

Arteries, 133

Arterioles, I 33

Articulated bones, 204

Aseptic method, 57

Association neurones, 122

Asymmetric carbon atom, 48

Atoms, 4, 6, 24

Atropine 150, 225

Auditory nerve, number of fibres in, IIO

Auricle, 132

Auriculo-ventricular bundle, 150

Autoxidation, 90, 202

Axon, 122

BACTERIA, 56, 15 I, 158, 186

Bacterial changes in large intestine, 78

Balance, 167

- of excitation and inhibition, 125

Balanced salts, 142

Basal metabolism, $6 \hat{3}$ 
Bases, strength of, 26

Basic dyes, 37

Basilar membrane, i ro

Beat of the heart, 225

Beri-beri, 6I

Bicarbonate of blood, 224

Biceps muscle, 93

Bile, 74

- pigments, 135

Bladder, 99

Blood, 85, 135, 222

- circulation of, 132

- supply of kidney, 140

- vessels, permeability of, I37, I 40

Blushing, 146

"Bonds," 44

Bones and joints, 93

Bouin's fixing fluid, 194

Bound energy, 13

Boundaries, 8

Boyle's law, 22

Brain, blood supply to, 146

- of frog, 21 5

Breathing, 88

Brownian movement, 3, 6, 166

Bundle of His-Kent, I 50

Butter, 30

Calcium, 59

- and clotting, 142

- function of, 142

- on frog heart, 187

Calomel electrodes, 197

Calorie, 63

Cambrian period, 60

Camera and eye compared, I I 4

Cane-sugar, 44, 72

Capacity factor, 14

Capillaries, I 33

Carbohydrate cycle, 73

Carbohydrates, 42

- digestion of, 70

Carbon atom, properties of, 42

- compounds, 4I, 42

- test for, $18 \mathrm{I}$

- dioxide absorption, 88

- - action of, on respiration, 202

- - and hæmoglobin, 88

- - in expired air, 182

- source of, $4 \mathrm{I}$

Carboxyl, 43

Carriage of carbon dioxide, $20 \mathrm{I}$

- of oxygen by hæmoglobin, 200

Catalase, 55,9 I
Catalysis model, 192

Catalysts, 66

Catalytic action of platinum, 192

Cathode, 24

Cation, 24

Cell, 3

- membrane, 9, 15,65

- sap, 20

Central nervous system of frog, 209, 215

- - of mammal, 216

Cerebral cortex, 127

- hemispheres, 103, 120

Changes in permeability, 29, 98

Charcoal, adsorption by, 16

Chemical action on blood vessels, 147

- affinity, 14

- combination, 36, 44

- - nature of, 15

- energy, II

- formulæ, 44

- inertness of colloids, $35^{\circ}$

- messengers, 136

- potential, 14, 90

- - raised by adsorption, 70

- senses, I07

- stimulation of glands, 82

Chlorophyll, 5I, 55

- absorption spectrum of, 186

Chloroplasts, 54

Ciliary muscle, 115,213

Circulation in frog's web, 22 I

- model of, 2 I 8

- of blood, 132

Clotting of blood, I4I

Cochlea, 108

- preparations of, 212

Cohesion, 5

Cold, I04, 106

Colloidal complexes, 36

- gold, 176

- ion, 33

- state, 30, 32

Colour perception, 117

Combustion, I I

- in air, 189

- in animals, 11

- mechanism of, 72

- of sugars, 72

Concentration of reagents, 68

Conditioned reflexes, 127

Cones of retina, I 16

Conjugation, 152

Consciousness, 103, 105, I20, I 24

Constituents of diet, 65 
Continuity of germ plasm, I 53

Continuous phase, 30

Contractile vacuole, I7 I

Contraction of muscle, 93, 204

- - by stretching, 80

Contractures, 100

Co-ordination of eye and hand, II9

Cornea, 115

Coronary arteries, 222

Corpus luteum, $\mathrm{I} 56$

Corpuscles of blood, 135, 223

Cortex inhibited, $\mathbf{I} 29$

Cortical points, stimulation of, 129

Cream, 30

Cross-fertilisation, 154

Curare, 199

Current of injury, 210

DARK-GRÓUND illumination, 4, 166

Deamination, 76

Deficiency diseases, $6 \mathrm{I}$

Degeneration of nerves, 121

Degradation of energy, 12

Denitrifying organisms, 58

1)epression of freezing point, 22

Depressor reflex, 146

Desensitisation, I 4 I

Development of the frog, 226

Dextrin, 70

Diabetes, 136

Diagrams, viii.

Diaphragm, 88

Diet, constituents of, 65

Digestion, 66

Direct adaptation, 158

Dispersed phase, 30

Dissipation of energy, 13

Dissociation of oxy-hæmoglobin, 86

- tension, 87

Distance receptors, 107, 120

Distribution of water between phases, 3 !

Dividing nuclei, 226

Dogmatic presentation, vi.

Dominant, 156

Dorsal root ganglia, 124

Drugs, action of, on the heart, 225

Drum of ear, 108

EARTHWORM, nervous system of, 123 Economical use of energy, I3

Effectors, 121
Efferent fibres, 124

Egg-cells, 153

Electric charge on colloids, 33

- waves, 113

Electrical adsorption, 36, 38, 179

- change in nerve, 130,210

- changes in secretion, 83, 197

- charge on colloids, 177

- resistance of living cells, 28

- stimulation of amœba, 165

Electrodes, 24

- for heart, 207

Electrolysis, 24

Electrolytes, 25

- action of, on colloids, 35

- precipitation of colloids by, 178

Electrolytic colloids, 33

- conductivity, 173, 174

- dissociation, 23

Electrolytically dissociated colloids, 33,34

Electrons, 6

Emulsions, 30

Emulsoids, 30, 177

Energetics, Io

- laws of, 10, II

Energy, 10

- of muscular work, 93

- requirements of man, 63

Enterokinase, 82, 193

Entropy, 13

Enzymes, 66, 189

Equation of state, 22

Equilibrium and death, 14

- in ester and water, 68

- in photo-chemical reaction, 117

- under enzyme action, 67

Equimolecular solutions, 19

Erepsin, 75

Esters, 64, 65

- hydrolysis of, 67

Ethyl-glucoside, synthesis of, by emulsin, 191

Evolution of nervous system, 122

Examinations, viii.

Excitation, 125

Excitatory nerves, 79, 149

Exhaustion, 126

Experiments, value of, vi.

Expired air, analysis of, 185

Explanation, meaning of, 7

Extent of surface, 3I

Extero-ceptors, 119

Eye, 112

- anatomy of, 213 
FARADAY phenomenon, I 76

Faraday's platinum experiments, 69

Fat from carbohydrates, 72

- in intestinal epithelium, 195

Fatigue in nerve, 130

- of muscle, 94, I 26

- of nerve centres, 126

Fats, 64, 65, 73

Fat-soluble A-factor, $6 \mathrm{I}$

Feeding on amino-acids, 46

Female gamete, 153

Fenton's reaction, 90

Ferric hydroxide, 178

Fertilisation, I 53

- in plant, 227

Fever, IoI

Fibrillæ of muscie, 96

Fibrin, 14 I

- preparation, 223

Filtration in glomeruli, 138

Final common path, I23, I24

First law of energetics, I I

Flagella, 56

"Flesh-formers," 64

Flow of water by osmosis, 197

Flower, structure of, 227

Food in hot weather, Ior

- of muscle, 95

-- use of, Io, 40, $4 \mathrm{I}$

Form, perception of, I 19

Formaldehyde, 43, 54

Free energy, I 2

Freezing point, 172

- - of solutions, 22

Frog heart perfusion, 187

Fructose, 44, 72

Function of viscosity of blood, I 45

GALACTOSE, 72

Galvanometer, 173, 197

Gamboge, 4

Gametes, I 52

Ganglion, I23, 131

Gas analysis, I 83

- phase, 9

Gastric juice, 7 I

General principles, value of, $v$.

Germ plasm, I 53

Gizzard, 66

Glands, 78

Glomeruli of kidney, 138

Glycerol, 64, 65

Glycine, 45

Glycogen, 71

Gradation of contraction, 97
Granules in gland cells, 81, 196

Gravity, response of plants to, 137

Green plant, 50

- action of, on carbon dioxide, 183

Growth, 40, I 5 I

Guaiacum, 9I

- reaction, 203

Gum solutions for intravenous injection, 140

HABIT, I 29

Hæmatocrite, I 70

Hæmoglobin, 55,85

- carriage of oxygen by, 200

Hæmolysis, 17, I70

Hæmorrhage, arrest of, 142

Hairs and touch, Io6

Harmonics, II 2

Harvey, I 34

Hearing, IO7

Heart, I 32, I 34

- of sheep, 222

Heat, 104, 106

- and cold spots, 2 I I

- centre, IOI

- energy, I I, 12

- relaxation of muscle by, 97

"Heat-givers," 64

Helmholtz double layer, 34,83

Heredity, 156

Heterogeneous catalysis, 69

- systems, 8

Hexoses, 42

Hibernation, IOI

Histamine, action of, on capillaries, 147

Histological preparations, 193

- staining, 37

Hormones, 84, I 36

Hunger, 80

Hydration of ions, 25

Hydrogen electrode, 27

- test for, 181

Hydrogen-ion, 25

- concentration, 27

-- - and breathing, 89

- regulation of blood, 224

Hydrolysis by enzymes, 190

Hydrolytic dissociation, 38

IMAGE on the retina, 164,213

Imbibition, 3 I, 97

"Immortality" of protozoa, I 52

Immunity, I4I 
Increase of chemical potential by adsorption, 70

Indicators, 27, 175

Induction coil, 205

Inheritance of acquired characters, 157

Inhibiting substances in plants, 137

Inhibition, 124, 126, 127, 128, 129

- from cortex, 130

- of inhibition, 129

- of reflexes, 217

- of the heart, 217

Inhibitory nerves, 79, 149

- - to heart, 149

Insolubility, 33

- of products, 72

Instability of cortical points, 129

Integration in organisms, 103

Intensity factor, 14

Interfaces within cells, 17

Intermediate compounds, 69

Internal phase, 30

- pressure of liquids, 5,8

- secretions, 84, I 36

Intero-ceptors, I 9

Interstitial cells, 136, I 55

Invertase, 72, 193

Involuntary muscle, contraction of, 196

- structure of, 195

Ions, 24

- velocity of, 25

Isotonic solutions, 22

I ris, II 5

Iron in cells, 54

- in chloroplasts, 54

- in hæmoglobin, 86

Isomers, optical, 48

KEITH-FLACK node, 150

\section{Ketone, 44}

Ketonic acids, 76

Ketoses, 44

Kidneys, I 36, 137

Kinetic energy, I I

- theory, 4

\section{LABYRINTH, I I 8}

Lactic acid, 73

- - in blood, 143

- - in fatigue, 126

- - oxidation of, by Fenton's reaction, 203

_ - produced by alkalinity, I 44

- - production in muscle, 95
Lactone, 44

Large intestine, 77

Law of progress, 216

- of the heart, 148

- of the intestine, 80

Laws of energetics, I0, II

Lecithin, 65

Length of fibres and energy of muscle, 96, 149, 206

Lens, I I 4,163

- of eye, II 4

Leucocytes, 135, I65, 223

Life as transfer of energy, 90

- characteristics of, I, IO, I4

Light, 5 I, 104, 107, I1 3

Linseed, 72

Lipase, 74,190

Lipines, 65,90

Liver, 7 I, 74, 75, 77

- blood supply of, 135

Local sign, 106

Lovén reflex, 146

Lowering of surface energy, 15

Lungs, 87,88

Lymph, I 40

Lymphatic glands, I 4 I

Lymphatics, 74, 140

Lysine in proteins, 143

MAGNESIUM in chlorophyll, 55

Maintenance, 40, I 5 I

- of temperature, IOI

Male gamete, I 53

Maltase, $7 \mathbf{I}$

Maltose, 71,72

Mammary glands, 155

Manometer, 2 I

Mass action, 68

Measurement of microscopic objects, 169

Mechanism of combustion, 72

- of secretion, 82

Memory, 129

Mendelism, I 56

Mental development, 120

Metabolism, 63

Metals in peroxidase, $9 \mathrm{I}$

Methyl acetate, hydrolysis of, 189

Micro-organisms, 56

Microscope, 163

- invention of, I 34

Microtome, 194

Milk, I 55

- sugar, 72 
Mitochondria, 39

Model of catalysis, 192

- of circulation, 218

Mol, 19

Molar concentration of proteins, 143

- solutions, 19

Molecular concentration, 19

Molecules, 4

Motor areas, 129

- centre, 120

Movements, 93

- of alimentary canal, 78

Muscle system, nature of, 95

Muscles, 79

Muscular sense, I 19

- work and loss of structure, 40, 62

Mutual aid, 58, 159

- precipitation of colloids, 179

Myograph, 205

NAtural selection, 158

Nature of muscle system, 95

- of nerve impulse, 130, I31

Negative after-image, 116

- temperature coefficient of muscle, 96

Nerve, electrical change in, 210

- impulse, 130

- structure of, 209

- unexcited by sound or light, 2 Io

Nerve-cell, I 2 I

Nerve-muscle preparation, 204

Nerves, 104

Neurone, I 2 I

- structure of, 216

Neutral red, 27

Neutrality of blood, 142

- regulation by kidney, I44

Newt's gastric glands, 197

Nitrates as plant food, 57

- formed in soil, I86

Nitrogen, source of, 45

- test for, I8I

Nitrogen-cycle, 56,77

Nitrogen-fixing organisms, 58

Nocuous stimuli, ro6

Non-polarisable electrodes, 197

Nuclear division, 152

Nucleus, 3, 39, 42, 12 I

OCEAN, composition of, 60

Oedema, 140

Oesophagus, 7 I
Oils, 65

Olein, 65

Olfactory cells, 2 I I

Olive oil, 65

Optical activity, 48

- isomers, 48,49

- sensitizer, 52

Organ of Corti, 108, 109

Organic compounds, 4I

Origin of heart beat, I 50

Osmometer, I7 I

Osmosis, 17

Osmotic pressure, 21, 169

Ova, 153, 226

Ovary, I 53

Oxidase, 9 I

- of potato, 203

Oxidation, mechanism of, 89

Oxidising enzymes, 76

Oxygen consumption and work, 100

- test for, I8I

Oxy-hæmoglobin, 87

PACE-MAKER of the heart, 225

Pain, I04, 106

Pancreas, granules in, 196

Pancreatic juice, 7 I , 74, 75

Paralysis, 80

Parthenogenesis, 153

Partial pressure, 85

Penis, I 54

Pentoses, 42

Pepsin, 75, I93

Peptide linkage, 47

Peptones, 75

Perception of gravity, 117

Perfusion fluids, 59, 142

- of frog heart, 223

Peripheral resistance in arterioles, 145

Peristaltic contraction, 79

Permeability change in secreting cells 82,84

- of blood vessels, I37, I40

- of cell membranes, 9 , I0, 16

- of cells, 168

- of membranes to solids, IO

Peroxidase, 91

- nature of, 91

- of horse-radish, 203

Peroxides, 55, 90, 203

Phagocytosis, 135

Phase, 8

- difference in compound sounds, 112

Phloroglucin, 2 I 2 
Phosphates, excretion of, 144

Phosphorus food, 58

- test for, $18 \mathbf{r}$

Photo-assimilation, 54

Photo-chemical reactions, 113,116

Physical properties and chemical constitution, 35

Physiology, I, 7

Pigment of eye, 116

Pilocarpine, 82

Pitch of notes, 107

Placenta, I 54

Plasma, I 35, I 36

Plasma-membrane, 9,15

Plasmolysis, 20, 17 I

Platelets, 223

Poison gases, 86

Polarimeter, 49 , I8I

Polarised light, 49, I 13

Pole-finding paper, 177

Pollen grains, 153

Pollen-tube, 227

Polypeptides, 47

Position in space, 104

- receptors, II7

Posture phenomena, 99

Potassium as radio-active element, 60

- function of, 142

Potential energy, I I

Practical value of science, 2

Precipitation by electrolytes, 178

- of colloids by electrolytes, 35

Proprio-ceptors, I 19

- and tonus, 100

Proteins, 47, 75

- of the plasma, I4I

Protoplasm, 2, 7

- chemical composition of, $4 \mathrm{I}$

Protoplasmic movements, 166

Protozoa, 56

Pseudopodia, 3, 7, 9

Psychology, I

Pupil of eye, II 5

Putrefaction, 56

Pyrrol, 55

Pyruvic aldehyde, 76

QUALITY in sensation, 106

- of musical notes, I08, I I I

RADIANT energy, I I

Radio activity of potassium, I 42
Rate of conduction of nerve impulse, I 30

- reaction between colloids, 179

Rates of chemical reactions, 67

- of reactions, I 89

Reabsorption in kidney, 139

Receptors, 103

Recessive, 156

Reciprocal innervation, I25, 146

Red beet root, experiments with, 168

Red corpuscles, 85

- - destruction of, 135

- - formation of, 135

Red marrow, I 35

Reduction, 92

- by milk, 203

Reflex action, $80,-103,-120,-124,209$, $=16$

$-\operatorname{arc}$, I 2 I

Refractory phase of nerve, 130

- period, 98, 208

Regulation of blood supply, I45

- of heart beat, 148

- of reaction of blood, 143 .

- of temperature, IOI

Rejuvenescence, 152

Removal of products of reactions, 72

Reproduction, I 5 I

Resistance of blood corpuscles, 175

Resonance, 52, I10

- of membrane, 212

Respiration, 84

Respiratory quotient, 95

Restitution phase in muscle, IOI

Re-suspension of colloids, 36

Reticular membrane, I Io

Retina, I14, I 16

- structure of, 214

Ringer's solution, 224

Ring form of amino-acids, 47

Rods of retina, 116

Roman farming, 58

Root nodules, 58,187

- pressure, 82

"Rust" in wheat, 157

SALIVA, 66

- and thirst, 84

Salivary glands, nerves to, $8 \mathbf{I}$

- reflex, 128

Salts in food, 59

- of the blood, I42, 223

- required for growth, 60

Saponin, 15 
Saponin, action of, on enzymes, 70

- bubble, 168

Sarcoplasm, 96

- and tonus, 100

Sciatic nerve, position of, 199

Scurvy, 6r

Sea anemone, nervous system of, 123

- water, 60

Second law of energetics, 12

Secondary sexual characters, I 55

Secretin, 82, 137

Secretion, 78, $8 \mathrm{I}$

- disappearance of granules in, 196

Segregation of gametes, I 57

Semi-circular canals, I 18

- - of skate, 2 I 4

Semi-permeable membranes, 19

Sense-organs, 103

Serum, 142

Sex, 152

Shivering, Ior

Shock after wounds, I 47

Silver chloride, action of light on, I I6

Sine curve, I I 2

Size, perception of, I 19

Skin receptors, 106, 210

Small intestine, 7 1

Smell, 104, 107

Smooth muscle, properties of, 79

Soap bubbles, 167

- film, 6, Io

- solution, 167

Sodium bicarbonate in blood, 143

Solids, permeability to, 10

Solution of gases, 86

Sound, $104, \mathrm{IO}_{7}$

Sources of carbon, $4 \mathrm{I}$

Specialised receptors, 107

Specific sense energies, 105

Spectroscope, 52

Speech and writing, I 20

Spermatozoa, I 53, 226

Spinal cord, 120

frog, I03, 124, 209, 216

Splanchnic nerve, 80

Sponges, 122

Spores, 56, I $5 \mathrm{I}$

Spring for muscle model, 204

Staining, 179

- of histological preparations, 37

"Staircase," 98, 207

Stannius' ligature, 207

Starch, 53

- formation of, 186

Statocyst of Cyclas, 214
Statocysts, II7

Stearin, 65

Sterilisation, 57

Stimulus, 94

Stomach, 7 I

- of frog, contractions of, 196

Storage of fat, 74

Strength of acids, 175

- of acids and bases, 26

Structure of muscle, 96

Struggle for existence, I 3, 56, 159

Sugar, test for, 169

Sugars, 4I

Sulphur food, $5^{8}$

- test for, I8I

Supra-renals, I 3 I, I 36

Surface action by enzymes, 192

- energy, 14

- tension, 6, 8, 9, 167

- - and dispersion, 177

- - in muscle, 96

- - of colloids, 32

Suspensoids, 30

Sweat glands, 84

Sweating, IoI

Swelling of colloids by acid, 97

- of gelatin, 177

Symbiosis, 58

Sympathetic system, I3I, I 46

- of frog, 215

Synapse, I2I, I 22

Synaptic system, I 23

Synthesis and hydrolysis by catalysts 68

- by enzymes, I9I

TASTE, IO4, IO7

Taste-buds, 2 I I

Tectorial membrane, IIo

Teeth, use of, 66

Telephone exchange, 127

Temperature, effect of, on muscle, 206, 225

- regulation, Ior

Temporary association, 127,128

Tension of active muscle, 94

- of gas, 85

Testis, 153

Tetanic contraction, 98

Tetanus of muscle, 206

Thermodynamics, 10

Thirst, 84

Thoracic duct, $\mathbf{I} 40$

Thought and memory, 124 
Three constituents of diet, 65

Thyroid, I 36

Titration, 28

Tonic contraction, 97

Touch, 104, 106

Toxins, 57

Tracheæ of insect, 85,200

Tradescantia hair, I66

Transmission of heart beat, 150

Transport of materials, I 32

Trigger-action, 97, I05

Trophic influence, 122

Trypsin, 75, 193

Tryptophane, 46

Tubules of kidney, 138

Turgor, 22, I7 I

Tyndall phenomenon, 176

ULTRA-MICROSCOPE, 4, 32 .

Unconditioned reflexes, I 27

Unsaturated carbon atom, 65

- fats, 90

Urea, 50, 77, I 37

- test for, 182

Urease, 77,182

Ureter, I 38

Urethane for frog, 22 I

Uric acid, 137

Urinary bladder, 99

Utricle and saccule, I 8

VACUOLE, 20

Vagus nerve, 79, I 49

- - of frog, 217

Value of experimental work, vi.

- of general principles, $v$.

Van der Waals' equation, 22

Vaso-constriction, 220

Vaso-constrictor nerves, 146

Vaso-dilatation, 220
Vaso-dilator nerves, 146

Vaso-motor centres, 146

- reflexes, 146

- stimulation, 224

Vapour pressure, 23, 173

Variation, I 52, I 57

Veins, I 34

Velocity of ions, 25

- of reactions, 68

Vena cava, 134

Venous blood, 87

Ventricle, I 32

Visceral nervous system, I3I

- sensory nerves, 131

Viscosity, I 44

- of blood, I44, 22 I

Visual purple, II 4, I I 7, 2 I 2

Vitamines, 61

Voluntary contraction, 98

- muscle, structure of, 195, 206

WAR, 159

Warm stage for microscope, 166

Warming sinus, 225

Waste products, 137

Water culture, 183

- effect of, on equilibrium position, 68

- loss of, from body, 84

Water-soluble B-factor, 6 I

Wheatstone bridge, 175

Whiskers of cat, 106

Wireless telegraph, II 3

Work, Io

"Work-collector," 205

X-RAYS, II 3

- use of, in investigating movements of intestine, 78 


\section{SOURCES OF SUPPLIES}

Arc Lamp.-G. Cussons LTD., The Technical Works, Manchester.

Articulated Bones and Models.-Messrs Deyrolle, Paris.

Balance.-F. E. BECKer \& Co., Nivoc House, Hatton Wall, E.C.I.

L. Oertling, Turnmill St., E.C.

Castor Oil Seeds.-James CarTer, Seedsmen, High Holborn, W.C.I.

Centrifuges.-Chas. Hearson \& Co., 235 Regent St., W.I.

Chemical Apparatus.-BAIRD \& TATLOCK, 14 Cross St., Hatton Garden, E.C.I.

Chemicals.-Hopkin \& Williams, 16 Cross St., Hatton Garden, E.C.I.

Drugs.-W. Martindale, io New Cavendish St., W.I.

Electrical Apparatus in General.-R. W. PAUL, Electrical Laboratory, Fortis

Green Road, Finchley, N.2

W. G. PyE \& Co., Granta Works, Cambridge.

Galvanometers.-The Instrument Co., Cambridge.

Glass Blowers.-JOHN ORME \& Co., I 48 High Holborn, W.C.I

Histological Preparations.-WATson \& Sons, 31 3 High Holborn, W.C.I.

Lenses.-PyE \& Co., Granta Works, Cambridge.

Microscopes and Fittings.-SWIFT \& SON, 8I Tottenham Court Road, W.I.

BAUSCH \& LOMB, 37 Hatton Garden, E.C.I.

Spencer Co., Agents-H. F. Angus \& Co., 83

Wigmore St., W.I.

Microtomes.-The InSTRUment Co., Cambridge.

Osmometer.-C. F. Palmer, 55 Effra Road, Brixton, S.W.2.

Physiological Apparatus (Drums, Myographs, Heart Apparatus, Coils, etc.).-

C. F. PAlmer, 55 Effra Road, Brixton, S.W.2.

Plasticine.-HARBUTT, 56 Ludgate Hill, E.C.4.

Plate Glass Shelves.-MAPLE \& Co., Tottenham Court Road, W.I.

Platinum.-Johnson \& MatThey, Hatton Garden, E.C.I.

Polarimeter.-AdAm Hilger, LTD., 75A Camden Road, N.W.I.

Skate and Marine Animals.-Marine Biological Laboratory, Citadel Hill, Plymouth.

Soy Beans.-Messrs Shearns, 23I Tottenham Court Road, W.I.

Surgical Instruments.-HAwKSLEY \& SON, 357 Oxford St., W.I.

J. WeISS \& Son, 287 Oxford St., W.I.

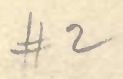





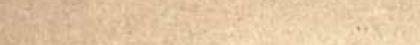

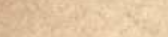

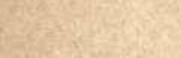

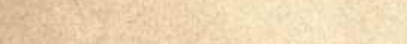

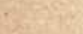

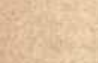

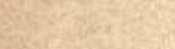

zanivit 

THIS BOOK IS DUE ON THE LAST DATE STAMPED BELOW

\section{AN INITIAL FINE OF 25 CENTS} WILL BE ASSESSED FOR FAILURE TO RETURN THIS BOOK ON THE DATE DUE. THE PENALTY WILL INCREASE TO 50 CENTS ON THE FOURTH DAY AND TO \$1.00 ON THE SEVENTH DAY OVERDUE. DO

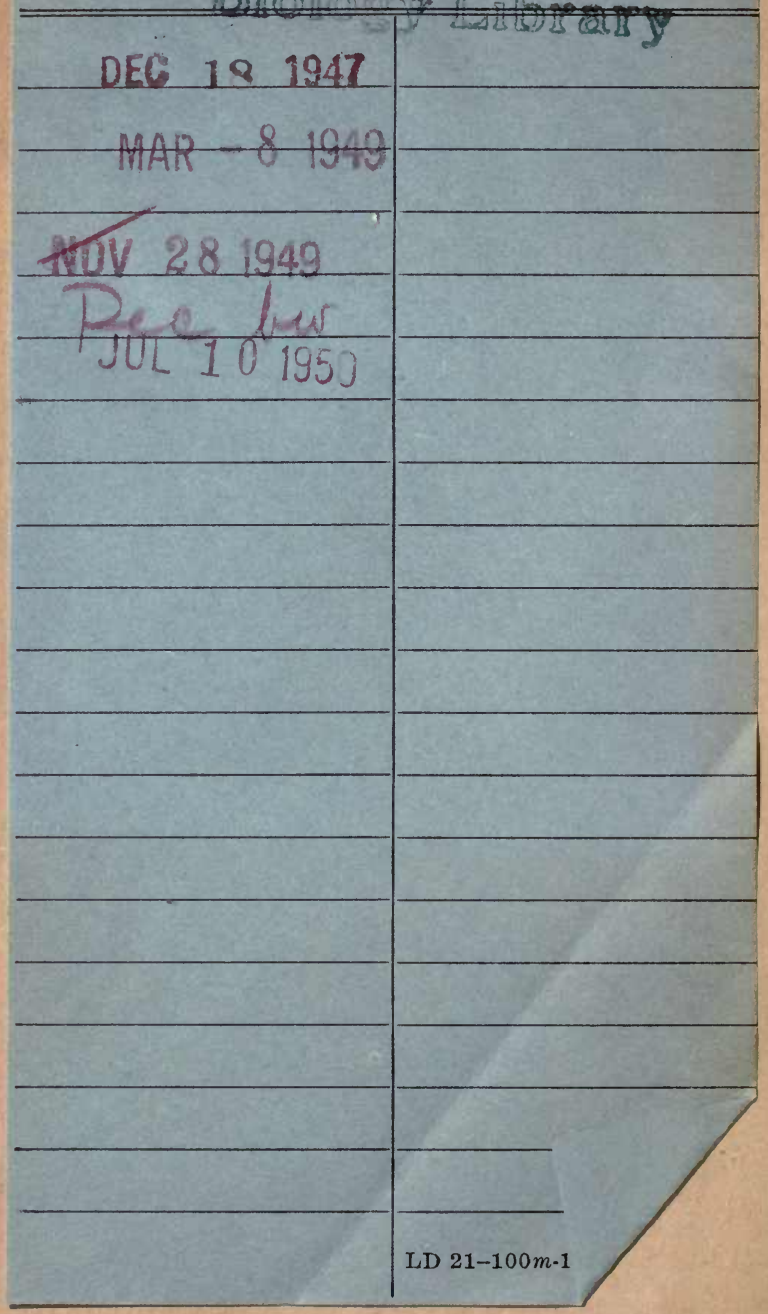




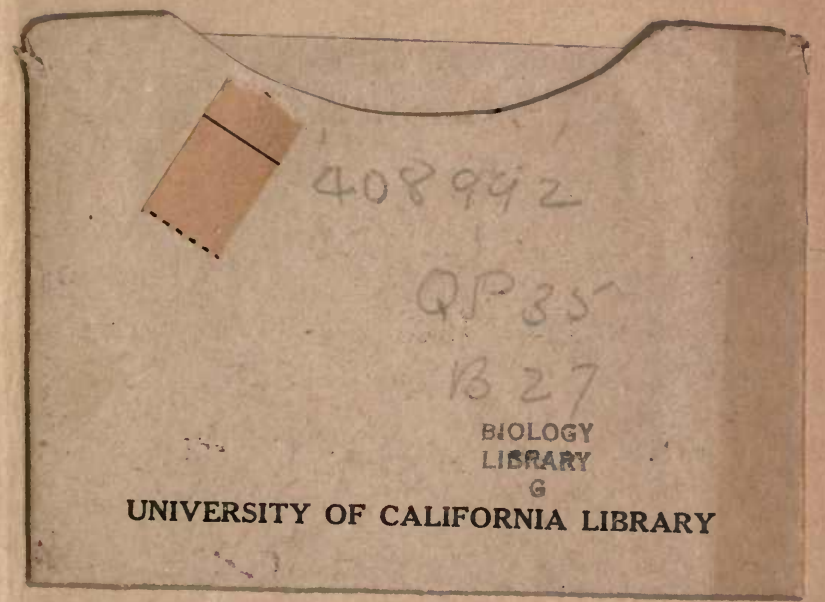


